\title{
DEVELOPMENT OF A HIGH PRECISION QUANTUM DOT SYNTHESIS METHOD UTILIZING A MICROFLUIDIC REACTOR AND IN-LINE FLUORESCENCE FLOW CELL
}

\author{
A Thesis \\ Presented to \\ the Faculty of California Polytechnic State University, \\ San Luis Obispo
}

\author{
In Partial Fulfilment \\ of the Requirements for the Degree \\ Master of Science in Engineering
}

By

William Henry Lafferty

November 2014 
(C) 2014

William Henry Lafferty

ALL RIGHTS RESERVED 
TITLE:

AUTHOR:

DATE SUBMITTED:

COMMITTEE CHAIR:

COMMITTEE MEMBER:

COMMITTEE MEMBER:
Development of a High Precision Quantum Dot

Synthesis Method Utilizing a Microfluidic Reactor

and In-Line Fluorescence Flow Cell

William Henry Lafferty

September 2014

Richard Savage, $\mathrm{PhD}$

Director of Graduate Education

Hans Mayer, MS

Lecturer of Mechanical Engineering

Lily Laiho, $\mathrm{PhD}$

Professor of Biomedical Engineering 


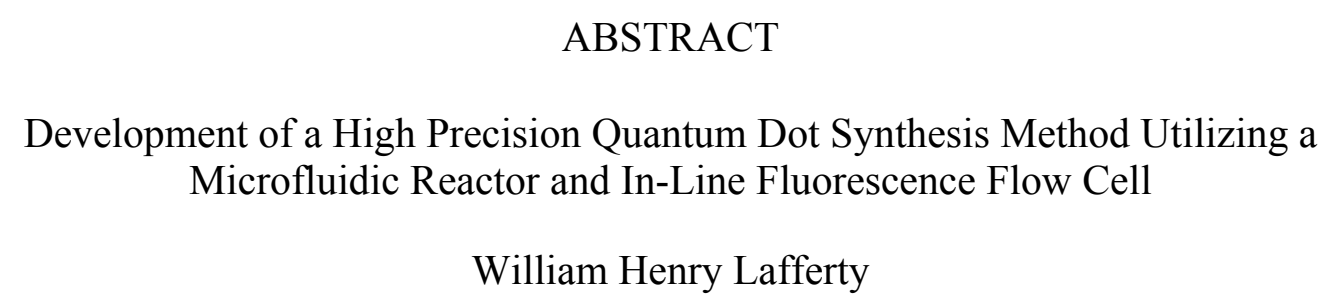

Quantum dots show great potential for use as spectral converters in solar cells, lighting applications, and biological imaging. These applications require precise control of quantum dot size to maximize performance. The quality, size, and fluorescence of quantum dots depend on parameters that are difficult to control using traditional batch synthesis processes. An alternative, high precision process was developed for the synthesis of cadmium-selenide quantum dots using a microfluidic reactor and fluorescence flow cell. The process required creating separate cadmium and selenium precursors that were then mixed in a nitrogen environment at $17^{\circ} \mathrm{C}$. Using an NE-300 ${ }^{\circledR}$ syringe pump, the solution was pumped through a microfluidic reactor submerged in a $240^{\circ} \mathrm{C}$ oil bath. The reactor then fed through a water quench bath at $25^{\circ} \mathrm{C}$ to terminate the nucleation and growth reaction. The fluorescence profiles of the quantum dot solutions were then characterized with an in-line fluorescence flow cell used in conjunction with an Ocean Optics ${ }^{\circledR}$ USB $40000^{\circledR}$ spectrometer and a ThorLabs ${ }^{\circledR}$ LED UV light source. Flow rates through the reactor were varied from $0.05 \mathrm{ml} / \mathrm{min}$ to $2 \mathrm{ml} / \mathrm{min}$. A central peak wavelength was registered in the fluorescence profiles for each flow rate. Monodisperse Cd-Se quantum dot solutions were synthesized across a broad spectrum of wavelengths ranging from $490 \mathrm{~nm}$ to $620 \mathrm{~nm}$. An empirical relationship between flow rate and center wavelength was determined.

Keywords: Quantum Dots, Microfluidics, Fluorescence, Flow Cell, Cadmium-Selenide 


\section{ACKNOWLEDGMENTS}

I would like to thank the following people for making this project possible:

- Dr. Richard Savage (Materials Engineering) for his guidance and support

- Dr. David Clague (Biomedical Engineering) for assistance with COMSOL modeling

- Peter Gonsalves for his previous work in microfluidics

- Laura Sparks for her previous work on conventional quantum dot synthesis

- Aaron Lichtner for his research on quantum dot synthesis

- Johnny Hoadley for his contributions in segmented flow analysis and fluorescence flow cell analysis techniques.

- Ross Gregoriev for assistance in quantum dot imaging and countless design discussions.

- Logan Grimes for assistance creating a quantum dot precipitation process

- Cal Poly Microsystems Technology Club and related facilities 


\section{TABLE OF CONTENTS}

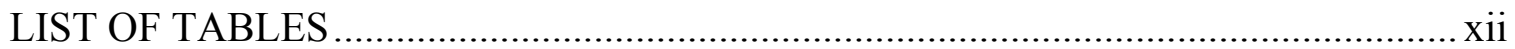

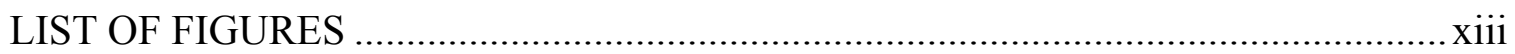

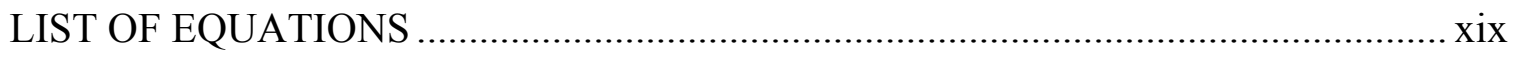

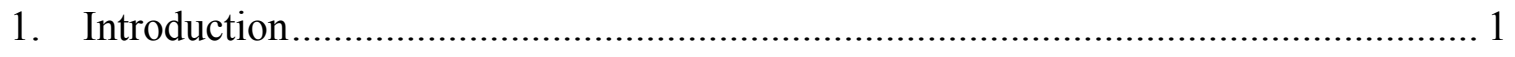

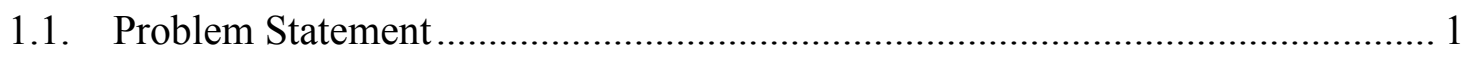

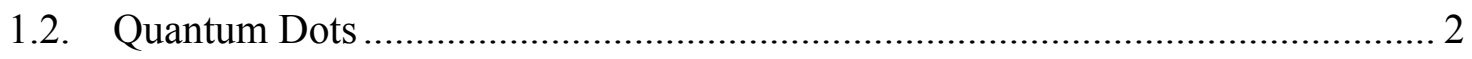

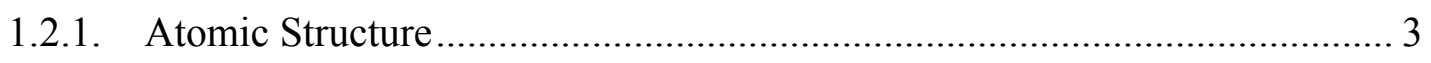

1.2.2. Quantum Confinement......................................................................... 5

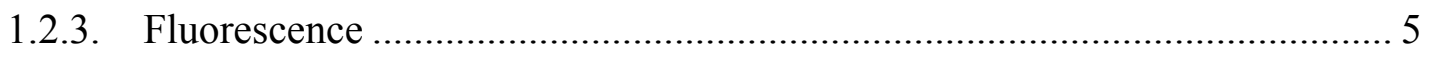

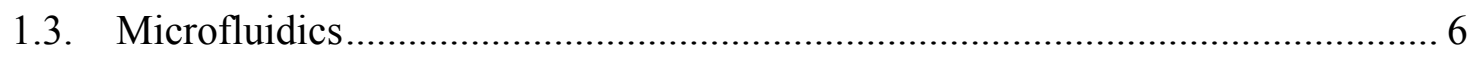

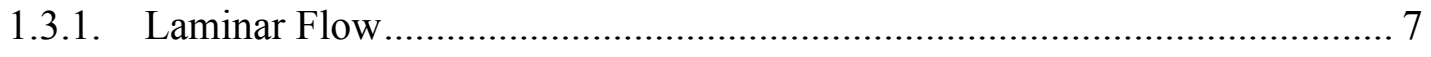

1.3.2. Fluid Resistance and Pressure.................................................................... 8

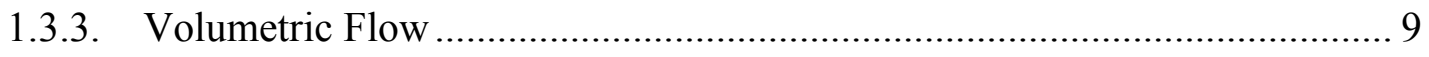

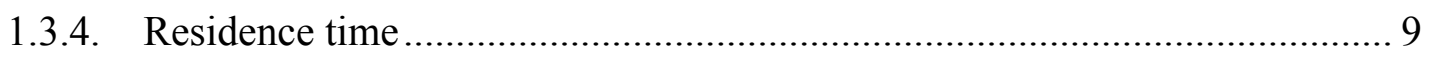

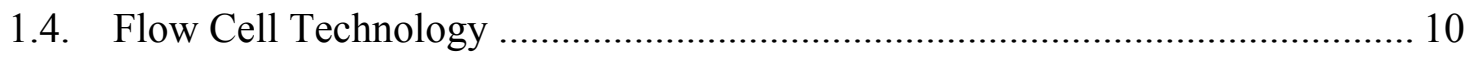

1.4.1. Flow Injection Analysis (FIA) .............................................................. 10

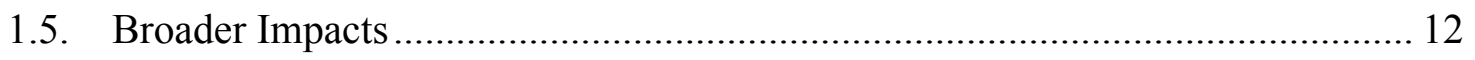

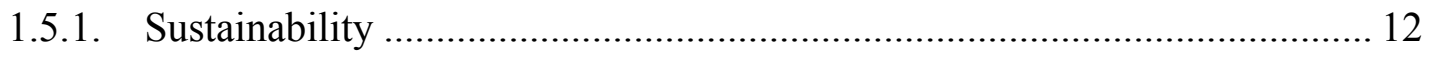

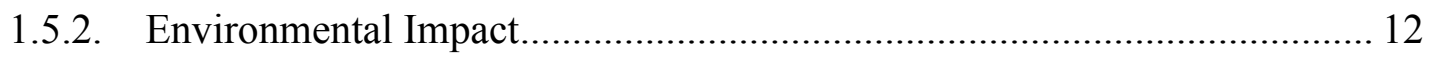

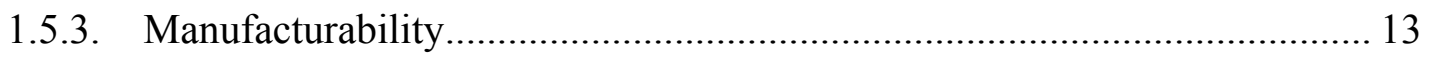

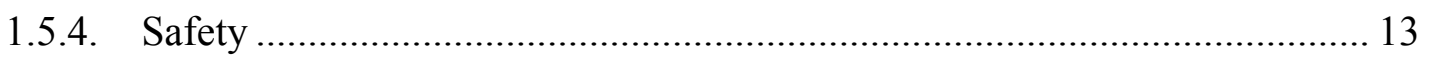


2. Background on Nucleation and Growth Theory ……….................................... 15

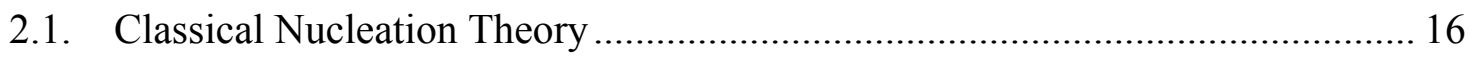

2.1.1. Homogeneous Nucleation ................................................................... 16

2.2. Nucleation and Growth Thermodynamics ................................................... 17

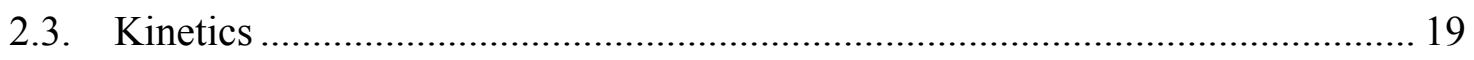

2.3.1. Precursor Solution Kinetics …………………................................... 20

2.3.2. Nucleation and Growth Kinetics............................................................... 20

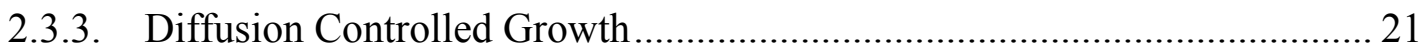

2.3.4. Reaction Controlled Growth ................................................................. 22

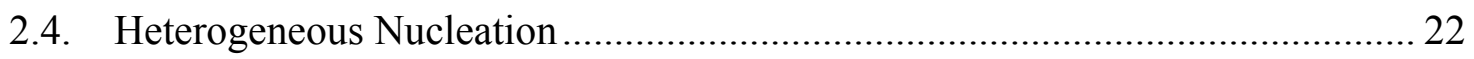

3. Proof of concept (P.O.C) Microfluidic reactor system ............................................. 23

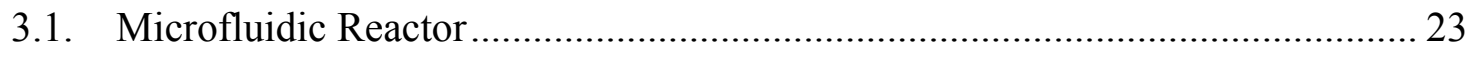

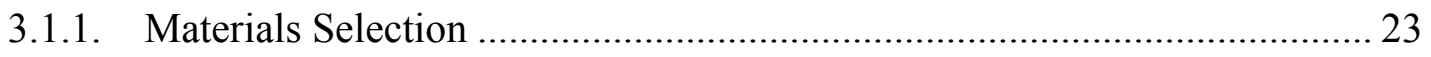

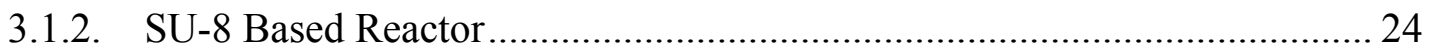

3.1.3. Patterned Silicon Wafer and Pyrex Wafer Reactor .................................... 25

3.1.4. Prefabricated Tubing Reactor ………………........................................... 25

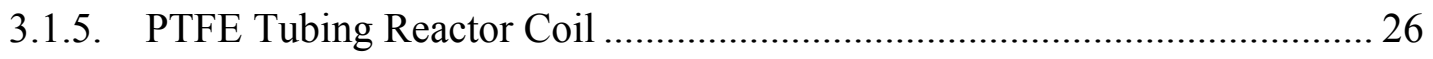

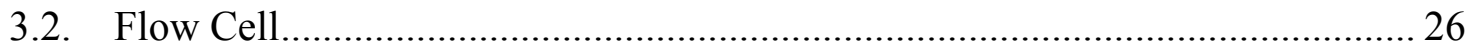

3.2.1. Flow Cell Light Source............................................................................. 27

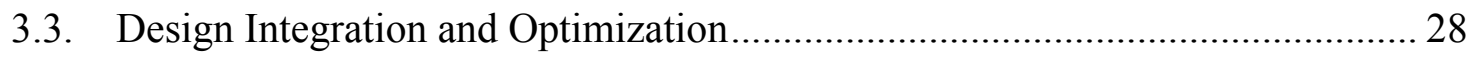

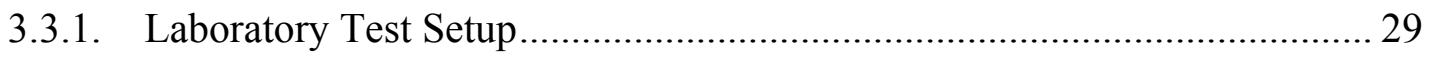

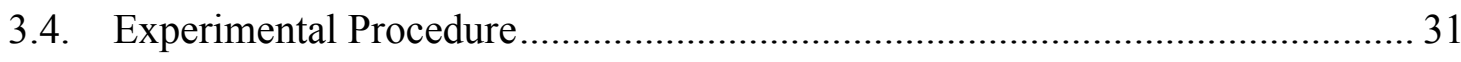

3.4.1. Microfluidic Synthesis Procedure ................................................................. 31 


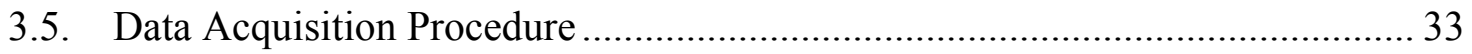

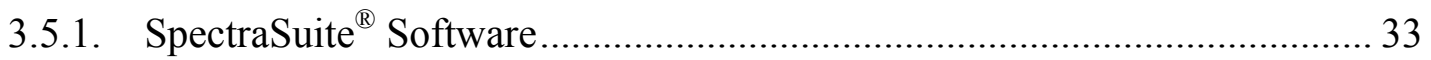

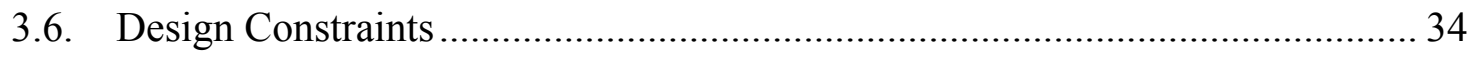

3.7. Proof of Concept Reactor Results..................................................................... 35

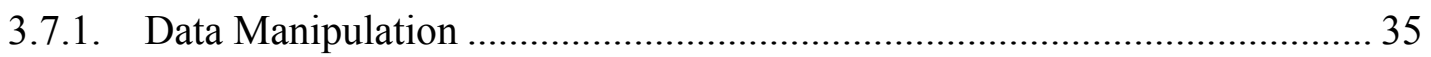

3.7.2. Moving-Median Smoothing (P.O.C Reactor)............................................... 35

3.7.3. Relationship of Flow Rate to Peak Center Wavelength .............................. 37

3.7.4. Analysis of Particle Diameter ...................................................................... 39

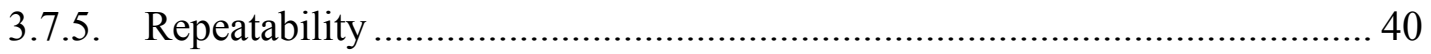

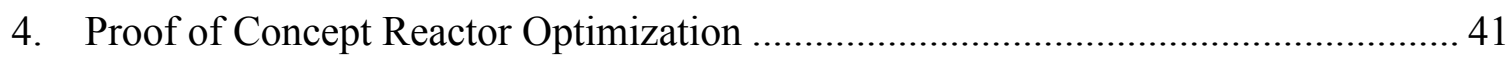

4.1. Light Source and Light Source Fixture Modification......................................... 41

4.2. Modified Precursor Cooling Method ................................................................... 42

4.2.1. Ethylene Glycol Chiller Unit Construction ................................................ 42

4.2.2. Ethylene Glycol Chiller Testing and Results................................................ 44

4.2.3. Ethylene Glycol Chiller Discussion............................................................ 45

4.3. Optimized Experimental Procedure ................................................................. 46

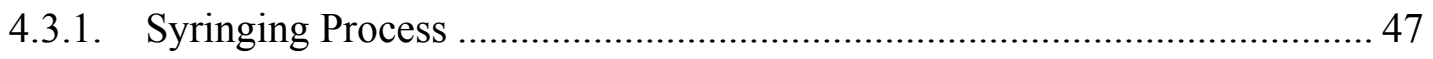

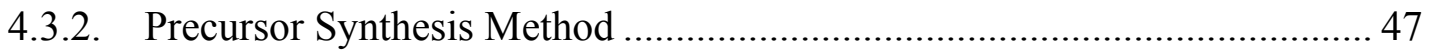

4.3.3. Precursor Solution Mixing Procedure ............................................................ 49

4.3.4. Reactor Characterization Procedure ............................................................ 50

4.3.5. Optimized SpectraSuite ${ }^{\circledR}$ Data Acquisition Procedure................................. 51

4.3.6. Data Manipulation with MATLAB ……………………………………..... 53

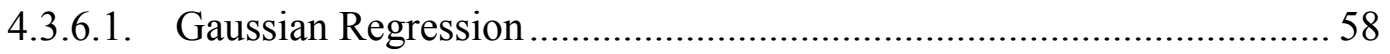


4.4. 316 Stainless Steel Reactor Fabrication

4.4.1. Heat Treatment 60

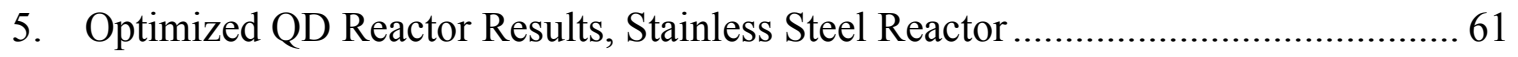

5.1. Relationship of Flow Rate to Peak Center Wavelength ................................. 63

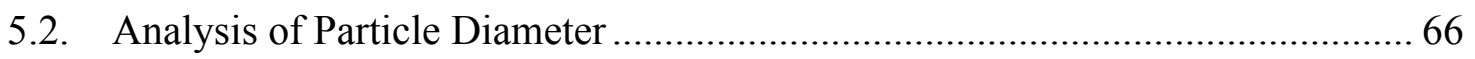

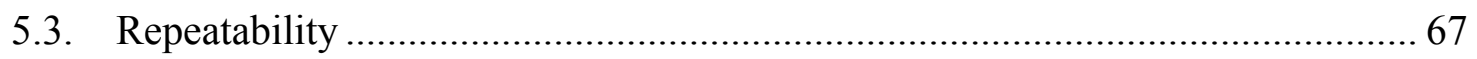

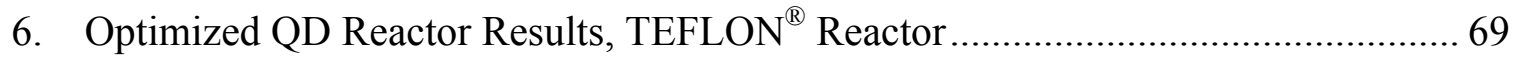

6.1. Relationship of Flow Rate to Peak Center Wavelength ................................. 72

6.2. Analysis of Particle Diameter ........................................................... 75

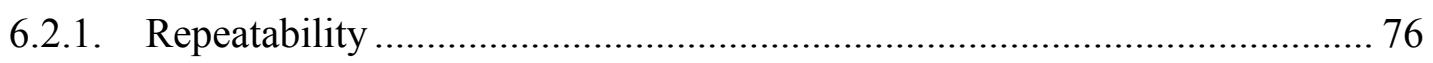

6.3. Comparison of Repeatability Between the Optimized TEFLON ${ }^{\circledR}$

Reactor and Optimized 316 Stainless Steel Reactor ......................................... 78

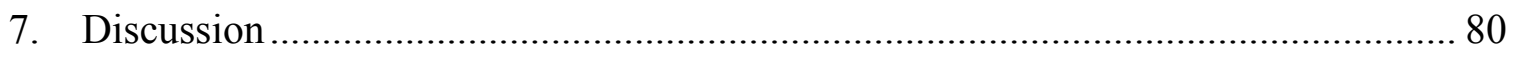

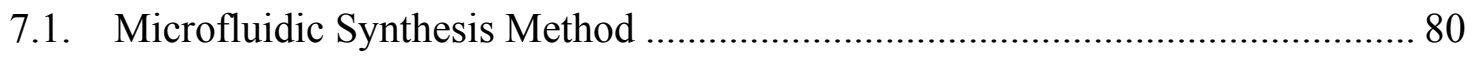

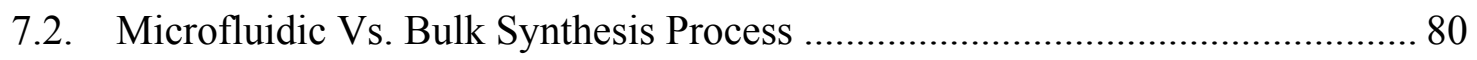

7.3. Benefits of Flow Cell Analysis .................................................................... 82

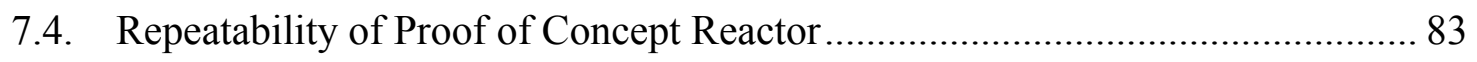

7.5. Repeatability of Optimized Reactors .................................................. 84

7.6. Investigation of Factors Causing Nanocrystal Polydispersity .......................... 87

7.6.1. Laminar Flow Contribution to Nanocrystal Polydispersity ....................... 88

7.6.2. Nucleation Method Contribution to Nanocrystal Polydispersity................ 88

7.7. Investigation of Polydispersity at Low Residence Times (Spectral Tail) ......... 89

7.7.1. Analysis of the Effects of Thermal Gradients on Nanocrystal 


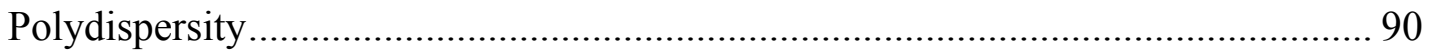

7.7.1.1. COMSOL Thermal Modeling Procedure …………….......................... 90

7.7.1.2. COMSOL Thermal Modeling Results ............................................... 92

7.7.2. Contribution of Fluid Resistance to Nanocrystal Polydispersity .................. 94

7.7.2.1. COMSOL Fluid Velocity Field Modeling............................................ 95

7.7.3. Stainless Steel Reactor and the Spectral Tail............................................... 96

7.7.4. Analysis of Reactor Surface Roughness Using SEM ................................... 96

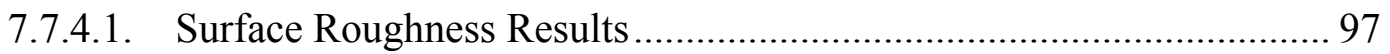

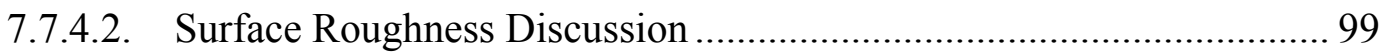

7.8. Physical Characterization of Quantum Dot Size Distribution ........................... 99

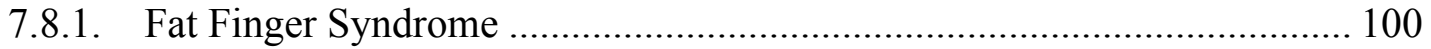

7.8.2. AFM Results and Discussion................................................................. 100

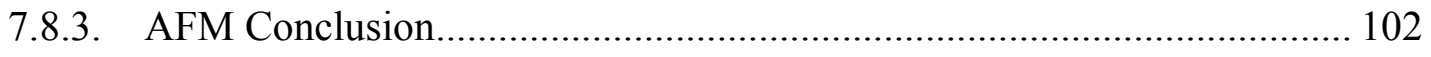

7.9. Relationship of Nucleation and Growth Theory to Microfluidic Reactor

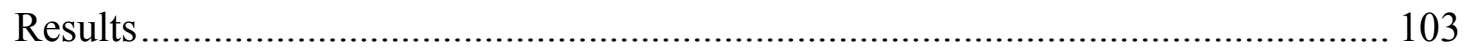

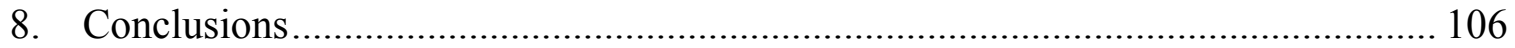

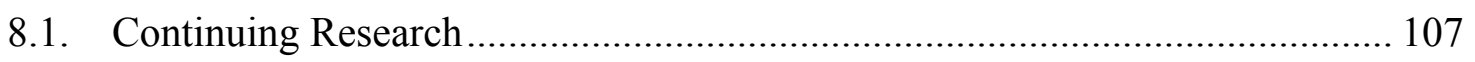

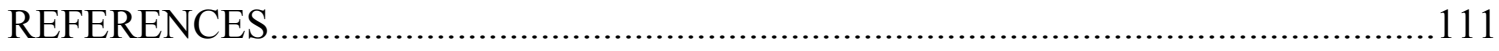

APPENDICES

Appendix A Microfluidic Quantum Dot Synthesis Required Supplies....... 115

Appendix B Quantum Dot Standard Operating Procedure (SOP)............... 117

Appendix C MATLAB Code $\quad$.................................................................... 130

Appendix D 316 Stainless Steel Reactor Two-Dimensional Spectral 
Profiles By Trial.

Appendix E 316 Stainless Steel Reactor Three-Dimensional

Topographic Spectral Profiles By Trial............................... 135

Appendix F TEFLON ${ }^{\circledR}$ Reactor Two-Dimensional Spectral Profiles

By Trial................................................................ 137

Appendix G TEFLON ${ }^{\circledR}$ Reactor Three-Dimensional Spectral Profiles

By Trial............................................................ 138

Appendix H SEM Analysis Procedures................................................... 139

Appendix I Gwyddion Surface Roughness Test Parameters...................... 140

Appendix J AFM Experimental Methods ............................................. 141 


\section{LIST OF TABLES}

Table I: Properties of materials suitable for reactor construction ${ }^{37}$

Table II: Comparison of the thermal expansion coefficients for glass, silicon, and SU-8. 25

Table III: Selected flow rates to be analyzed in all subsequent trials 32

Table IV: Calculated standard deviation of center wavelength at tested flow rates. 40

Table V: Selected flow rates to be analyzed in all subsequent P.O.C reactor trials 50

Table VI: Average center wavelength for selected flow rates 62

Table VII: Calculated standard deviation of center wavelength at tested flow rates

Table VIII: Calculated standard deviation of center wavelength at tested flow rates excluding erroneous trials. 68

Table IX: Average center wavelength for selected flow rates 70

Table X: Calculated standard deviation of center wavelength at tested flow rates. 77

Table XI: Optimized TEFLON ${ }^{\circledR}$ reactor comparison of center wavelength standard deviation by flowrate for old and new octadecene solvent 78

Table XII: Average standard deviation across all flow rates for all three tested reactors.

Table XIII: Thermal properties of solid reactor tubing materials used in COMSOL modeling. $^{37}$

Table XIV: Surface roughness values for the optimized 316 stainless steel reactor and TEFLON ${ }^{\circledR}$ reactor. 98 


\section{LIST OF FIGURES}

Figure 1: As quantum dots increase in size from left to right, a spectral emissions shift occurs in the visible spectrum from blue to red. ${ }^{5}$

Figure 2: Evolution of atomic orbitals into molecular orbitals to form energy bands.

The point at which discrete energy levels become bands is the point at which quantum dots no longer exhibit quantum confinement behavior. ${ }^{8}$. 3

Figure 3: Depiction the radius between an electron-hole pair otherwise know as the Bohr Radius. The particle depicted is smaller than the Exciton Bohr radius and experiences a phenomenon known as quantum confinement. ${ }^{10}$ 4

Figure 4: Correlation of increasing particle size to different band gap energies and different emitted photons. ${ }^{12}$

Figure 5: Small volumes of precursor solutions allow for better heat transfer due to a high surface area to volume ratio. ${ }^{15}$

Figure 6: Diagram showing the complete process of sample analysis using FIA. ${ }^{21}$ 11

Figure 7: Nucleation and growth profile for semiconductor nanoparticles. ${ }^{27}$ 15

Figure 8: The SMA flow cell uses fiber optic cables to analyze incoming flows at a $90^{\circ}$ angle. $^{41}$

Figure 9: A block diagram of the experimental test setup. Note the pathways of light and reagent solution.

Figure 10: Photo of the test setup for synthesizing quantum dots 30

Figure 11: Acquisition control pannel depicting integration time, boxcar width, and scans to average (center). Dark and light spectrums (right) are used to calibrate the spectrometer. 
Figure 12: Example of data manipulation applied to all flow rates to obtain best-fit curves for spectral peaks and relative center wavelength. 36

Figure 13: Average of three trials Gauss Newton regressions at six selected flow rates. Note the left shift of the peaks with increasing flow rate. 37

Figure 14: A power function regression was used to derive an empirical equation relating flow rate to center wavelength. The error bars represent the standard deviation between all collective trials at the specified flow rates...... 38

Figure 15: Average particle diameter for each tested flow rate (Proof of Concept Reactor).

Figure 16: a) Preliminary light source setup with a LED attached to a cuvette chuck.

b) New light source with an alignment chuck and adapter.

Figure 17: a) Ethylene glycol chiller system. b) Copper heat exchanger coil wrapped around glass $50 \mathrm{ml}$ syringe

Figure 18: Nucleation and growth of chilled quantum dots and room temperature quantum dots over a time frame of four hours.

Figure 19: Precursor solution mixing setup

Figure 20: Acquisition controls depicting integration time, boxcar width, and scans to average. 52

Figure 21: SpectraSuite ${ }^{\circledR}$ save parameters. Note that a scan is saved every 10 seconds for a duration of 50 seconds. 53

Figure 22: File tree organizing data for MATLAB processing. Note the folder (In Folders) and the folder (Data Not In Sub Folders) 54 
Figure 23: Two-dimensional spectral profile plot. Note each line represents a different SpectraSuite ${ }^{\circledR}$ output file recorded for one synthesis trial. 55

Figure 24: Three-dimensional surface plot generated from aligning the spectral profiles from an entire synthesis trial. Note the shift of the topographic peak as flowrate increases.

Figure 25: Fourth order Gaussian regression (Red) on raw spectral data (Blue) for a flow rate of $0.15 \mathrm{ml} / \mathrm{min}$ on trial 5_5_14.

Figure 26: Fourth order Gaussian regression (Red) on raw spectral data (Blue). Note the two sub peaks that make up the main spectral peak. 58

Figure 27: Steel recrystallization jig with a 2.5-inch outer diameter. Note Phillips screws used to secure tubing. 60

Figure 28: View of average spectral profiles at selected flow rates for all eight trials. Note that the center wavelength for each peak were converted to RGB color values and included as the flow rate color. Each spectral peak's color is the RGB equivalent of the color the synthesized quantum dots fluoresce. .. 61

Figure 29: Normalized view of average spectral profiles of selected flow rates for all eight trials.

Figure 30: Center spectral peak wavelength plotted by trial for all reagent flowrates. Note: Trial 4_25_14 was run with half of the standard volume of the TOPSe precursor solution. Trial 4_19_14 was run at a reaction temperature of $230^{\circ} \mathrm{C}$ causing a shift in center wavelength of the spectral peaks at each flowrate. 
Figure 31: Relationship of flow rate to the average center wavelength for all trials. Note the error bars show one positive and negative standard deviation of center wavelength.

Figure 32: Average particle diameter for each tested flow rate (316 stainless steel reactor)

Figure 33: View of average spectral profiles at selected flow rates for all four trials. Note that the center wavelength for each peak was converted to RGB color values and included in the plot. Each spectral peak's color is the RGB equivalent of the color the synthesized quantum dots fluoresce.

Figure 34: Normalized view of average spectral profiles of selected flow rates for all four trials. 71

Figure 35: Quantum dot fluorescence peak $(618 \mathrm{~nm})$ at a flow rate of $0.05 \mathrm{ml} / \mathrm{min}$.

Note this synthesis occurred at a temperature of $265^{\circ} \mathrm{C}$ 72

Figure 36: Center spectral peak wavelength plotted by trial for all reagent flowrates. Note: Trial 5_28_14 and trial 6_21_14 were run with old octadecene while trial 6_23_14 and trial 6_24_14 were run with new octadecene. 73

Figure 37: Relationship of flow rate to the average center wavelength for all TEFLON $^{\circledR}$ reactor trials. Note the error bars show one positive and one negative standard deviation of center wavelength.................................. 74

Figure 38: Average particle diameter for each tested flow rate (TEFLON ${ }^{\circledR}$ reactor)....... 76 Figure 39: Comparison of microfluidic reactor "blue" to bulk synthesis "red" synthesized quantum dots. 81 
Figure 40: Photograph of every other sample from trial three. Note the relatively uniform shifts in center wavelength with decreasing flow rate.

Figure 41: Comparison of center wavelength and flowrate relationship between the optimized 316 stainless steel reactor and optimized TEFLON ${ }^{\circledR}$ reactor. Note that the TEFLON ${ }^{\circledR}$ reactor produces larger quantum dots with a higher center wavelength than the stainless steel reactor at the same flow rate. 85

Figure 42: Comparison of old source of octadecene to new source of octadecene 86

Figure 43: Secondary spectral peaks form a "spectral tail effect" as flow rate increases. The broadening of the spectral profile indicates increasing polydispersity. 89

Figure 44: Comparison of the inlet thermal gradient for both the optimized TEFLON $^{\circledR}$ and the optimized 316 stainless steel reactors at a flow rate of $2 \mathrm{ml} / \mathrm{min}$. Note fluid injection occurs at a $\mathrm{Z}$ coordinate of $0.0127 \mathrm{~m}$. a) Thermal gradient for TEFLON ${ }^{\circledR}$ reactor tubing. b) Temperature of precursor fluid in the center of the TEFLON ${ }^{\circledR}$ tubing. c) Thermal gradient for 316 stainless steel reactor tubing d) Temperature of precursor fluid in the center of the 316 stainless steel reactor tubing. Note that the $\mathrm{Z}$ coordinate axis is backwards, fluid enters at the right at and travels to $0 \mathrm{~m}$... 93

Figure 45: "Slug" resulting from pressure driven flow. Note the slug shape and distribution of quantum dots within the reactor channel. 94

Figure 46: a) Three-dimensional view of the fluid velocity field. Note the wire frame on the outside is the tubing. b) Two-dimensional view of fluid velocity 
field. Note the radius of the tubing is about $397 \mu \mathrm{m}$. The blue region is about $90 \mu \mathrm{m}$ wide. The green region is about $80 \mu \mathrm{m}$ wide and the red region is about $210 \mu \mathrm{m}$ wide.

Figure 47: a) SEM micrograph of the optimized TEFLON ${ }^{\circledR}$ reactor tubing at 95X magnification. (Note the smooth surface). b) SEM micrograph of the optimized 316 stainless steel reactor tubing at 95X magnification. (Note the oxide spalling off the sample surface)

Figure 48: a) Micrograph of TEFLON ${ }^{\circledR}$ tubing 450X magnification. Note striations formed during extrusion. b) Micrograph of TEFLON ${ }^{\circledR}$ tubing 2000X magnification. c) Micrograph of 316 stainless steel reactor tubing with an oxide chip that has begun to crack and spall off the tubing surface. d) Micrograph of 316 stainless steel reactor tubing showing preferential etching along grain boundaries. 98

Figure 49: As the AFM tip approaches a quantum dot the side of the tip interacts with the quantum dot (1). The tip measures the apex of the quantum dot (2). The measured profile is distorted because of the interactions of the side of the tip and the quantum dot (3). (R. Gregoriev, personal communication, June 18, 2014) 100

Figure 50: Asylum AFM quantum dot measurements on 1" silicon wafer. a) Height profile of 11 scans. b) Micrograph of scan area. Note sizes range from about $1.5 \mathrm{~nm}$ to $12 \mathrm{~nm}$. (R. Gregoriev, personal communication, June 18, 2014) 
Figure 51: Asylum AFM baseline sample surface roughness analysis. Note residual quantum dots after cleaning. a) Height profile of 8 scans. b) Micrograph of scan area. Note sizes range from about $1.8 \mathrm{~nm}$ to $6 \mathrm{~nm}$. (R. Gregoriev, personal communication, June 18, 2014) 102

Figure 52: Average quantum dot radius as a function of residence time for all optimized reactor trials. The slope of red line represents the rate of particle growth at low residence times. The green line, blue line, and purple lines respectively represent decreasing growth rate with increasing residence time.

Figure 53: Quantum dot radius as a function of time in the heated section of the reactor. Note the curve at $240^{\circ} \mathrm{C}$ that matches the synthesis temperature used in this thesis. ${ }^{57}$ 104

Figure 54: Multiphase flow microfluidic device. Note immiscible fluid drops within the channel. ${ }^{58}$ 109

\section{LIST OF EQUATIONS}

Equation 1: Reynolds number calculation. $\mathrm{V}=$ velocity, $\mathrm{D}=$ hydraulic diameter, $\mathrm{p}=$ density, $\mu=$ dynamic viscosity

Equation 2: Hagen-Poiseuille equation: $\Delta \mathrm{P}=$ pressure drop, $\mu_{\mathrm{d}}=$ liquid viscosity, $\mathrm{L}=$ channel length, $\mathrm{r}=$ channel radius, $\mathrm{v}=$ velocity of flow 8

Equation 3: Volumetric flow rate (Q) 9

Equation 4: Relationship of free energy of a system to interfacial energy per unit area $\left(\gamma_{\mathrm{SL})}\right.$ and bulk energy per unit volume $\left(\Delta \mathrm{G}_{\mathrm{r}}\right){ }^{32}$ 
Equation 5: Thermodynamic critical radius of nuclei. Note inverse proportionality to

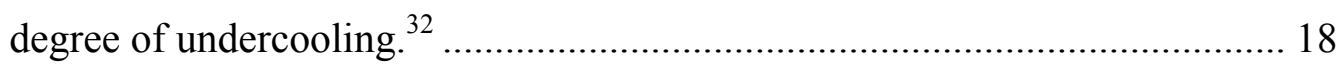

Equation 6: Change in free energy necessary to form stable nuclei. Note that the free energy change is inversely proportional to the degree of undercooling $(\Delta \mathrm{T})$

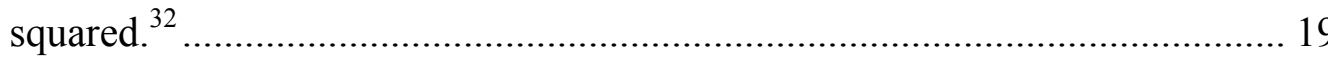

Equation 7: Critical radius relationship to supersaturation ratio $(\mathrm{S})^{28,33}$......................... 19

Equation 8: Power function relationship between flow rate and center wavelength......... 38

Equation 9: Empirical equation relating center wavelength to particle diameter............. 39

Equation 10: Time required to reach equilibrium at a given flow rate........................... 50

Equation 11: Power function relationship between average center wavelength and

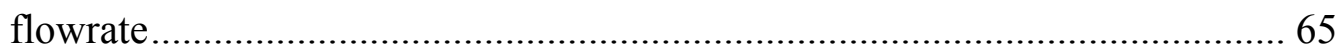

Equation 12: Optimized TEFLON ${ }^{\circledR}$ reactor power function relationship between average center wavelength and flowrate …………………........................... 75

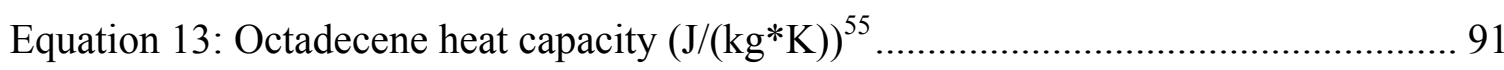

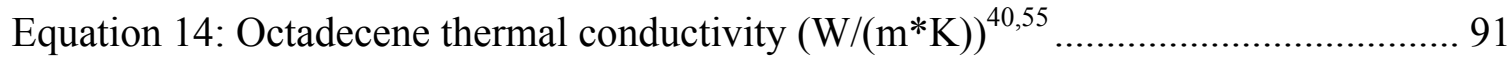

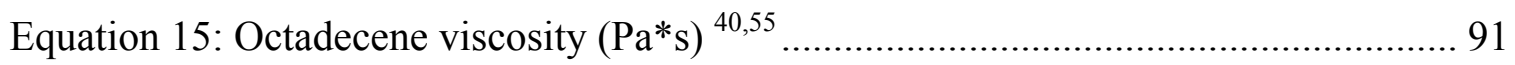




\section{INTRODUCTION}

\subsection{Problem Statement}

Colloidal semiconductor nanocrystals (NCs), also termed “quantum dots”, (QDs), consist of a Cadmium-Selenide (Cd-Se) core, composed of a few hundred to a few thousand atoms. ${ }^{1}$ Due to the phenomenon of quantum confinement, the size of the quantum dots influences their photoluminescence and band gap shift. ${ }^{2}$ The current process of fabricating quantum dots in the California Polytechnic State University (Cal Poly) Nanotechnology Lab is conducted on the bulk scale $(10 \mathrm{ml}-15 \mathrm{ml}){ }^{3}$ This bulk synthesis method, however, does not provide significant control over the variables that influence nucleation and growth, such as temperature and time. Additionally, cuvette fluorescent analysis is time-consuming, making real-time tuning of reaction parameters impossible. Consequently, a new method of synthesis was developed to yield quantum dots of welldefined sizes and fluorescence profiles. The use of a microfluidic reactor allows for precise control over factors that influence nucleation and growth due to the small volumes of reaction precursors involved. Constant flow analysis with a flow cell allows for real time monitoring of the formation of nanoparticles and tuning of reaction parameters. The goal of this thesis was to develop and characterize a microfluidic system capable of producing monodisperse quantum dot solutions while allowing for real time data analysis. 


\subsection{Quantum Dots}

Quantum dots are semiconductor nanocrystals typically 2-10nm in diameter. Because of their small size, quantum dots are considered unique in their ability to behave like a bulk material while preserving characteristics of individual atoms. ${ }^{4}$ Due to this unique phenomenon, quantum dots show great potential for use as spectral converters in solar cells, in lighting applications, and in biological imaging. ${ }^{1}$ Quantum dots allow for precise tuning of characteristics simply by altering their size. The relative sizes of quantum dots can be determined from their corresponding optical properties (Figure 1).

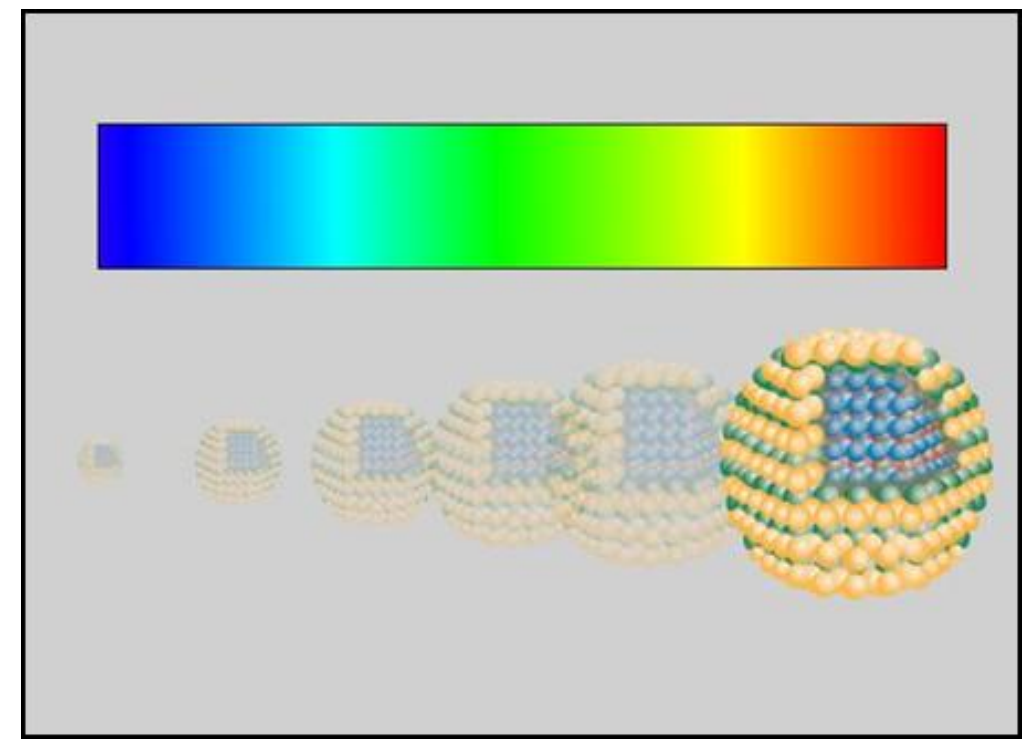

Figure 1: As quantum dots increase in size from left to right, a spectral emissions shift occurs in the visible spectrum from blue to red $^{5}$

To further understand the characteristics of quantum dots one must examine their atomic structure and the principal of quantum confinement. 


\subsubsection{Atomic Structure}

All atoms are effectively composed of positively charged nuclei surrounded by negatively charged "clouds" of electrons. These electron clouds are often thought of as shells surrounding the nucleus. Electron clouds are made up of discrete energy levels; the shells of electrons closer to the nucleus exist in a lower energy state than those farther away from the nucleus. When atoms are combined to form a molecule, the electron clouds combine to create molecular orbitals (MOs), which still contain discrete energy levels. ${ }^{6}$ When this model is extended to molecules made up of several atoms, the number of available MOs also increases. This phenomenon continues as more atoms are added until the energy level between MOs is virtually undistinguishable, forming a continuous energy band (Figure 2). The orbitals ultimately form a conduction band and a valence band. The energy between these two bands is called the band gap energy. ${ }^{7}$

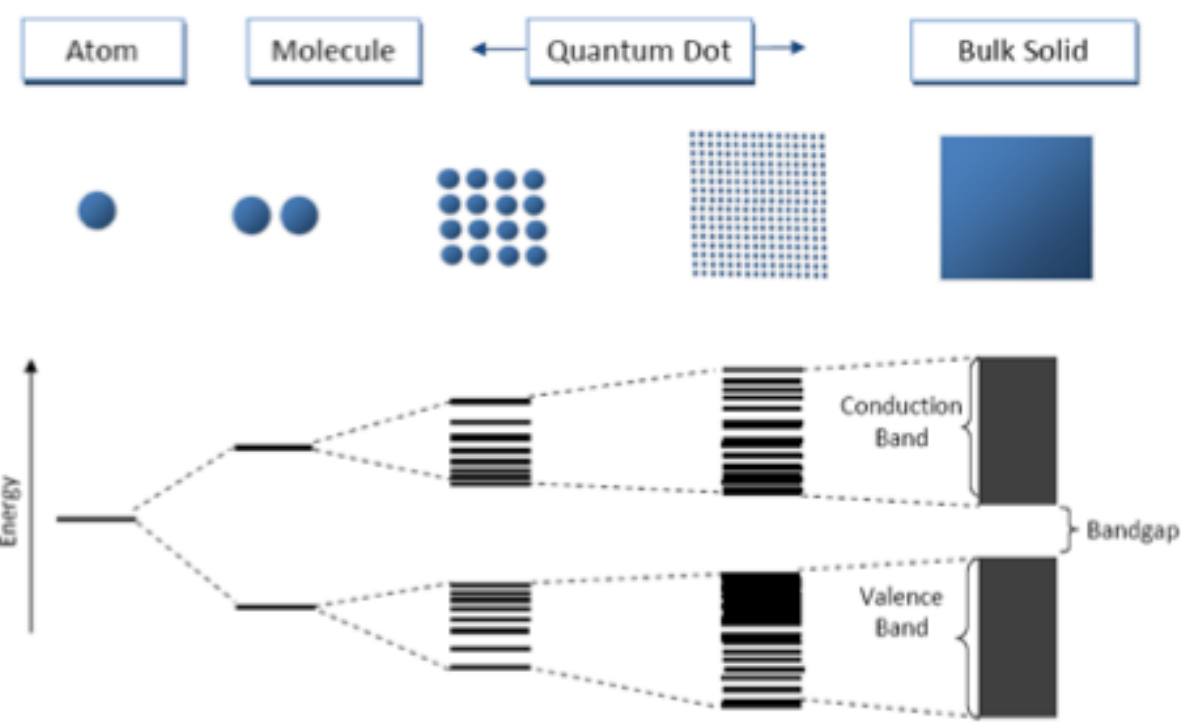

Figure 2: Evolution of atomic orbitals into molecular orbitals to form energy bands. The point at which discrete energy levels become bands is the point at which quantum dots no longer exhibit quantum confinement behavior. ${ }^{8}$ 
In bulk materials, the only way an electron can move from the valence band into the conduction band is to acquire as much, or more energy than the band gap energy. To jump the gap, an outside energy source is required. Heat, light or an applied voltage can provide the energy needed for an electron to jump the band gap. When an electron jumps to the conduction band, it leaves an electron "hole". The excited electron and its corresponding hole make up an exciton pair, where the physical distance between them is known as the Exciton Bohr Radius (Figure 3). ${ }^{9}$ In bulk materials, the Bohr radius is much smaller than the material itself, allowing the electron - hole pair to freely move across the atomic lattice. Since quantum dots are only $2-10 \mathrm{~nm}$ in size, they are smaller than the exciton Bohr radius; the electron - hole pairs are confined, which impacts their band gap energy.

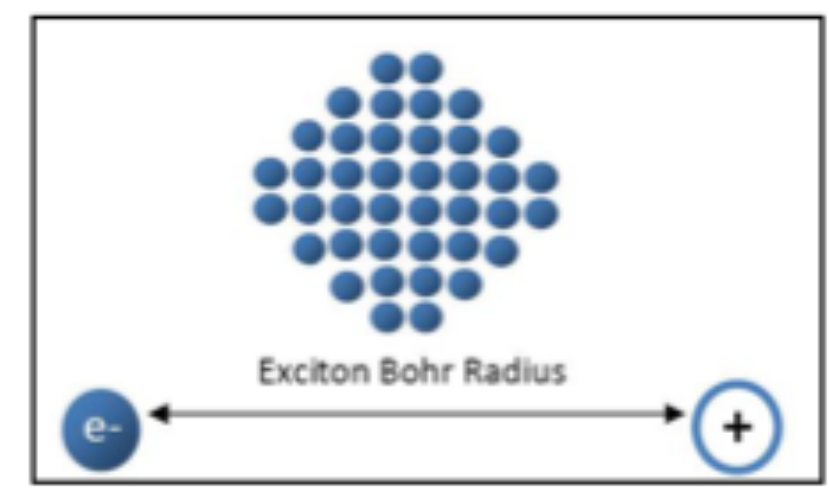

Figure 3: Depiction the radius between an electronhole pair otherwise know as the Bohr Radius. The particle depicted is smaller than the Exciton Bohr radius and experiences a phenomenon known as quantum confinement. ${ }^{10}$

Because quantum dots are so small, an exciton pairs movement is limited to the size of the cluster of atoms, and the exciton pair is unable to move the full natural radius. The 
surface of the quantum dots prevents electrons from reaching their full potential radius, resulting in a principle known as quantum confinement.

\subsubsection{Quantum Confinement}

The principle of quantum confinement arises as a result of changes in the density of available energy states. ${ }^{2}$ In bulk materials, the number of energy levels is virtually infinite, causing bands of energy. When dimensions are reduced to that of the Bohr radius, certain energy states become unavailable. When the radius of a cluster of atoms is smaller than the Bohr radius, as with quantum dots, only discrete energy levels remain. Due to quantum confinement, quantum dots can be engineered to create a desired pattern of energy levels. ${ }^{11}$ As atoms are added to a quantum dot, the number of discrete energy levels increases, causing a decrease in the band gap energy. The band gap energy can be effectively tuned by controlling the size of quantum dots. The band gap of quantum dots can be tuned to the range of energies of visible light resulting in fluorescence.

\subsubsection{Fluorescence}

Fluorescence is a phenomenon that makes quantum dots particularly desirable. Once an electron is excited by ultra-violet radiation, it jumps the band gap into the conduction band. The electron then relaxes from the conduction band releasing stored energy as a photon. The energy of this photon is equal to the band gap energy. Because the band gap energies of quantum dots can be engineered by increasing or decreasing particle size, the energy of the emitted photons can be tuned to produce a desired color (Figure 4). 


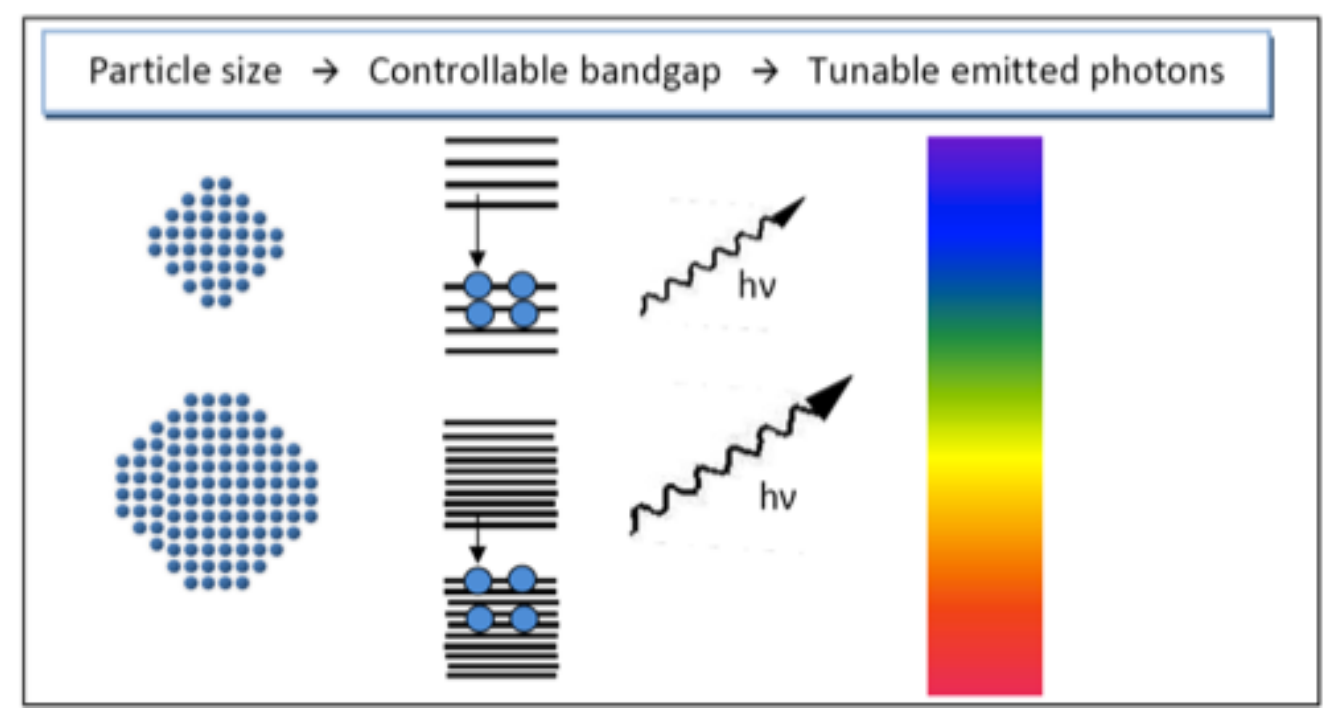

Figure 4: Correlation of increasing particle size to different band gap energies and different emitted photons. ${ }^{12}$

Fluorescence profiles provide rich information regarding biomolecules and their dynamics. They have been used to monitor polymerization processes, detect bases on DNA, measure diffusion coefficients, investigate binding sites of antibodies, and probe the internal polarity of proteins. ${ }^{13}$ Through an empirical relationship this fluorescence pattern can be used to effectively determine the size of a quantum dot.

\subsection{Microfluidics}

A microfluidic device allows for the manipulation of small volumes of fluids within an enclosure with dimensions typically less than several hundred microns. ${ }^{14}$ As the name implies, a microfluidic reactor mediates the reaction of small volumes of precursor liquid solutions. Microfluidic reactors are designed for continuous or segmented flows and offer many advantages over conventional bulk scale reactions. Typical advantages include 
improvements in heat transfer (Figure 5), energy efficiency, reaction speed, yield, safety, reliability, scalability, on-site/on-demand production, and real time data acquisition. ${ }^{3,15}$

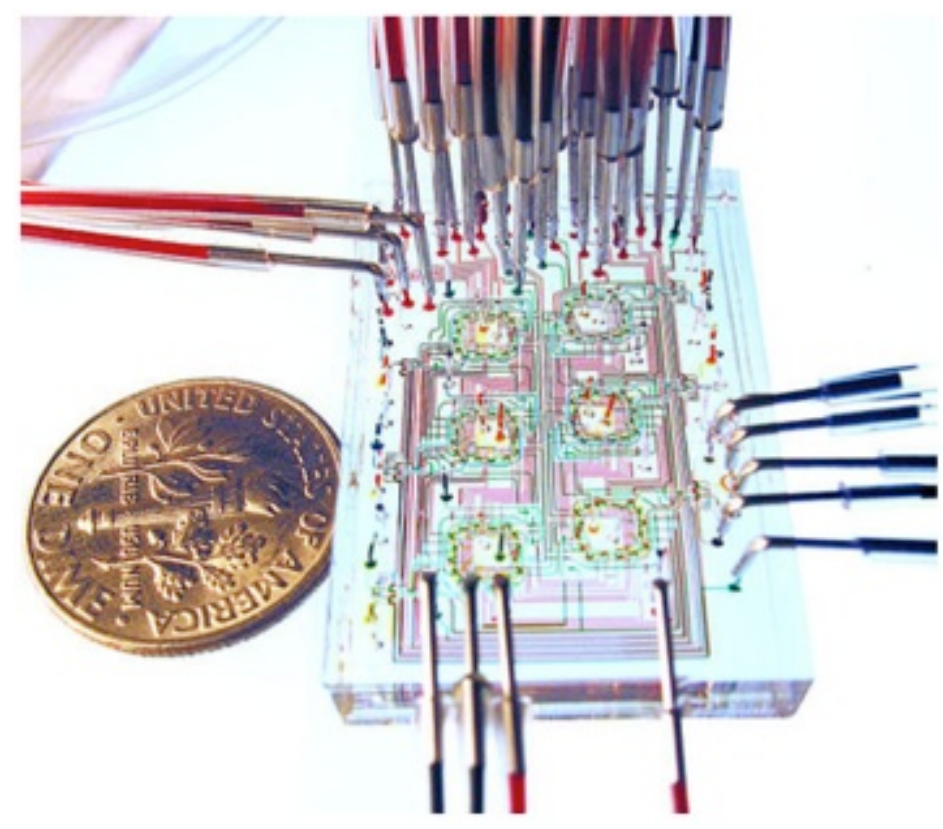

Figure 5: Small volumes of precursor solutions allow for better heat transfer due to a high surface area to volume ratio. ${ }^{15}$

Microfluidic device manufacturers use materials such as poly-dimethyl siloxane (PDMS), SU-8, glass, and silicon in microfluidic fabrication. This thesis focuses on the design and characterization of both a PTFE $\left(\right.$ TEFLON $\left.^{\circledR}\right)$ microfluidic reactor and a 316 stainless steel microfluidic reactor.

\subsubsection{Laminar Flow}

Laminar flow occurs when fluid flows in a channel without mixing or turbulence. In a two liquid system the liquid layers will flow alongside one another in parallel layers. The Reynolds number is a way to characterize the tendency of a flowing liquid phase to develop turbulence. Laminar flow occurs when the Reynolds number is less than $2000{ }^{16}$ 
The Reynolds number depends on the velocity of liquid flow within the channel, the diameter of the channel, and the viscosity of the liquid phase (Equation 1).

Equation 1: Reynolds number calculation. $V=$ velocity, $D=$ hydraulic diameter, $p=$ density, $\mu=$ dynamic viscosity

$$
R_{e}=\frac{\rho V D}{\mu_{d}}
$$

The calculated Reynolds number for the microfluidic reactor utilized in this study ranges from 0.39 at a $0.08 \mathrm{ml} / \mathrm{min}$ flow rate to 9.89 at a $2 \mathrm{ml} / \mathrm{min}$ flow rate. Therefore, it was assumed that a high degree of laminar flow occurs within this microfluidic system.

\subsubsection{Fluid Resistance and Pressure}

Fluid resistance causes pressure buildup within a microfluidic device. It results from a shear force between the liquid within a channel and the channel walls. The pressure buildup is equivalent to the difference in pressure across the device, from the pump to the outlet at atmospheric pressure. The pressure drop across a microfluidic device can be characterized by the Hagen-Poiseuille equation (Equation 2).

Equation 2: Hagen-Poiseuille equation: $\Delta \mathrm{P}=$ pressure drop, $\mu_{\mathrm{d}}=$ liquid viscosity, $\mathrm{L}=$ channel length, $r=$ channel radius, $v=$ velocity of flow

$$
\Delta P=\frac{8 \mu_{d} L v}{r^{2}}=Q_{\text {volumetric flow rate }} \bullet R_{\text {fluid resistance }}
$$

The Hagen-Poiseuille equation relates the pressure drop across the two ends of a microfluidic channel to the length and radius of the channel, liquid viscosity, and flow rate. This equation combines the volumetric flow rate and the fluid resistance to 
determine pressure within the device. Fluid resistance is caused by a shear force between the liquid and the walls of the channel, which leads to a pressure difference across the device. Pressure builds more quickly in devices with small channel diameters, high viscosities, large channel lengths, and high flow rates. This project was constrained by the SMA flow cell backpressure rating of 100 psi. Tubing with a diameter of $794 \mu \mathrm{m}$ was used to minimize pressure needed to push the precursor solution through the reactor.

\subsubsection{Volumetric Flow}

Volumetric flow describes the volume of fluid flowing through a channel per unit time (Equation 3). In this project the volumetric flow rate is a function of the syringe pump. The syringe pump can be adjusted to the desired flow rate, as long as the system does not experience failure due to backpressure.

Equation 3: Volumetric flow rate (Q)

$$
\mathrm{Q}\left(\frac{\mathrm{m}^{3}}{\mathrm{~s}}\right)=\mathrm{V}_{\text {flow velocity }}(\mathrm{m} / \mathrm{s}) * \mathrm{~A}_{\text {Area }}\left(\mathrm{m}^{2}\right)
$$

The syringe pump, which calculates the volumetric flow rate internally, was calibrated by inputting $27.94 \mathrm{~mm}$, the measured inner diameter of the $50 \mathrm{cc}$ syringe used in testing.

\subsubsection{Residence time}

The amount of time that the mixed precursor solution remained at reaction temperature is known as the residence time. In this microfluidic reactor, residence time is calculated as the length of the heated portion of the tubing multiplied by the velocity of the reagent 
through the tubing. Unlike bulk synthesis methods for nanoparticle production, a microfluidic reactor can precisely control the temperature as well as rapidly heat and cool the reagents, allowing for control of residence time down to sub-millisecond time frames. $^{14}$

\subsection{Flow Cell Technology}

Flow cell technology has been on the rise in the last few decades and is used in an evergrowing range of applications. High-pressure flow cells are often used for in-line analysis of high velocity liquid or gas streams in demanding industrial environments. ${ }^{17}$ These cells provide extremely short path lengths for controlled analysis without restricting sample flow. Flow cells allowing high sample throughput are used for environmental assays of water in nearby lakes and streams. With a typical sampling rate of two injections per minute, these flow cells allow for characterization of several hundred samples a day. ${ }^{18}$ Long path flow cells can also be utilized for trace element assays, with sensitivities up to the parts-per-trillion. The biotech industry utilizes flow cells for assays of biomolecules, bacteria, and living cells. Health industries conduct assays of active components in drug compounds using spectrophotometry in conjunction with a flow cell. ${ }^{18}$ The wide range of applications exemplifies the versatility of flow cell technology. All flow cell operations are derived from a principle known as flow injection analysis.

\subsubsection{Flow Injection Analysis (FIA)}

The concept of Flow Injection Analysis (FIA) was first introduced in the mid-seventies. ${ }^{19}$ Since then, the technology has grown from a tool for automation of serial assays to a 
method for enhancing the performance of spectroscopic and electrochemical instruments. ${ }^{20}$ FIA consists of injecting a sample into a carrier solution that is continuously moving at a constant flow rate. The target analyte is then taken through the reactor and enters the detector for analysis. The analytical readout is then collected for real time data analysis (Figure 6).

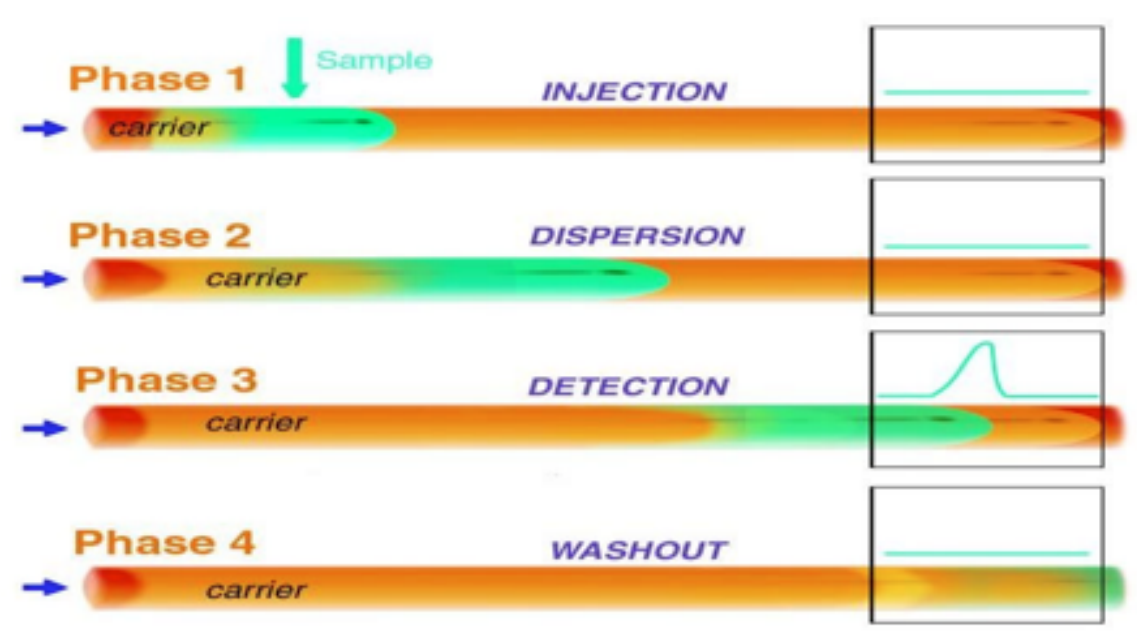

Figure 6: Diagram showing the complete process of sample analysis using FIA. ${ }^{21}$

The fast and intensive development of the FIA methodology was due to several factors essential for routine analytical determinations. Factors include limited sample consumption, short analysis time based on a transient signal measurement, on-line data acquisition of difficult operations of separation, and pre-concentration or physicochemical conversion of analytes into detectable species. ${ }^{19}$ Sequential injection analysis (SIA) was employed by the microfluidic reactor system utilized in this thesis. Sequential injection analysis is identical to FIA, but SIA uses segmented flow, allowing subsequent samples to be analyzed with different flow rates through the use of a syringe pump. 


\subsection{Broader Impacts}

\subsubsection{Sustainability}

Quantum dots are known to increase the efficiency of photovoltaic solar cells. Current research is being done on adding quantum dots to polymer solar cells at Cal Poly. In traditional silicon solar cells, only one electron can be excited from each photon, regardless of the photon's energy. For wavelengths of light that have energy above $1.1 \mathrm{ev}$, the energy difference after the excitation of the electron is lost as heat. Quantum dots have the unique property of producing more than one electron from each photon otherwise known as multiple exciton generation. ${ }^{22}$ Additionally, quantum dots can be tuned to many different band gap energies by varying their sizes. This allows for producing multi-junction solar cells that can make use of a greater amount of solar energy. ${ }^{23}$ Theoretically, an efficiency increase up to $42 \%$ is possible from a quantum dot based solar cell. $^{24}$

\subsubsection{Environmental Impact}

As nanotechnology continues to develop, possible health and environmental effects are coming to light. An important factor in determining environmental impact of a nanostructure is the product life cycle. For quantum dots, the life cycle is dependent on the life cycles of the chemicals used in quantum dot synthesis. Because the chemicals involved with quantum dots are environmentally harmful and carcinogenic, careful consideration must go into limiting waste of these chemicals. Researchers today are focusing on the effects of disposing products that contain nanoparticles such as quantum dots. Key concerns are related to how the nanocrystals move through soil and how they 
accumulate within plants and animals. ${ }^{25}$ As quantum dots continue to be produced in mass quantities, the effects of disposing the heavy-metal chemicals are an increasing concern. The aim of this thesis is to design a microfluidic reactor to produce quantum dots under tightly controlled conditions. Precise control over flow rate and reaction temperatures will limit waste over repeated experiments.

\subsubsection{Manufacturability}

The current process of synthesizing quantum dots in the Cal Poly Nanotechnology Lab is conducted through a multitude of steps, each requiring precise control over variables such as temperature and time. The focus of this project was to reduce the amount of variability between samples produced through the conventional bulk synthesis method at Cal Poly. Design efforts centered on the production of quantum dots of a precise size, increasing quantum dot fluorescent intensity, and decreasing the quantum dot size distributions typical of the currently utilized bulk synthesis method.

\subsubsection{Safety}

Quantum dots are made from heavy metals and toxic chemicals, such as cadmium and selenium, which pose risks to the health of the people handling them. Researchers are currently focused on the effects of biomedical applications involving quantum dots, such as in vivo imaging. Scientists have found that quantum dots break down and release their

toxins under mildly acidic environments. ${ }^{26}$ A relevant concern is that quantum dots could break down from acids in the body, releasing harmful chemicals into the bloodstream. Because nanotechnology is still an emerging technology, there is continuous research on 
possible safety hazards and long-term effects from quantum dot production. To minimize safety risks nitrile gloves, chemical aprons, splash proof safety glasses, and fume hoods were utilized. 


\section{BACKGROUND ON NUCLEATION AND GROWTH THEORY ${ }^{\dagger}$}

Nucleation and growth of semiconductor nanocrystals depend on a combination of kinetics and thermodynamics. Solid phase quantum dot nanocrystals form through a liquid solid phase transformation from a homogeneous supersaturated solution of reaction monomers. The transformation occurs in three distinct stages (Figure 7). ${ }^{27,28}$

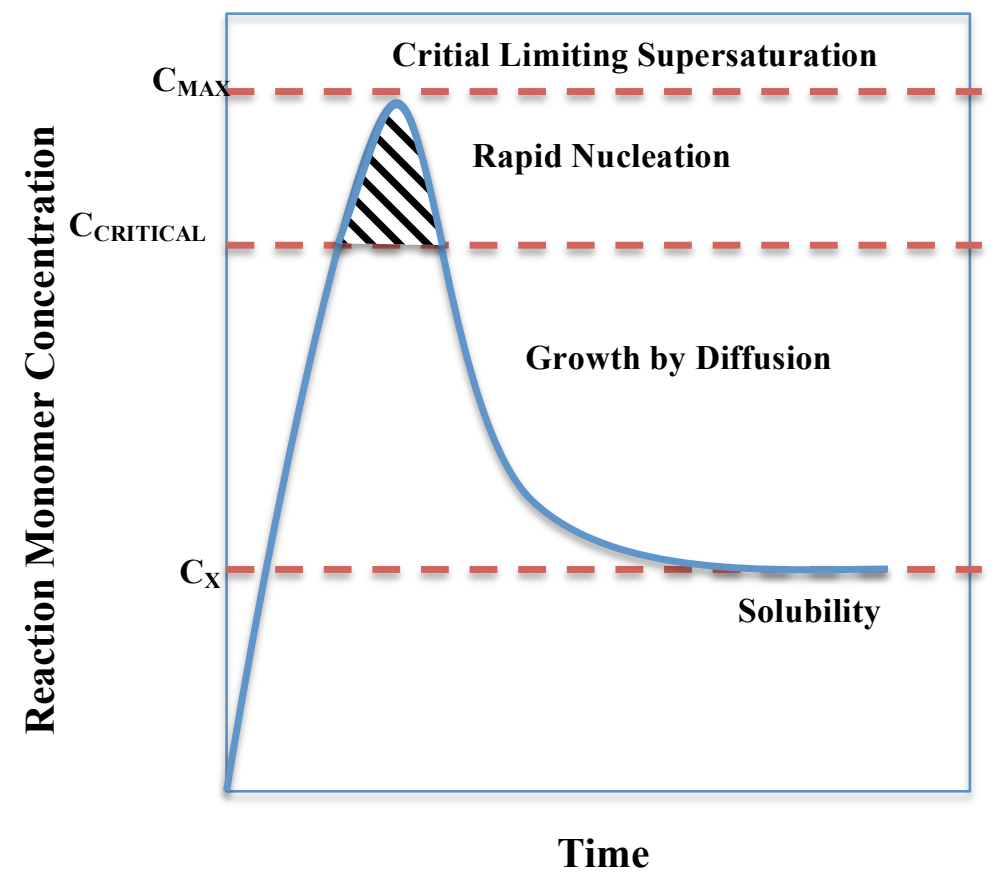

Figure 7: Nucleation and growth profile for semiconductor nanoparticles. ${ }^{27}$

Initially the reaction precursors dissociate, due to the high thermal energy of the system, to create a saturated solution of reaction monomers. Rapid nucleation begins after the solution becomes supersaturated with these monomers past a critical point. The nucleation phase transitions to the growth phase after the energy of the solution lowers below the critical point where the creation of new nuclei gives no energy benefit. During the growth phase solution remains supersaturated with reaction monomers and growth continues on existing nuclei. It is important to note that the initial nucleation burst does 
not occur at one instant in time but instead develops concurrently with the growth of nuclei. This phenomenon contributes to semiconductor nanocrystal polydispersity. Having a long growth period in relation to the nucleation period minimizes the polydispersity of a sample. However, concentrations of reaction monomers decrease as the reaction proceeds and eventually small nanocrystals start to dissolve while larger nanocrystals continue to grow. ${ }^{29}$ This process known as Ostwald ripening contributes to polydispersity and consequently broadening spectral profiles at longer residence times. $^{30,31}$

\subsection{Classical Nucleation Theory}

Due to the shear number of variables involved in a nanocrystal synthesis process, predictions of nucleation and growth characteristics are better accomplished through the use of empirically generated equations. Despite this, classical nucleation theory can be used as a rough model to understand trends caused by nucleation and growth kinetics.

\subsubsection{Homogeneous Nucleation}

Homogeneous nucleation describes spontaneous nucleation at random sites within a solution. New phases spontaneously form in the bulk solution. The classical model of nucleation and growth assumes homogeneous nucleation. ${ }^{32}$ 


\subsection{Nucleation and Growth Thermodynamics}

The thermodynamics of a process predict the most stable equilibrium phase. Given enough time all processes proceed to equilibrium. Thermodynamically speaking, a new phase forms when the new phase provides a lower energy state for the bulk system. The energy state of a system when looking at nucleation and growth is a combination of interface energy $\gamma_{\mathrm{SL}}$ per unit volume and bulk energy $\Delta \mathrm{G}_{\mathrm{r}}$ per unit volume. Interface energy describes the energy required in the creation of a new surface while bulk energy describes changes in free energy inherent in the structure of different phases i.e. solid to liquid phase transformations. If a spherical precipitate minimizes the combination of these two terms in respect to the rest of the solution it will be stable and grow, provided that the atomic mobility of the system is conducive to growth (Equation 4).

Equation 4: Relationship of free energy of a system to interfacial energy per unit area $\left(\gamma_{\mathrm{SL})}\right.$ and bulk energy per unit volume $\left(\Delta \mathbf{G}_{\mathbf{r}}\right)$. $^{32}$

$$
\Delta G(r)=\left(4 \pi r^{2}\right) \gamma_{s L}+\frac{4}{3} \pi r^{3}\left(\Delta G_{r}\right)
$$

The total interfacial energy of a particle is a function of the surface area and the interfacial energy per unit area $\left(\gamma_{\mathrm{SL}}\right)$. As a particle begins to form, due to its small size the molecular interactions are dominated by the energy to create the new surface (high surface area to volume ratio). At this point homogeneous nucleation requires sufficient undercooling to justify the energy required to create the interface of a new phase. Heterogeneous nucleation, discussed later in this section, minimizes this energy by piggybacking off an existing surface thus reducing the interfacial energy barrier for nucleation. Once the particle has reached a critical size, however, spontaneous growth or 
dissolution depends on the bulk free energy of the system. In the case of spherical nuclei, this critical size is defined by the radius of the nuclei. This critical radius is obtained by differentiating (Equation 4) with respect to particle radius and solving for the radius (Equation 5).

\section{Equation 5: Thermodynamic critical radius of nuclei. Note inverse proportionality to degree of undercooling. ${ }^{32}$}

$$
r^{*}=\frac{\left(-2 \gamma_{S L}\right) T_{E}}{\Delta H_{r} \Delta T} \quad r^{*} \alpha \frac{1}{\Delta T}
$$

The critical radius is the size a particle must grow to achieve stability. Below the critical radius the particle is know as a subcritical nuclei and it will spontaneously dissolve back into solution to decrease free energy. Above the critical radius, only growth of the particle can decrease free energy of the system. This means that particles smaller than this grow more slowly because the free energy of the reaction is inversely proportional to the square of the difference in temperature between the solidus temperature and the reaction temperature. This difference in temperature is known as the degree of undercooling. By plugging in the equation for the critical radius (Equation 4) into the equation for free energy (Equation 5) the relationship to undercooling $(\Delta \mathrm{T})$ becomes apparent (Equation 6). The thermal energy of the system must be low enough to provide sufficient undercooling to nucleate nanocrystals with a critical radius of $1-10 \mathrm{~nm}$ in diameter. As no data could be found on the solidus temperature for the precursor solution used for this synthesis, this equation provided a guide on the importance of temperature fluctuations on nucleation and growth. The reaction temperature range $\left(235-240^{\circ} \mathrm{C}\right)$ however, was obtained from literature. ${ }^{27}$ 
Equation 6: Change in free energy necessary to form stable nuclei. Note that the free energy change is inversely proportional to the degree of undercooling $(\Delta \mathrm{T})$ squared. ${ }^{32}$

$$
\Delta G^{*}=\frac{16\left(\gamma_{S L}\right)^{3} T_{E}^{2}}{3\left(\Delta H_{r}\right)^{2}} \times \frac{1}{(\Delta T)^{2}} \quad \Delta G^{*} \alpha \frac{1}{(\Delta T)^{2}}
$$

The nucleation and growth reaction occurs at constant temperature throughout the reactor so the degree of undercooling remains constant. Yet the degree of supersaturation of the reagent solution with reaction monomers is dynamic. As precipitates nucleate and grow the concentration of reaction monomers in the bulk solution is depleted. This affects the critical radius. The relationship between critical and degree of supersaturation is show in Equation 7 below.

Equation 7: Critical radius relationship to supersaturation ratio $(\mathbf{S})^{28,33}$

$$
r_{c}=\frac{2 v_{m} \gamma}{R T \ln (S)} \quad S=\frac{C^{\prime}}{C}
$$

$\mathrm{C}^{\prime}$ is the concentration of reaction monomers in solution surrounding the nuclei, whereas

$\mathrm{C}$ is the concentration of reaction monomers in the bulk solution. As the reaction progresses the concentration of reaction monomers in the bulk solution decreases causing the supersaturation ratio to decrease and the critical radius to increase.

\subsection{Kinetics}

Reaction kinetics describe the non-equilibrium rate at which atomic and molecular processes proceed to equilibrium. The two main parameters when dealing with molecular kinetics are time and temperature. Molecular kinetics play a large role in processes that 
require mass transfer, such as nucleation and growth reactions. The thermal energy of a system affects the amount of nucleation, the mobility of atoms in solution, and reaction monomer solubility. In kinetic processes, atoms spontaneously form and break bonds with one another. By increasing the thermal energy of a system, more energy exists to form and break atomic bonds, and for atoms to move more quickly. This causes reactions to progress more quickly to an equilibrium state.

\subsubsection{Precursor Solution Kinetics}

The precursor solutions used in this synthesis are cadmium oleate and TOP-Se dissolved in the solvent octadecene. The solubility limit for these solutions is temperature dependent. Reaction monomers do not fully dissociate until they are raised to the reaction temperature.

\subsubsection{Nucleation and Growth Kinetics}

In regards to nanocrystal nucleation and growth reactions, raising the temperature of the solution results in several outcomes. Atoms collide into each other more often and thus more nucleates are formed in a given time. Greater atomic mobility increases the rate at which reaction monomers are transported to the nuclei (diffusion). The increased thermal energy provides energy needed to overcome activation energy barriers in the incorporation of new atoms into growing nanocrystals (form bonds). 


\subsubsection{Diffusion Controlled Growth}

In diffusion-controlled growth the surface reaction, where monomers are incorporated into the bulk nanocrystal surface, occurs rapidly enough that the transport of new monomers to the sample surface dictates the rate of the growth reaction. The diffusion process is an Arrhenius process meaning that the molecular interactions are temperature dependent. Increasing temperature increases atomic mobility, which increases the diffusion rate. If the mobility of monomers in solution is lower than the rate of monomer incorporation to the crystalline lattice the concentration of monomers in the solution surrounding the nuclei decreases. This results in a concentration gradient with respect to the bulk solution inducing transport of monomers to the surface of the nuclei. Mass transport from solution is limited by the speed at which molecules can move through the

solution. ${ }^{34}$ Assuming reaction-controlled growth, the nucleation and growth reaction for quantum dot synthesis proceeds as follows:

Concentration of reaction monomers in the reagent solution decreases as the growth reaction progresses. Initially, when the reagent enters the reactor it is supersaturated with reaction monomers. After the initial burst of nucleation and growth, the concentration of reaction monomers begins to decrease around the nuclei. This forms a concentration gradient where reaction monomers are transported by diffusion to the growing nuclei. Essentially, the rate of growth of the nuclei is controlled by the diffusion rate of monomers through solution. 


\subsubsection{Reaction Controlled Growth}

In reaction-controlled growth diffusion occurs faster than the reaction to incorporate new monomers into the crystalline lattice. A possible explanation for the incorporation reaction occurring slowly is that the activation energy required for the process to occur has not been met. Higher reaction temperatures provide more free energy to activate the addition of reaction monomers to the nanocrystal lattice. Additionally, in reactioncontrolled growth larger nanocrystals will grow more rapidly as there is a greater surface area for monomer incorporation to occur.

\subsection{Heterogeneous Nucleation}

Heterogeneous nucleation relies on an external surface to provide a nucleation site. By piggybacking off of existing surfaces the energy required for a particle to grow past the critical radius and become stable is lessened. Essentially surfaces catalyze the nucleation reaction. The contact angle describes the angle formed between the nucleus and surface. While the critical radius stays relatively the same between heterogeneous and homogeneous nucleation the critical volume of the nucleus is usually smaller for heterogeneous nucleation due to the wetting effect. The smaller the contact angle (greater surface wetting) the lower the free energy change, and the lower the nucleation barrier. As such heterogeneous nucleation occurs preferentially when a surface is present to catalyze the reaction. 


\section{PROOF OF CONCEPT (P.O.C) MICROFLUIDIC REACTOR SYSTEM}

A manufacturing process was needed to produce quantum dots with well-defined spectral peaks across a broad range of wavelengths at Cal Poly. To achieve a controlled synthesis process, real-time data analysis was necessary. A microfluidic system was determined to be the most viable synthesis option because a microfluidic reactor coupled with a fluorescence flow cell allows for simultaneous characterization and synthesis.

\subsection{Microfluidic Reactor}

A microfluidic device was required to synthesize quantum dots at temperatures up to $240^{\circ} \mathrm{C}$ while remaining unaffected by the solvents used in the reaction process.

Additionally the reactor had to be heated to synthesis temperature without subjecting the flow cell to reaction temperature. Two designs were investigated in reactor fabrication. The first was a microfluidic chip that would rest directly on a hot plate and the second was a coil of small gauge tubing immersed in a thermal bath. In both designs a digital hot plate was used to provide thermal energy to the reactor.

\subsubsection{Materials Selection}

Several materials were investigated for chemical compatibility and thermal stability. Synthesis of CdSe quantum dots has been conducted at temperatures ranging from $180^{\circ} \mathrm{C}$ to $320^{\circ} \mathrm{C}^{35} 235^{\circ} \mathrm{C}$ was chosen for characterization of this reactor. Additionally, The materials have to be chemically resistant to octadecene, a hazardous alkene, at reaction temperatures. ${ }^{36}$ Using CES software, the six materials shown in Table I were selected based on the functional requirements listed above as well as cost and manufacturability. 
Table I: Properties of materials suitable for reactor construction ${ }^{37}$

\begin{tabular}{|c|c|c|c|}
\hline Material & $\begin{array}{c}\text { Service Temperature } \\
\left({ }^{\circ} \mathrm{C}\right)\end{array}$ & Chemical Stability & Cost \\
\hline SU-8 2050 & 250 & Excellent & $1.6(\mathrm{USD} / \mathrm{ml})$ \\
\hline $\begin{array}{c}\text { Borosilicate } \\
\text { Glass }\end{array}$ & 500 & $\begin{array}{c}\text { Strong acids: Excellent } \\
\text { Strong alkalis: Excellent } \\
\text { Organic Solvents: Excellent }\end{array}$ & $\begin{array}{c}9.84(\mathrm{USD} / \mathrm{Kg}) \\
\text { Manufacturing } \\
\text { Cost } \$ \$ \$ \$ \$\end{array}$ \\
\hline $\begin{array}{c}\text { TEFLON } \\
(\mathrm{PTFE})\end{array}$ & 271 & $\begin{array}{c}\text { Strong acids: Excellent } \\
\text { Strong alkalis: Excellent } \\
\text { Organic Solvents: Excellent }\end{array}$ & $16.9(\mathrm{USD} / \mathrm{Kg})$ \\
\hline $\begin{array}{c}316 \\
\text { Stainless } \\
\text { Steel }\end{array}$ & 750 & $\begin{array}{c}\text { Strong acids: Acceptable } \\
\text { Strong alkalis: Excellent } \\
\text { Organic Solvents: Excellent }\end{array}$ & $5.42(\mathrm{USD} / \mathrm{Kg})$ \\
\hline Silicon & $>1200$ & $\begin{array}{c}\text { Strong acids: Excellent } \\
\text { Strong alkalis: Excellent } \\
\text { Organic Solvents: Excellent }\end{array}$ & $\begin{array}{c}20 \\
(\mathrm{USD} / \mathrm{Wafer})\end{array}$ \\
\hline
\end{tabular}

Due to availability and ease of manufacturing SU-8 soft lithography was initially selected to create a prototype microfluidic reactor.

\subsubsection{SU-8 Based Reactor}

Initially the microfluidic reactors construction relied on SU-8, an epoxy-based negative photoresist with outstanding chemical compatibility and a high working temperature. ${ }^{38}$ SU-8 was patterned and developed in the desired channel shape and bonded to a Pyrex wafer coated with an additional layer of SU-8. This chip was designed to rest directly on a hotplate at a reaction temperature of $235^{\circ} \mathrm{C}$. A working T-mixer chip in this configuration was fabricated to test the chemical compatibility at synthesis temperatures. Once the chip was raised to temperature and the reactant solution was injected, the SU-8 began to crack and delaminate. The delamination was theorized to result from differences in thermal expansion between SU-8, glass, and silicon (Table II). 
Table II: Comparison of the thermal expansion coefficients for glass, silicon, and SU-8

\begin{tabular}{|c|c|}
\hline Material & Thermal Expansion Coeff $\left(\right.$ Strain $\left./{ }^{\circ} \mathbf{C}\right)$ \\
\hline SU-8 & $58 \mathrm{E}^{-6}-90 \mathrm{E}^{-6}$ \\
\hline Glass & $3.2 \mathrm{E}^{-6}-4 \mathrm{E}^{-6}$ \\
\hline Silicon & $2 \mathrm{E}^{-6}-3.2 \mathrm{E}^{-6}$ \\
\hline
\end{tabular}

From this experiment SU-8 soft lithography was deemed impractical in the construction of a microfluidic device for synthesis of quantum dots and new methods of fabrication were investigated.

\subsubsection{Patterned Silicon Wafer and Pyrex Wafer Reactor}

A process called anodic bonding can be used to bond a patterned silicon wafer to a Pyrex ${ }^{\circledR}$ wafer. ${ }^{39}$ Despite successful trials outlined in a previous Cal Poly thesis, considerable safety risk of dealing with exposed high voltage electrodes made this option unfeasible.

\subsubsection{Prefabricated Tubing Reactor}

The previously outlined fabrication limitations were overcome by using prefabricated tubing as a reactor. Glass tubing, TEFLON ${ }^{\circledR}$ tubing and stainless steel tubing were investigated. It was determined that third party fabrication of a glass capillary tubing coil was cost prohibitive. Stainless steel tubing was another viable option but was not easily bent to shape to fit in a high temperature silicon oil reactor bath. A 6-inch length of 1/16inch inner diameter PTFE tubing was used to validate the tubing reactor concept. 


\subsubsection{PTFE Tubing Reactor Coil}

Polytetrafluoroethylene (PTFE) tubing appeared the best choice to minimizing both cost and fabrication time. PTFE has a working temperature of $271^{\circ} \mathrm{C}$, it is resistant to octadecene, a hazardous alkene solution, and it is flexible, making it ideal for this application. ${ }^{40}$ PTFE tubing with a 1/32-inch inner-diameter and 1/16-inch outer-diameter was selected to match the ferrule connectors used in the Ocean Optics ${ }^{\circledR}$ fluorescence flow cell. A coil 30-inch long was made to fit in a 4-inch high temperature silicon oil bath.

\subsection{Flow Cell}

Initially, the flow cell was intended for characterization of various fluorescent solutions, such as fluorescein, using the concept of flow injection analysis with a syringe pump. The goal was to accurately characterize dynamic flows at the micro-scale and compare the results to conventional cuvette analysis. After determining the capabilities of the flow cell, the flow cell was incorporated into a quantum dot synthesis process. This allowed for simultaneous synthesis and characterization. Another motivation for combining these concepts was that no literature on a fluorescence flow cell operating in conjunction with a cadmium-selenide quantum dot synthesis process was found. To capture the fluorescence profiles at various flow rates using real-time data acquisition, a flow cell was essential.

The flow cell chosen for this particular experiment was an Ocean Optics ${ }^{\circledR}$ Sub-Multi Assembly (SMA) fluorescence flow cell with a $6 \mathrm{~mm}$ path length and $30 \mu \mathrm{L}$ internal volume (Figure 8). This particular flow cell is widely accepted for its versatility and ease of use. 


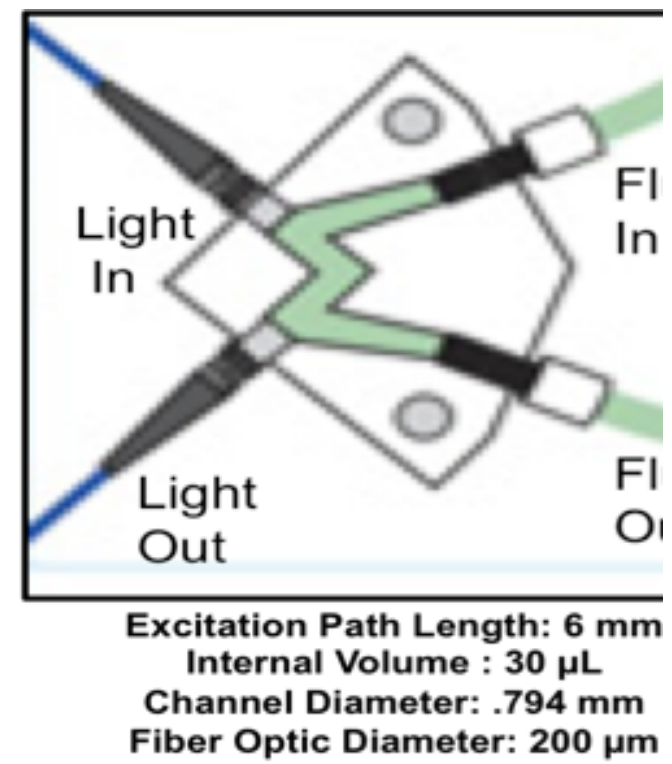

Figure 8: The SMA flow cell uses fiber optic cables to analyze incoming flows at a $90^{\circ}$ angle. ${ }^{41}$

To prevent hazardous chemical reactions, PTFE nuts and ferrules were chosen to secure connections with the microfluidic tubing and the flow cell. An Ultem ${ }^{\circledR}$ housing was used for the flow cell because of its compatibility with alkenes similar to octadecene. ${ }^{42}$

\subsubsection{Flow Cell Light Source}

In order to cause fluorescence, a ThorLabs ultraviolet light source was used to excite the electrons in the quantum dot solutions. Fiber optic cables were used to transfer light from the light source to flow cell, and from the flow cell to an Ocean Optics ${ }^{\circledR}$ USB4000 Spectrometer for data acquisition. The Fiber optic cable screwed into a sleeve, which was aligned with a light source through a cuvette chuck. A large portion of the ultraviolet light was lost through the oversized opening and the cuvette chuck degrading fluorescent intensity. 


\subsection{Design Integration and Optimization}

For this project design iterations were created and modified to simulate the conventional Cal Poly bulk quantum dots synthesis process. The aim was to re-produce the nucleation and growth conditions achieved by the established bulk synthesis reactions. Initially, a piece of PTFE tubing 6-inches in length was submerged in an oil bath at $235^{\circ} \mathrm{C}$.

However, testing revealed 6-inches of tubing was ineffective in obtaining the required residence times for synthesis of a broad spectrum of quantum dots. The second design incorporated a coil of PTFE tubing 30-inches in length submerged in a hot oil bath at $235^{\circ} \mathrm{C}$, followed by 9 -inches of tubing submerged in a water quench at $25^{\circ} \mathrm{C}$. The quantum dot solution then entered the flow cell for characterization before being collected into various sampling vials (Figure 9). This design was used with initial testing as a Proof of Concept. The third and final design iteration involves slight modifications to the second design including increasing synthesis temperature to $240^{\circ} \mathrm{C}$, a new excitation light source and light source fixture, and a chilling system to chill the precursor solution at the syringe pump before it is pumped through the reactor to limit QD growth at room temperature. 


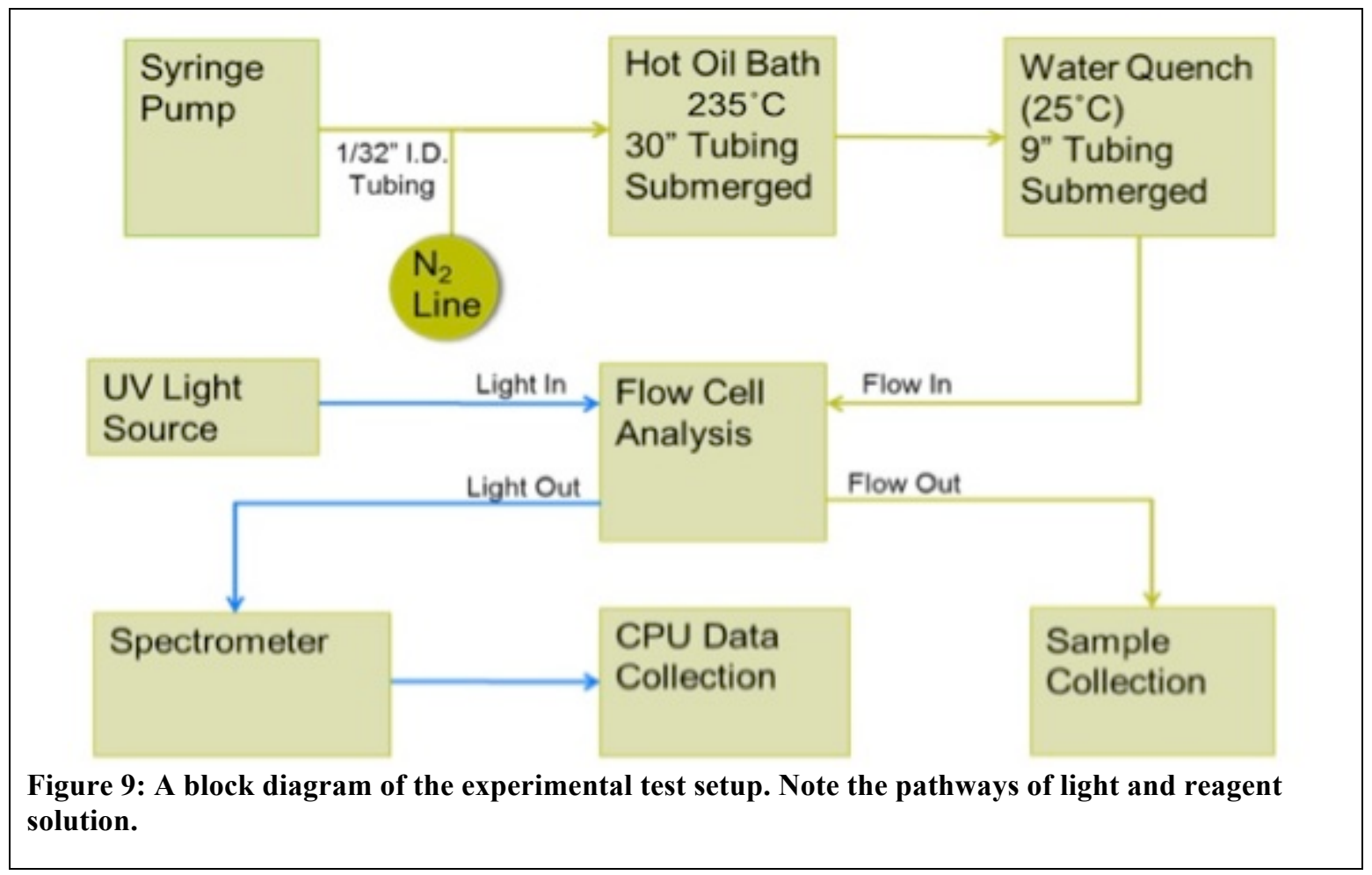

The two key variables in this synthesis method were temperature and flow rate. Because the flow rate was known and accurate, maintaining the temperature was a key concern. In order to maintain consistency of these temperatures, a Cimarec ${ }^{\circledR}$ hot plate was used in conjunction with an Extec ${ }^{\circledR}$ thermocouple to keep the oil bath at $235^{\circ} \mathrm{C}$. The water quench apparatus was filled with room temperature deionized water at $25^{\circ} \mathrm{C}$

\subsubsection{Laboratory Test Setup}

In order to accurately pump the quantum dot solution with a known volumetric flow, a New Era ${ }^{\circledR}$ model NE-300 syringe pump was used. Subsequent samples at various flow rates were attainable with the push of a button. A nitrogen line was connected to the three-way valve attached to the syringe. The nitrogen lines pressure was set to 4 psi in order to purge the reactor system between sampling events without causing excess 
backpressure. The PTFE tubing was then submerged in a silicon-based oil bath at $235^{\circ} \mathrm{C}$, followed by a quench of deionized water at $25^{\circ} \mathrm{C}$. The solution then entered the SMA fluorescence flow cell for characterization. In order to properly characterize the fluorescence profiles of quantum dots, a Thor Labs ${ }^{\circledR}$ LED light source was used to emit light at $385 \mathrm{~nm}$. These photons were carried through $200 \mu \mathrm{m}$ diameter SMA fiber optic cables attached to the flow cell and then to an Ocean Optics ${ }^{\circledR}$ USB4000 spectrometer for data collection (Figure 10).

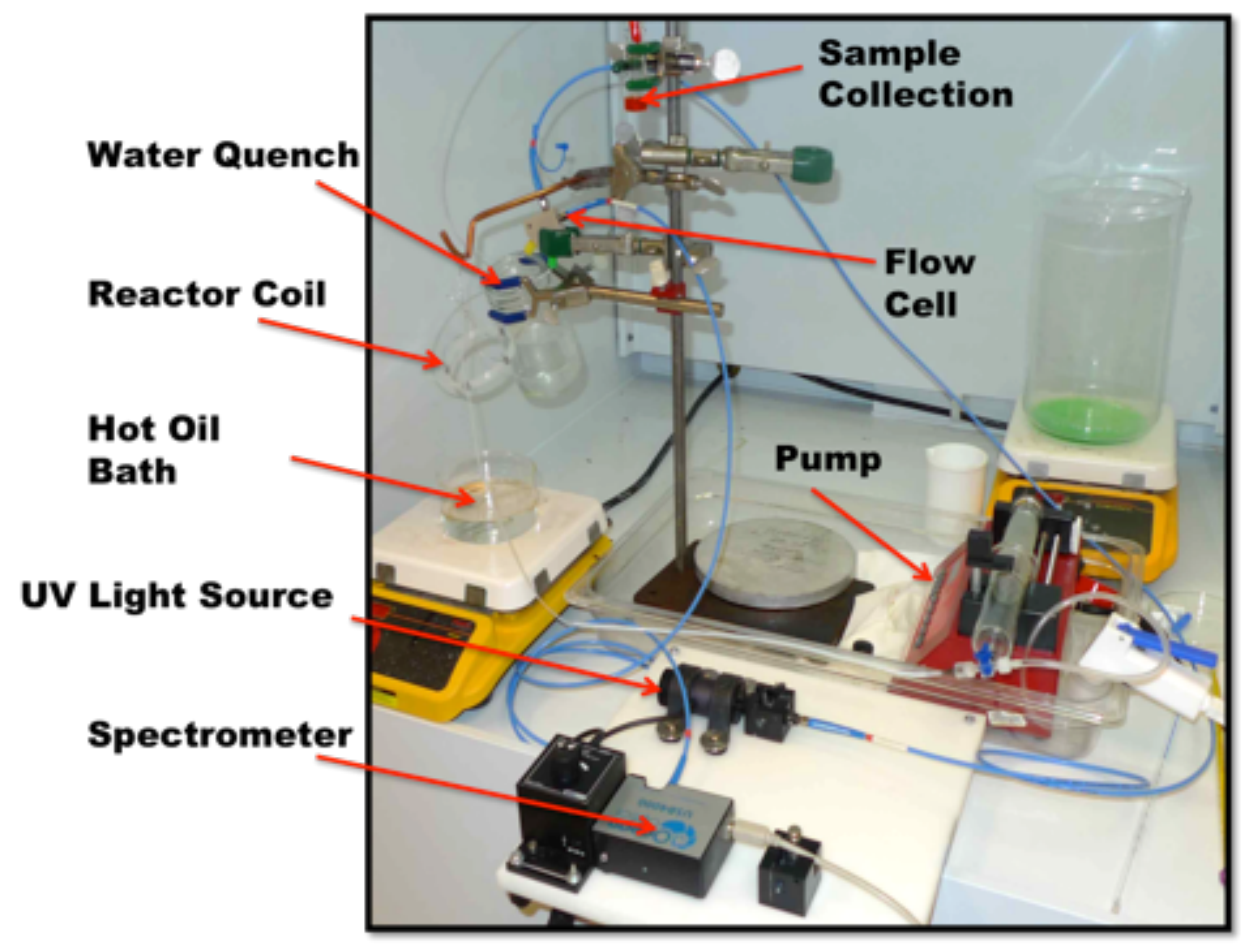

Figure 10: Photo of the test setup for synthesizing quantum dots

The quantum dot solution was then collected in various glass vials for analysis. The entire process enables a relatively quick synthesis and characterization, while being confined in a small area within the fume hood. Proper clean room safety equipment and 
procedures were used whenever dealing with the experimental test fixtures; protective equipment included gloves, booties, caps, safety glasses, and lab coats.

\subsection{Experimental Procedure}

In preparation to synthesize quantum dots, separate cadmium and selenium precursor solutions were synthesized in octadecene following standard procedures outlined in the Cal Poly QD SOP. ${ }^{43}$

\subsubsection{Microfluidic Synthesis Procedure}

Prior to beginning synthesis, all equipment was organized and proper safety precautions were followed. First, a water bath was prepared for mixing the two precursor solutions. A $1000 \mathrm{ml}$ beaker was filled half full with cool tap water and a stir bar was added. A beaker was placed on a cool hotplate and the stir feature was enabled. Ice cubes were added to chill the water to $17^{\circ} \mathrm{C}$. A glass thermometer was used to control the temperature and prevent freezing of octadecene. The individual cadmium and selenium precursor solutions were then chilled at $17^{\circ} \mathrm{C}$ for 10 minutes. The selenium precursor solution, once thoroughly cooled, was extracted using the syringe process outlined in the Cal Poly QD SOP ${ }^{43}$ and combined with the cadmium precursor solution. The reagent mixture was then returned to the chilled water bath and allowed to mix at $17^{\circ} \mathrm{C}$ for 5 minutes. A 50cc syringe was cleaned thoroughly with acetone and dried with the nitrogen gun in the clean room. It was then placed in the clean room refrigerator until the precursor solutions were fully mixed. Then the pressure of the nitrogen system in the clean room was lowered to $4 \mathrm{psi}$ to prevent pressure blowout. The 50cc syringe was 
lubricated with octadecene to prevent both locking during synthesis and air contamination during extraction. Once mixed $55 \mathrm{ml}$ of the reagent mixture was extracted from the $100 \mathrm{ml}$ three-neck round bottom flask and transferred into the 50cc syringe using the syringing process described in the Cal Poly QD SOP ${ }^{43}$. The syringe needle was immediately removed and the syringe was attached to the three-way valve by twisting. Oxygen contamination was minimized by expelling $5 \mathrm{ml}$ of the reagent solution while making the connection to the valve. The syringe was then loaded into the syringe pump and the diameter setting on the syringe pump was set to $27.94 \mathrm{~mm}$ to assure proper flow rates. Flow rates were chosen initially by calculating desired residence times using the bulk synthesis process as a reference, then extrapolating to the microfluidic process. Sixteen testable flow rates were selected for characterization (Table III).

Table III: Selected flow rates to be analyzed in all subsequent trials

\begin{tabular}{|l|l|l|l|l|l|l|l|l|}
\hline $\begin{array}{l}\text { Flow Rate } \\
(\mathrm{ml} / \mathrm{min})\end{array}$ & 0.08 & 0.1 & 0.2 & 0.3 & 0.4 & 0.5 & 0.6 & 0.7 \\
\cline { 2 - 9 } & 0.8 & 0.9 & 1 & 1.1 & 1.2 & 1.3 & 1.4 & 1.5 \\
\hline
\end{tabular}

The syringe containing the reagent mixture was kept at $17^{\circ} \mathrm{C}$ for the duration of the synthesis by periodically dipping a cloth into an ice bath and then wrapping it around the syringe to minimize QD formation at room temperature. The reactor coil was then lowered into the hot oil bath pre-heated to $235^{\circ} \mathrm{C}$ and the entire system was purged with nitrogen for one minute. Using the three-way valve the nitrogen line was switched off and the syringe pump pathway was switched open. After the reaction came to equilibrium and the fluorescence spectrum stabilized, data were recorded. The system was purged of 
reactive solution using nitrogen gas between each flow rate trial and samples were collected in separate vials.

\subsection{Data Acquisition Procedure}

In order to accurately measure the fluorescence profiles of quantum dots, a ThorLabs ${ }^{\circledR}$ LED UV light source was connected by a fiber optic cable to the flow cell. Light at $385 \mathrm{~nm}$ was emitted and carried by fiber optic cable into the quantum dot solution within the flow cell. A second fiber optic cable connected to the flow cell collected the fluorescence spectrum of the quantum dots and sent it into an Ocean Optics ${ }^{\circledR}$ USB4000 Spectrometer. This spectrometer transmits data to the software SpectraSuite ${ }^{\circledR}$.

\subsubsection{SpectraSuite ${ }^{\circledR}$ Software}

SpectraSuite $^{\circledR}$ is a modular, Java-based spectroscopy software platform that operates on 32- and 64-bit Windows, Macintosh and Linux operating systems. ${ }^{44}$ The software can control any Ocean Optics ${ }^{\circledR}$ USB spectrometer and device. Because this experiment relied on fluorescence in the visible spectrum, the analysis technique was set to the "Scope" setting. In order to calibrate the Spectrometer, a dark spectrum was acquired by turning the light source off. A light spectrum was also captured to determine spectrum of the UV light source. Software parameters such as integration time, scans to average, and boxcar width affect data acquisition and are listed (Figure 11). "Integration time" accounts for how long the spectrometer will capture the incoming photons. This variable is synonymous to a camera shutter. "Scans to average" allows multiple scans to be averaged 
over time. "Boxcar width" takes the spectral data and averages nearby data points to reduce the background noise of the spectrometer.

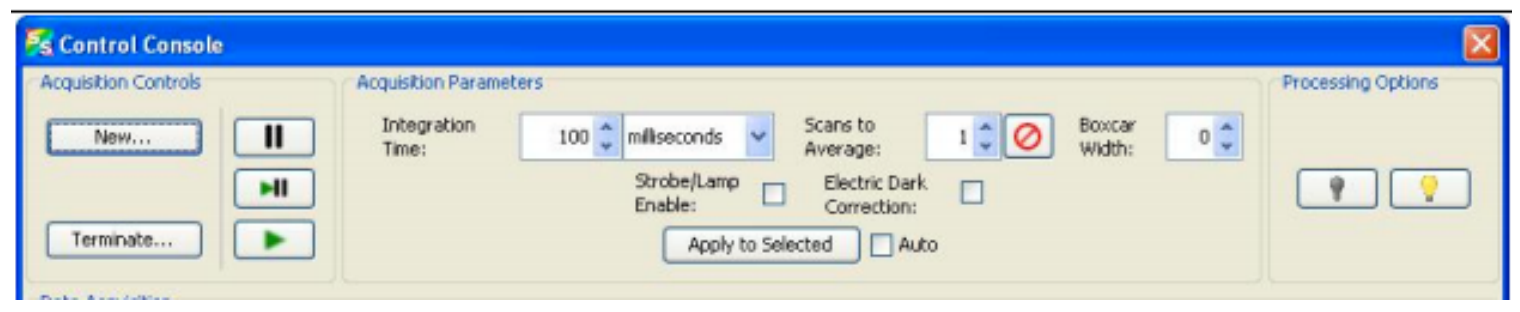

Figure 11: Acquisition control panel depicting integration time, boxcar width, and scans to average (center). Dark and light spectrums (right) are used to calibrate the spectrometer.

For this experiment, the integration time was set to 500 milliseconds to allow enough photon counts to register a distinct peak wavelength. Because this process used segmented continuous flows, there is not much variance between samples, so the "scans to average" was only set to 2 . Due to a presence of noise within the spectrometer, the boxcar width was set to 5 to minimize the effects of noise. SpectraSuite ${ }^{\circledR}$ enables continuous data capturing as well as manual modes of capturing by clicking the mouse. A manual mode was employed to capture each spectral profile by clicking the mouse for each trial. A total of 15 fluorescence profiles were collected for each flow rate and then transferred into Excel spreadsheets for data analysis and repeatability results.

\subsection{Design Constraints}

Empirical testing indicates that this experimental setup can produce quantum dots between $490 \mathrm{~nm}$ to $590 \mathrm{~nm}$. Researchers have found that the growth reaction of Cd-Se quantum dots comes to equilibrium when the dots are about $4.1 \mathrm{~nm}$ in size, which is comparable to a fluorescence profile of $590 \mathrm{~nm}$. To get quantum dot sizes up to $5.5 \mathrm{~nm}$, 
more precursor solution must be added to the original quantum dots. The original quantum dots can be essentially "re-grown" with the excess precursor solution when

reacted at a lower synthesis temperature. ${ }^{49}$ Maximum synthesis temperature for the $\mathrm{TEFLON}^{\circledR}$ reactor was limited by the maximum service temperature, $271^{\circ} \mathrm{C}$. Using a higher synthesis temperature in a microfluidic system would facilitate more precise control of nucleation and growth effects within the system, as the initial nucleation burst would transpire more quickly leaving more time for focusing growth. ${ }^{27}$

\subsection{Proof of Concept Reactor Results}

\subsubsection{Data Manipulation}

Initial raw data from SpectraSuite ${ }^{\circledR}$ software were analyzed using Microsoft Excel and Minitab. Due to noise in the raw data, manipulation was required.

\subsubsection{Moving-Median Smoothing (P.O.C Reactor)}

Fifteen fluorescence profiles were recorded for each flow rate. The data were then averaged to obtain a raw mean spectral profile shown in blue (Figure 12). These raw data were smoothed utilizing a moving-median smoothing macro in Minitab. The purple line shown on Figure 12 represents the results of the smoothing. A Gauss-Newton regression was then applied using the Bragg equation in Minitab to generate lines of best fit (shown in red) and determine the center wavelength of the peaks. 


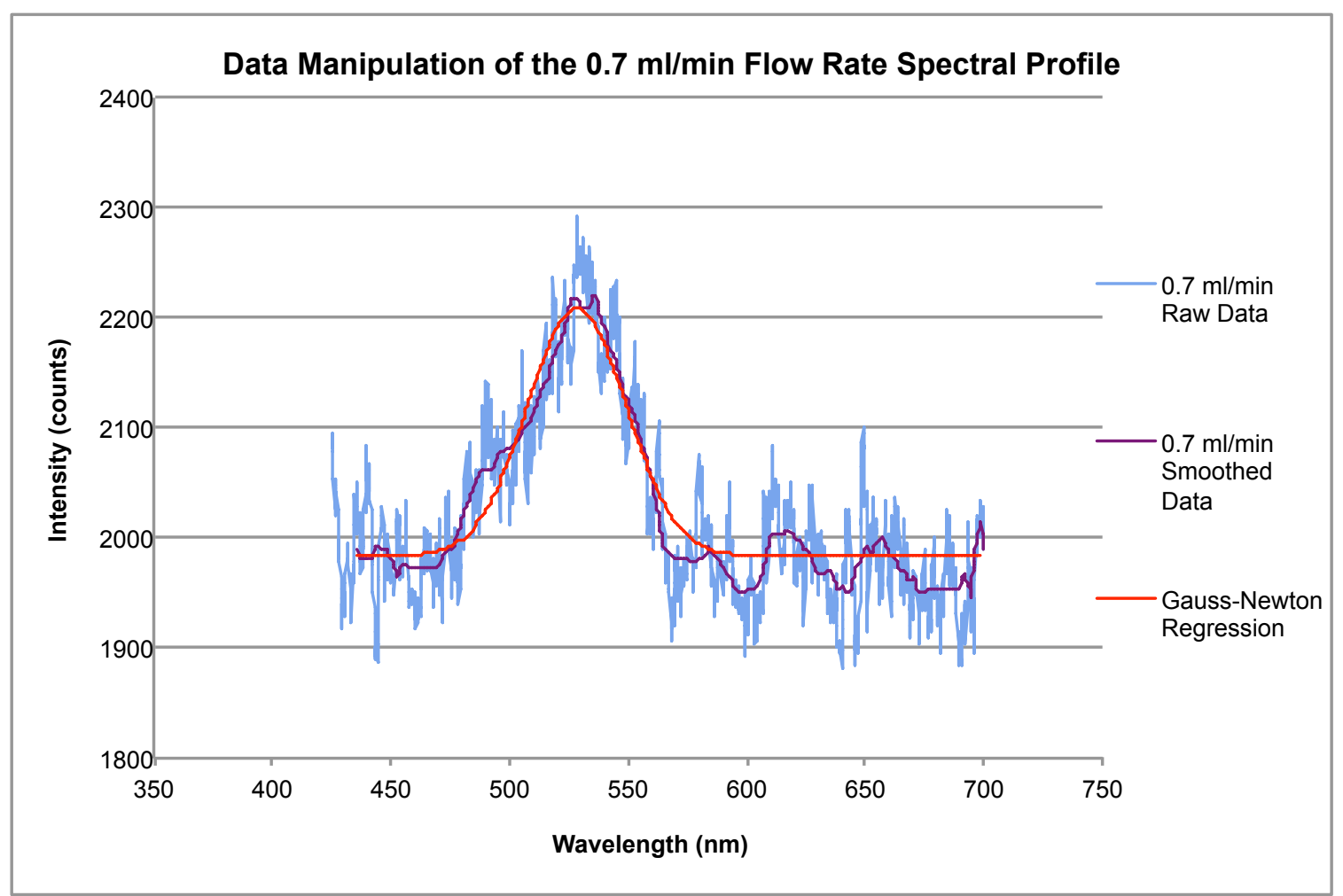

Figure 12: Example of data manipulation applied to all flow rates to obtain best-fit curves for spectral peaks and relative center wavelength.

Gauss-Newton regressions were performed on all flow rates for the collective trials. The resulting curves were then compared between subsequent trials. Six flow rates were selected to demonstrate the shift in center wavelength due to flow rate and the relative intensities of the peaks (Figure 13). 


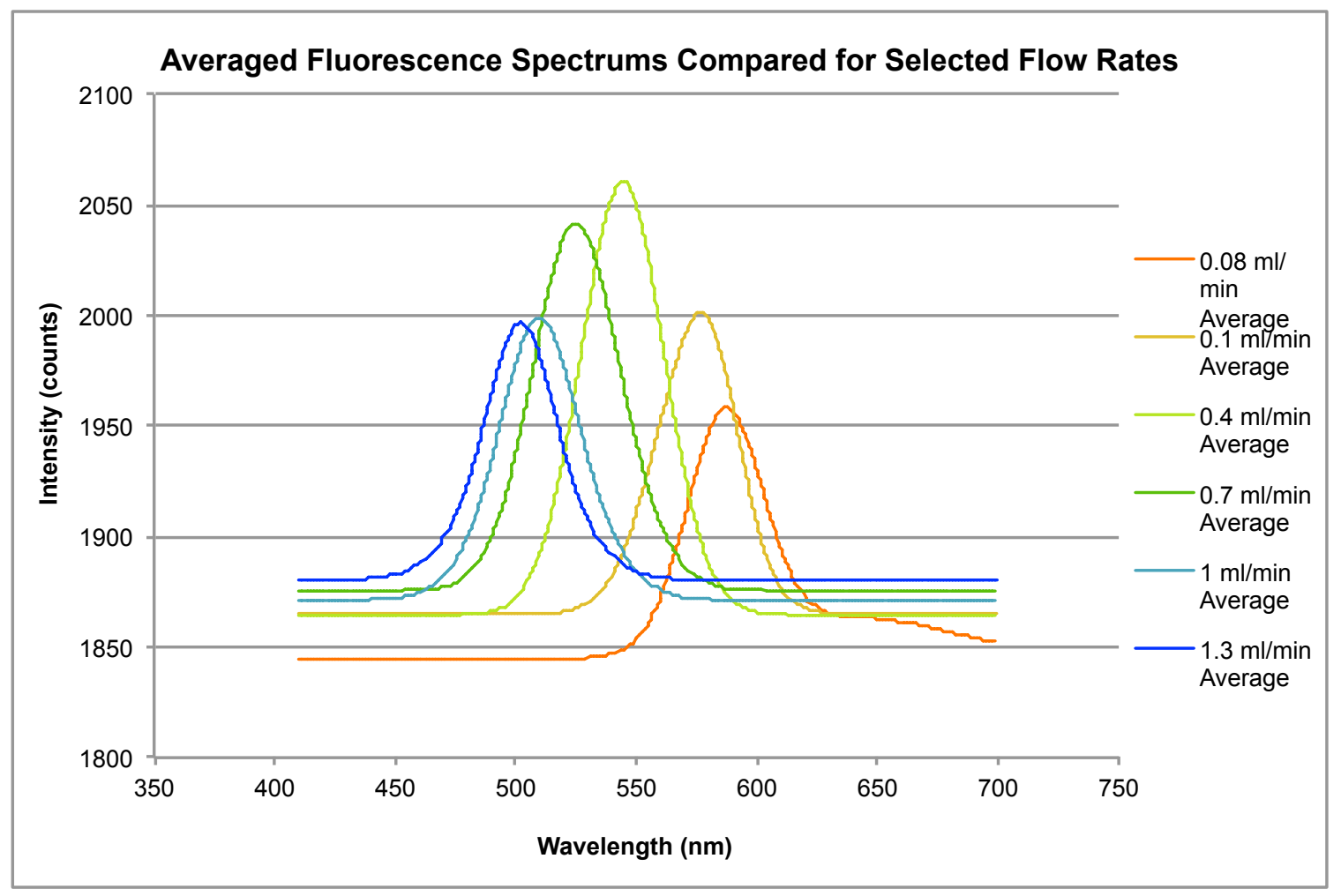

Figure 13: Average of three trials Gauss Newton regressions at six selected flow rates. Note the left shift of the peaks with increasing flow rate.

The center wavelength shifts left as the flow rate increases. Additionally, the fluorescent intensity decreases as the wavelength increases and the quantum dots grow. Defects in the Cd-Se core as well as on the surface act as energy sinks, lowering the intensity of light emitted from quantum dots during fluorescence. ${ }^{45}$ This could describe the lower intensities of higher wavelength quantum dots as they are larger and have more surface area for defects to form.

\subsubsection{Relationship of Flow Rate to Peak Center Wavelength}

The center wavelengths from the collective trials were arranged by flow rate and averaged to obtain the mean center wavelength for each flow rate. Then the standard 
deviation for each flow rate was calculated and applied to the error bars shown on Figure 14.

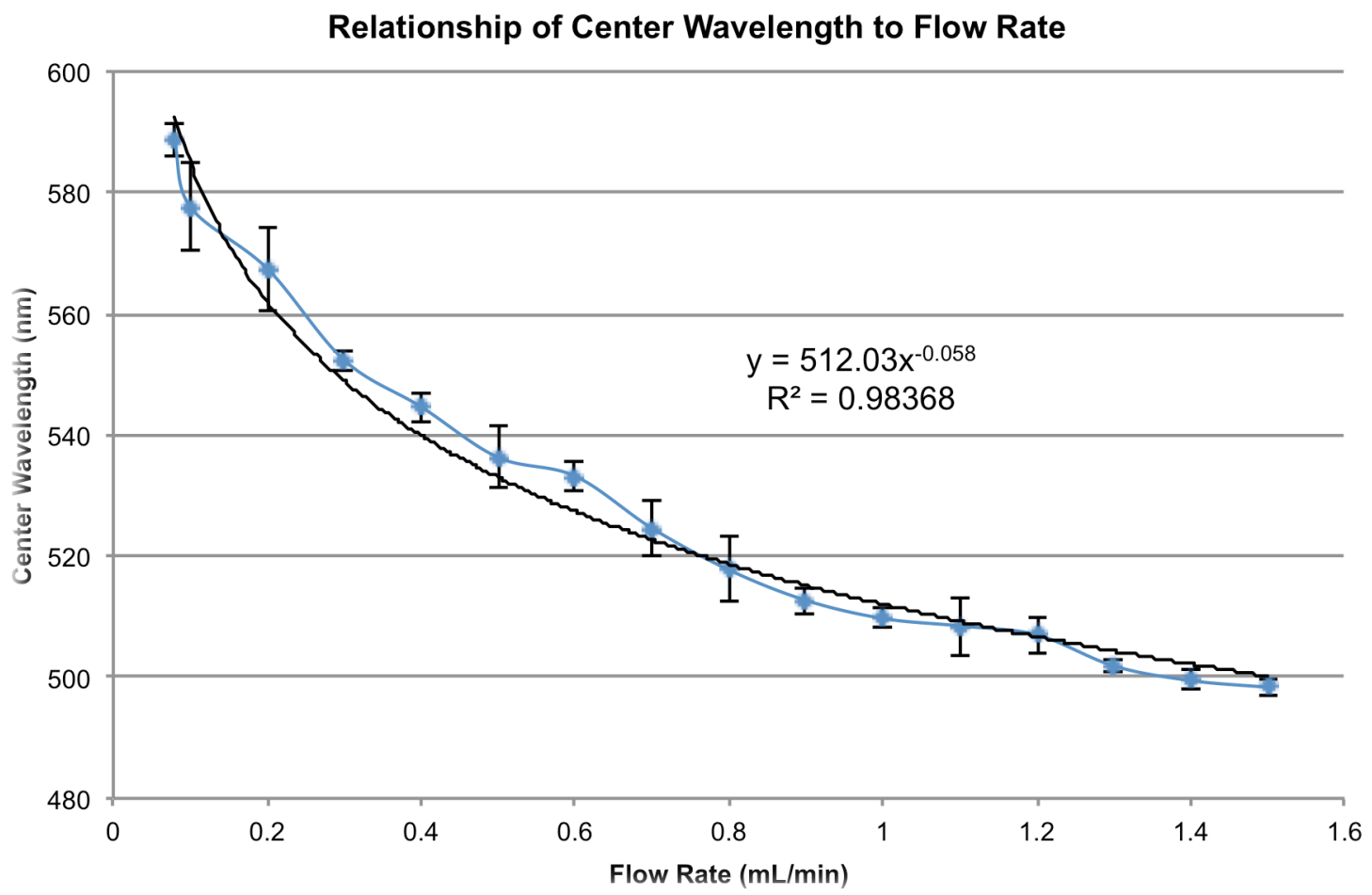

Figure 14: A power function regression was used to derive an empirical equation relating flow rate to center wavelength. The error bars represent the standard deviation between all collective trials at the specified flow rates.

A line of best fit was calculated to determine whether a relationship exists between flow rate and center wavelength. The equation of the trend line will allow for precise control of wavelength and size of quantum dots utilizing flow rate as the primary variable (Equation 8).

Equation 8: Power function relationship between flow rate and center wavelength

$$
\lambda_{\begin{array}{c}
\text { Average Center } \\
\text { Wavelength }(\mathrm{nm})
\end{array}}=512.03\left(Q_{\begin{array}{l}
\text { Volumetric } \\
\text { Flow Rate }(\mathrm{ml} / \mathrm{min})
\end{array}}\right)^{-0.058}
$$


The center wavelength of the peaks followed a power function with an $\mathrm{R}^{2}$ value of 0.98 .

\subsubsection{Analysis of Particle Diameter}

An empirical equation developed at the University of Arkansas was used to calculate the average particle diameter from the center wavelength for each flow rate (Equation 9). ${ }^{46}$

Equation 9: Empirical equation relating center wavelength to particle diameter

$$
\begin{aligned}
& \mathrm{D}=\left(1.6122 \mathrm{E}^{-9}\right) \lambda^{4}-\left(2.6575 \mathrm{E}^{-6}\right) \lambda^{3}+\left(1.6242 \mathrm{E}^{-3}\right) \lambda^{2}-\left(4.277 \mathrm{E}^{-1}\right) \lambda+(41.57) \\
& \lambda=\text { Center wavelength of peaks }(\mathrm{nm}) \quad D=\text { Avg particle diameter }(\mathrm{nm})
\end{aligned}
$$

The average particle diameter for each tested flow rate ranged from $2.2 \mathrm{~nm}$ to $4.1 \mathrm{~nm}$ (Figure 15). Proof Of Concept Reactor Relationship of Average Particle Diameter to
Flow Rate

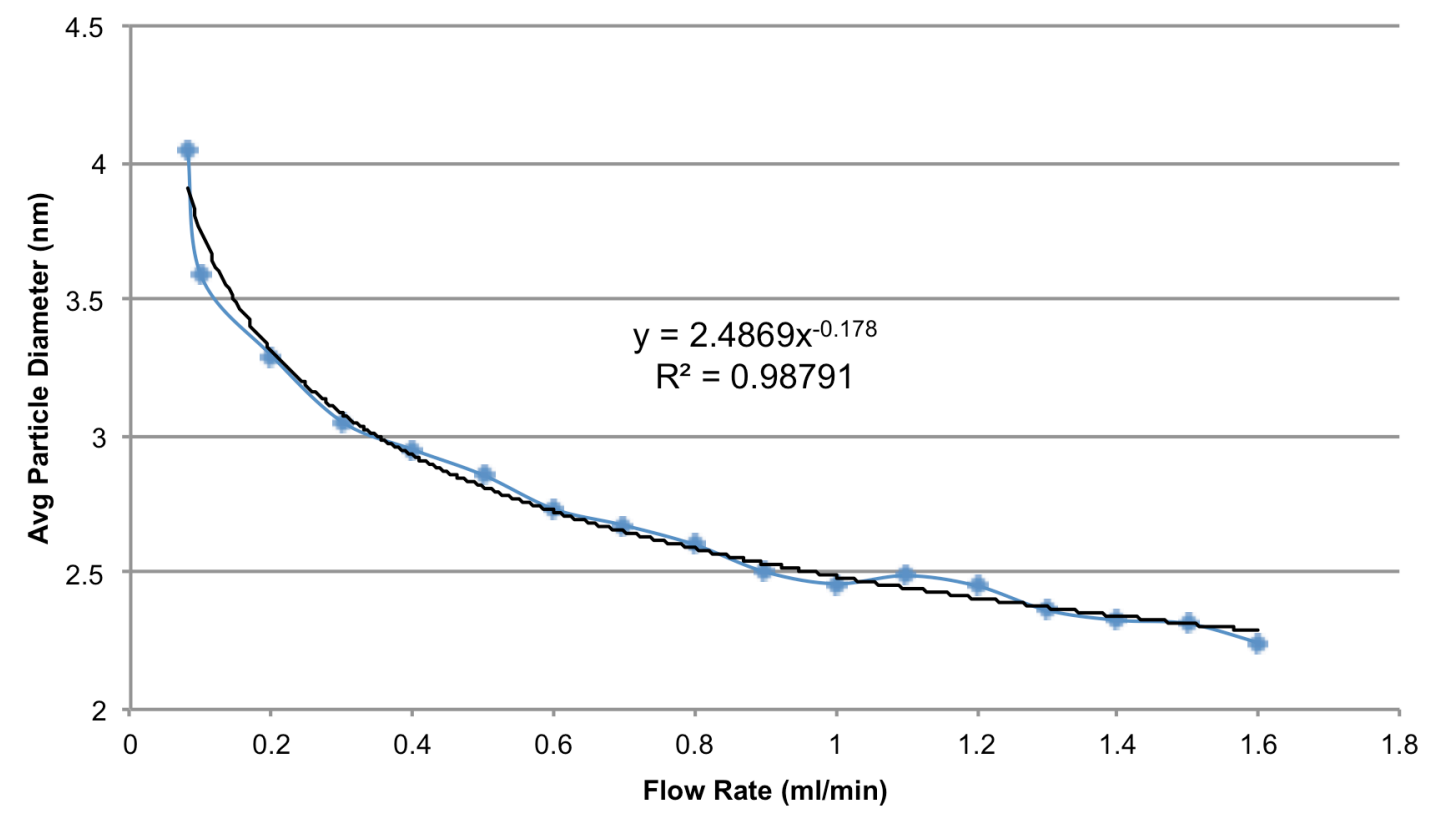

Figure 15: Average particle diameter for each tested flow rate (Proof of Concept Reactor). 
Particle diameter increased as flow rate decreased because particles had more time at the reaction temperature to grow.

\subsubsection{Repeatability}

The standard deviation between all experimental trials was calculated for the assessed flow rates (Table IV). The average standard deviation was $3 \mathrm{~nm}$. Because the standard deviations are mostly within $5 \mathrm{~nm}$, this synthesis process exhibits a high degree of accuracy for producing desired central wavelengths.

Table IV: Calculated standard deviation of center wavelength at tested flow rates.

\begin{tabular}{|c|c|}
\hline $\begin{array}{c}\text { Flow Rate } \\
(\mathbf{m l} / \mathbf{m i n})\end{array}$ & $\begin{array}{c}\text { Standard Deviation of Center } \\
\text { Wavelength } \mathbf{( n m})\end{array}$ \\
\hline 0.08 & 2 \\
\hline 0.1 & 7 \\
\hline 0.2 & 7 \\
\hline 0.3 & 2 \\
\hline 0.4 & 2 \\
\hline 0.5 & 5 \\
\hline 0.6 & 3 \\
\hline 0.7 & 5 \\
\hline 0.8 & 5 \\
\hline 0.9 & 2 \\
\hline 1.0 & 2 \\
\hline 1.1 & 5 \\
\hline 1.2 & 3 \\
\hline 1.3 & 1 \\
\hline 1.4 & 2 \\
\hline 1.5 & 2 \\
\hline Average StDev & 3 \\
\hline
\end{tabular}

The spectrometer is accurate to $1 \mathrm{~nm}$; so most of the standard deviations shown above are likely due to human error and difficulties keeping the oil bath at $235^{\circ} \mathrm{C}$. 


\section{PROOF OF CONCEPT REACTOR OPTIMIZATION}

Design modifications were made to the Proof of Concept Reactor with the intention of improving process reliability, accuracy, and ease of use. Optimization steps and results are summarized below.

\subsection{Light Source and Light Source Fixture Modification}

A new light source (THOR Labs, 385nm, 700mA) was used to decouple the reactor test setup from a cuvette spectrometry-testing platform. When using the cuvette spectrometry-testing platform the distance between the LED light source and the fiber optic cable resulted in low transmission of light through the fiber optic cable (Figure 16a). As distance between the light source and the fiber optic cable increases the emitted beam becomes more disperse. Without a lens to focus the light on the fiber optic cable the majority of light created by the LED was not transmitted to the fiber optic cable. An alignment chuck was used to position the fiber optic cable as close as possible to the LED, drastically increasing the flux through the fiber optic cable (Figure 16b).

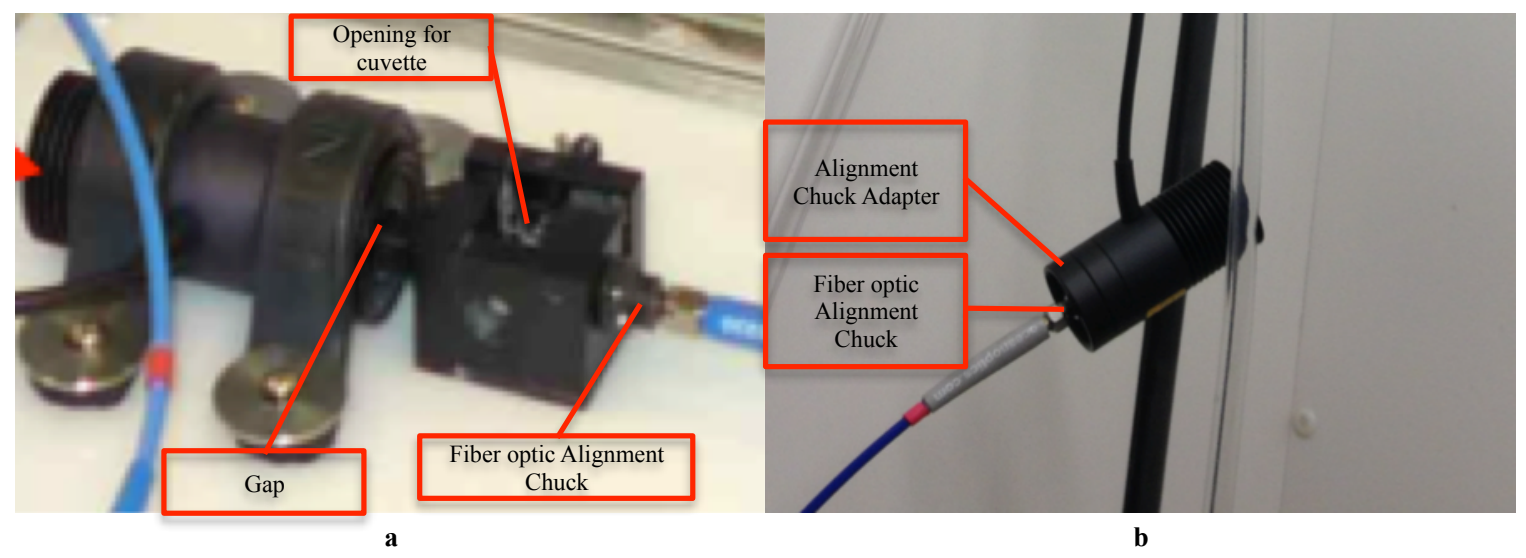

Figure 16: a) Preliminary light source setup with a LED attached to a cuvette chuck. b) New light source with an alignment chuck and adapter. 
With this modification higher intensity peaks were registered in the flow cell, eliminating the need for post processing of the SpectraSuite ${ }^{\circledR}$ fluorescence data with a median smoothing algorithm.

\subsection{Modified Precursor Cooling Method}

In the Proof of Concept Reactor a piece of cloth dipped in ice water was used to chill the precursor solution. There was not enough time to continuously dip the rag in an ice water bath and record data simultaneously during a trial. This method introduced a large margin for human error. Keeping the precursor solution at a constant temperature was neglected to take data. Additionally, water from the rag dripped all over the fume hood creating a messy working environment.

\subsubsection{Ethylene Glycol Chiller Unit Construction}

An ethylene glycol based chiller unit was modified to replace the wet cloth (Figure 17 a). 


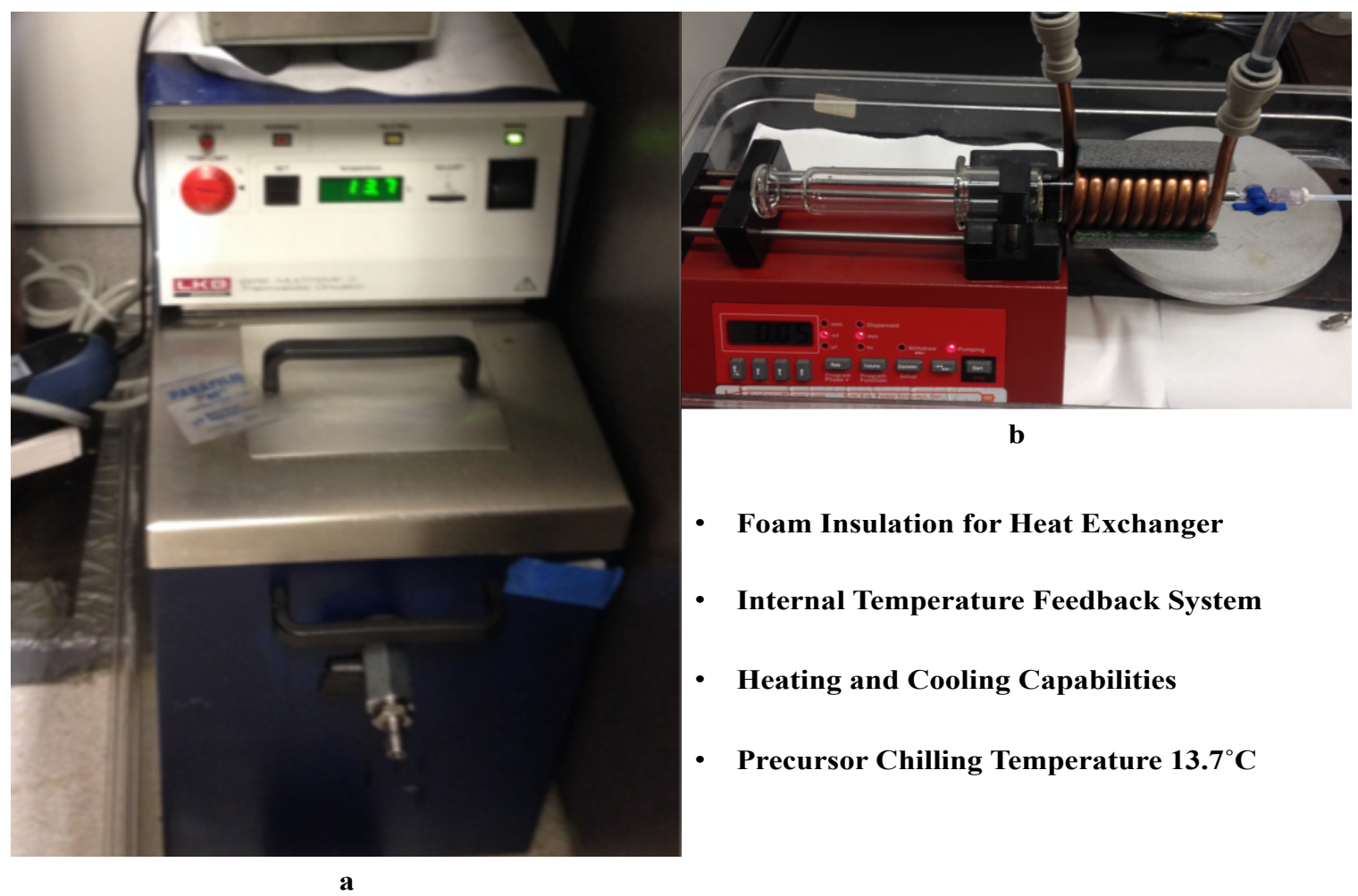

Figure 17: a) Ethylene glycol chiller system. b) Copper heat exchanger coil wrapped around glass 50ml syringe

A heat exchanger was constructed from quarter inch copper refrigerator tube bent in a coil around a $50 \mathrm{ml}$ syringe. The coil was cut to length to fit over the syringe while it was suspended on the syringe pump (Figure $17 \mathrm{~b}$ ). This coil was attached to half inch Tygon tubing with press fit adapters.

A temperature range from $25^{\circ} \mathrm{C}$ to $12^{\circ} \mathrm{C}$ was tested to determine the lowest temperature the coil could be held without freezing the octadecene solution. Freezing of the octadecene solution in the syringe results in seizing during pumping. A temperature of $13.7^{\circ} \mathrm{C}$ was selected and the coil was insulated with standard 2-inch pipe insulation to maximize thermal transfer to the heat exchanger. 


\subsubsection{Ethylene Glycol Chiller Testing and Results}

First the LED light source and spectrometer were turned on for two hours prior to testing to ensure equilibrium of the diode and detector. A baseline spectral profile was recorded using a cuvette measurement system immediately following mixing of a small amount of precursor solutions. The precursor solution was then split into two $50 \mathrm{ml}$ glass syringes equally. One syringe of the mixed solution was attached to the chiller and left for four hours. The other syringe was left at room temperature for four hours. Measurements for both syringes were recorded using cuvette spectral analysis every two hours (Figure 18).

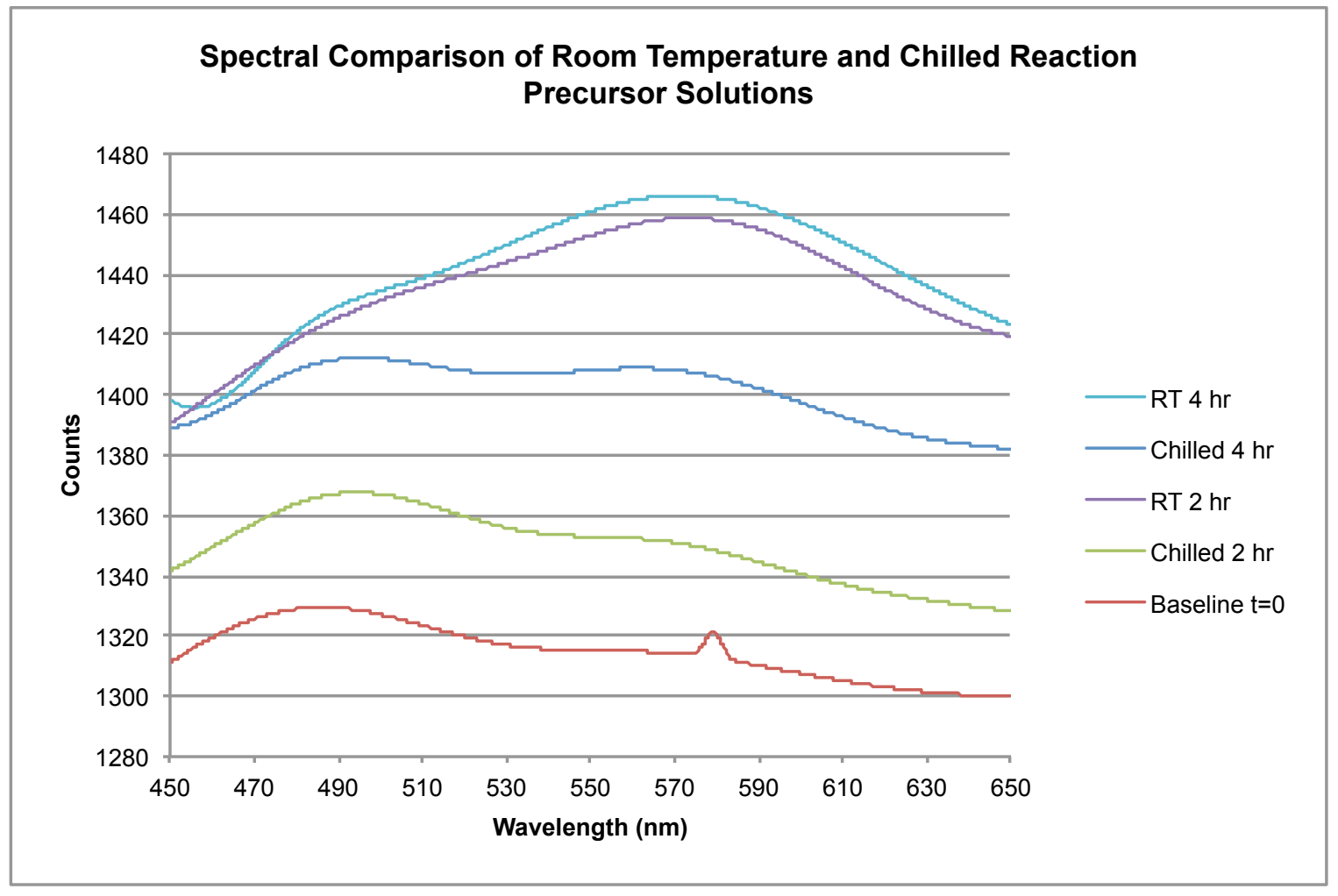

Figure 18: Nucleation and growth of chilled quantum dots and room temperature quantum dots over a time frame of four hours.

The initial sample (Red) was used as a baseline $(\mathrm{t}=0)$ to compare the relative extent of nucleation and growth. A peak formed in the $2 \mathrm{hr}$ room temperature sample reaching an 
intensity of 1460 counts. After four hours this peak had increased to 1465 counts. The $2 \mathrm{hr}$ room temperature sample had a maximum intensity of 1365 and the $4 \mathrm{hr}$ chilled sample reached a maximum intensity of 1410 .

\subsubsection{Ethylene Glycol Chiller Discussion}

To understand the benefits of chilling the reagent solution, one must first understand the progression of the nucleation and growth reaction and how kinetics and thermodynamics relate to the reaction as discussed in Chapter 2. When the cadmium precursor solution and the selenium precursor solution are mixed at a temperature of $17^{\circ} \mathrm{C}$ the reaction monomers do not fully dissociate in solution. Temperature controls the degree of dissolution and temperatures in excess of $200^{\circ} \mathrm{C}$ are needed to fully break down the precursors into a supersaturated solution of reaction monomers. By lowering the temperature of the two precursor solutions and keeping them low until they flow through the reactor the amount of nucleation and growth can be limited because the concentration of reaction monomers in solution is depressed. With fewer reaction monomers in solution a large degree of nucleation cannot occur. Despite this, is important to note that reducing the temperature of the solution increases the degree of undercooling. Increasing the degree of undercooling in a phase transformation translates directly to an increase in the driving force of the phase transformation while simultaneously lowering the critical radius. Nucleates are thermodynamically preferred to a supersaturated solution in a twophase system with a large degree of undercooling. Growth of these nucleates, however, relies on diffusion, which is thermally activated. Nucleation and growth occur most rapidly when a balance between the driving force for nucleation (the degree of 
undercooling) and the kinetic aspect of growth (thermally activated diffusion) is achieved. ${ }^{47}$ Conversely, by limiting the amount of thermal energy of the system using a chiller, the diffusion process can be slowed and the growth of quantum dots can be effectively constrained as diffusion is inhibited. Additionally, activation energy is required for reaction monomers to be incorporated in the quantum dot lattice. Lowering the temperature of the overall system also lowers the rate of incorporation. All of these phenomena contribute to the benefits of the chiller system shown on Figure 18. The fluorescent profile for the $2 \mathrm{hr}$-chilled sample suggests significantly less nucleation and growth occurs compared to the $2 \mathrm{hr}$-aged room temperature sample. Less disparity exists between the $4 \mathrm{hr}$-aged samples. Despite limiting the diffusion, given enough time, quantum dots will still nucleate and grow due to the high degree of undercooling. Synthesis of $50 \mathrm{ml}$ of Cd-Se quantum dots takes on average about $4 \mathrm{hrs}$. This is a significant amount of time for the nucleation and growth reaction to occur, however, chilling the precursor solution for the duration of the test is still beneficial when comparing the degree of nucleation and growth for the four hour chilled and room temperature samples. A better method of minimizing room temperature nucleation and growth would potentially be to mix the two precursor solutions in a microfluidic mixer prior to injection into the reactor. This will be further described in the future work section of this thesis.

\subsection{Optimized Experimental Procedure}

The experimental procedure from the Proof of Concept Reactor and the Cal Poly QD SOP were optimized for use in the final reactor system. ${ }^{43}$ All processes outlined in this 
section were used to characterize the reactor system. Before initiating the experimental procedure the reactor system was inspected for clogs and leaks, fiber optic cables were attached, and the USB spectrometer was plugged into a computer.

\subsubsection{Syringing Process}

The syringing process is a method developed to minimize contamination that arises during the transfer and combination of reactant chemicals. This process will be referred to as the "syringing process" in all instances of use. Before transferring any chemicals the initial container, end receptacle, and transfer syringe are purged of oxygen. This was accomplished by inserting a nitrogen line needle and a vent needle through a rubber septum and flushing out oxygen with an inert gas (nitrogen was used in this study). First the vent needle was removed from the fully purged initial container creating positive pressure. Then the syringe needle was inserted through the rubber septum into the nitrogen on the other side. Nitrogen from the chemical container was drawn into the syringe and expelled to atmosphere three times to purge any oxygen trapped inside. The solution was then drawn and transferred to the end receptacle.

\subsubsection{Precursor Synthesis Method}

First one $100 \mathrm{ml}$ three-neck flask and one two-neck $50 \mathrm{ml}$ flask were thoroughly cleaned with acetone and dried. A stir bar was then added to each flask. $33 \mathrm{mg}$ selenium powder was measured onto a scoopula resting on an accurate scale. This powder was then transferred into the $50 \mathrm{ml}$ two-neck flask and both necks were capped with rubber septum. $65 \mathrm{mg}$ cadmium oxide was then measured and transferred to the $100 \mathrm{ml}$ three-neck flask 
using the same procedure. The flasks were kept upright using small dishes to ensure all powder precursor remained at the bottom of the flask to ensure a complete reaction. The selenium precursor in the $50 \mathrm{ml}$ two-neck flask was suspended over a high temperature silicon oil reaction bath heated to $150^{\circ} \mathrm{C}$ with a stir speed of 500rpm. The flask was purged of oxygen using a nitrogen needle and vent needle for 10 minutes. Octadecene and trioctylphosphine the liquid components for the selenium precursor were purged for $10 \mathrm{~min}$ as well. Using a $10 \mathrm{ml}$ syringe $10 \mathrm{ml}$ octadecene was drawn using the syringing process described above. $5 \mathrm{ml}$ of the $10 \mathrm{ml}$ of octadecene drawn into the syringe was transferred to the selenium two-neck reaction flask. The remaining $5 \mathrm{ml}$ was later used to lubricate the $50 \mathrm{ml}$ glass syringe and to purge the microfluidic line of oxygen before synthesis. Once the octadecene has come to reaction temperature (about $5 \mathrm{~min}$ ) $0.4 \mathrm{ml}$ trioctylphosphine was added to the selenium two-neck reaction flask using the syringing process. This solution was then left for 10-20 minutes until all the black selenium powder dissolved. The selenium two-neck reaction flask was continually purged with nitrogen for the duration. Once fully reacted the reaction flask was removed from the heating bath and placed upright to the side to cool. The vent needle was removed but the nitrogen needle was left in to prevent any oxygen from contaminating the solution. The high temperature silicon oil bath was then raised to $265^{\circ} \mathrm{C}$ with a stir speed of 500rpm. The cadmium oxide three-neck reaction flask was suspended in the reaction bath and purged for 10 min using the same process as the selenium precursor solution. Once purged, $50 \mathrm{ml}$ of purged octadecene was transferred into the cadmium oxide three-neck reaction flask using the syringing process. After the reaction flask reached temperature (about $15 \mathrm{~min}), 3 \mathrm{ml}$ of purged oleic acid was added using the syringing process. After the addition of the oleic 
acid the cadmium precursor solution changes color from a dull red to clear (about 30 minutes). The flask was purged the entire time to allow removal of oxygen as the cadmium oxide reacts. The cadmium oxide three-neck reaction flask was then removed from the silicon oil bath and allowed to cool. For the duration of cooling the vent needle was removed to create positive pressure in the cadmium precursor flask.

\subsubsection{Precursor Solution Mixing Procedure}

A large beaker was filled with chilled water at $14^{\circ} \mathrm{C}$ and a stir bar was added for mixing. The beaker was placed on a cool hotplate with the stir function enabled at 500rpm. Both precursor flasks were placed in the chilled water bath and adjusted so the cadmium precursor stir bar mixed the solution (Figure 19).

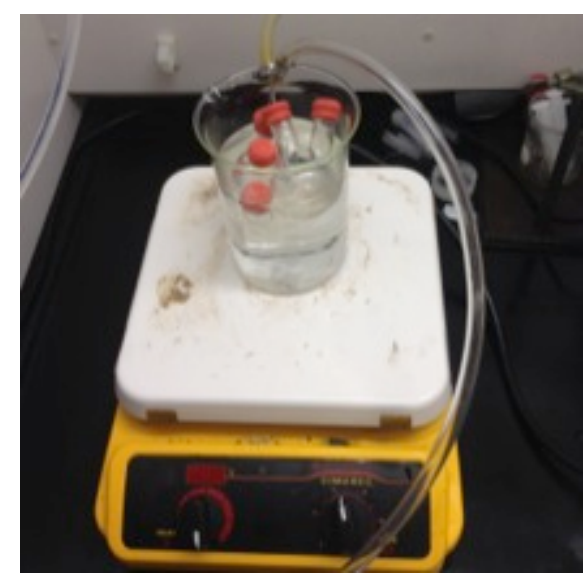

Figure 19: Precursor solution mixing setup

When the solution becomes sufficiently chilled the cadmium precursor solution becomes slightly slushy and was removed from the chilled water bath. The selenium precursor was then added using the syringing process. The combined precursor solutions were then mixed for two minutes. While mixing a 50ml syringe was prepped. A two-way leur lock 
valve was attached to the syringe and a needle was attached to the valve in line with the syringe. The syringe piston was lubricated with a small amount of the octadecene left over in the $10 \mathrm{ml}$ syringe. Next the syringe was chilled using the copper coil chiller attachment. The chilled solution was then transferred, using the syringing process, to the chilled $50 \mathrm{ml}$ glass syringe. The remaining octadecene in the $10 \mathrm{ml}$ syringe from earlier was then dispensed into the reaction coil using hand pressure to purge oxygen from the tubing. After the reaction coil became completely filled with octadecene the $50 \mathrm{ml}$ syringe was attached. A flow rate of $1 \mathrm{ml} / \mathrm{min}$ was selected on the syringe pump to dispense for 3 min to completely flush the reactor of pure octadecene.

\subsubsection{Reactor Characterization Procedure}

The flow rate was then set to $0.05 \mathrm{ml} / \mathrm{min}$ and allowed to come to equilibrium (about 30 minutes). Flow rate was then increased and allowed to come to equilibrium for each subsequent flow rate. An equation was created to relate flow rate to time until equilibrium is reached (Equation 10).

Equation 10: Time required to reach equilibrium at a given flow rate.

$$
\left(\text { Time }_{(\min )}\right)=(2)\left(\text { Flowrate }_{(\mathrm{ml} / \mathrm{min})}\right)
$$

SpectraSuite ${ }^{\circledR}$ software was used to record data for each flowrate. For the sake of management Table $\mathrm{V}$ lists the flow rates tested in the optimized reactor.

Table V: Selected flow rates to be analyzed in all subsequent P.O.C reactor trials

\begin{tabular}{|c|c|c|c|c|c|c|c|c|c|c|}
\hline $\begin{array}{c}\text { Flow Rate } \\
(\mathrm{ml} / \mathrm{min})\end{array}$ & 0.05 & 0.07 & 0.09 & 0.1 & 0.15 & 0.2 & 0.3 & 0.4 & 0.5 & 0.6 \\
\cline { 2 - 11 } & 0.7 & 0.8 & 0.9 & 1 & 1.2 & 1.4 & 1.6 & 1.8 & 2 & \\
\hline
\end{tabular}




\subsubsection{Optimized SpectraSuite ${ }^{\circledR}$ Data Acquisition Procedure}

Initially SpectraSuite ${ }^{\circledR}$ software was only functional on Windows XP computers and could not be installed on computers running windows 7 or OS-X. This posed a problem because the processing power of the available laptops could not handle increasing the "scans to average" or "boxcar width" to obtain a clean signal. The noisy spectral data obtained with this set up required manipulation. To obtain a reasonable peak a non realtime median smoothing algorithm in Minitab had to be used to smooth the data. This was time consuming and made statistical characterization of the peaks less accurate. To remedy this problem the most current version of SpectraSuite ${ }^{\circledR}$ was obtained by speaking to a service representative for Ocean Optics ${ }^{\circledR}$ who provided a new product license code. Once installed it became apparent that several changes had been made to the user interface in SpectraSuite ${ }^{\circledR}$. A new data acquisition procedure was developed with the new user interface.

Data acquisition was initiated after the spectral profile for a given flow rate came to equilibrium (Equation 10). The reactor coil is 30 -inches long and the water quench bath is 10 -inches long making the total length of tubing from the start of the reactor coil through the quench bath to the fluorescence flow cell 40 -inches. Given a 1/32-inch inner diameter the total volume of this section of tubing is about $2 \mathrm{ml}$. Thus for every change of flow rate at least $2 \mathrm{ml}$ of solution was required to flow through the reactor for equilibrium to be established. Because this experiment relied on fluorescence in the visible spectrum, the analysis technique was set to the "Scope" setting. In order to calibrate the spectrometer, a dark spectrum and light spectrum were recorded. The light spectrum is a spectral reading recorded from a "blank" sample; in this case unreacted precursor 
solution was used. The light spectrum helps to determine spectrum of the UV light source and inherent fluorescence of the precursor solution. Parameters affecting data acquisition such as integration time, scans to average, and boxcar width are listed on Figure 20. "Integration time" accounts for how long the spectrometer will capture the incoming photons. This variable is synonymous to a camera shutter where shutter speed dictates the amount of exposure. "Scans to average" allows multiple scans to be averaged over time. "Boxcar width" takes the spectral data and averages nearby data points to reduce the background noise of the spectrometer.

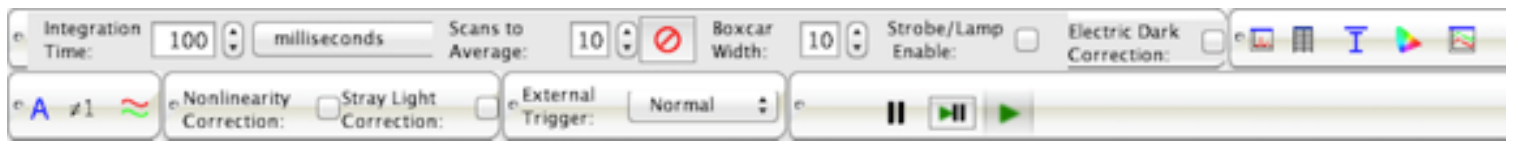

Figure 20: Acquisition controls depicting integration time, boxcar width, and scans to average.

For this experiment, the integration time was set to 100 milliseconds to allow enough photon counts to register a distinct peak wavelength. In initial trials the integration time was set to 500 milliseconds, however, with the addition of a new ultraviolet light source and fixture the peak intensity increased drastically. In segmented continuous flows, there is not much variance between samples once equilibrium is reached but occasionally small bubbles influence the scan so "scans to average" was set to 10 . Due to a presence of noise within the spectrometer, the boxcar width was set to 10 to smooth the spectral profile. SpectraSuite ${ }^{\circledR}$ outputs tab delimited .txt files containing arbitrary count values corresponding to 3648 wavelength values. SpectraSuite ${ }^{\circledR}$ parameters were set to integrate 10 scans recorded every 100 milliseconds apart. Additionally five individual data files were saved 10 seconds apart for every flow rate in every trial. Essentially every file saved is an average of 10 different scans and 5 files are saved (Figure 21). The resulting 
average of these files represents an average of the spectral profile for 50 seconds consisting of 50 individual samples.

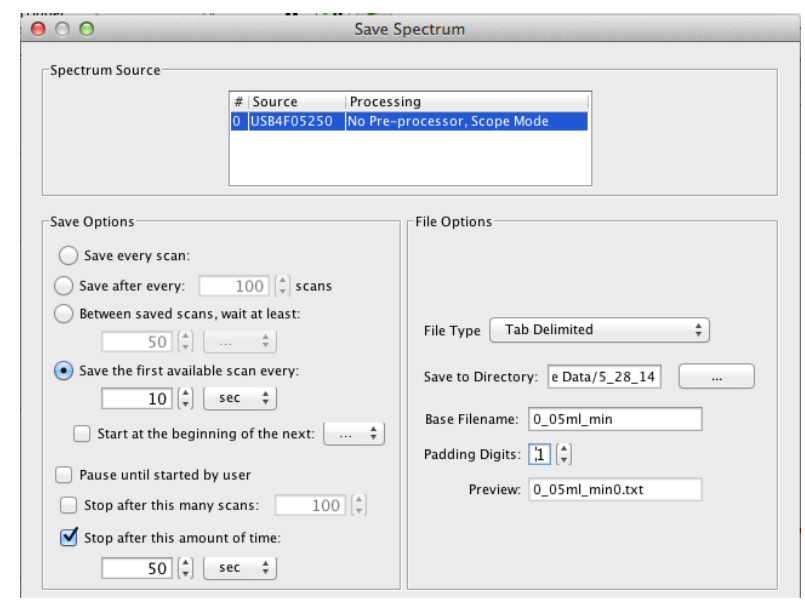

Figure 21: SpectraSuite ${ }^{\circledR}$ save parameters. Note that a scan is saved every 10 seconds for a duration of 50 seconds.

\subsubsection{Data Manipulation with MATLAB}

MATLAB was used to process the raw data output from SpectraSuite ${ }^{\circledR}$. A file tree was created to keep track of the output files (Figure 22). Folders were organized by trial then by flow rate. One folder (Data Not in Sub Folders) contained all SpectraSuite ${ }^{\circledR}$ output files for every flow rate for a trial. In a separate Folder (In Folders), data were organized in subfolders by flowrate. 


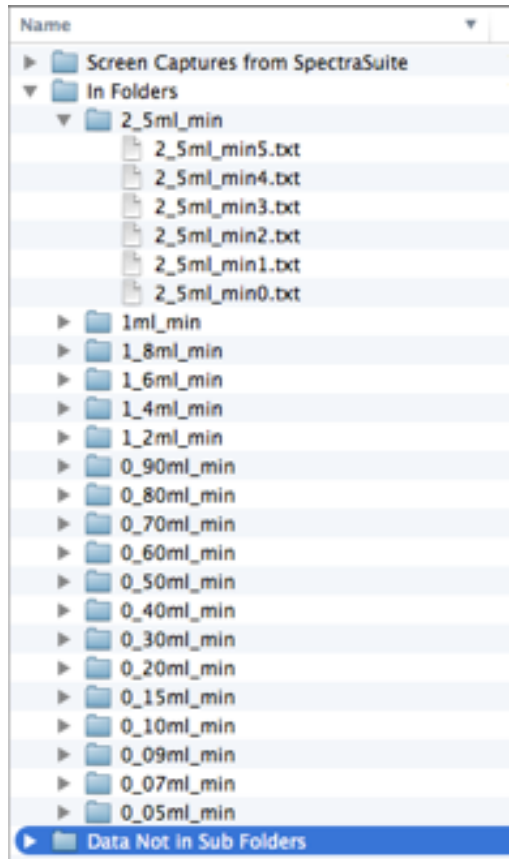

Figure 22: File tree organizing data for MATLAB processing. Note the folder (In Folders) and the folder (Data Not In Sub Folders)

A program was written to search through a folder for text files, import the text file raw data and file name into MATLAB, and organize the data by variable (counts, flowrate (based off the file name), and wavelength). From there the data were plotted on an XY plot with wavelength as the $\mathrm{X}$-axis and counts as the $\mathrm{Y}$-axis (Figure 23). 


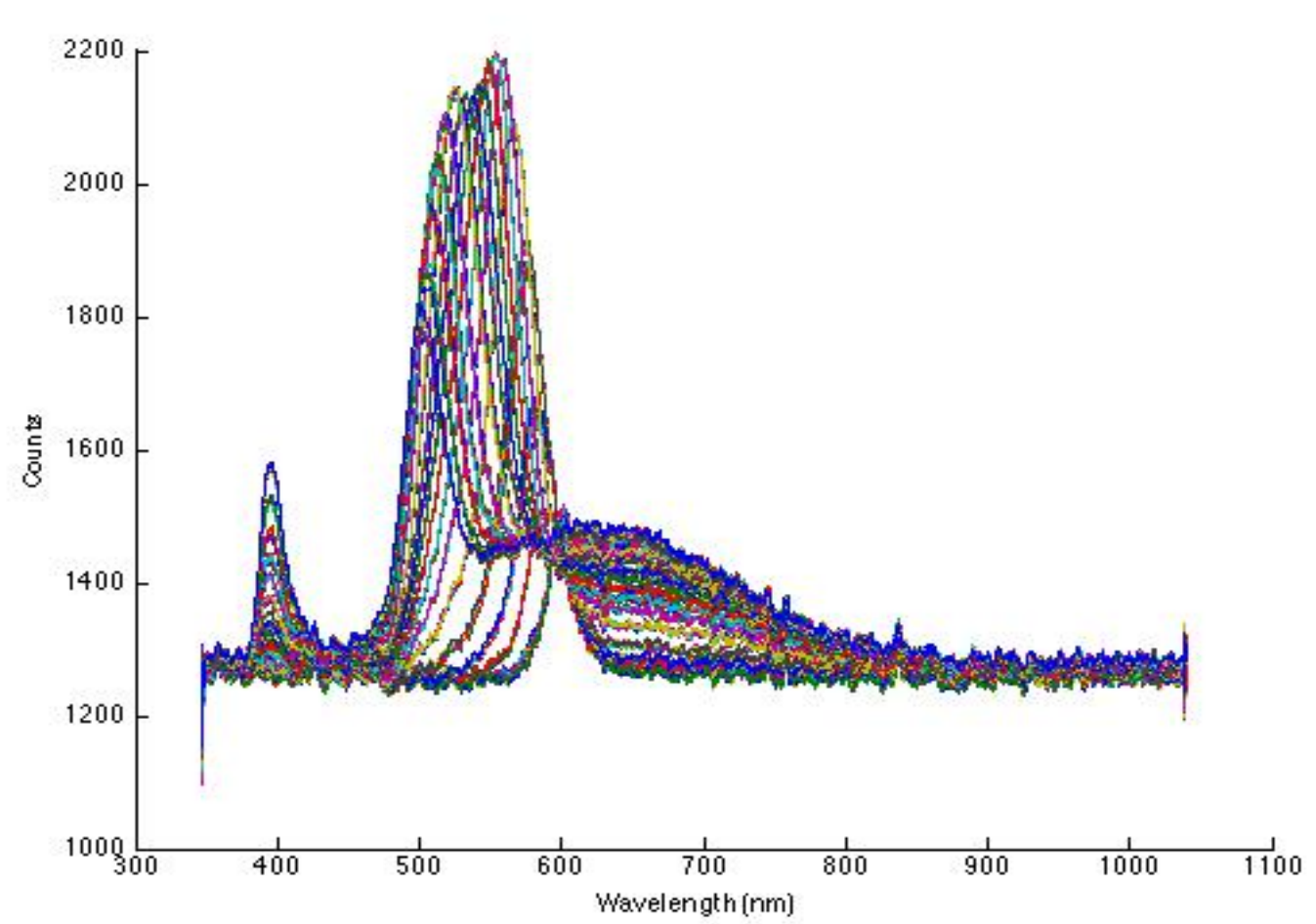

Figure 23: Two-dimensional spectral profile plot. Note each line represents a different SpectraSuite ${ }^{\circledR}$ output file recorded for one synthesis trial.

Two-dimensional spectral profile plots were created for every trial (Appendix A, Appendix F). A three-dimensional topographical surface plot was created from the unappended data of each trial to better visualize the fluorescent peak shift resulting from flowrate modification (Appendix E, Appendix G). As flow rate was increased from $0.05 \mathrm{ml} / \mathrm{min}$ to $2 \mathrm{ml} / \mathrm{min}$ fluorescent peaks shifted toward lower wavelengths (Figure 24). 


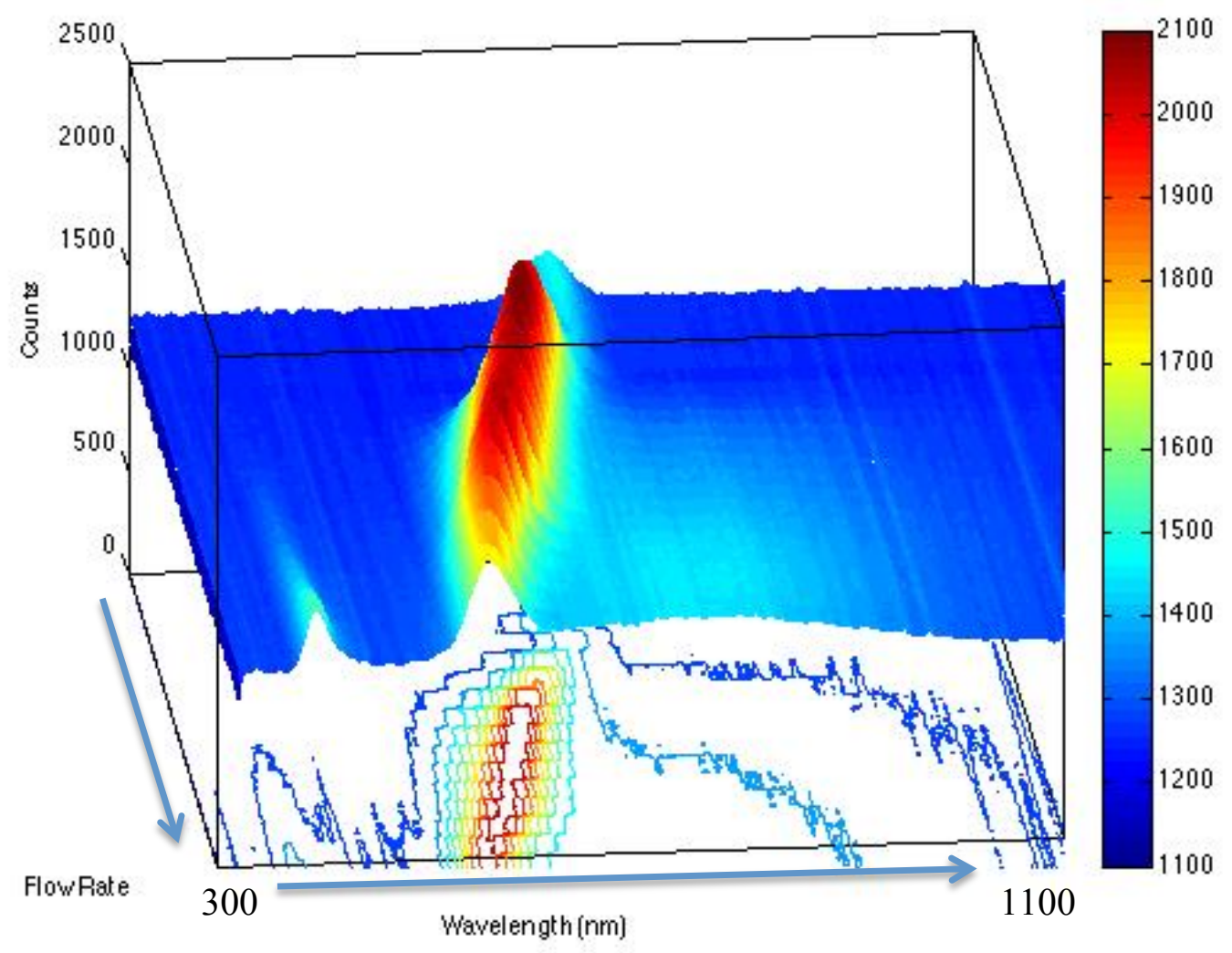

Figure 24: Three-dimensional surface plot generated from aligning the spectral profiles from an entire synthesis trial. Note the shift of the topographic peak as flowrate increases.

The (In Folders) data were then investigated to determine trends within trials. The raw data in each flow rate folder were appended to range from wavelengths of $450 \mathrm{~nm}$ to $650 \mathrm{~nm}$. This range covers all spectral peaks encountered using this synthesis method. A fourth order Gaussian regression was then applied to the data in each flow rate folder. This regression was then plotted against the raw data (Figure 25). 


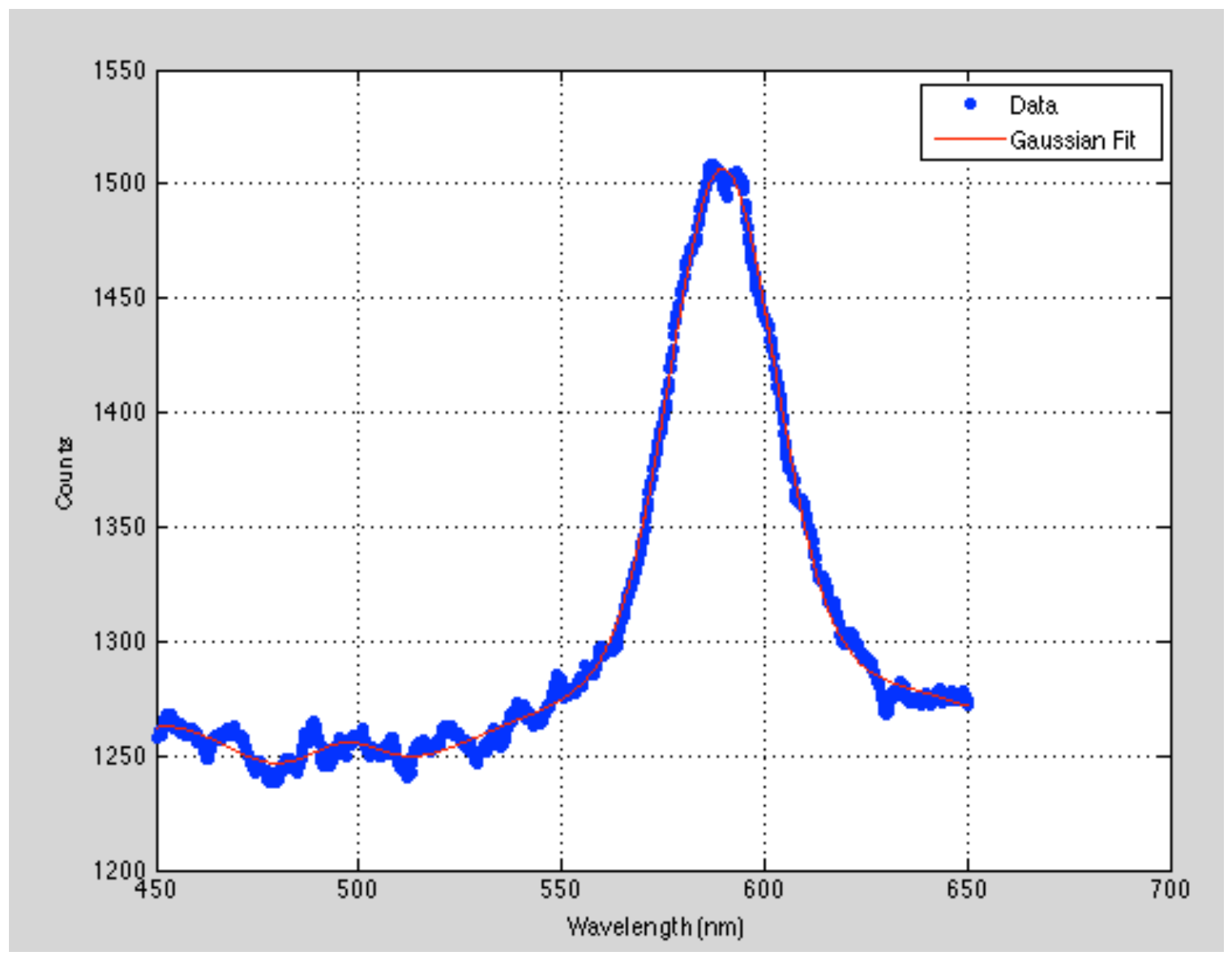

Figure 25: Fourth order Gaussian regression (Red) on raw spectral data (Blue) for a flow rate of $0.15 \mathrm{ml} / \mathrm{min}$ on trial 5_5_14.

The program would then search both the raw data and the regression data for the maximum peak intensity in counts and pull the corresponding wavelength to obtain both an averaged center wavelength and max center wavelength. The raw data, regression data, averaged center wavelength, and max center wavelength were exported to excel for further analysis. 


\subsubsection{Gaussian Regression}

Quantum dots produced with this synthesis method are intended for use in biological imaging and will be viewed by the human eye through various forms of optical microscopy. The human eye can register changes in wavelength under $2 \mathrm{~nm}^{48}$ Any subpeaks near the intensity of the main spectral peak contribute to the perceived color of the synthesized quantum dots. A fourth order Gaussian regression was used in MATLAB to determine the center wavelength of the spectral peak with the greatest intensity at each flowrate. The Gaussian regression functions as a sort of averaging to account for any sub peaks within the main peak area (Figure 26)

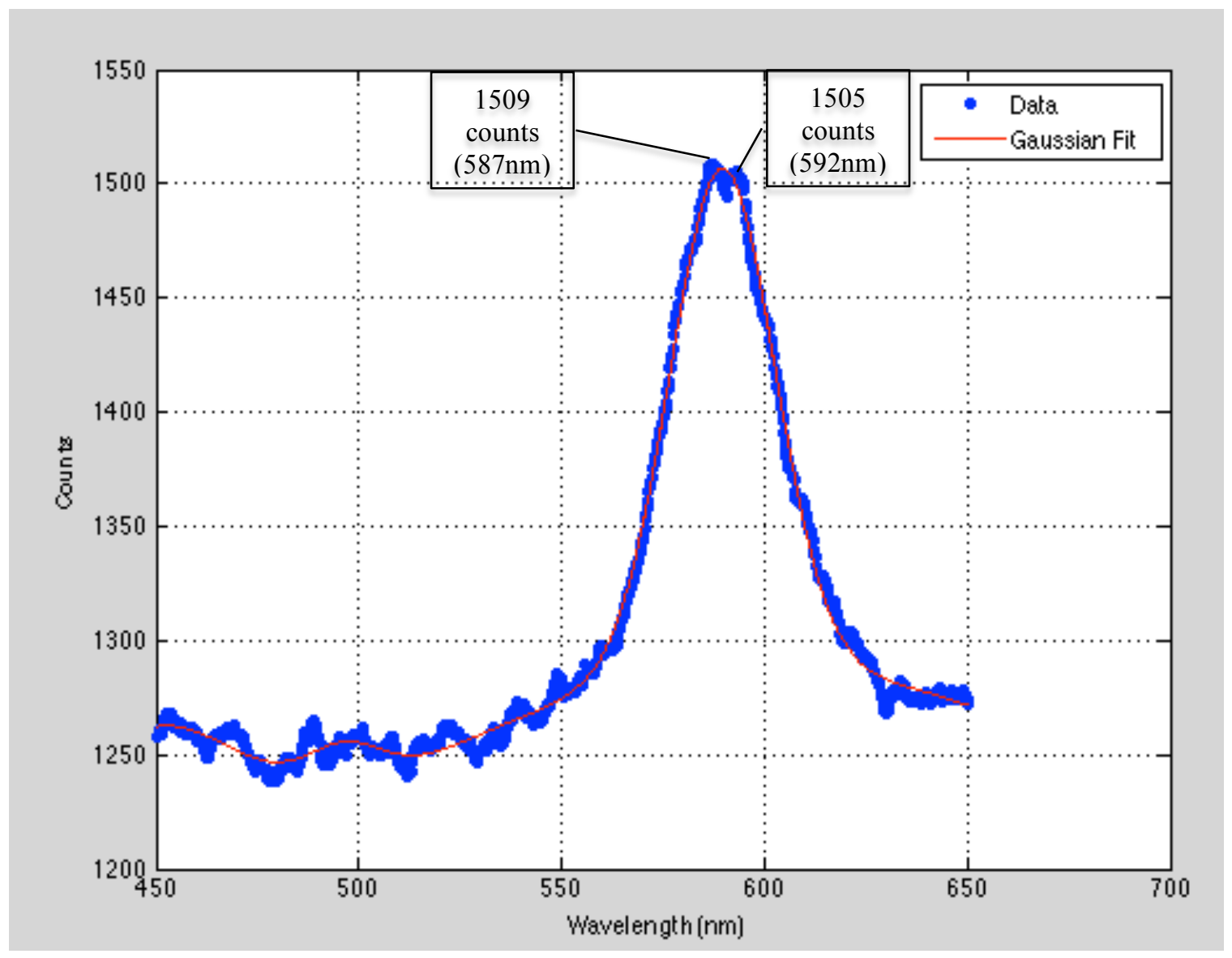

Figure 26: Fourth order Gaussian regression (Red) on raw spectral data (Blue). Note the two sub peaks that make up the main spectral peak. 
For example, the highest peak in Figure 26 above occurs at a wavelength of $587 \mathrm{~nm}$ with an intensity of 1509 arbitrary counts and the second highest peak occurs at a wavelength of $592 \mathrm{~nm}$ with an intensity of 1505 arbitrary counts. These two sub peaks make up the main spectral peak yet they are $5 \mathrm{~nm}$ apart from each other. The Gaussian regression average of these two peaks resulted in a perceived center wavelength of $589 \mathrm{~nm}$. A fourth order regression was used, because it was the lowest order regression that excluded the influence of extraneous peaks of lower intensity elsewhere in the spectrum. The center wavelength determined using this method takes sub peaks and shifts into account giving a more accurate center wavelength.

\subsection{Stainless Steel Reactor Fabrication}

Due to the relatively low thermal conductivity of TEFLON ${ }^{\circledR}$ tubing at $0.242 \mathrm{~W} / \mathrm{m}^{\circ} \mathrm{C}, 316$ stainless steel was used to fabricate a reaction coil. ${ }^{37}$ It was theorized that the relatively low thermal conductivity of TEFLON ${ }^{\circledR}$ would detrimentally affect thermal transfer to the reactant solution. This would result in a large thermal gradient leading to less monodispersity of the nanocrystalline nuclei. 316 stainless steel, in comparison, has a thermal conductivity of $13 \mathrm{~W} / \mathrm{m}^{\circ} \mathrm{C}, 50$ times that of TEFLON ${ }^{\circledR} \cdot{ }^{37}$ A 30 -inch length of 316 stainless steel was obtained to fabricate a reactor coil. The stainless steel would not plastically deform to maintain a coil shape so a heat treatment was performed to recrystallize the steel in a coil shape. 


\subsubsection{Heat Treatment}

316 stainless tubing was bent to maintain a coil shape through a recrystallization heat treatment. The sample was wrapped around a steel jig and secured in place using clamps made out of bolts and washers (Figure 27).

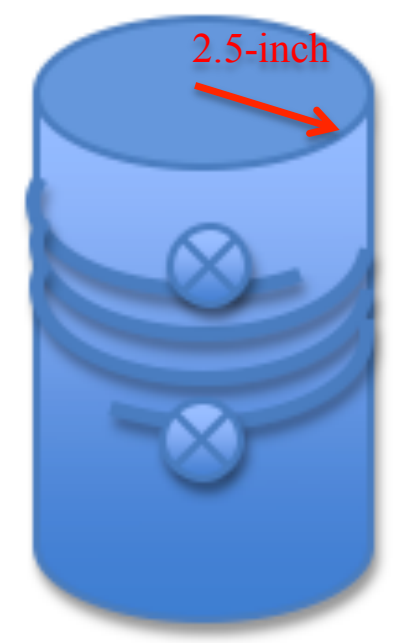

Figure 27: Steel recrystallization jig with a 2.5-inch outer diameter. Note Phillips screws used to secure tubing.

The jig was then heated for four hours at $1100^{\circ} \mathrm{C}$ and quenched in a water bath. The tubing retained the desired shape and was implemented in the reactor. This reactor coil was implemented based on the assumption of improved performance; however, after thorough testing this assumption proved false. It became apparent that the thermal treatment had caused the tubing to grow an oxide. The spalling and cracking of this oxide is thought to influence the nucleation and growth characteristics of the reactor. An analysis of surface roughness was later done to further investigate this hypothesis. 


\section{OPTIMIZED QD REACTOR RESULTS, STAINLESS STEEL REACTOR}

A total of eight trials were run with the optimized 316 stainless steel reactor using the optimized experimental procedure. Two and three-dimensional plots were created to compare reaction characteristics and general trends between trials (Appendix AAppendix G). Microsoft Excel was used to create additional plots from MATLAB data output to highlight general trends. As shown on Figure 28 eight flow rates were selected to show the shifts in center wavelength and relative peak intensities that occur as a result of varying flow rate.

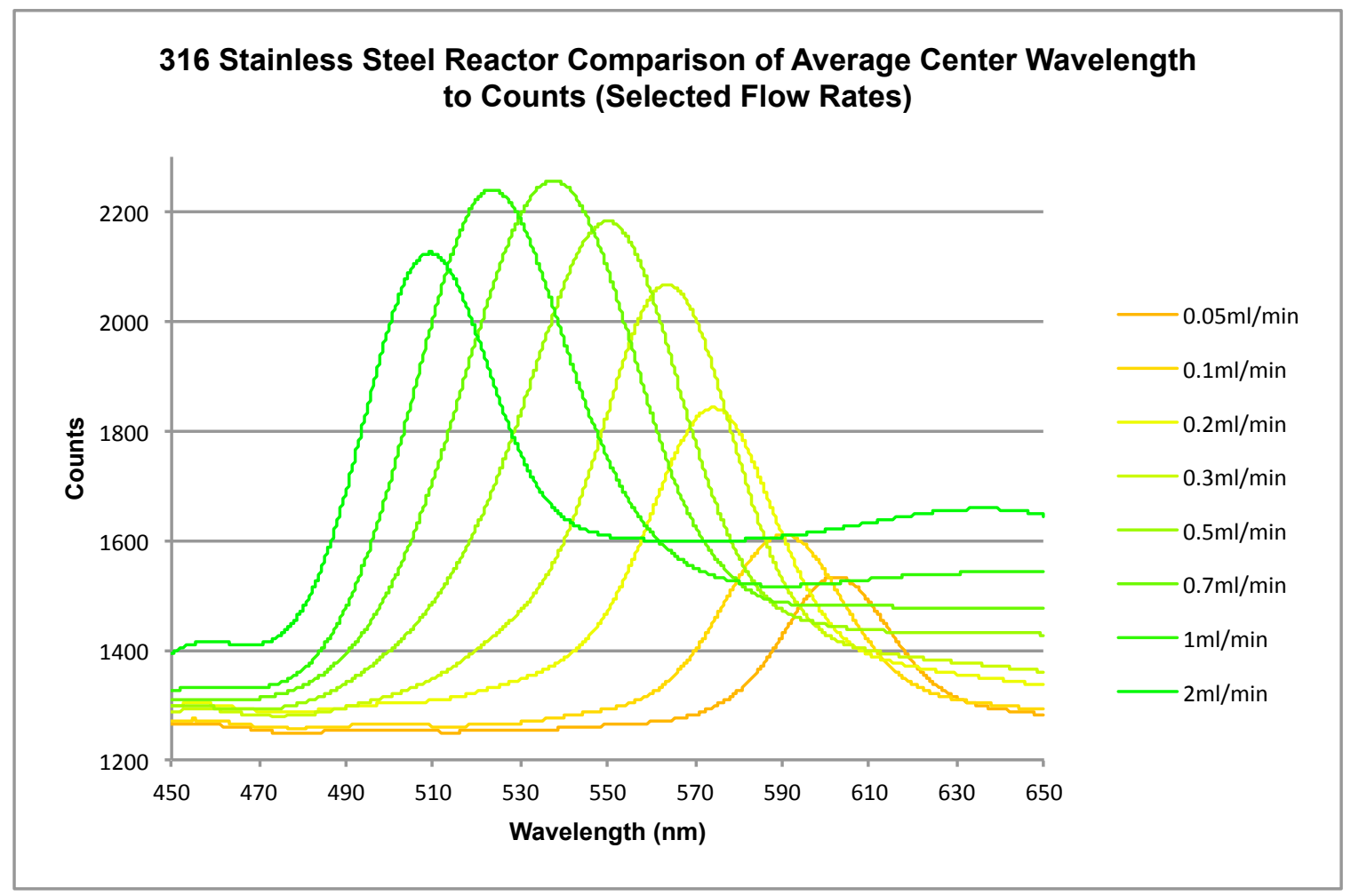

Figure 28: View of average spectral profiles at selected flow rates for all eight trials. Note that the center wavelength for each peak were converted to RGB color values and included as the flow rate color. Each spectral peak's color is the RGB equivalent of the color the synthesized quantum dots fluoresce. 
The color of each flowrate on the figure above corresponds to the center wavelength of the spectral peak for that flowrate. The center wavelengths for each flowrate are listed below (Table VI).

Table VI: Average center wavelength for selected flow rates

\begin{tabular}{|c|c|}
\hline Flow Rate (ml/min) & Center Wavelength (nm) \\
\hline 0.05 & 600 \\
\hline 0.1 & 587 \\
\hline 0.2 & 571 \\
\hline 0.3 & 561 \\
\hline 0.5 & 545 \\
\hline 0.7 & 533 \\
\hline 1 & 520 \\
\hline 2 & 507 \\
\hline
\end{tabular}

As flow rate increases the base intensity for the spectral peaks increases from about 1250 counts to 1400 counts on the left of the spectral peaks and from 1250 counts to over 1600 counts on the right of the spectral peaks. At increased flow rates the overall spectral intensity in the wavelength range from $450 \mathrm{~nm}$ to $650 \mathrm{~nm}$, increases. The increase is disproportionately skewed, with a greater increase in intensity to the right of the spectral peaks. This phenomenon suggests that polydispersity increases with increasing flow rate and will be investigated further in the discussion section below. To better see the left shift of the fluorescent profile with increasing flow rate, the previous figure was normalized (Figure 29). 


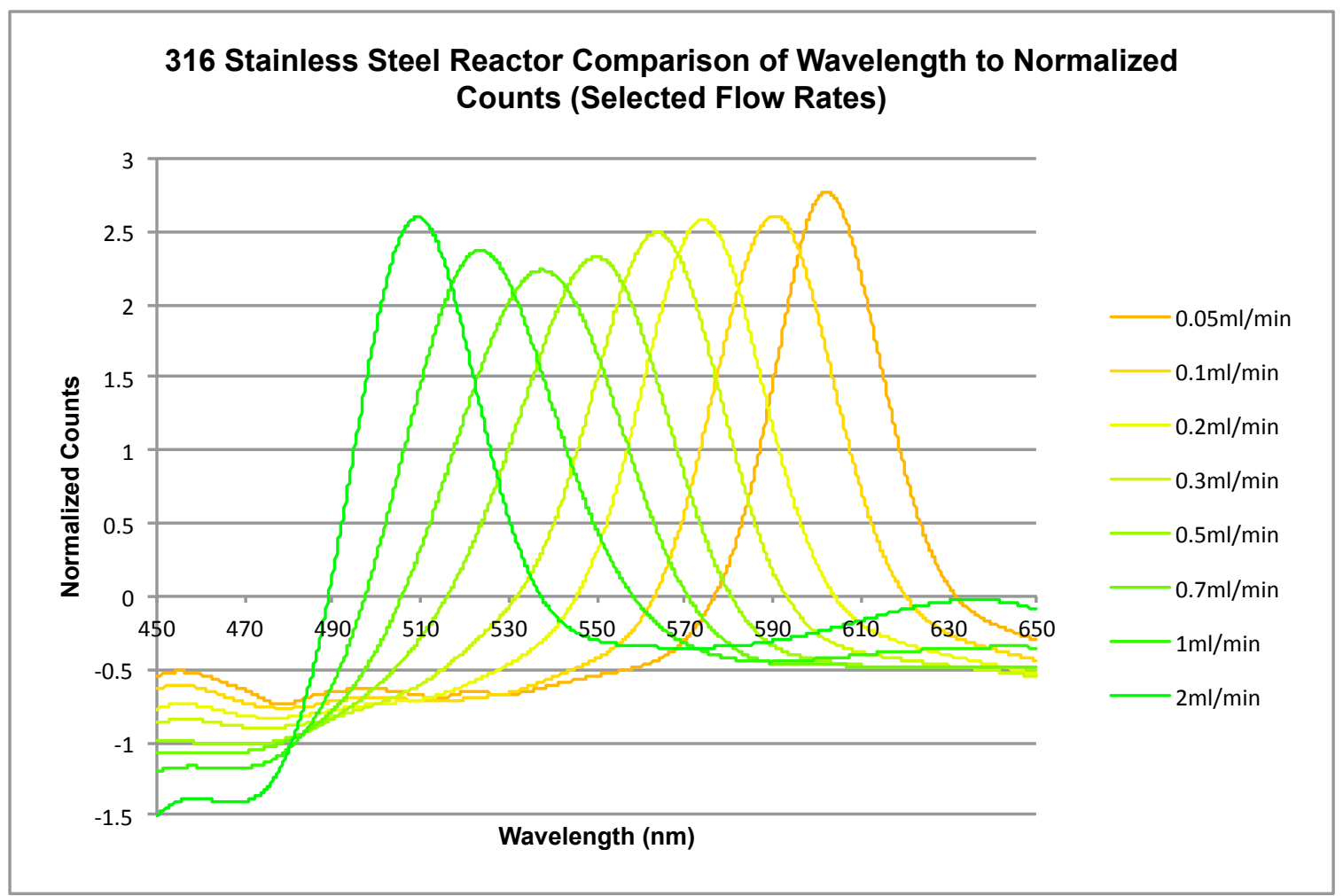

Figure 29: Normalized view of average spectral profiles of selected flow rates for all eight trials.

\subsection{Relationship of Flow Rate to Peak Center Wavelength}

The center wavelengths for all eight 316 stainless steel reactor trials were plotted against flowrate to show differences between the individual trials (Figure 30). 


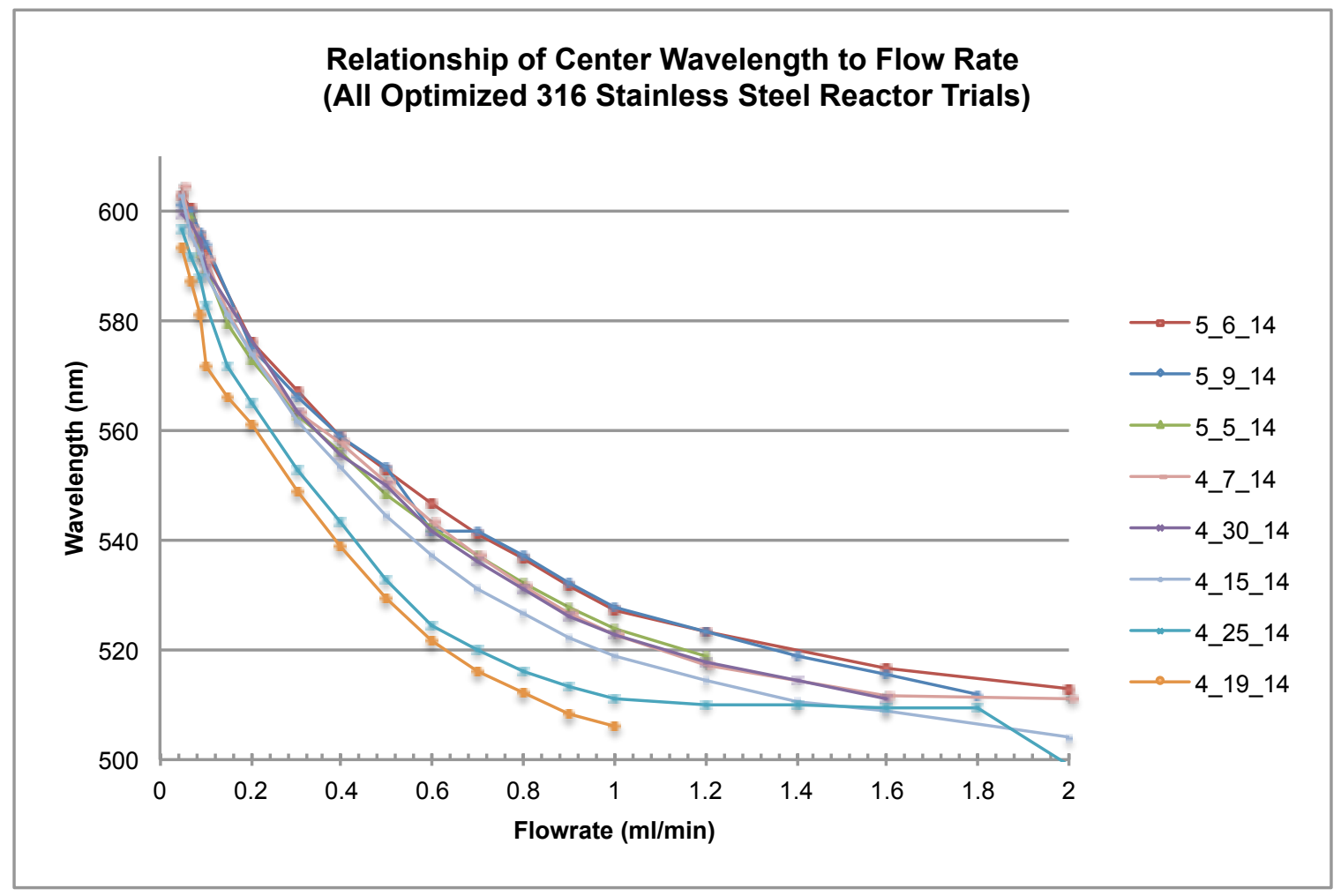

Figure 30: Center spectral peak wavelength plotted by trial for all reagent flowrates. Note: Trial 4_25_14 was run with half of the standard volume of the TOP-Se precursor solution. Trial 4_19_14 was run at a reaction temperature of $230^{\circ} \mathrm{C}$ causing a shift in center wavelength of the spectral peaks at each flowrate.

Trial 4_19_14 (Orange) occurred at a reaction temperature of $230^{\circ} \mathrm{C}$ and the center wavelength distribution shifted toward smaller wavelengths. Suggesting that lowering the reaction temperature reduces the rate at which the reaction can occur. The implications of this are that the reaction will proceed more slowly, creating smaller nanocrystals given the same residence time. Trial 4_19_14 and trial 4_15_14 were conducted with a reduced volume of the TOP-Se precursor solution. In trial 4_19_14 half the TOP-Se precursor solution was spilled. In trial 4_15_14 a defective rubber septum leaked a small amount of TOP-Se precursor solution. Both trials indicate a shift toward smaller wavelengths and thus smaller quantum dots. The remaining trials display a tight distribution indicating process repeatability. 
Microsoft Excel was used to generate an empirical equation relating the average center wavelength of all stainless steel reactor trials to flow rate (Figure 31).

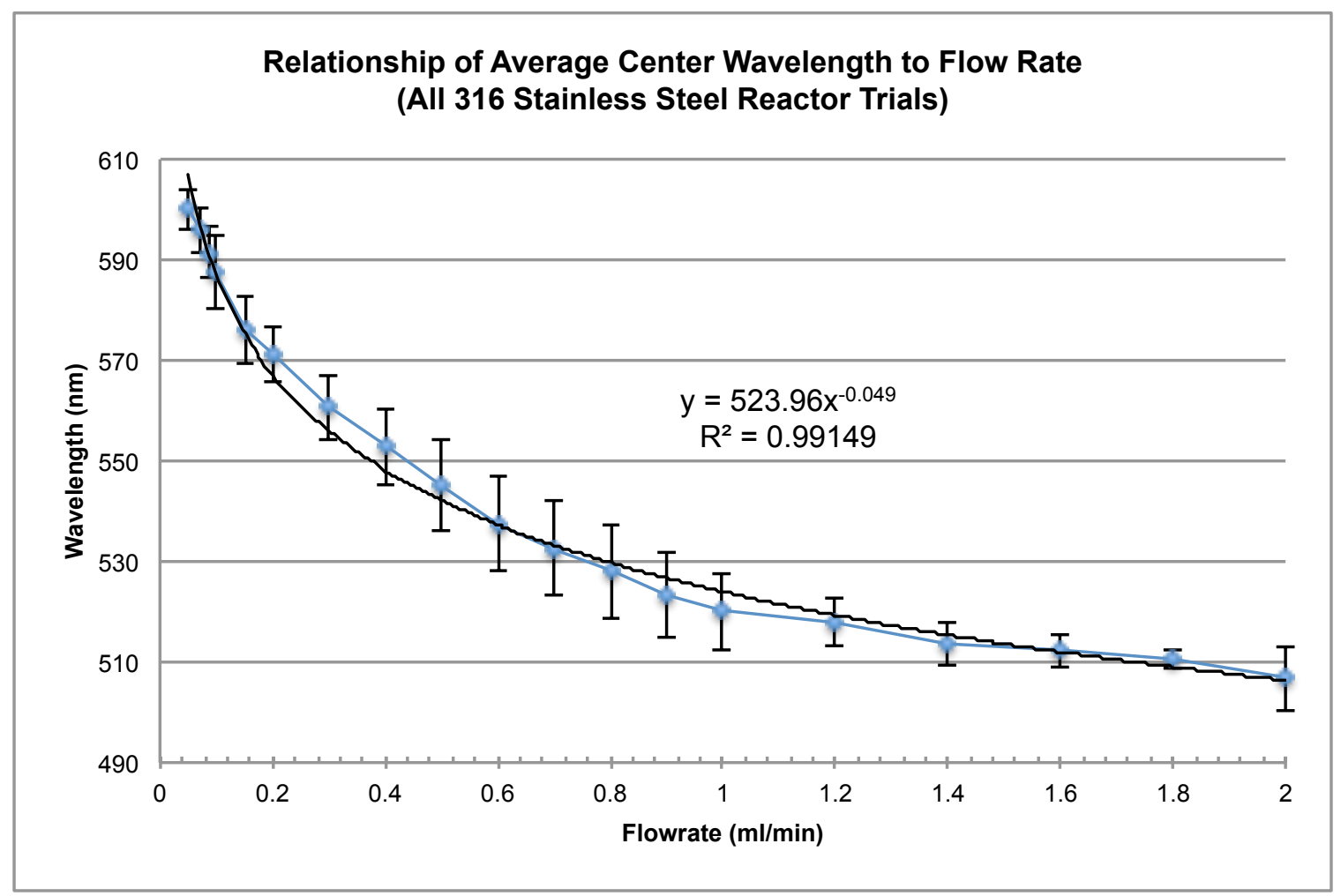

Figure 31: Relationship of flow rate to the average center wavelength for all trials. Note the error bars show one positive and negative standard deviation of center wavelength.

The equation will allow for precise control of wavelength and size of quantum dots utilizing flow rate as the primary variable (Equation 11).

Equation 11: Power function relationship between average center wavelength and flowrate

$$
\lambda_{\begin{array}{c}
\text { Average Center } \\
\text { Wavelength }(\mathrm{nm})
\end{array}}=523.96\left(Q_{\text {Volumetric }_{\text {Flow Rate }(\mathrm{ml} / \mathrm{min})}}\right)^{-0.049}
$$

The center wavelength of the peaks followed a power function regression with an $\mathrm{R}^{2}$ value of 0.99 . Equation 11 is both a better fit and takes into account a larger data set than 
the previously introduced Equation 8 and is thought to be a more accurate representation of the relationship between average center wavelength and flowrate.

\subsection{Analysis of Particle Diameter}

Quantum dots ranging in size from $4.6 \mathrm{~nm}$ to $2.4 \mathrm{~nm}$ were synthesized in the optimized 316 stainless steel reactor (Figure 32). The size distribution was determined using an empirical equation developed at the University of Arkansas (Equation 9). ${ }^{46}$

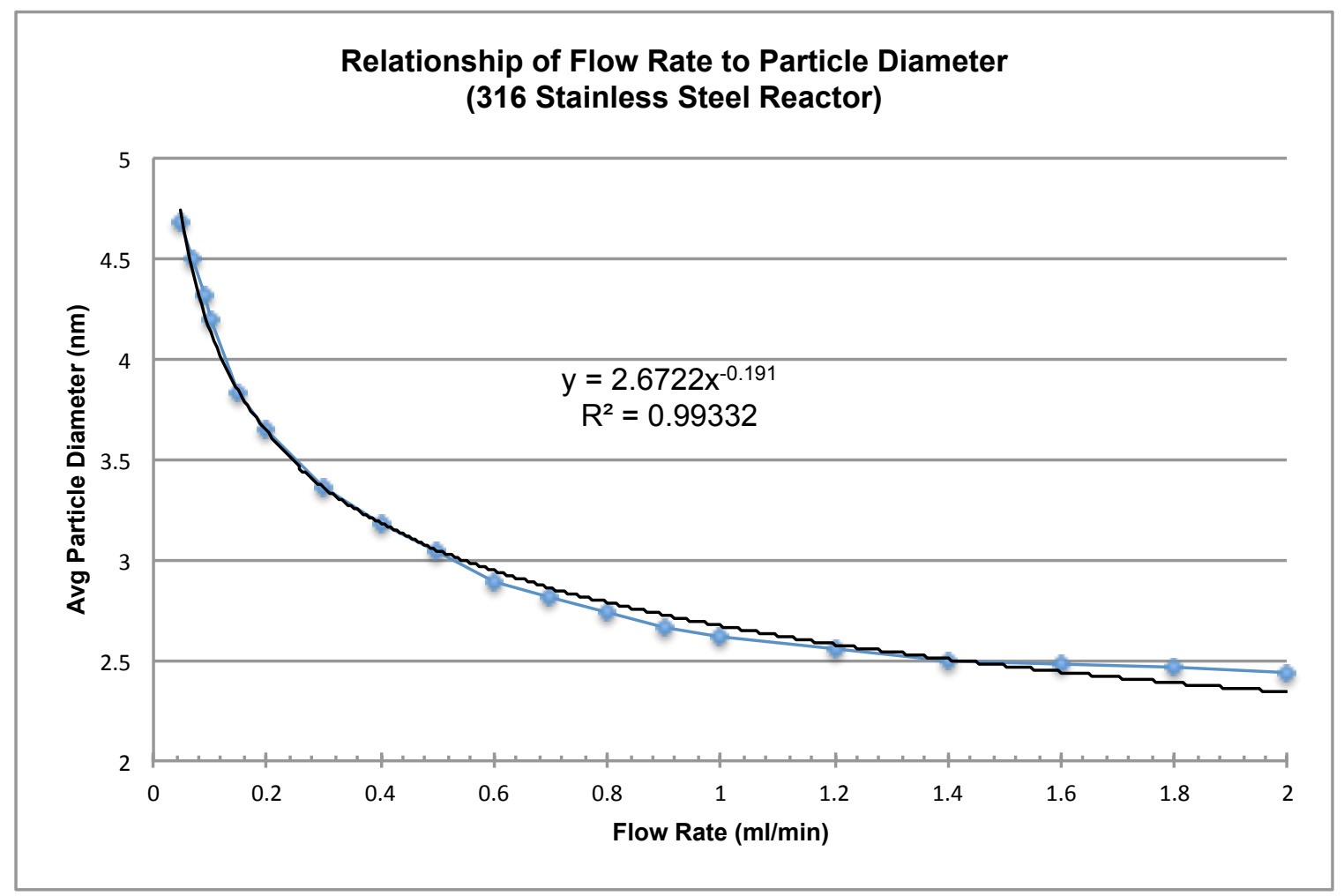

Figure 32: Average particle diameter for each tested flow rate (316 stainless steel reactor).

Average particle diameter increases with decreasing flow rate. 


\subsection{Repeatability}

The standard deviation between all experimental trials for the stainless steel reactor was calculated for the assessed flow rates (Table IV).

Table VII: Calculated standard deviation of center wavelength at tested flow rates.

\begin{tabular}{|c|c|}
\hline $\begin{array}{c}\text { Flow Rate } \\
(\mathbf{m l} / \mathbf{m i n})\end{array}$ & $\begin{array}{c}\text { Standard Deviation of Center } \\
\text { Wavelength } \mathbf{( n m})\end{array}$ \\
\hline 0.05 & 4 \\
\hline 0.07 & 5 \\
\hline 0.09 & 5 \\
\hline 0.1 & 7 \\
\hline 0.15 & 7 \\
\hline 0.2 & 6 \\
\hline 0.3 & 6 \\
\hline 0.4 & 8 \\
\hline 0.5 & 9 \\
\hline 0.6 & 9 \\
\hline 0.7 & 10 \\
\hline 0.8 & 9 \\
\hline 0.9 & 9 \\
\hline 1 & 8 \\
\hline 1.2 & 5 \\
\hline 1.4 & 4 \\
\hline 1.6 & 3 \\
\hline 1.8 & 2 \\
\hline 2 & 6 \\
\hline Average StDev & 6 \\
\hline
\end{tabular}

The average standard deviation was $6 \mathrm{~nm}$ and the maximum standard deviation is $10 \mathrm{~nm}$.

The larger sample size for the stainless steel reactor compared to the Proof of Concept Reactor provided a larger opportunity for human error. Three of the trials used in the calculations of this section were substandard. Trial 4_19_14 occurred at a reaction temperature of $230^{\circ} \mathrm{C}$ instead of the standard $240^{\circ} \mathrm{C}$. Trial 4_19_14 and Trial 4_15_14 were conducted with a reduced volume of the TOP-Se precursor solution. Half of the TOP-Se precursor solution was spilled in trial 4_19_14. A defective rubber septum 
leaked a small amount of TOP-Se precursor solution in trial 4_15_14. A revised standard deviation table was created excluding these botched trials (Table VIII).

Table VIII: Calculated standard deviation of center wavelength at tested flow rates excluding erroneous trials.

\begin{tabular}{|c|c|}
\hline $\begin{array}{c}\text { Flow Rate } \\
(\mathbf{m l} / \mathbf{m i n})\end{array}$ & $\begin{array}{c}\text { Standard Deviation of Center } \\
\text { Wavelength (nm) }\end{array}$ \\
\hline 0.05 & 2 \\
\hline 0.07 & 2 \\
\hline 0.09 & 2 \\
\hline 0.1 & 2 \\
\hline 0.15 & 1 \\
\hline 0.2 & 1 \\
\hline 0.3 & 2 \\
\hline 0.4 & 2 \\
\hline 0.5 & 3 \\
\hline 0.6 & 3 \\
\hline 0.7 & 4 \\
\hline 0.8 & 4 \\
\hline 0.9 & 4 \\
\hline 1 & 3 \\
\hline 1.2 & 3 \\
\hline 1.4 & 4 \\
\hline 1.6 & 3 \\
\hline 1.8 & 1 \\
\hline 2 & 5 \\
\hline Average StDev & 3 \\
\hline
\end{tabular}

The new average standard deviation is $3 \mathrm{~nm}$ and the maximum standard deviation is $5 \mathrm{~nm}$. These values better represent the process reliability and show an increase of reliability in comparison to the Proof of Concept Reactor. The modified synthesis process exhibits a high degree of accuracy for producing desired central wavelengths. 


\section{OPTIMIZED QD REACTOR RESULTS, TEFLON ${ }^{\circledR}$ REACTOR $^{-}$}

A total of four trials were run with the optimized TEFLON ${ }^{\circledR}$ reactor using the optimized experimental procedure. Two and three-dimensional plots were created to compare reaction characteristics and general trends between trials (Appendix F-Appendix G). Microsoft Excel was used to create additional plots from MATLAB data output to highlight general trends. Thirteen flow rates were selected to show the shifts in center wavelength and relative peak intensities that occur as a result of varying flow rate (Figure 33).

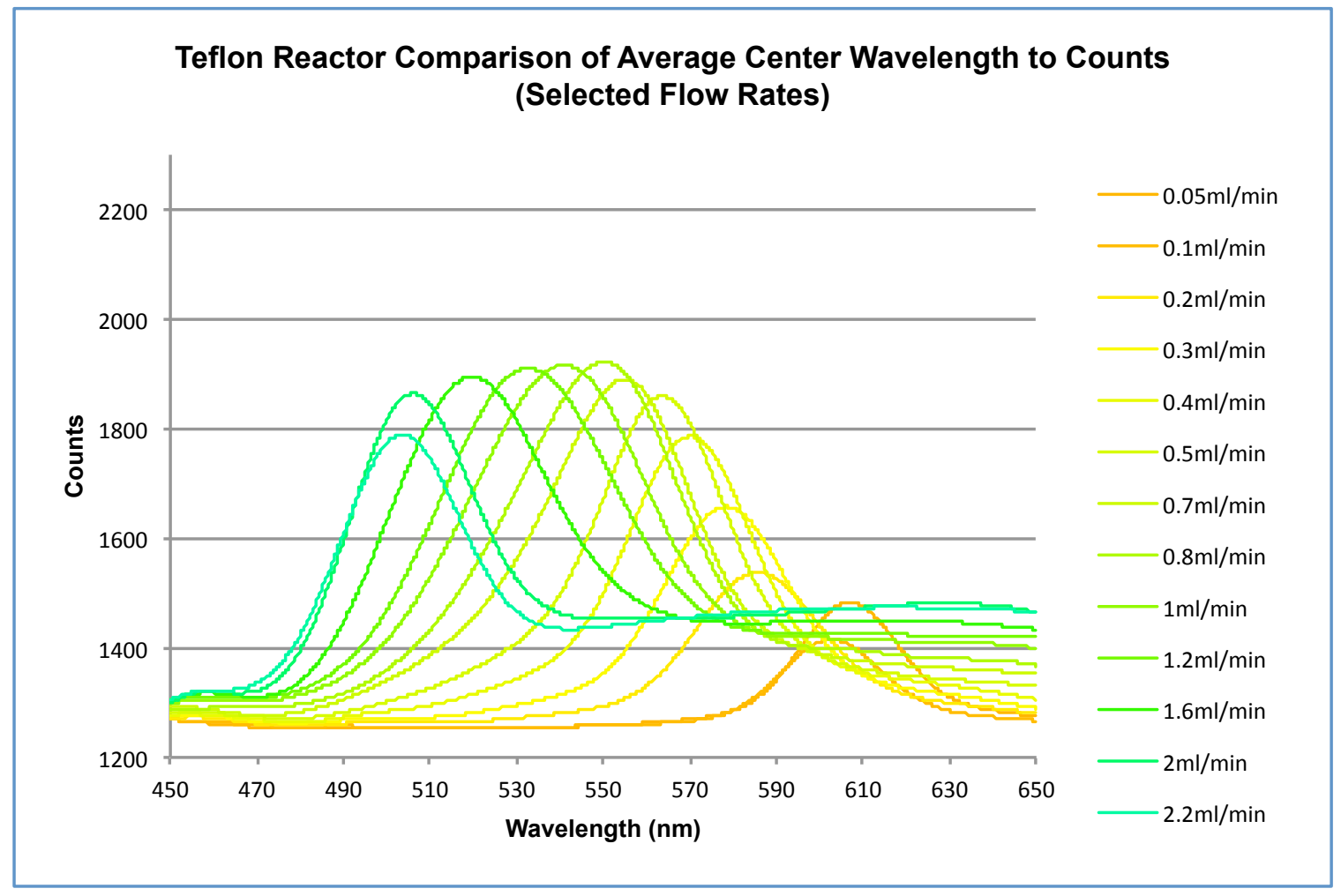

Figure 33: View of average spectral profiles at selected flow rates for all four trials. Note that the center wavelength for each peak was converted to RGB color values and included in the plot. Each spectral peak's color is the RGB equivalent of the color the synthesized quantum dots fluoresce.

The color of each flowrate in the figure above corresponds with the center wavelength of 
the spectral peak of said flowrate. The center wavelengths for each flowrate are listed below (Table IX).

Table IX: Average center wavelength for selected flow rates

\begin{tabular}{|c|c|}
\hline Flow Rate (ml/min) & Center Wavelength (nm) \\
\hline 0.05 & 607 \\
\hline 0.1 & 604 \\
\hline 0.2 & 587 \\
\hline 0.3 & 580 \\
\hline 0.4 & 571 \\
\hline 0.5 & 565 \\
\hline 0.7 & 555 \\
\hline 0.8 & 550 \\
\hline 1 & 541 \\
\hline 1.2 & 534 \\
\hline 1.6 & 521 \\
\hline 2 & 506 \\
\hline 2.2 & 504 \\
\hline
\end{tabular}

As flow rate increases, the base intensity for the spectral peaks increases from about 1275 counts to 1300 counts on the left of the spectral peaks and from 1275 counts to about 1500 counts on the right of the spectral peaks (Figure 33). As with the P.O.C Reactor and optimized stainless steel reactor, increasing the flow rate increases the overall spectral intensity in the wavelength range from $450 \mathrm{~nm}$ to $650 \mathrm{~nm}$. Again, the increase is disproportionately skewed, with a greater increase in intensity to the right of the spectral peaks. The relative intensity of this increase is less than that experienced by the 316 stainless steel reactor, suggesting that polydispersity increases with increasing flow rate to a lesser degree in the TEFLON ${ }^{\circledR}$ reactor. To better see the left shift of the fluorescent profile with increasing flow rate the previous figure was normalized (Figure 34). 


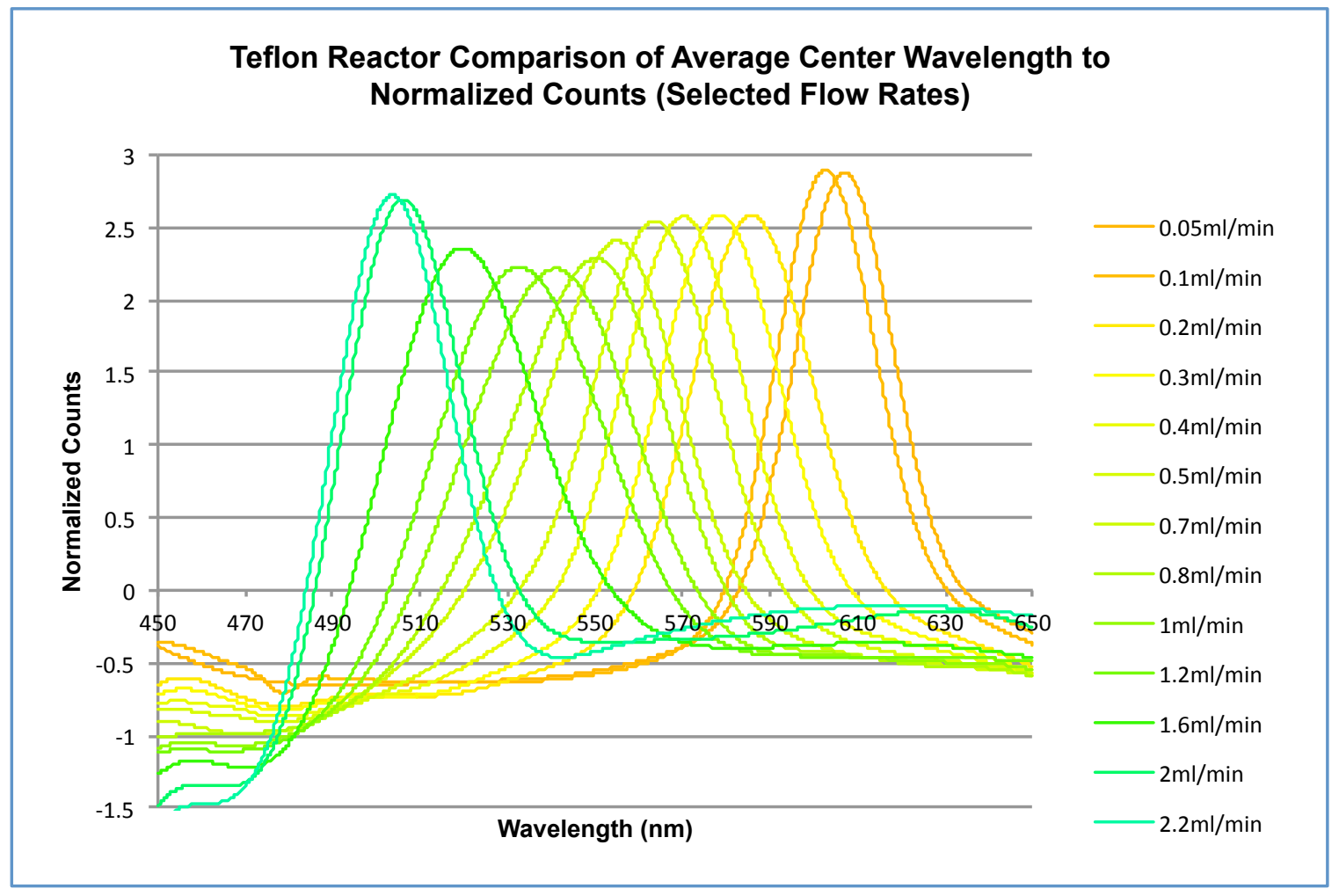

Figure 34: Normalized view of average spectral profiles of selected flow rates for all four trials.

Normalization was accomplished using the normalization function in Microsoft Excel.

Normalizing the data makes it easier to see the wavelength shift corresponding to increasing flowrate.

In trial 6_23_14 the hotplate thermal probe was accidentally knocked out of the high temperature silicon oil reactor bath. This caused temperature of the reaction bath to increase to $265^{\circ} \mathrm{C}$. Data were collected for the $0.05 \mathrm{ml} / \mathrm{min}$ and $0.07 \mathrm{ml} / \mathrm{min}$ flow rates before the bath temperature was noticed. The $0.05 \mathrm{ml} / \mathrm{min}$ flow rate produced a quantum dots solution with a center wavelength of $618 \mathrm{~nm}$ and the $0.07 \mathrm{ml} / \mathrm{min}$ flow rate produced a quantum dot solution with a center wavelength of 614nm (Figure 35). 


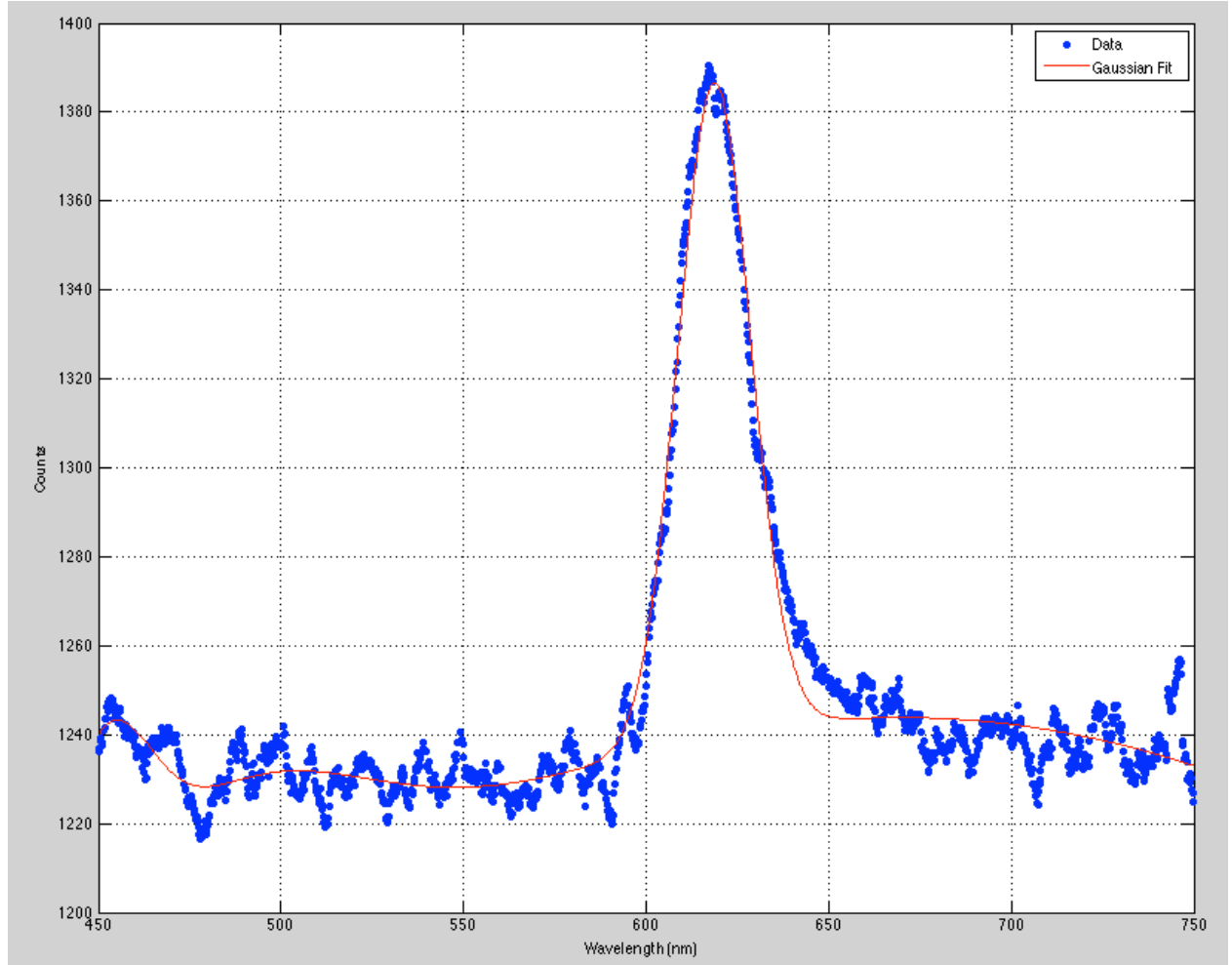

Figure 35: Quantum dot fluorescence peak $(618 \mathrm{~nm})$ at a flow rate of $0.05 \mathrm{ml} / \mathrm{min}$. Note this synthesis occurred at a temperature of $265^{\circ} \mathrm{C}$

Based on the results presented, by increasing reactor temperature, larger quantum dots can be synthesized, however, the maximum working temperature of TEFLON ${ }^{\circledR}\left(271^{\circ} \mathrm{C}\right)$ cannot be exceeded.

\subsection{Relationship of Flow Rate to Peak Center Wavelength}

The center wavelengths for all four $\mathrm{TEFLON}^{\circledR}$ reactor trials were plotted against flowrate to show differences between individual trials (Figure 36). 


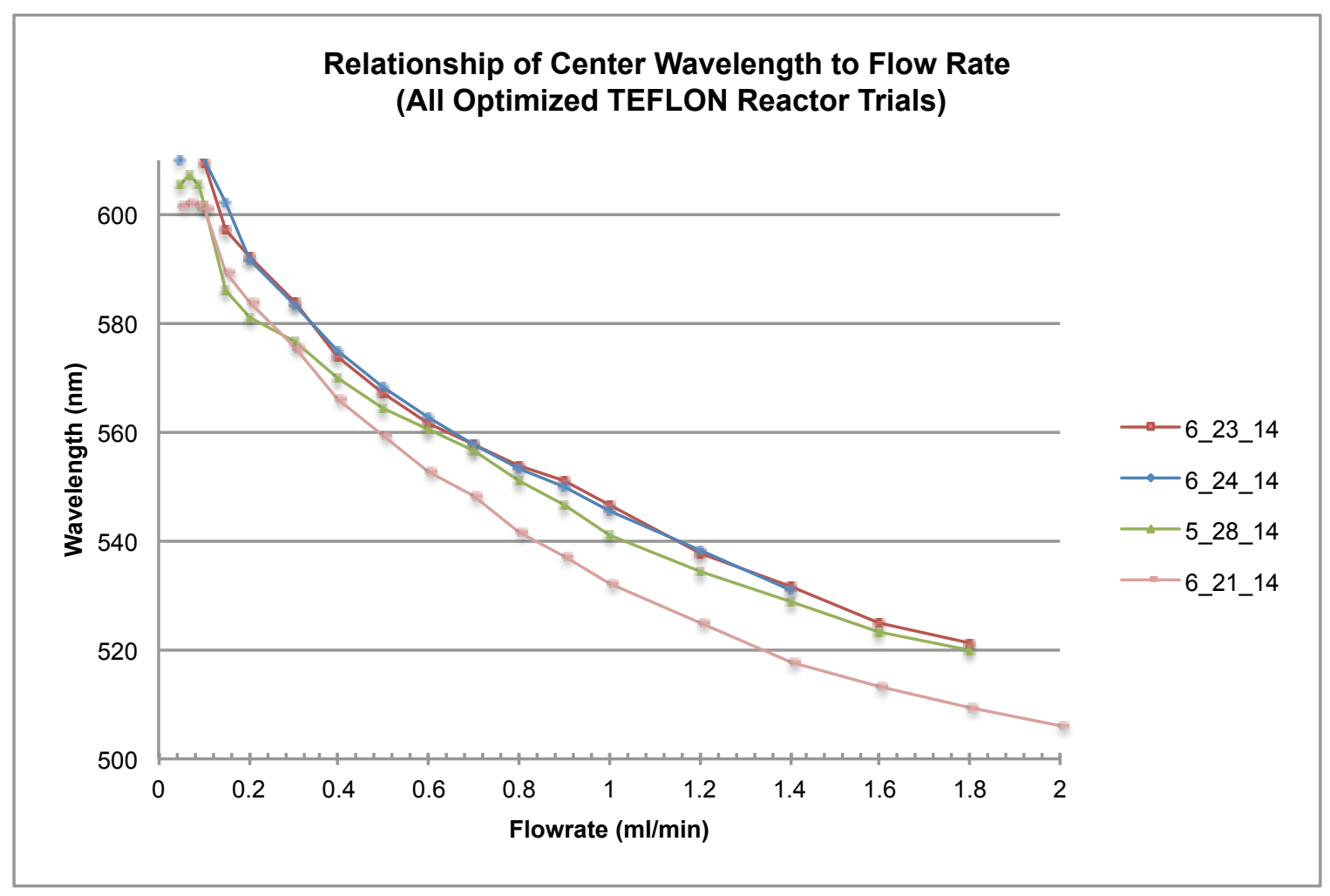

Figure 36: Center spectral peak wavelength plotted by trial for all reagent flowrates. Note: Trial 5_28_14 and trial 6_21_14 were run with old octadecene while trial 6_23_14 and trial 6_24_14 were run with new octadecene.

Trial 5_28_14 and trial 6_21_14 were run with octadecene purchased 5 years ago in 2009. This old octadecene could have degraded or become contaminated which could explain the differences seen in the figure above. Trial 6_23_14 and trial 6_24_14 were run with new octadecene purchased from Sigma Aldrich in 2014. Another cause of the discrepancies between trials could have been the position of the fume hood shield. It was discovered during trial 5_28_14 that thermal characteristics of the reaction bath vary with the position of the fume hood-sliding shield. When the shield is lowered air is pulled at a higher velocity past the heating bath and the bath temperature was found to fluctuate up to $10^{\circ} \mathrm{C}$ based on hood position alone. A mark on the fume hood was used to ensure proper positioning for all trials once this setback was discovered. The flow rates that had 
been affected were retested and the data displayed on Figure 36 is free of this error. The new octadecene trials display a tight distribution indicating process repeatability. Two trials, however, do not constitute a large enough sample size to make this claim with certainty. Data from all four trials were analyzed using Microsoft Excel to generate an empirical equation relating the average center wavelength of all TEFLON ${ }^{\circledR}$ reactor trials to flow rate (Figure 37).

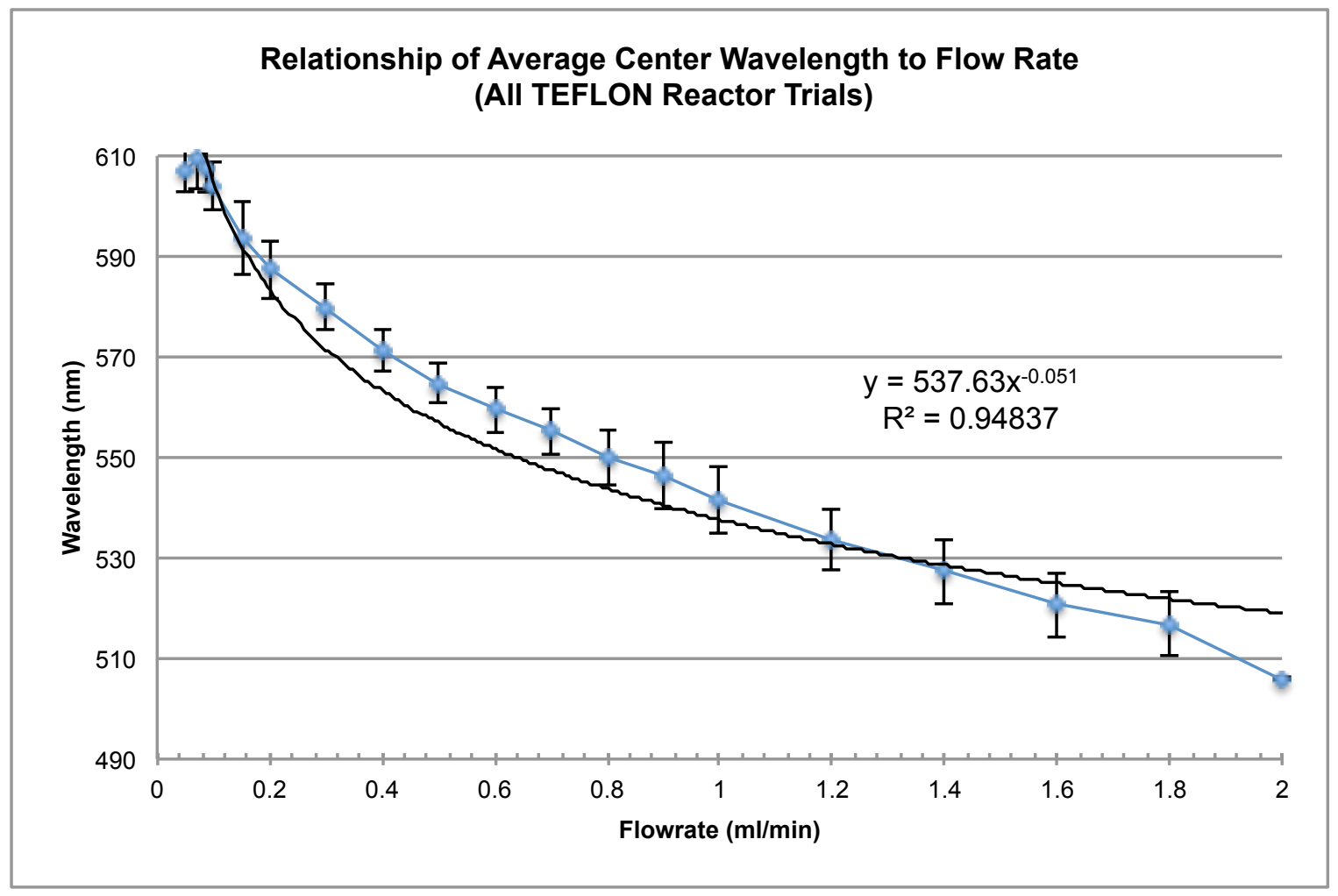

Figure 37: Relationship of flow rate to the average center wavelength for all TEFLON ${ }^{\circledR}$ reactor trials. Note the error bars show one positive and one negative standard deviation of center wavelength.

Equation 12 will allow for precise control of wavelength and size of quantum dots utilizing flow rate as the primary variable for the optimized TEFLON ${ }^{\circledR}$ reactor 
Equation 12: Optimized TEFLON ${ }^{\circledR}$ reactor power function relationship between average center wavelength and flowrate

$$
\lambda_{\text {Average Center }}=537.63\left(Q_{\text {Volumelength }(\mathrm{nm})}^{Q_{\text {Flow Rate }(\mathrm{ml} / \mathrm{min})}}\right)^{-0.051}
$$

The center wavelength of the peaks followed a power function regression with an $\mathrm{R}^{2}$ value of 0.95 . The trend line represented by Equation 12 does not represent as good a fit as Equation 8. This is likely because of the use of new octadecene as a base solvent during the last two optimized TEFLON ${ }^{\circledR}$ reactor trials.

\subsection{Analysis of Particle Diameter}

Quantum dots ranging in size from $4.9 \mathrm{~nm}$ to $2.4 \mathrm{~nm}$ were synthesized in the optimized TEFLON $^{\circledR}$ reactor (Figure 38). The size distribution was determined using an empirical equation developed at the University of Arkansas (Equation 9). ${ }^{46}$ 


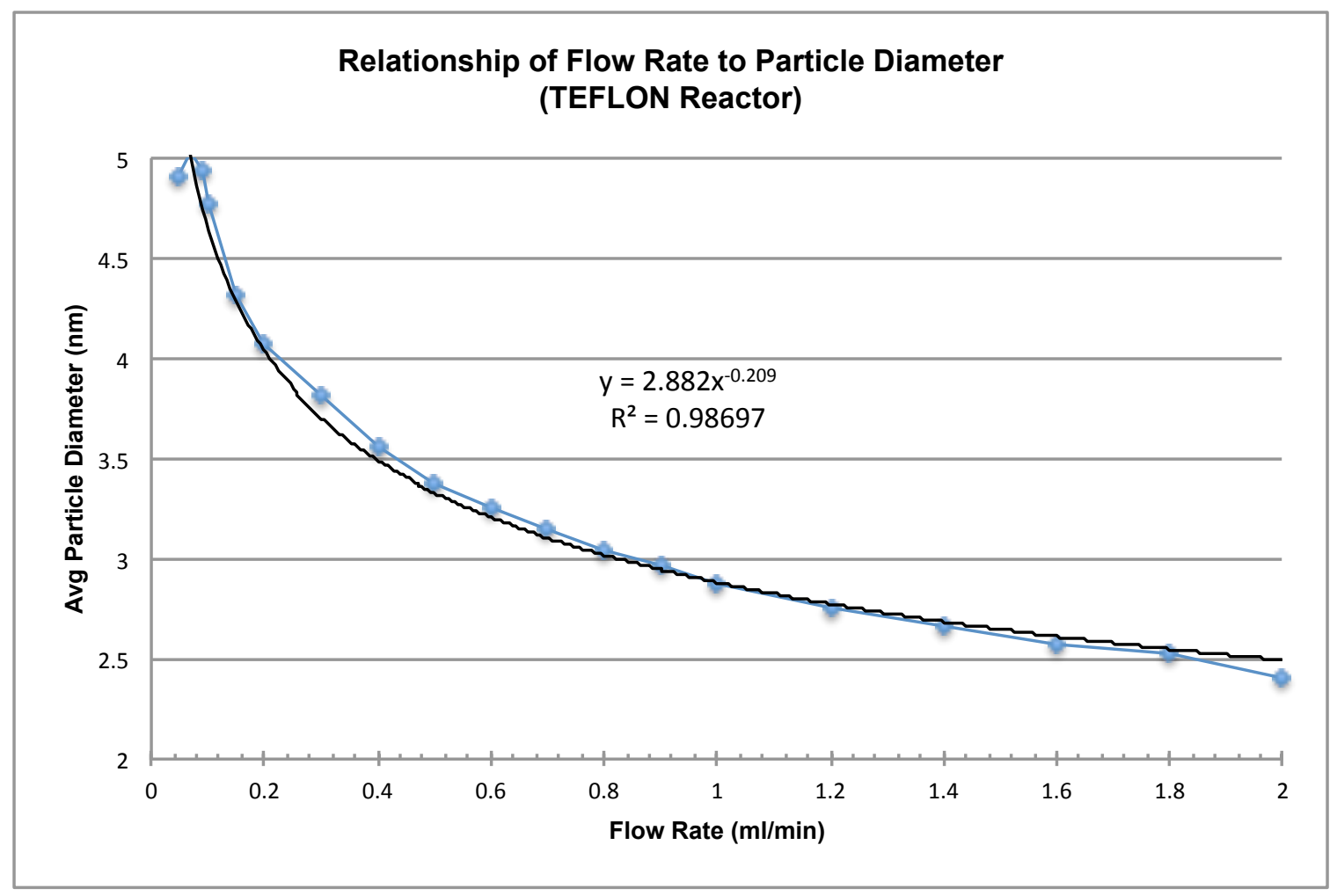

Figure 38: Average particle diameter for each tested flow rate $\left(\right.$ TEFLON $\left.^{\circledR}{ }^{\circledR e a c t o r}\right)$

As shown on Figure 38, the average particle diameter appears to increase with decreasing flow rate.

\subsubsection{Repeatability}

The standard deviation between all experimental trials for the optimized TEFLON ${ }^{\circledR}$ reactor was calculated for the assessed flow rates (Table X). 
Table X: Calculated standard deviation of center wavelength at tested flow rates.

\begin{tabular}{|c|c|}
\hline $\begin{array}{c}\text { Flow Rate } \\
(\mathbf{m l} / \mathbf{m i n})\end{array}$ & $\begin{array}{c}\text { Standard Deviation of Center } \\
\text { Wavelength (nm) }\end{array}$ \\
\hline 0.05 & 4 \\
\hline 0.07 & 6 \\
\hline 0.09 & 5 \\
\hline 0.1 & 5 \\
\hline 0.15 & 7 \\
\hline 0.2 & 6 \\
\hline 0.3 & 4 \\
\hline 0.4 & 4 \\
\hline 0.5 & 4 \\
\hline 0.6 & 5 \\
\hline 0.7 & 5 \\
\hline 0.8 & 6 \\
\hline 0.9 & 6 \\
\hline 1 & 6 \\
\hline 1.2 & 6 \\
\hline 1.4 & 7 \\
\hline 1.6 & 6 \\
\hline 1.8 & 6 \\
\hline 2 & 0 \\
\hline Average StDev & 5 \\
\hline
\end{tabular}

The average standard deviation was $5 \mathrm{~nm}$ and the maximum standard deviation is $7 \mathrm{~nm}$.

The large standard deviations in center wavelength are theorized to stem from the use of octadecene that has either been contaminated or degraded due to age in the first two trials on 5_28_14 and 6_21_14. A table of standard deviation of the center wavelength based on flowrate was created from two new octadecene trials and two old octadecene trials (Table XI). 
Table XI: Optimized TEFLON ${ }^{\circledR}$ reactor comparison of center wavelength standard deviation by flowrate for old and new octadecene solvent

\begin{tabular}{|c|c|c|}
\hline $\begin{array}{c}\text { Flow Rate } \\
(\mathbf{m l} / \mathbf{m i n})\end{array}$ & $\begin{array}{c}\text { STDEV of Center Wavelength } \\
\text { New Octadecene (nm) }\end{array}$ & $\begin{array}{c}\text { STDEV of Center Wavelength } \\
\text { Old Octadecene (nm) }\end{array}$ \\
\hline 0.05 & 1 & 3 \\
\hline 0.07 & 0 & 4 \\
\hline 0.09 & 1 & 3 \\
\hline 0.1 & 0 & 3 \\
\hline 0.15 & 1 & 2 \\
\hline 0.2 & 0 & 1 \\
\hline 0.3 & 0 & 3 \\
\hline 0.4 & 1 & 4 \\
\hline 0.5 & 1 & 5 \\
\hline 0.6 & 1 & 6 \\
\hline 0.7 & 0 & 7 \\
\hline 0.8 & 1 & 7 \\
\hline 0.9 & 1 & 6 \\
\hline 1 & 1 & 6 \\
\hline 1.2 & 0 & 8 \\
\hline 1.4 & 1 & 7 \\
\hline 1.6 & 0 & 7 \\
\hline 1.8 & 0 & 5 \\
\hline 2 & 0 & 5 \\
\hline Average & 1 & \\
\hline
\end{tabular}

The average standard deviation for the tests using new octadecene is $1 \mathrm{~nm}$ and the maximum standard deviation is $1 \mathrm{~nm}$. A much larger center wavelength standard deviation occurs in the two old octadecene trials $(5 \mathrm{~nm})$. Using new octadecene is thought to increase process reliability, however, more testing is needed to substantiate this theory.

\subsection{Comparison of Repeatability Between the Optimized TEFLON ${ }^{\circledR}$ Reactor and Optimized 316 Stainless Steel Reactor}

Comparisons between the optimized TEFLON ${ }^{\circledR}$ reactor and optimized 316 stainless steel reactor can be drawn from the old octadecene tests. But again as stated earlier the small 
sample size for the old octadecene TEFLON ${ }^{\circledR}$ reactor trials decreases statistical significance. More trials with new octadecene are required for comparisons to be statistically significant. That being said, the stainless steel reactor with old octadecene (AVG STDEV 3nm) appears to have better process repeatability in obtaining consistent center wavelength values given flow rate than the TEFLON ${ }^{\circledR}$ reactor with old octadecene (AVG STDEV 5nm). 


\section{DISCUSSION}

This alternative microfluidic process was found to successfully demonstrate a correlation between the flow rate and the fluorescence profile of quantum dot solution during synthesis. The central peak wavelength was controlled to within a $6 \mathrm{~nm}$ average standard deviation for all tested reactors by varying flow rate.

\subsection{Microfluidic Synthesis Method}

The process can produce quantities of solution ranging from $1 \mathrm{ml}$ to $50 \mathrm{ml}$ per trial for any desired flow rate. The incorporation of microfluidics allows for better control of the parameters affecting nucleation and growth. The incorporation of this new synthesis method and analysis technique improves the manufacturability of quantum dots produced at Cal Poly San Luis Obispo, allowing for repeatability and greater precision.

\subsection{Microfluidic Vs. Bulk Synthesis Process}

A comparison of microfluidic synthesized quantum dots and conventional bulk synthesized quantum dots was performed using the fluorescence flow cell. The large thermal gradients in the bulk synthesis method create significantly larger particle size distributions than the microfluidic synthesis method. This is evidenced by a single peak at each given flow rate for the microfluidic reactor rather than the wide peaks often seen in the bulk synthesis method. Figure 39 shows two spectral peaks with relatively similar center wavelengths. The blue line represents the spectral peak of quantum dots synthesized with the microfluidic reactor. The red line represents the spectral peak of 
quantum dots synthesized with the bulk synthesis method. The microfluidic synthesis peak is narrower, is more pronounced, and has less noise than the bulk synthesis peak.

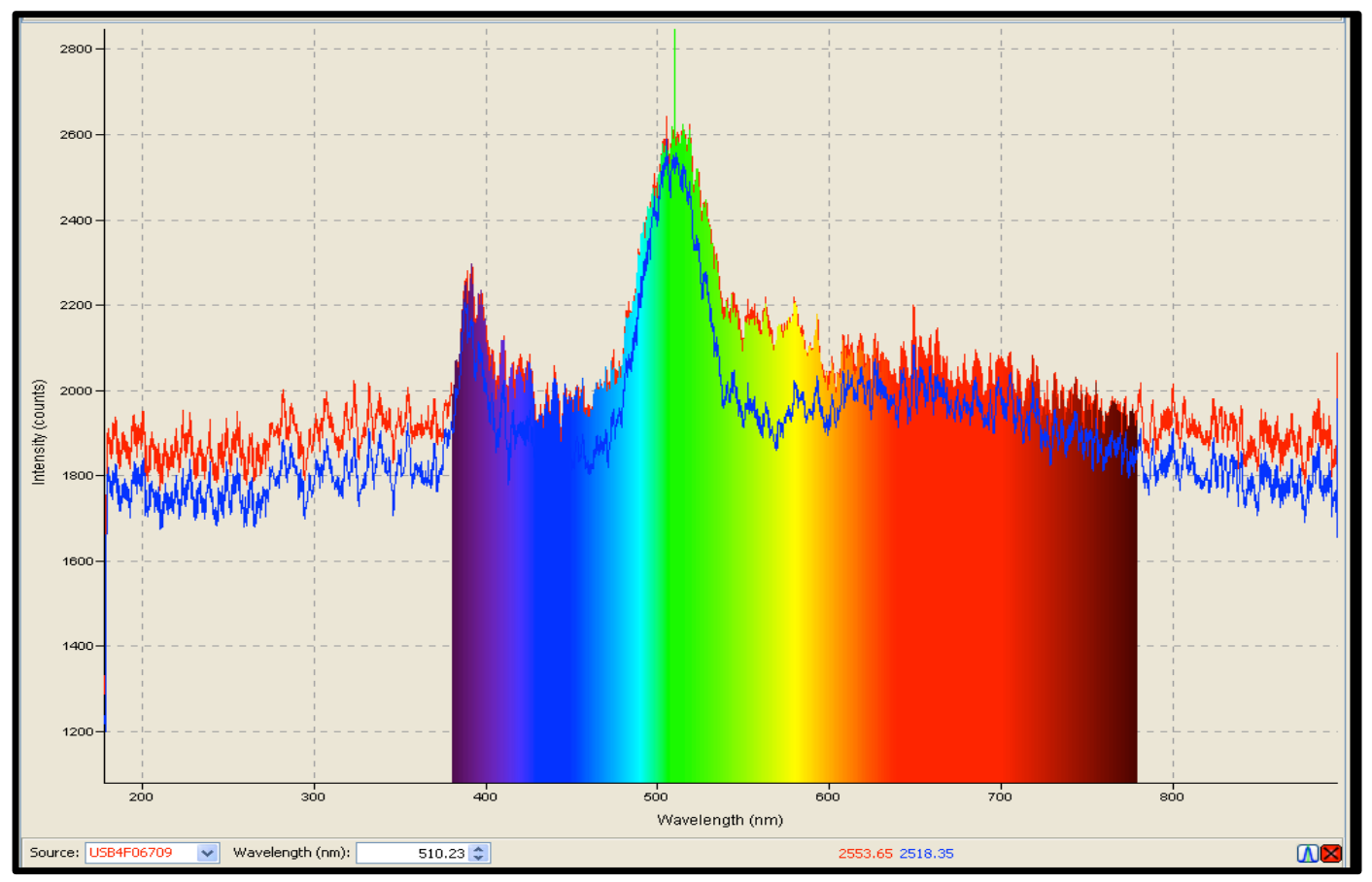

Figure 39: Comparison of microfluidic reactor "blue" to bulk synthesis "red" synthesized quantum dots.

Production of quantum dots of a desired size is possible with microfluidic synthesis by eliminating possibilities of human error during extraction. This is evident in the color spectrum of various samples of quantum dots produced (Figure 40). Many more colors were produced with this method than ever before at Cal Poly. 


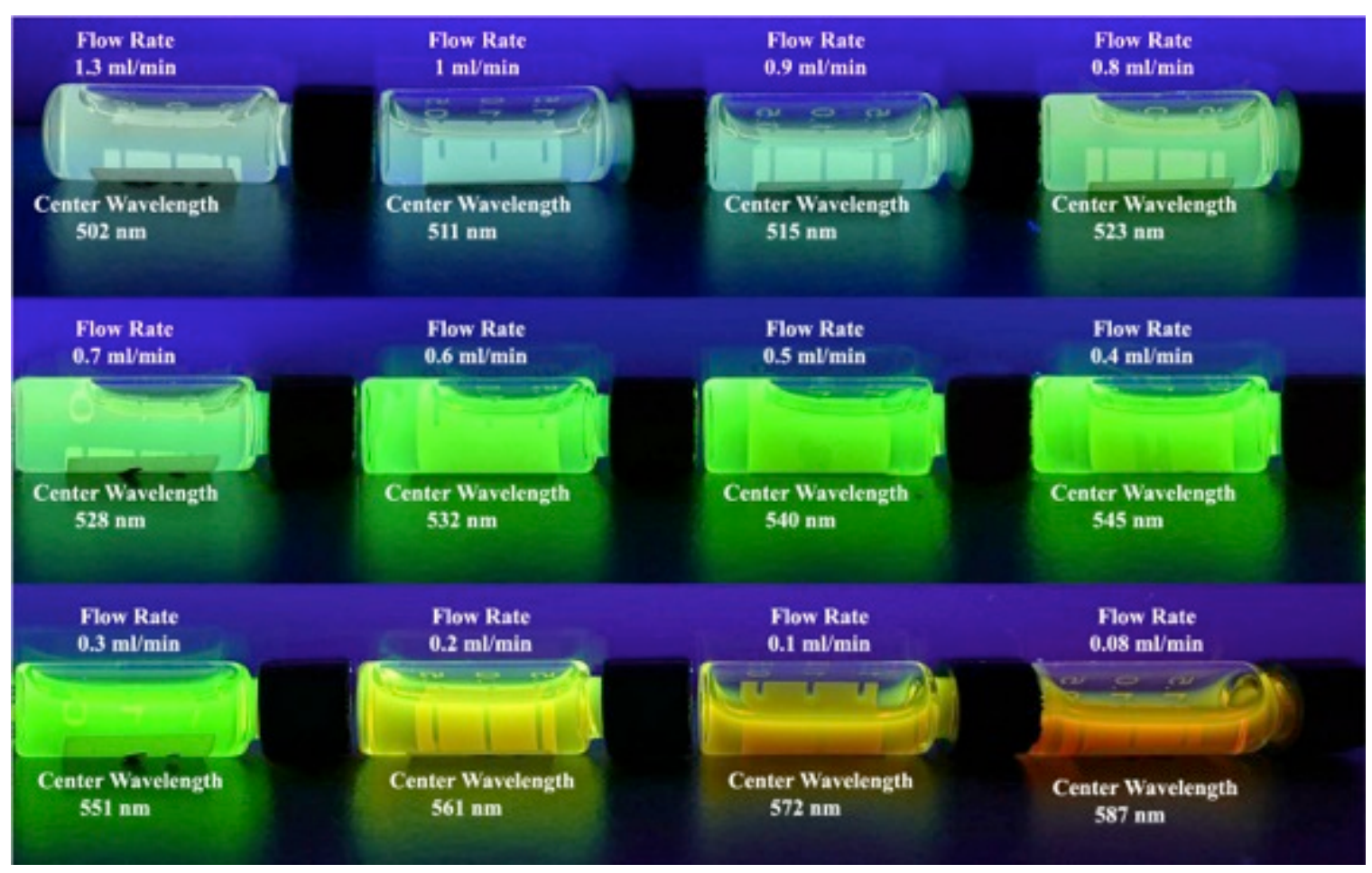

Figure 40: Photograph of every other sample from trial three. Note the relatively uniform shifts in center wavelength with decreasing flow rate.

Moreover, the fluorescence spectrum of the microfluidic quantum dots is slightly higher in intensity. These factors suggest that the outlined microfluidic reactor provides an improved alternative method for quantum dot synthesis at the Cal Poly Nanotechnology Lab.

\subsection{Benefits of Flow Cell Analysis}

The current quantum dot analysis in the Cal Poly Nanotechnology Lab is conducted through the use of cuvette sampling. A cuvette measurement is conducted by shining UV light through the cuvette into a fiber optic cable. The data are then stored and analyzed using a spectrometer in conjunction with SpectraSuite ${ }^{\circledR}$ software. Even though cuvette analysis provides good fluorescence data with high counts, this process can often be messy and time consuming. The utilization of a flow cell for fluid analysis enables real- 
time data analysis. Monitoring of variations between samples was possible because of real-time data analysis and documentation using a fluorescence flow cell in conjunction with a microfluidic reactor. Real-time analysis also accounts for tuning reaction parameters such as the temperature of the silicon oil reactor bath. When analyzing microliter-sized flows, a detailed analysis is conducted while minimizing sample volumes and waste. Because the quantum dots flow through the flow cell, there is no need to transport samples between synthesis and analysis. The possibility of spills or contamination that occur during cuvette analysis is eliminated.

\subsection{Repeatability of Proof of Concept Reactor}

The large standard deviations at the $0.1 \mathrm{ml} / \mathrm{min}, 0.2 \mathrm{ml} / \mathrm{min}$, and $0.8 \mathrm{ml} / \mathrm{min}$ flow rates were most likely caused by fluctuations in synthesis temperature (Table IV). In an experimental trial at $250^{\circ} \mathrm{C}$ a shift of approximately $8 \mathrm{~nm}$ for all flow rates was noted. Assuming a linear relationship, this infers nominal shift of center wavelength of $0.53 \mathrm{~nm} /{ }^{\circ} \mathrm{C}$. The hotplate used in the synthesis procedure does not self-regulate temperature, and human error caused fluctuations in the oil bath temperature of $\pm 5^{\circ} \mathrm{C}$ at these flow rates. Literature describes controlling Cd-Se nanoparticle size by changing the ratio of $\mathrm{Cd}$ and Se precursors. ${ }^{49}$ Consequentially, these fluctuations could also have been caused by differences in concentrations of precursor solutions between trials, however, the differences were not noted for all flow rates. No testing was done on the accuracy of the syringe pump at low flow rates, however, the lowest flow rate showed little deviation between trials. The larger deviations at $0.1 \mathrm{ml} / \mathrm{min}$ and $0.2 \mathrm{ml} / \mathrm{min}$ were most likely caused by temperature fluctuations of the oil bath. 


\subsection{Repeatability of Optimized Reactors}

The P.O.C TEFLON ${ }^{\circledR}$ reactor, optimized 316 stainless steel reactor, and optimized TEFLON $^{\circledR}$ reactor all had average standard deviations of center wavelength across all flow rates below $6 \mathrm{~nm}$ (Table XII).

Table XII: Average standard deviation across all flow rates for all three tested reactors.

\begin{tabular}{|l|c|}
\hline \multicolumn{1}{|c|}{ Reactor } & $\begin{array}{c}\text { Average STDEV (nm) across all flow } \\
\text { rates }\end{array}$ \\
\hline Proof of Concept Reactor & 3 \\
\hline Optimized 316 Stainless Steel Reactor & 3 \\
\hline Optimized TEFLON ${ }^{\circledR}$ Reactor & 5 \\
\hline
\end{tabular}

Excluding the botched trials discussed in the optimized 316 stainless steel reactor results section, the average standard deviation in center wavelength for the 316 stainless steel reactor coil was $3 \mathrm{~nm}$.

The residence time should have been the same between the optimized TEFLON ${ }^{\circledR}$ reactor and the optimized 316 stainless steel reactor; however, oxide growth in the stainless steel reactor is thought to have reduced the stainless steel reactor's inner diameter. This both increased flow velocity through the reactor tubing and decreased residence time. This theory is supported by the fact that the TEFLON ${ }^{\circledR}$ reactor created larger quantum dots than the stainless steel reactor at the same flow rates (Figure 41). 


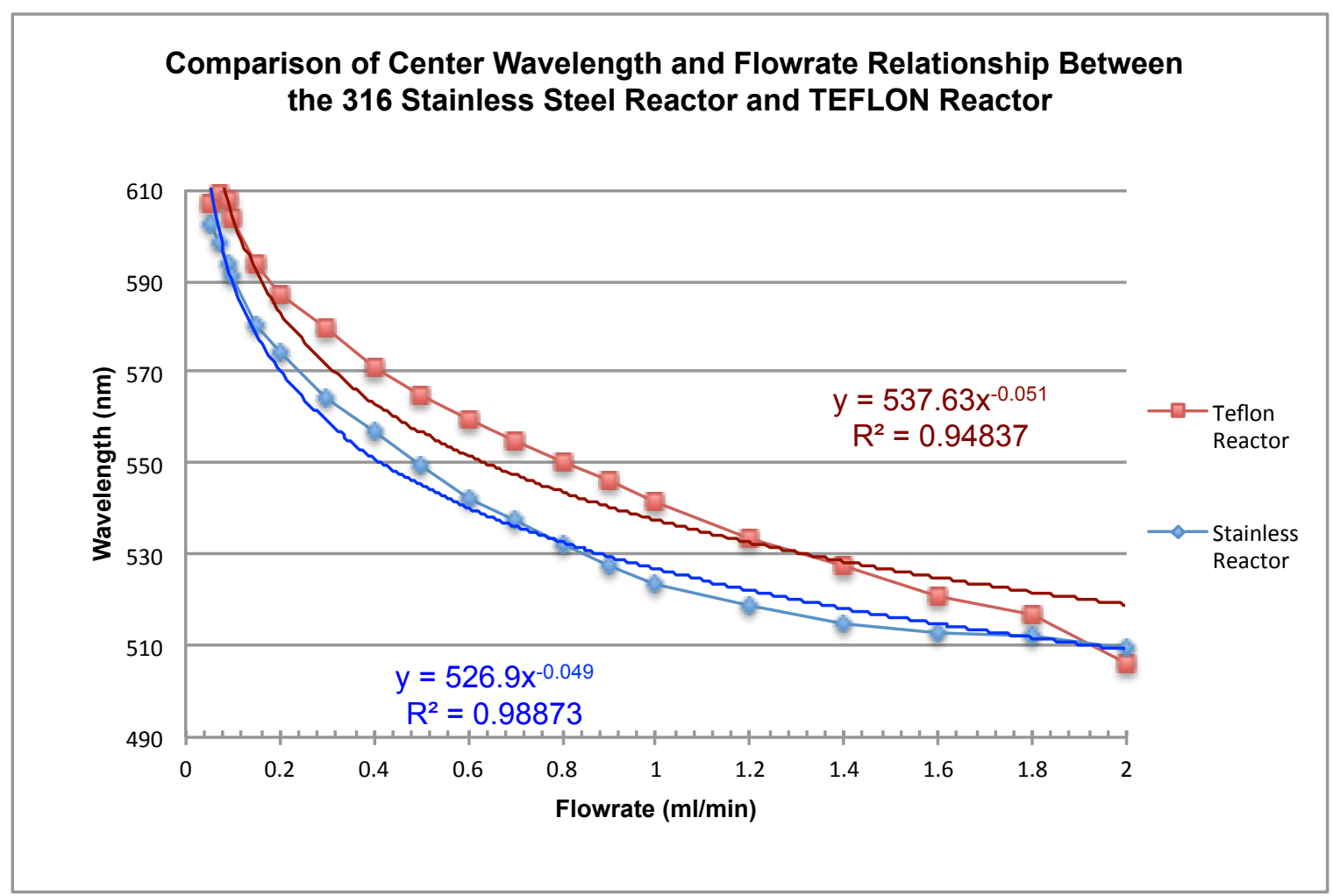

Figure 41: Comparison of center wavelength and flowrate relationship between the optimized 316 stainless steel reactor and optimized TEFLON ${ }^{\circledR}$ reactor. Note that the TEFLON ${ }^{\circledR}$ reactor produces larger quantum dots with a higher center wavelength than the stainless steel reactor at the same flow rate.

The first two optimized TEFLON ${ }^{\circledR}$ reactor trials differ substantially from the last two trials causing the standard deviation to be significantly larger at $5 \mathrm{~nm}$. The only apparent difference in the processing between these trial sets was the use of a new bottle of bottle of octadecene. Two subsequent trials were conducted in the optimized TEFLON ${ }^{\circledR}$ reactor using the new octadecene. Quantum dots fabricated with the old octadecene grow larger with an average increase in center wavelength of 8nm (Figure 42). 


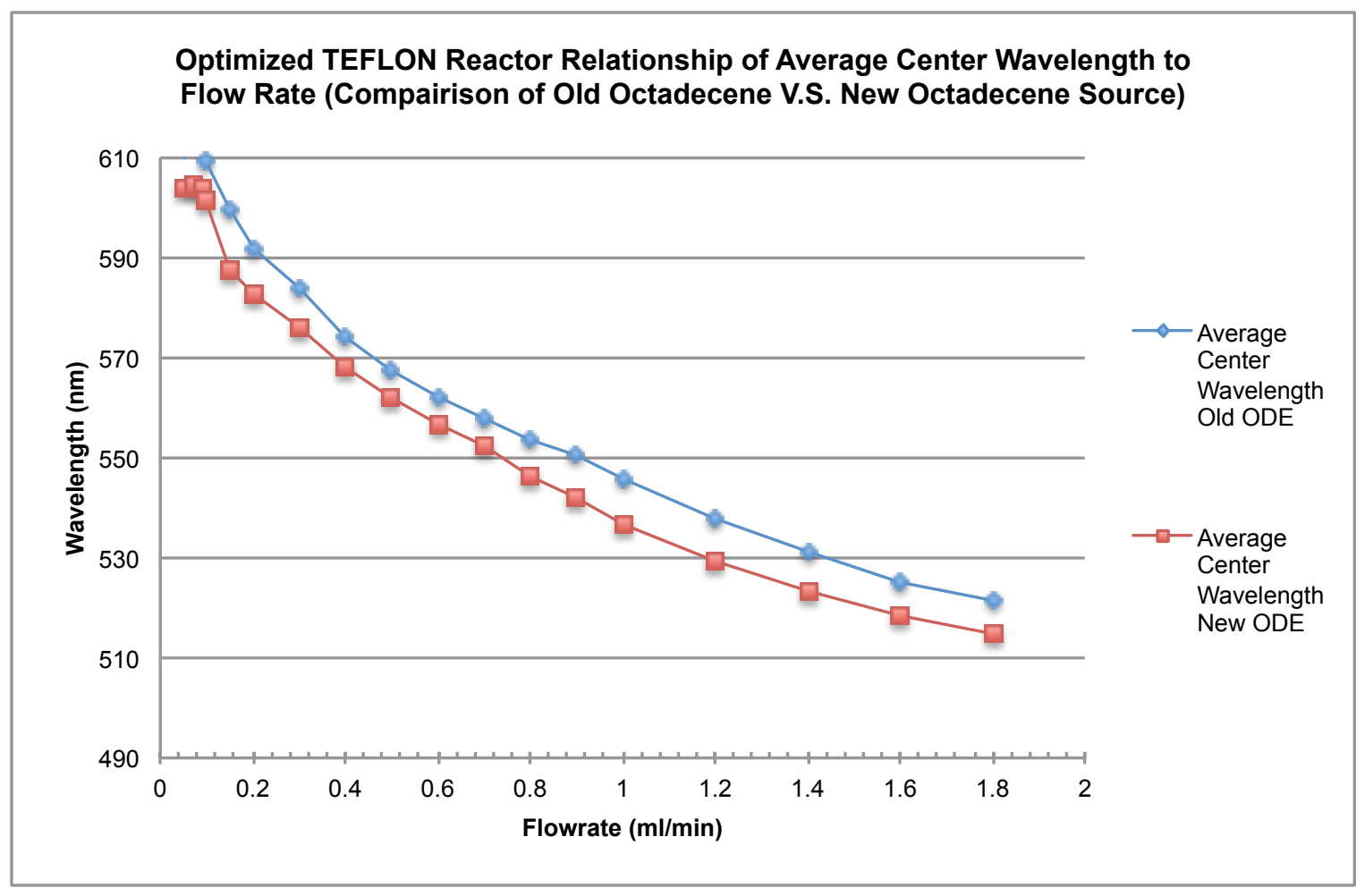

Figure 42: Comparison of old source of octadecene to new source of octadecene

Contamination or chemical degradation of the old source of octadecene is likely the cause of this difference. The old bottle of octadecene was 5 years old at the time of use. The average standard deviation in center wavelength between the two trials for the old octadecene was $5 \mathrm{~nm}$. The average standard deviation in center wavelength between the two trials for the new octadecene was $1 \mathrm{~nm}$. Assuming that the process conditions were the same for both sets of trials the standard deviations resulting from the use of new octadecene are significantly lower. While the new octadecene indicates better synthesis process reliability, the differences between octadecene sources expose error in the process characterization and derived equations as the old octadecene was used in the majority of characterization. 


\subsection{Investigation of Factors Causing Nanocrystal Polydispersity}

When the nucleation and growth reaction begins during QD synthesis there is a high concentration of reaction monomers in solution. ${ }^{50}$ The critical radius is relatively small and the high degree of supersaturation drives a burst of nucleation. The nucleation reaction proceeds more quickly as degree of supersaturation increases. ${ }^{51}$ Particles that nucleate right away have a large concentration of reaction monomers in solution and are able to grow unconstrained by lack of monomers. It follows that particles that nucleate later in this initial nucleation burst have less time to grow. This leads to polydispersity during the initial nucleation burst. Following the initial nucleation burst the bulk energy of the solution dips below a critical point required to form new nuclei (2). Here growth continues only on existing nuclei. The concentration of reaction monomers is still high and particle growth occurs fastest on particles with a radius double the critical radius. ${ }^{52}$ The statistical majority of nucleates are larger than the critical radius and small nuclei close to double the critical radius grow more quickly. This leads to a narrowing of polydispersity (focusing regime). As growth progresses the concentration of reaction monomers decreases and the critical radius increases (Equation 7). The statistical majority of particles exist at the critical radius. Particles larger than the critical radius grow while particles smaller than the critical radius shrink causing widening of the particle size distribution (broading regime) ${ }^{53}$. Depending on residence time the nucleation and growth reaction can be terminated in the various stages outlined above. If the residence time is too short, the sample will contain quantum dots with a large size distribution because the sample has not had sufficient time in the focusing regime to 
minimize polydispersity inherent in the nucleation burst. If the residence time is too long broadening of the particle size distribution occurs due to Ostwald ripening.

\subsubsection{Laminar Flow Contribution to Nanocrystal Polydispersity}

Under laminar flow conditions, the precursor solution does not mix significantly within the capillary tubing. This means that reagent will remain in the same relative position, with the exception of diffusion, for the duration of the reaction. For example reagent that enters the reactor near the reactor wall will remain near the wall until it exits the reactor. By itself this will have no effect on the quantum dot size distribution, however, if reaction parameters are non-uniform throughout the reactor the lack of mixing will exacerbate any disparities in particle size.

\subsubsection{Nucleation Method Contribution to Nanocrystal Polydispersity}

In a microfluidic device exhibiting laminar flow both, heterogeneous nucleation and homogeneous nucleation can occur simultaneously. The liquid that enters the reactor channel mixes only by diffusion. Thus reagent in the center of the channel never touches the exterior walls. Fluid in contact with the exterior walls could possibly nucleate nanocrystals by heterogeneous nucleation whereas fluid in the interior of the channel nucleates nanocrystals through homogeneous nucleation. Nanocrystals that nucleate and grow through heterogeneous nucleation will have less of an energy barrier due to decreased surface energy and thus grow larger than nanocrystals that nucleate through homogeneous nucleation. $^{54}$ 


\subsection{Investigation of Polydispersity at Low Residence Times (Spectral Tail)}

Small quantum dots that fluoresce in the $550-600 \mathrm{~nm}$ range were successfully produced by increasing the flow rate of the reagent solution from $0.3 \mathrm{ml} / \mathrm{min}$ up to $2 \mathrm{ml} / \mathrm{min}$. As the flow rate was increased, a broad tail in the fluorescent profile from $550 \mathrm{~nm}$ to $800 \mathrm{~nm}$ gradually becomes more pronounced (Figure 43)

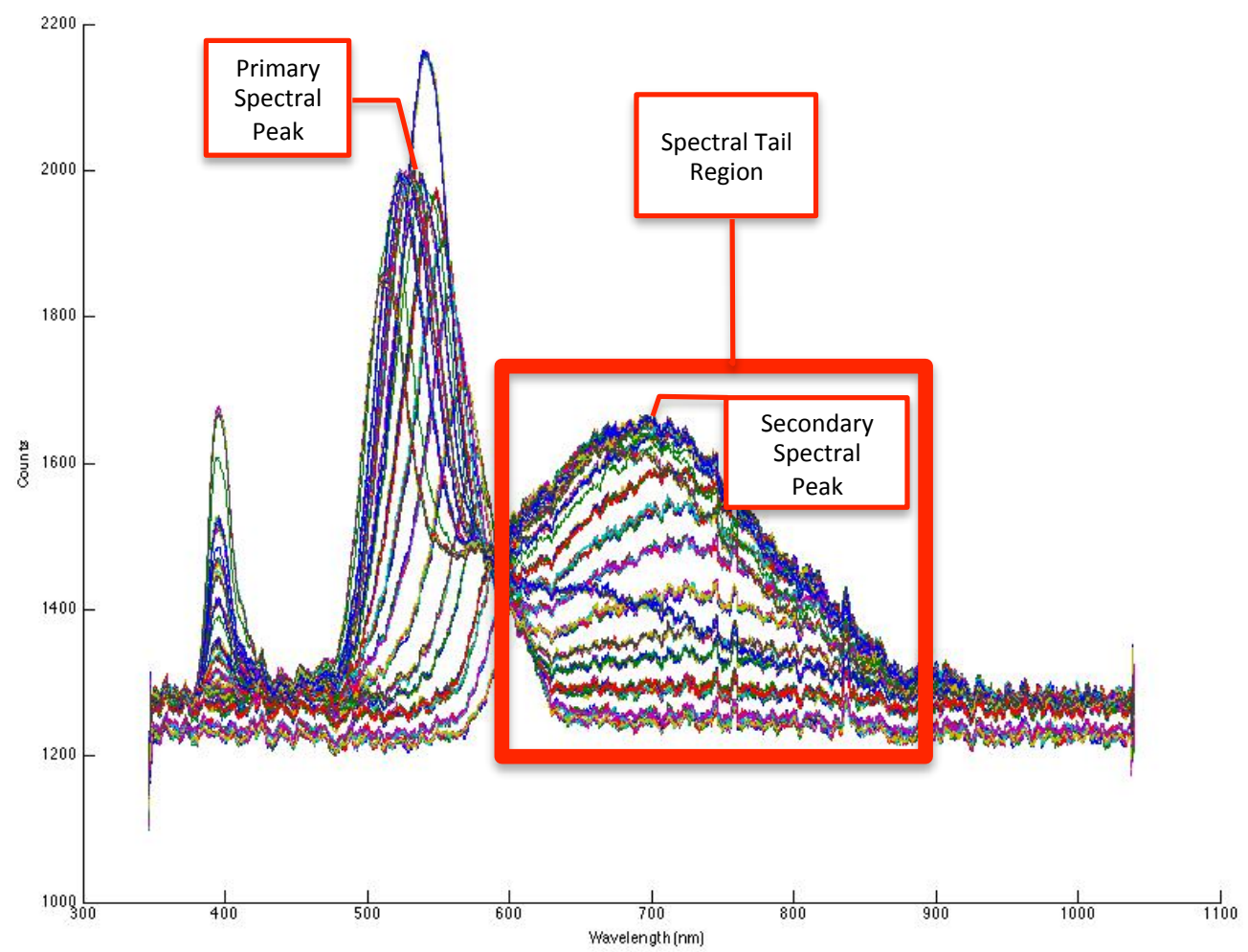

Figure 43: Secondary spectral peaks form a "spectral tail effect" as flow rate increases. The broadening of the spectral profile indicates increasing polydispersity.

This tail can be attributed to Cd-Se polydispersity because fluorescence depends on size. This trend was observed in both of the optimized reactors, however, it was apparent to a greater degree in the 316 stainless steel reactor trials (Appendix C-Appendix E). The tail effect appears to occur only at low residence times, but the tail itself suggests larger 
particles form than high residence times were able to create in this study. Additionally the tail seems to disappear at high residence times as if the large nuclei disintegrate.

\subsubsection{Analysis of the Effects of Thermal Gradients on Nanocrystal Polydispersity}

Both stainless steel and TEFLON ${ }^{\circledR}$ tubing of same length and diameter were used to make reactor coils. Despite this the two reactor coils exhibited different reaction characteristics, the most apparent being a difference in the size and intensity of a spectral tail at high flow rates (low residence times). It was hypothesized that the difference in the two materials thermal properties affected the rate in which heat was transferred to the center of the reactor tubing. This in turn could cause the differences observed in the spectral tail seen at high flow rates. The small dimensions of the reactor coils made experimental testing of thermal effects nearly impossible because thermocouples could not be integrated into the reactor. The thermal transfer of this reaction was modeled theoretically using a finite element analysis physics engine called COMSOL Multiphysics. This physics engine allowed for comparisons of thermal transfer for different flow rates as well as different materials.

\subsubsection{COMSOL Thermal Modeling Procedure}

To obtain a holistic simulation and analysis of physical interactions COMSOL was used to model heat flow from a heat sink through a solid tube enclosing a flowing solution. The heat source i.e. the high temperature reaction bath was set to a constant temperature 
of $240^{\circ} \mathrm{C}$ and the thermal properties for stainless steel and TEFLON ${ }^{\circledR}$ were obtained from literature and can be seen in (Table XIII).

Table XIII: Thermal properties of solid reactor tubing materials used in COMSOL modeling ${ }^{37}$

\begin{tabular}{|c|c|c|}
\hline & $\begin{array}{c}\text { Thermal Conductivity "k" } \\
\left(\mathrm{W} /\left(\mathrm{m}^{*} \mathrm{~K}\right)\right)\end{array}$ & $\begin{array}{c}\text { Specific Heat Capacity "Cp" } \\
\left(\mathrm{J} /\left(\mathrm{kg}^{*} \mathrm{~K}\right)\right)\end{array}$ \\
\hline PTFE (TEFLON $\left.{ }^{\circledR}\right)$ & 0.242 & 970 \\
\hline 316 Stainless Steel & 14 & 490 \\
\hline
\end{tabular}

These properties were input into COMSOL and assigned to a three-dimensional model of the tubing created within COMSOL with dimensions 0.5 -inch long 1/32-inch inner diameter and 1/16-inch outer diameter. Pure octadecene was chosen to model the precursor solution as it represents the majority solvent. Thermal and mechanical properties of octadecene were obtained from literature. The properties of this solvent vary greatly with temperature so equations were generated from empirical data to better model the dynamic system (Equation 13, Equation 14, Equation 15).

Equation 13: Octadecene heat capacity $\left(\mathrm{J} /\left(\mathrm{kg}^{*} \mathrm{~K}\right)\right)^{55}$

$$
\begin{aligned}
& \mathrm{C}_{p}=0.0022\left(T_{(K)}\right)^{2}+1.2588\left(T_{(K)}\right)+1596.3 \\
& \mathrm{R}^{2}=0.99995
\end{aligned}
$$

Equation 14: Octadecene thermal conductivity $(\mathrm{W} /(\mathrm{m} * \mathrm{~K}))^{40,55}$

$$
\begin{aligned}
& \mathrm{k}=-3 \mathrm{E}^{-07}\left(T_{(K)}\right)^{2}+5 \mathrm{E}^{-05}\left(T_{(K)}\right)+0.1464 \\
& \mathrm{R}^{2}=0.99687
\end{aligned}
$$

Equation 15: Octadecene viscosity $\left(\mathrm{Pa}^{*} \mathrm{~s}\right){ }^{40,55}$

$$
\begin{aligned}
& \eta=4 \mathrm{E}^{-07}\left(T_{(K)}\right)^{2}-0.0003\left(T_{(K)}\right)+0.0605 \\
& \mathrm{R}^{2}=0.99453
\end{aligned}
$$


The thermal gradient that forms from when liquid enters the heated reactor was investigated to determine relative distance until equilibrium at flow rates ranging from $0.05 \mathrm{ml} / \mathrm{min}$ to $2 \mathrm{ml} / \mathrm{min}$. After initial testing it was determined that thermal equilibrium for the slower flow rates occurred in a relatively short distance compared to the overall reactor distance. Equilibrium occurs when all precursor solution for a cross section of the tubing is at the same temperature as the heat source. Proportionally, more time would be spent at the equilibrium reaction temperature for slow flow rates indicating a proportionally larger effect on fast flowrates. The fastest flow rate of $2 \mathrm{ml} / \mathrm{min}$ was selected for analysis. The solution flowing through the reactor was modeled by the majority solvent octadecene, entering the reactor at room temperature $298 \mathrm{~K}$.

\subsubsection{COMSOL Thermal Modeling Results}

Heat transfer to the reactant solution can be characterized by the distance fluid flowing through the reactor tubing travels before reaching thermal equilibrium with the heat source. In laminar flow mixing occurs by diffusion so the fluid flowing through the tubing can be treated as a non-mixing stream where heat will be transferred to the center of the tubing the slowest. The distance the fluid must flow along the z-axis before reaching thermal equilibrium was determined using the temperature profile along the centerline of the thermal field i.e. (the center of the tubing) (Figure 44). 


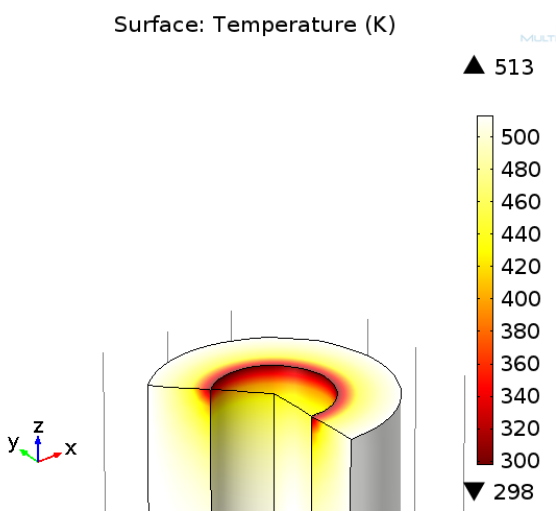

a

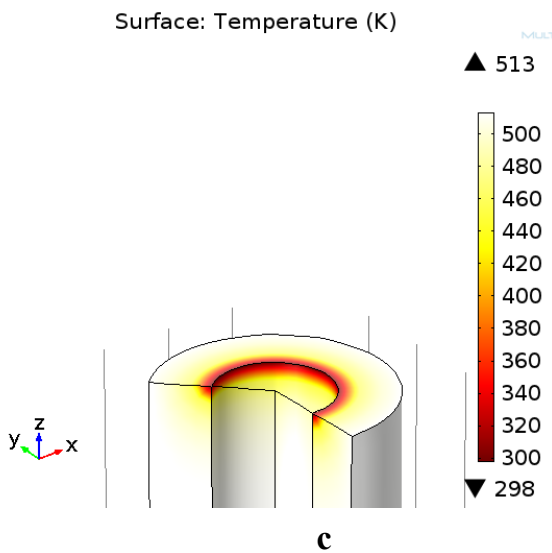

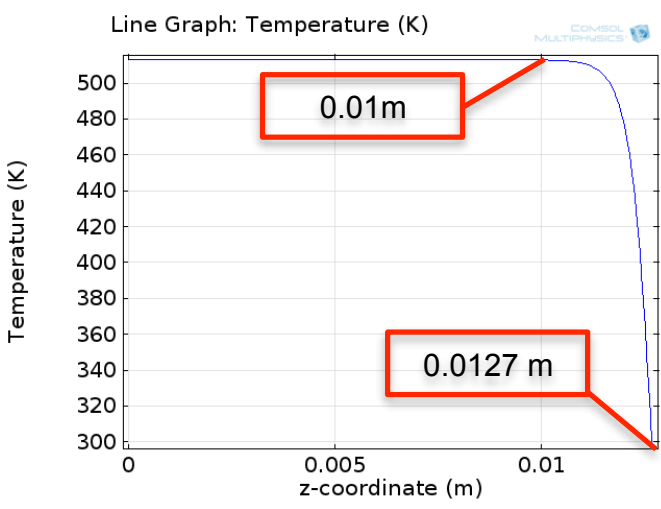

b

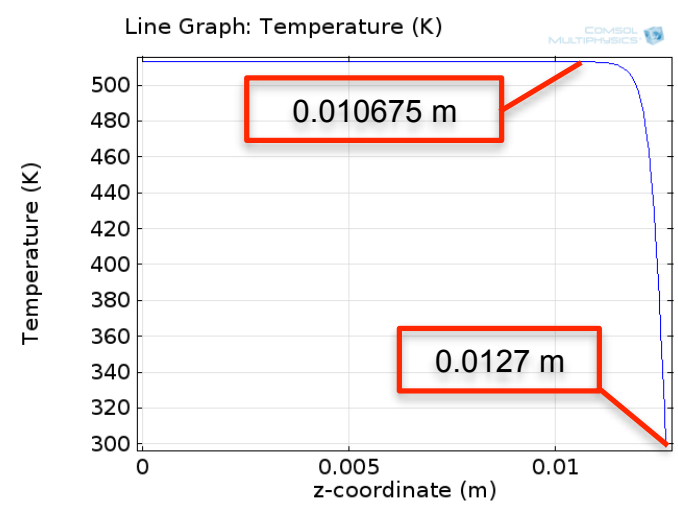

d

Figure 44: Comparison of the inlet thermal gradient for both the optimized TEFLON ${ }^{\circledR}$ and the optimized 316 stainless steel reactors at a flow rate of $2 \mathrm{ml} / \mathrm{min}$. Note fluid injection occurs at a $\mathrm{Z}$ coordinate of $0.0127 \mathrm{~m}$. a) Thermal gradient for TEFLON ${ }^{\circledR}$ reactor tubing. b) Temperature of precursor fluid in the center of the TEFLON ${ }^{\circledR}$ tubing. c) Thermal gradient for 316 stainless steel reactor tubing d) Temperature of precursor fluid in the center of the 316 stainless steel reactor tubing. Note that the $Z$ coordinate axis is backwards, fluid enters at the right at and travels to $0 \mathrm{~m}$.

As expected, the TEFLON ${ }^{\circledR}$ tubing (top right) displays slower transfer of heat to the reactant solution. The fluid has to flow about $1 / 10^{\text {th }}$ of an inch or $0.0027 \mathrm{~m}$ from the injection point before reaching thermal equilibrium. The fluid in the 316 stainless steel tubing (bottom right) has to travel about $2 / 25^{\text {th }}$ of an inch or $0.002 \mathrm{~m}$ from the injection point before reaching thermal equilibrium. 


\subsubsection{Contribution of Fluid Resistance to Nanocrystal Polydispersity}

With continuous flow micro-reactors, the particle size distribution is further broadened by the velocity and residence time distributions inherent to pressure-driven flow. ${ }^{56}$ As the solution flows through the channel little mixing occurs, except through diffusion.

Quantum dots in solution near the exterior of the reactor channel experience a shear force that causes them to move more slowly through the reactor than those on the interior. This effect creates a "slug" where particles closer to the channel walls experience longer residence times and grow larger (red) than the innermost particles (green) (Figure 45). In turn, this causes broadening the particle size distribution.

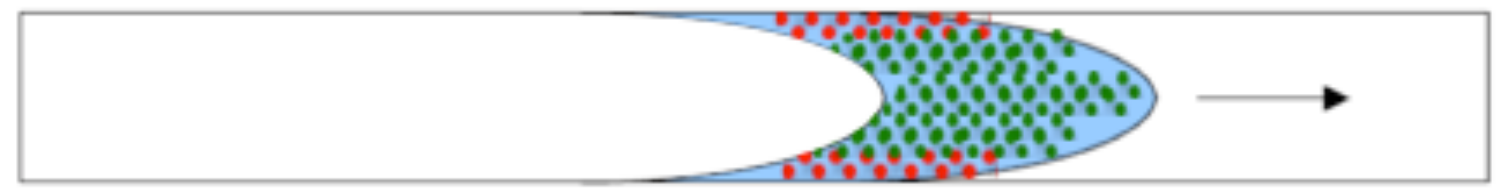

Figure 45: "Slug" resulting from pressure driven flow. Note the slug shape and distribution of quantum dots within the reactor channel.

One possible explanation for the spectral tail is that at short residence times the high concentrations of reaction monomers in solution leads to unconstrained growth for particles trapped next to the channel wall. These particles experience longer residence times and growth is not limited by the concentration of reaction monomers in solution, thus allowing these particles to grow much larger than the average size for that residence time. 


\subsubsection{COMSOL Fluid Velocity Field Modeling}

It is necessary to investigate the difference in residence time the reagent experiences in the interior of the channel compared to the exterior of the channel. COMSOL

Multiphysics was used to model a single-phase flow fluid velocity field for a flow rate of $2 \mathrm{ml} / \mathrm{min}$, the fastest standard flowrate investigated in this study. Octadecene, the majority solvent, was analyzed using single-phase laminar flow to obtain a fluid velocity field (Figure 46).

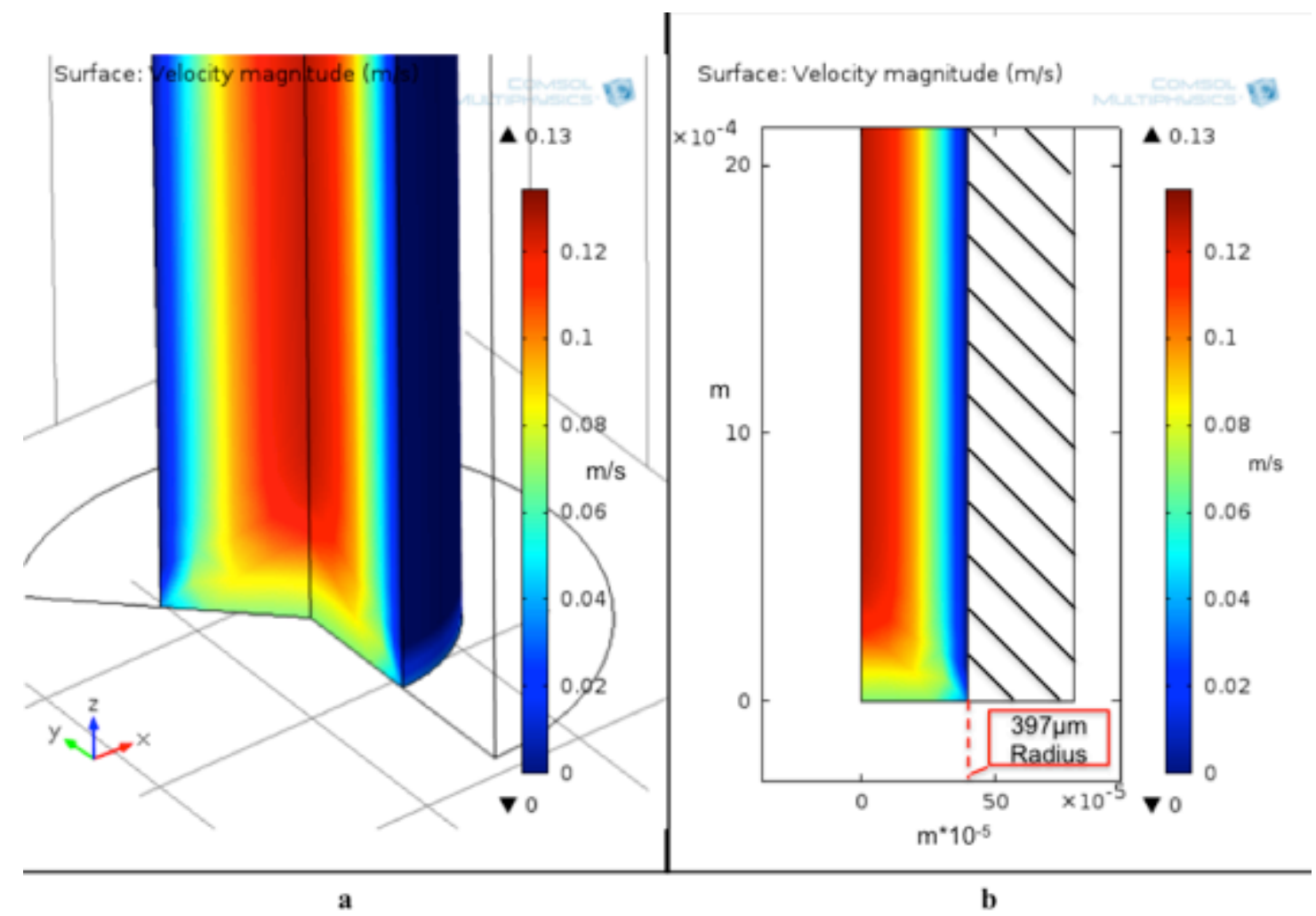

Figure 46: a) Three-dimensional view of the fluid velocity field. Note the wire frame on the outside is the tubing. b) Two-dimensional view of fluid velocity field. Note the radius of the tubing is about $397 \mu \mathrm{m}$. The blue region is about $90 \mu \mathrm{m}$ wide. The green region is about $80 \mu \mathrm{m}$ wide and the red region is about $210 \mu \mathrm{m}$ wide.

The velocity profile on Figure 46 shows the boundary interaction (blue region) between the solvent and the wall of the tubing. The blue region was measured using Image $J$ 
software and is about $90 \mu \mathrm{m}$ wide. Assuming a conservative estimate of velocity of $0.04 \mathrm{~m} / \mathrm{s}$, the residence time for fluid in this region of the reactor is three times longer than the residence time for fluid in the center of the channel traveling at $0.12 \mathrm{~m} / \mathrm{s}$. Despite the slow flow region's relatively low width, it makes up about $40 \%$ of the cross sectional area of the reactor tube.

\subsubsection{Stainless Steel Reactor and the Spectral Tail}

Quantum dot samples synthesized with the stainless steel reactor exhibit a more intense spectral tail across a broader range of wavelengths (Appendix C, Appendix E, Appendix F, Appendix G). This is hypothesized to result from numerous factors including greater surface roughness within the stainless steel reactor $\mathrm{O}_{2}$ contamination, and possible coherent or semi-coherent heterogeneous nucleation.

\subsubsection{Analysis of Reactor Surface Roughness Using SEM}

A Joel SEM was used to analyze the surface roughness of the 316 stainless steel and TEFLON $^{\circledR}$ reactor coils. The 316 stainless steel tubing was sectioned using a Dremel tool and the TEFLON ${ }^{\circledR}$ tubing was sectioned using scissors. The TEFLON ${ }^{\circledR}$ tubing tended to deform during cutting and the channel walls became distorted, however, this was not of concern as the interior surface roughness was the focus of this test (Figure 47). 

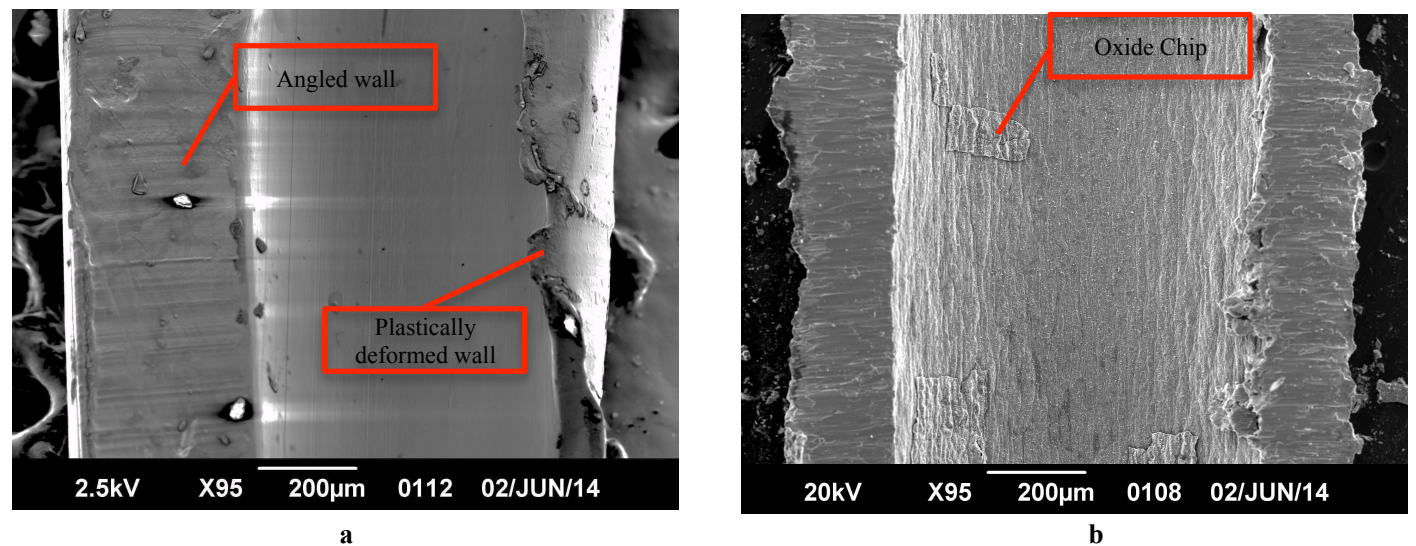

Figure 47: a) SEM micrograph of the optimized TEFLON ${ }^{\circledR}$ reactor tubing at 95X magnification. (Note the smooth surface). b) SEM micrograph of the optimized 316 stainless steel reactor tubing at 95X magnification. (Note the oxide spalling off the sample surface)

Both samples were mounted on a SEM post with a conductive adhesive disk. The samples were then sputtered with gold. Sputtering allows for better resolution under the SEM as the thin conductive layer wicks charge buildup to the grounded sample stage eliminating bright spots. Conductive copper tape was used to connect the grounded sample stage to this thin gold layer. The full procedure for SEM analysis can be found in Appendix G.

\subsubsection{Surface Roughness Results}

The surface roughness of the optimized TEFLON ${ }^{\circledR}$ reactor coil and the optimized 316 stainless steel reactor coil were investigated using Gwyddion image analysis software. Gwyddion allows for the construction of an arbitrary surface roughness indicator using contrast between high and low areas in an SEM micrograph. The parameters for this calculation can be seen in Appendix I. The root mean square RMS (SQ) value was compared between the four micrographs shown on Figure 48. 

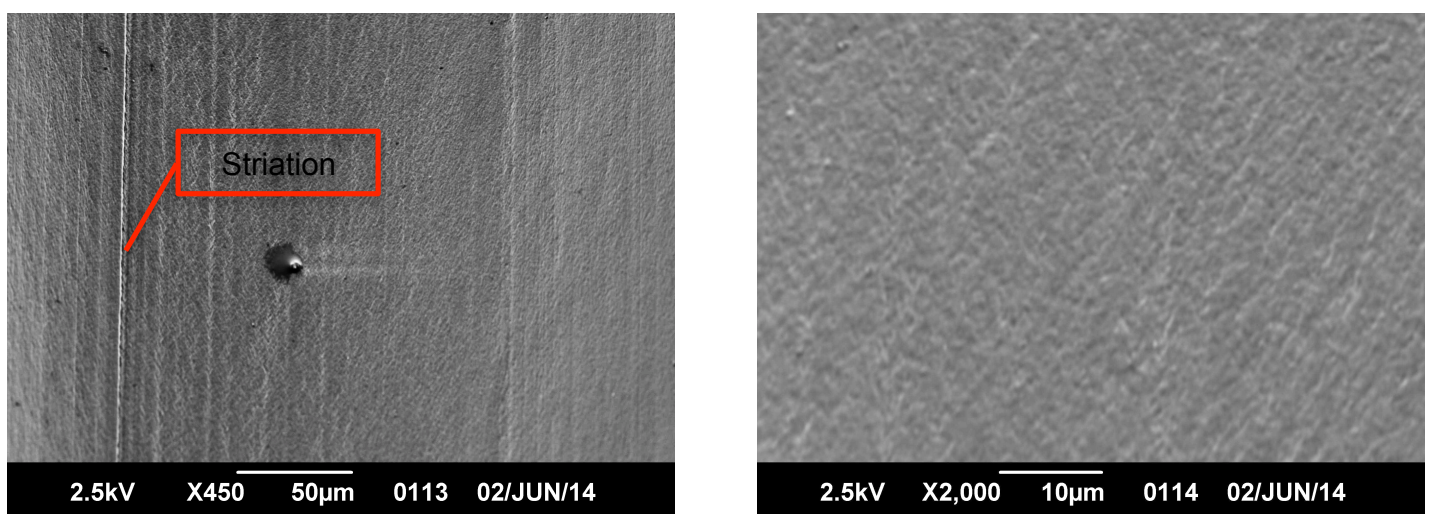

a

b
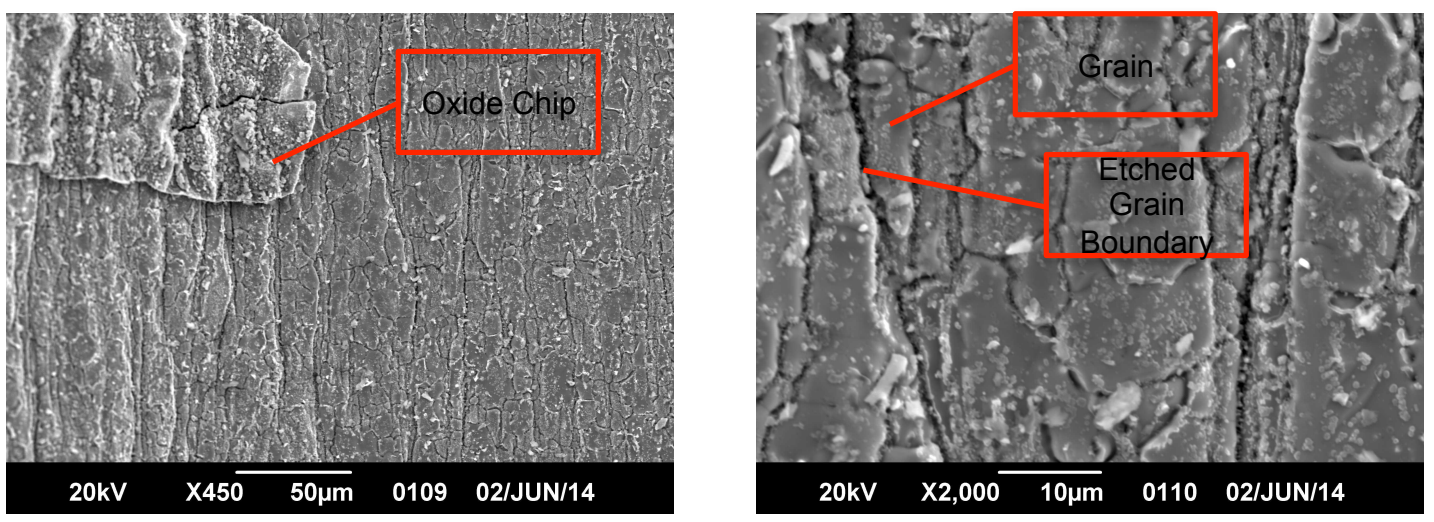

c

d

Figure 48: a) Micrograph of TEFLON ${ }^{\circledR}$ tubing 450X magnification. Note striations formed during extrusion. b) Micrograph of TEFLON ${ }^{\circledR}$ tubing 2000X magnification. c) Micrograph of 316 stainless steel reactor tubing with an oxide chip that has begun to crack and spall off the tubing surface. d) Micrograph of 316 stainless steel reactor tubing showing preferential etching along grain boundaries.

These values can be seen in Table XIV. An oxide formed on the surface of the stainless steel reactor and appears to have spalled off leaving flakes still adhered to the tubing wall. Additional surface roughness appears to be caused by etching of the grain boundaries of the stainless steel sample.

Table XIV: Surface roughness values for the optimized 316 stainless steel reactor and TEFLON ${ }^{\circledR}$ reactor

\begin{tabular}{|c|c|}
\hline Image & Arbitrary Surface Roughness RMS (Sq) \\
\hline a) TEFLON ${ }^{\circledR}$ REACTOR 450X & 0.05 \\
\hline 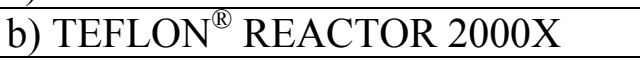 & 0.04 \\
\hline c) 316 Stainless Steel Reactor $450 \mathrm{X}$ & 0.15 \\
\hline d) 316 Stainless Steel Reactor 2000X & 0.13 \\
\hline
\end{tabular}


The TEFLON ${ }^{\circledR}$ reactor appears to be almost 3 times smoother.

\subsubsection{Surface Roughness Discussion}

The increased surface roughness of the optimized 316 stainless steel tubing could affect the liquid solid interface, mixing characteristics, and nucleation method. Oxide chips and etched grains could act as barriers for fluid moving along the wall of the channel in the shear layer, slowing flow, increasing residence time, and possibly increasing the width of the shear layer. Additionally, if these defects are large enough, they potentially could divert enough flow to increase the rate of diffusive mixing by "folding" fluid from the outer edge of the channel into the center. Though unlikely, the oxide could spall off but remain partially attached to the channel wall to create geometries akin to microfluidic mixers. Greater surface roughness correlates to larger surface area. The amount of heterogeneous nucleation occurring on the channel walls might increase as a result. Nucleates might become trapped as flow velocity in the etched grain crevices might not be high enough to knock heterogeneous precipitates off the reactor wall.

\subsection{Physical Characterization of Quantum Dot Size Distribution}

An atomic force microscope (AFM) was used to accurately measure the Cd-Se quantum dots synthesized with the microfluidic reactor and determine the efficacy of the empirical equations relating center wavelength to average quantum dot size. A sample suspension of quantum dots synthesized with the optimized TEFLON ${ }^{\circledR}$ reactor with a florescent peak at $620 \mathrm{~nm}$ was re-suspended in toluene from octadecene, diluted, transferred to a sample surface, and measured. The procedures involved can be seen in Appendix J. 


\subsubsection{Fat Finger Syndrome}

Lateral measurements were not a good representation of quantum dot size in this analysis because of lateral distortion caused by "fat finger syndrome". When the size of the tip approaches the size of the sample, despite the tips near atomic sharpness, the sides of the tip interact with the sample as shown on Figure 49.

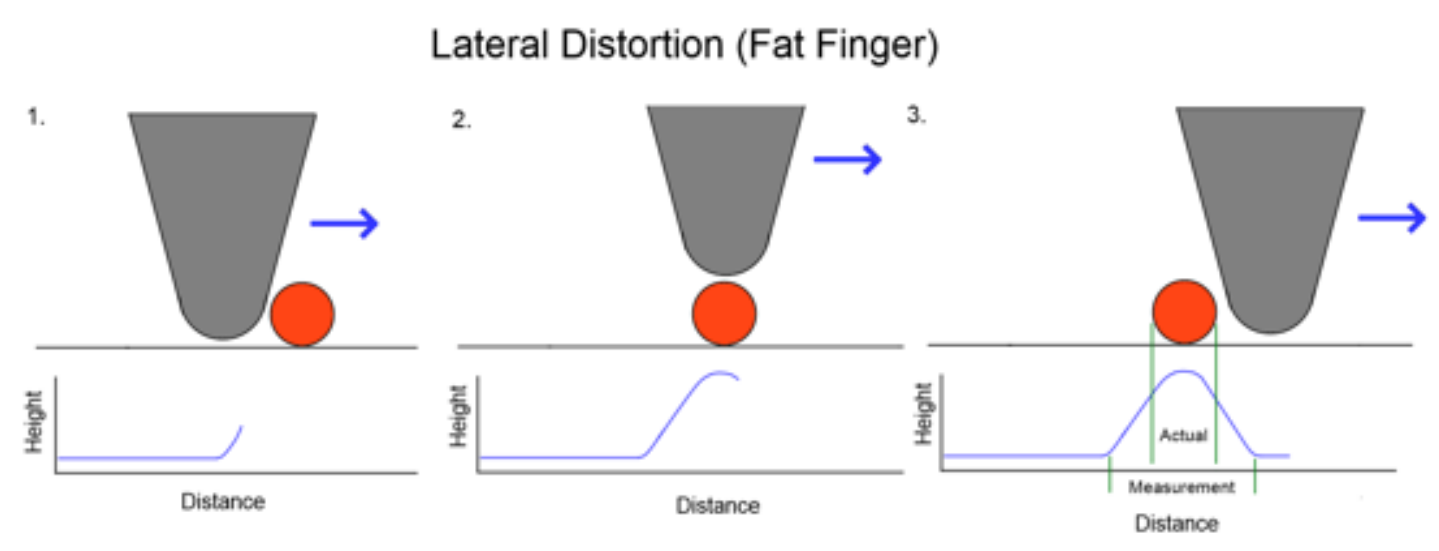

Figure 49: As the AFM tip approaches a quantum dot the side of the tip interacts with the quantum dot (1). The tip measures the apex of the quantum dot (2). The measured profile is distorted because of the interactions of the side of the tip and the quantum dot (3). (R. Gregoriev, personal communication, June 18, 2014)

Features become drawn out and more rounded and topographical measurements cannot be used to accurately access QD size. Instead height change was used.

\subsubsection{AFM Results and Discussion}

A solution of quantum dots with a fluorescence peak at $620 \mathrm{~nm}$ was successfully resuspended in toluene. The toluene quantum dot solution was transferred to a 1 -inch silicon wafer and imaged. Quantum dots ranging from $1.5 \mathrm{~nm}$ to $12 \mathrm{~nm}$ were measured indicating significant polydispersity or agglomeration of nanoparticles (Figure 50). 


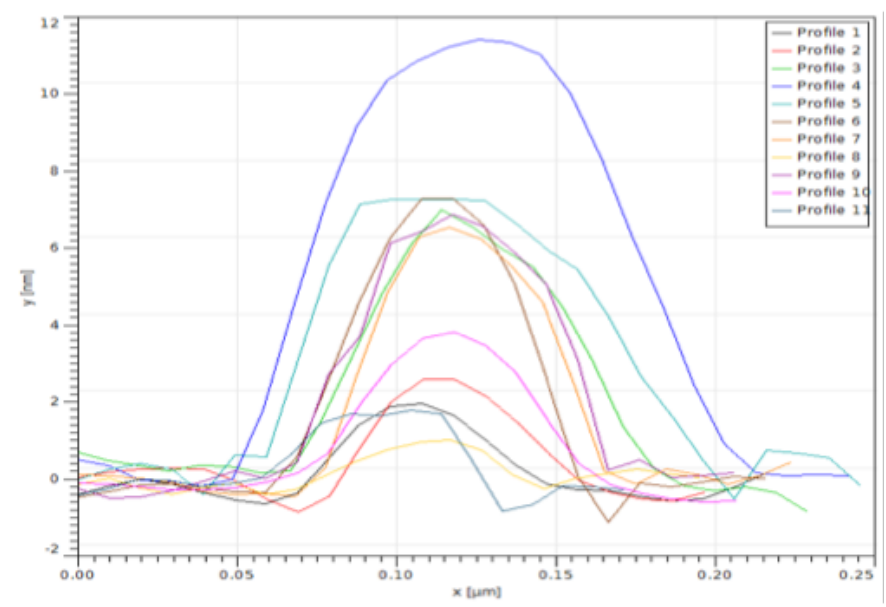

$\mathbf{a}$

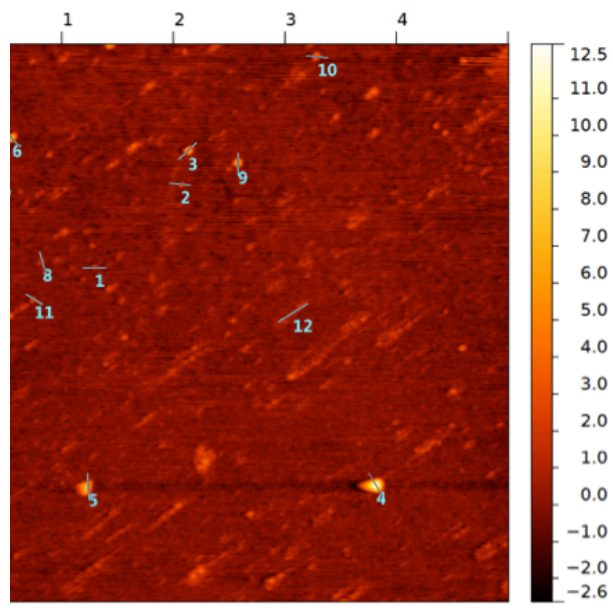

b

Figure 50: Asylum AFM quantum dot measurements on 1" silicon wafer. a) Height profile of 11 scans. b) Micrograph of scan area. Note sizes range from about $1.5 \mathrm{~nm}$ to $12 \mathrm{~nm}$. (R. Gregoriev, personal communication, June 18, 2014)

The center wavelength, $620 \mathrm{~nm}$, should correlate to a marginal size of $5.6 \mathrm{~nm}$ (Equation 9). Five peaks reside at a height of $6 \mathrm{~nm}$, relatively close to the predicted size of $5.6 \mathrm{~nm}$.

Streaking is potentially caused by the directionality of deposition, inherent in the atomization spraying process (Figure $50 \mathrm{~b}$ ). A baseline surface roughness scan was recorded before adding atomized quantum dots to the sample surface (Figure 51). 


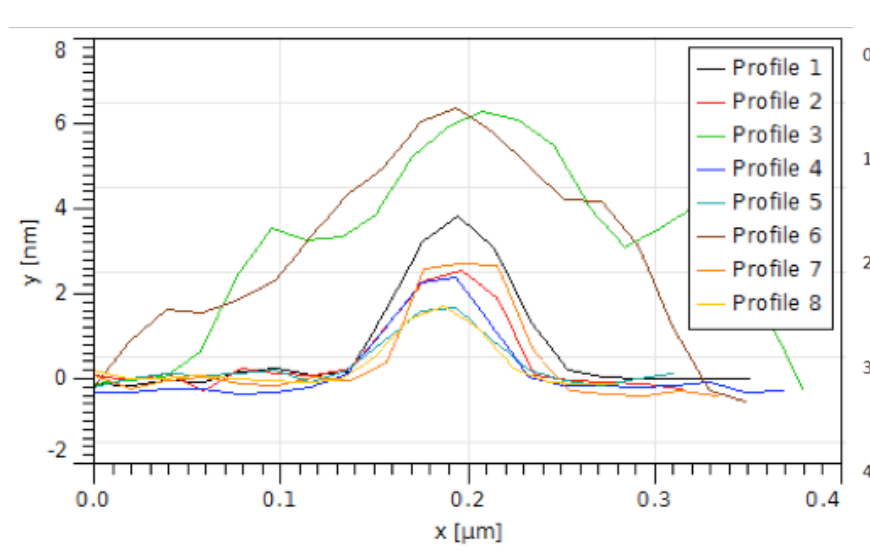

a

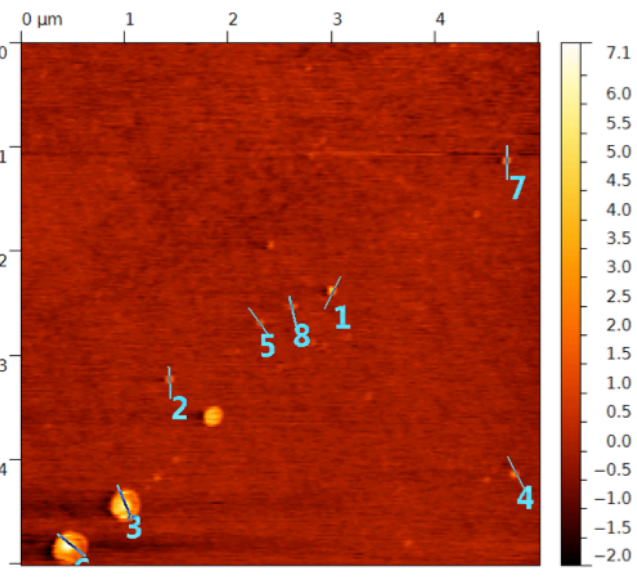

$\mathbf{b}$

Figure 51: Asylum AFM baseline sample surface roughness analysis. Note residual quantum dots after cleaning. a) Height profile of 8 scans. b) Micrograph of scan area. Note sizes range from about 1.8nm to 6nm. (R. Gregoriev, personal communication, June 18, 2014)

Some quantum dots remained adhered to the sample surface and were in the size range of 1.8-6nm. Larger quantum dots and streaking from the atomizer appeared to have been removed by cleaning.

\subsubsection{AFM Conclusion}

Cd-Se Quantum dots ranging in size from $1.5 \mathrm{~nm}$ to $12 \mathrm{~nm}$ were successfully measured using an AFM. Fat finger syndrome effects on lateral resolution made identification of coagulation impossible however, the larger $12 \mathrm{~nm}$ quantum dots are thought to be coagulants. The median size of the measured quantum dots was $6 \mathrm{~nm}$. These quantum dots are within the predicted size range for this synthesis method. 


\subsection{Relationship of Nucleation and Growth Theory to Microfluidic Reactor}

\section{Results}

To compare the trends predicted by nucleation and growth theory to the results of this microfluidic synthesis method a plot relating average quantum dot size to average residence time was created (Figure 52).

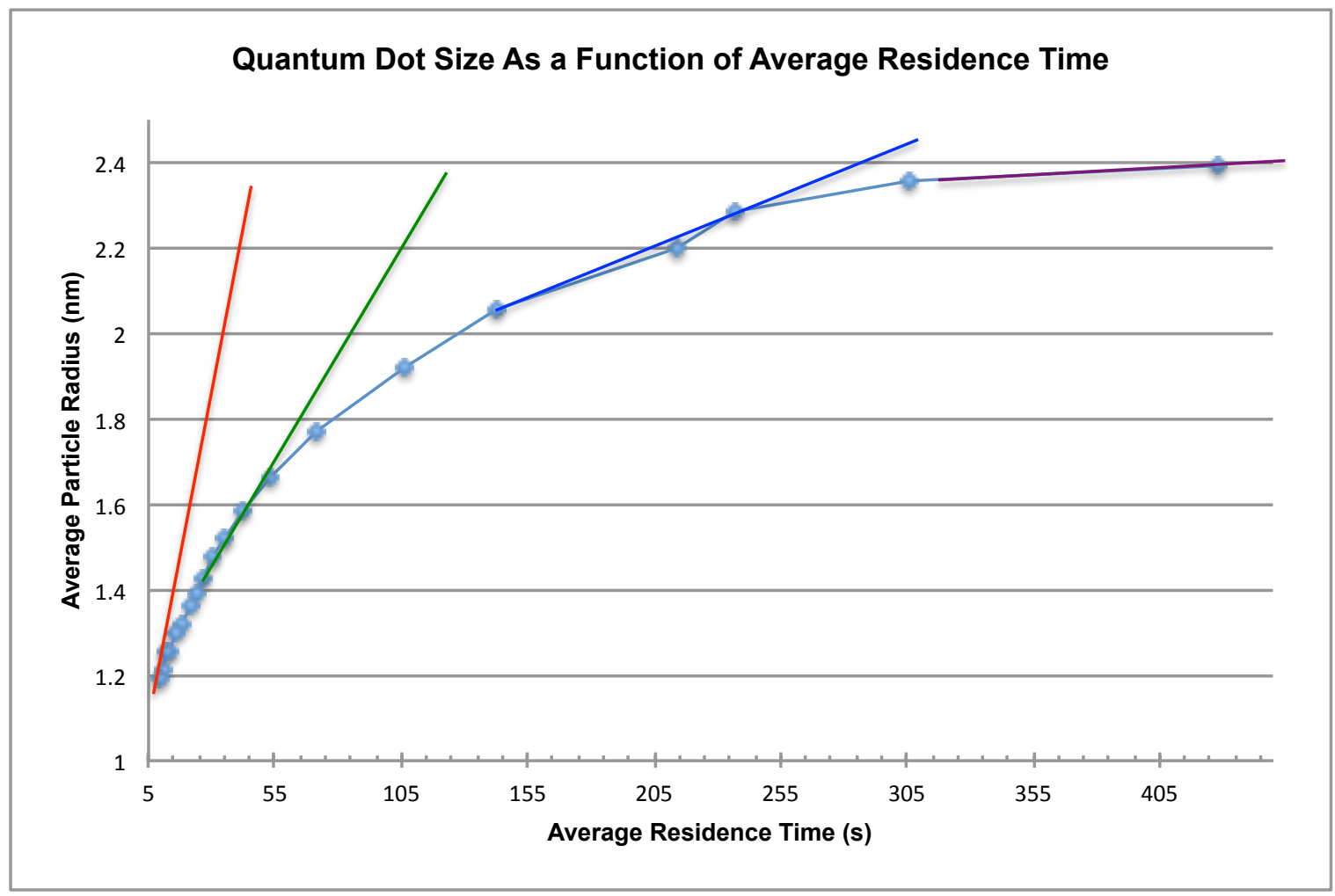

Figure 52: Average quantum dot radius as a function of residence time for all optimized reactor trials. The slope of red line represents the rate of particle growth at low residence times. The green line, blue line, and purple lines respectively represent decreasing growth rate with increasing residence time.

First residence time was calculated from volumetric flow rate using the reactor dimensions. Then the average center wavelength at each flow rate for all optimized reactor trials was converted to average quantum dot size using the University of Arkansas 
empirical equation relating particle size to center wavelength (Equation 9). The particle size vs. residence time relationship was then plotted using Microsoft Excel.

The plot on Figure 52 matches growth trends observed by Yen et al. (41) for CdSe quantum dot synthesis (Figure 53).
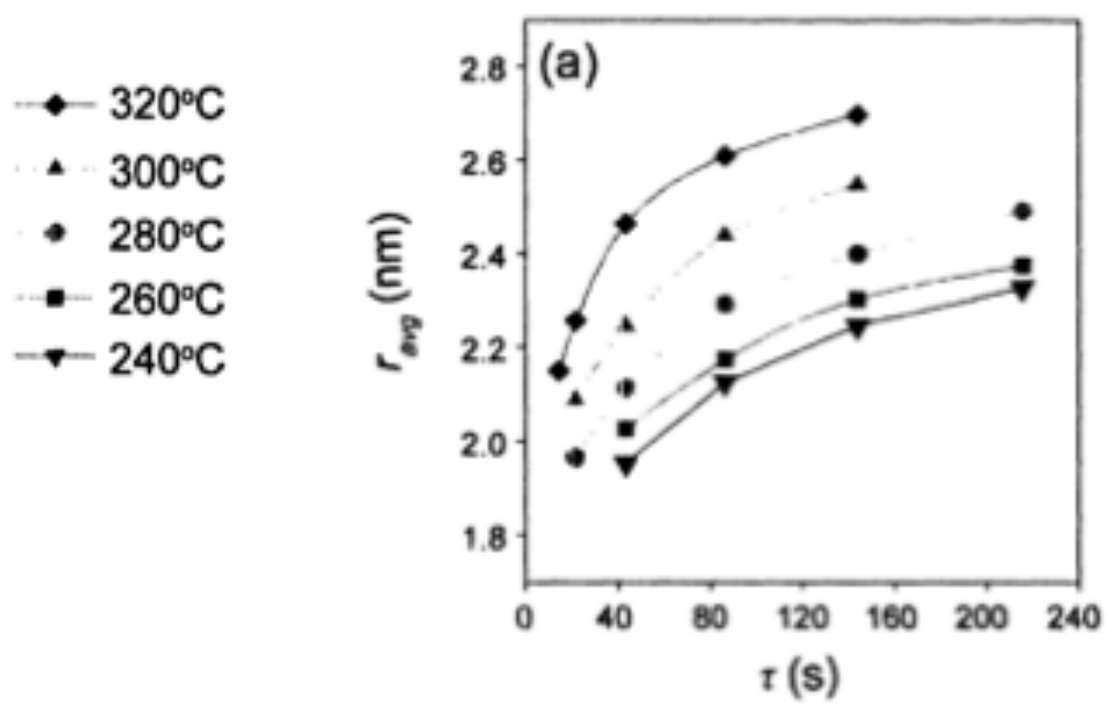

Figure 53: Quantum dot radius as a function of time in the heated section of the reactor. Note the curve at $240^{\circ} \mathrm{C}$ that matches the synthesis temperature used in this thesis. ${ }^{57}$

At short residence times quantum dot growth occurs rapidly as suggested by nucleation and growth theory outlined on Figure 7. Immediately upon reaching reaction temperature there is a nucleation burst and the newly formed nuclei grow rapidly due to a high degree of solution supersaturation. This phenomenon can be described by the red line on Figure 52 , the steep slope of the size vs. time profile shown by this red line is indicative of rapid growth. Growth occurs rapidly at this point because the reagent solution is supersaturated with a large quantity of reaction monomers. In diffusion-controlled reactions the mean distance the monomers must move through solution to be incorporated in the quantum dot nuclei is relatively short due the high concentrations. This results in a 
rapid growth rate. The reaction monomer concentration in solution decreases as the reaction progresses and the growth rate slows due to an increase in the mean diffusion distance. This phenomenon is demonstrated by the decreasing slope or "growth rate" of the green, blue, and purple lines respectively as time passes. As the monomer concentration in solution approaches the solubility limit of octadecene, the rate of incorporation of reaction monomers matches the rate of particle dissolution, and growth reaches equilibrium. Particles continue to grow to decrease surface energy in a phenomenon known as Ostwald ripening. In Ostwald ripening more stable nuclei, those larger than the critical radius, continue to grow as reaction monomers diffuse from less stable smaller nuclei. This in turn causes smaller nuclei to shrink decreasing the surface energy of the system. The near horizontal slope of the purple line could indicate that at residence times above 400 seconds growth occurs by Ostwald ripening and the concentration of reaction monomers is at the solubility limit of octadecene. 


\section{CONCLUSIONS}

A microfluidic Cd-Se quantum dot synthesis and analysis system was successfully fabricated, optimized, and characterized in this study. This system successfully produced Cd-Se quantum dots varying in fluorescence from 490-620nm at flow rates varying from $2 \mathrm{ml} / \mathrm{min}$ down to $0.05 \mathrm{ml} / \mathrm{min}$, respectively. Cd-Se quantum dots were confirmed to range in size from $2 \mathrm{~nm}$ to $10 \mathrm{~nm}$ using atomic force microscopy. Cd-Se precursors were shown to be capable of mixing at low temperature $\left(17^{\circ} \mathrm{C}\right)$ and treated as a single solution without the presence of significant nucleation and growth effects. Repeatability testing of fluorescence measurements was conducted for each flow rate and corresponding central wavelength. The average standard deviation of spectral peak center wavelength across all flow rates was within $6 \mathrm{~nm}$ for all reactors. The optimized 316 stainless steel reactor is the best characterized and appears to have the best process reliability, however, it also creates more polydisperse samples than the optimized TEFLON ${ }^{\circledR}$ reactor evidenced by the spectral tail. The optimized TEFLON ${ }^{\circledR}$ reactor with new octadecene produced the lowest standard deviation of all trials but the optimized 316 stainless steel reactor was not tested with new octadecene.

Residence time is the most important variable in this microfluidic synthesis method.

Ideally, the residence time should have been the same between the TEFLON ${ }^{\circledR}$ reactor and the stainless steel reactor; however, oxide growth in the stainless steel reactor reduced the stainless steel reactor's inner diameter. This both increased flow velocity through the reactor tubing and decreased residence time. This theory is supported by the fact that the TEFLON $^{\circledR}$ reactor created larger quantum dots than the stainless steel reactor at the same 
flow rates. Thermal transfer differences between the two tubing materials were determined to have a negligible effect on synthesis properties.

I recommend using the TEFLON ${ }^{\circledR}$ reactor for quantum dot synthesis unless a glass reactor is available.

Results indicate the largest source of polydispersity of synthesized quantum dots is residence time distribution inherent in single-phase flow. The most effective means to reduce polydispersity inherent in this reactor design is to redesign the reactor as a twophase flow reactor. This will be covered in greater detail in continuing research section. New chemicals should be obtained to ensure proper characterization and limit error caused by degradation and contamination from prior usage. New needles and septum should be used to limit oxygen contamination. The procedure outlined in the appendix should be followed to ensure proper operation of this microfluidic system.

Overall this microfluidic synthesis process has show greater control of nucleation and growth parameters than conventional bulk synthesis methodology previously employed at Cal Poly. Furthermore, real-time data acquisition with a fluorescence flow cell allowed for testing of a wide range of flowrates, limited sample consumption, detailed analysis of samples, and real time manipulation of reaction parameters to tune reaction characteristics.

\subsection{Continuing Research}

Several proposed design modifications were not investigated to limit the scope of this thesis. One such modification is the addition of a microfluidic mixer to mix the two precursor solutions prior to injection into the reactor. Room temperature nucleation was 
problematic to process reliability. The mitigation of room temperature nucleation and growth was accomplished using a chilling system that cooled the octadecene precursor mixture to just above its solidus temperature. Despite some limited success, the nucleation and growth reaction could not be fully inhibited. After four hours nucleation was shown to occur. This room temperature nucleation and growth keeps reaction conditions from remaining constant for the duration of a trial. Disparities resulting from the degree of nucleation before injection could be eliminated with the use of a microfluidic mixer. This mixer would allow precursor solutions to be kept separate until right before they are injected into the reactor. Additionally, a microfluidic T mixer separate from the reaction coil would keep time constant between mixing of the precursor solutions and injection into the reaction coil. PDMS could be used to fabricate the microfluidic-mixing channel as it has the required chemical stability in octadecene at low temperatures. Large input and output port slabs will be required to prevent blowout at the pressures experienced by this system.

For any subsequent work on this utilizing this microfluidic system it is important to note that thermal characteristics of the reaction bath vary with the position of the fume hoodsliding shield. When the shield is lowered, air is pulled at a higher velocity past the heating bath and the temperature was found to fluctuate up to $10^{\circ} \mathrm{C}$ based on hood position alone. A more accurate hotplate with a better feedback mechanism would keep temperature more constant, however, the current hotplate works well if the conditions within the fume hood are controlled.

Another modification is controlling the concentrations of reaction precursors. The relative amounts of precursors have been used to control the growth of Cd-Se quantum 
dots in literature. ${ }^{31,49}$ In trial 4_19_14 half the TOP-Se precursor solution was spilled and the center wavelength of quantum dots produced dropped significantly. This method potentially allows for production of larger quantum dots at constant temperature. In several instances coagulation of the precursor solutions was observed for several samples prior to injection into the reactor. A chilled ultrasonic water bath could be used following mechanical mixing to better disperse precursor solutions and minimize coagulation.

Increasing the length of tubing submerged in the oil bath will increase the residence time, possibly allowing for production of larger particles at the same flow rates. The use of glass capillary tubing would increase the rate of thermal transfer compared to the TEFLON $^{\circledR}$ reactor and would have a lower interior surface roughness than the 316 stainless steel reactor. This could decrease the spectral tail at high flowrates and increase monodispersity for all flow rates.

A multiphase flow microfluidic device would be ideal to implement with the presented synthesis method. Multiphase flow microfluidic devices create droplets of a second immiscible fluid (octadecene QD precursor solution) within fluid flowing in a microfluidic channel (Figure 54).

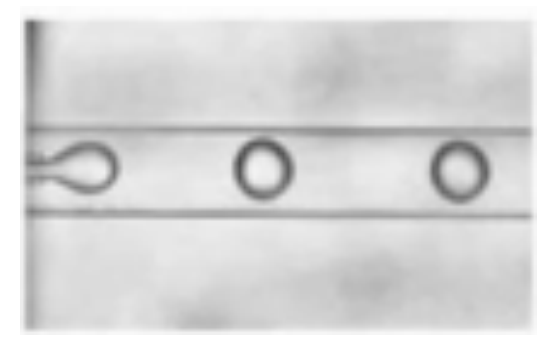

Figure 54: Multiphase flow microfluidic device. Note immiscible fluid drops within the channel. ${ }^{58}$ 
These droplets do not touch the channel walls and mixing occurs more rapidly within them than a solid laminar stream. This would eliminate the fluid shear effects and surface interactions like heterogeneous nucleation that contribute to nanocrystalline polydispersity. 


\section{REFERENCES}

$\dagger$ Much of the nucleation and growth theory section is adapted from:

B. K. H. Yen, A. Giinther, M. A. Schmidt, K. F. Jensen, M. G. Bawendi, Angew. Chem. Int. Ed. $2005,44,5447-5451$.

Dickerson, Bryan Douglas. Organometallic synthesis kinetics of CdSe quantum dots. Diss. Virginia Polytechnic Institute and State University, 2005.

Schaffer, James P. The Science and Design of Engineering Materials. 2nd ed. Boston: WCB McGraw-Hill, 1999. 292-305. Print.

1 Patel, Prachi. "Quantum Dots Are Behind New Displays." IEEE Spectrum, 13 June 2012. Web. 4 Apr. 2013.

2 Leutwyler, W. Klopper, S. Leutwyler Bürgi, and H. B. Burgl. "Semiconductor clusters, nanocrystals, and quantum dots." Science 271 (1996): 933-937. Print.

3 Gonsalves, Peter R. THE DESIGN AND FABRICATION OF A MICROFLUIDIC REACTOR FOR SYNTHESIS OF CADMIUM SELENIDE QUANTUM DOTS USING SILICON AND GLASS SUBSTRATES. Cal Poly Digital Commons. California Polytechnic State University - San Luis Obispo, Feb. 2012. Web. 31 May 2013.

4 Trivedi, Evan R., and Shelby L. Hatch. Synthesis and Size Dependent Properties of Cd-Se Quantum Dots. Department of Chemistry, Northwestern University, n.d. Web. 12 May 2013.

5 "Quantum Dot." Elec_Intro. N.p., 06 May 2010. Web. 15 May 2013. <http://www.elec-intro.com/EX/0515-17/quantum-dots.jpg>.

6 Schmid, G. Nanoparticles: From Theory to Application. New York: John Wiley \& Sons, Incorporated, 2004. Print.

7 R. Brown, Solid State Physics, An Introduction for Scientists and Engineers, Cal Poly Physics Dept, 2006.

8 Zumdahl, Steven S. Chemistry Principles. 4th ed. Boston: Houghton Mifflin, 2001. Print.

9 Arthur, Ekpekpo, and Akpojivi Lucky. "Synthesis and Characterization of Cds and Cd-Se Quantum Dots by UV-VIS Spectroscopy." Journal of Emerging Trends in Engineering and Applied Sciences (JETEAS) 4.2 (2013): 273-280.

10 Gonsalves, Peter R. THE DESIGN AND FABRICATION OF A MICROFLUIDIC REACTOR FOR SYNTHESIS OF CADMIUM SELENIDE QUANTUM DOTS USING SILICON AND GLASS SUBSTRATES. Cal Poly Digital Commons. California Polytechnic State University - San Luis Obispo, Feb. 2012. Web. 31 May 2013. 6. Print

11 Leutwyler, W. Klopper, S. Leutwyler Bürgi, and H. B. Burgl. "Semiconductor clusters, nanocrystals, and quantum dots." Science 271 (1996): 933. Print.

12 Gonsalves, Peter R. THE DESIGN AND FABRICATION OF A MICROFLUIDIC REACTOR FOR SYNTHESIS OF CADMIUM SELENIDE QUANTUM DOTS USING SILICON AND GLASS 
SUBSTRATES. Cal Poly Digital Commons. California Polytechnic State University - San Luis Obispo, Feb. 2012. Web. 31 May 2013. 8. Print

13 "Fluorescence Instrumentation." KU Chemistry Fluorescence. Kutztown University, 02 July 2011. Web. 02 Feb. 2013.

14 Schouten, J. C. "Microfluidic Reactors for Nanomaterial Synthesis." Advances in Chemical Engineering. Vol. 38. San Diego, CA: Academic, 2010. 195-228. Print.

15 van Dam, R. Michael (2006) Solvent-resistant elastomeric microfluidic devices and applications. Dissertation (Ph.D.), California Institute of Technology. http://resolver.caltech.edu/CaltechETD:etd$12052005-234258$

16 Kenis, Paul JA, Rustem F. Ismagilov, and George M. Whitesides. "Microfabrication inside capillaries using multiphase laminar flow patterning." Science 285.5424 (1999): 83-85.

17 "Micro Flow Cells." OceanOptics, n.d. Web. 13 Mar. 2013.

18 "FIAlab Applications." Flow Injection. FIAlab, n.d. Web. 11 Mar. 2013.

19 Trojanowicz, Marek. "Flow Injection Analysis." Introduction. University of Warsaw \& Institute of Nuclear Chemistry \& Technology, Warsaw. N.p.: World Scientific, n.d. Print.

20 "The Conventional Flow Injection Method." FIAlab Principle - Flow Injection. FIAlab, n.d. Web. 11 Mar. 2013.

21 Trojanowicz, Marek. "Flow Injection Analysis." Introduction. University of Warsaw \& Institute of Nuclear Chemistry \& Technology, Warsaw. N.p.: World Scientific, n.d. 1. Print

22 Salisbury, David F. "Quantum Dots That Produce White Light Could Be the Light Bulb's Successor." Vanderbilt News. Vanderbilt.edu, 20 Oct. 2005. Web. <http://news.vanderbilt.edu/2005/10/quantumdots-that-produce-white-light-could-be- the-light-bulbs-successor-59204/>.

23 Serap Günes, Niyazi Serdar Sariciftci, Hybrid solar cells, Inorganica Chimica Acta, Volume 361, Issue 3, 15 February 2008, Pages 581-588, ISSN 0020-1693, 10.1016/j.ica.2007.06.042.

24 Talbot, David. "TR10: Nanocharging Solar." Signallake.com. Technology Review, 12 Mar. 2007. Web. 29 Nov. 2011. <http://www.signallake.com/innovation/QuantumDotSolarCell031207.pdf>.

25 "New Instruments at UBuffalo Will Help Scientists Map Tumor Surfaces, Study Environmental Impact of Quantum Dots." NanoWerk. N.p., 10 Feb. 2011. Web. 08 Feb. 2013.

26 "Researchers Eye Risks of Quantum Dots." Nanitenews.com. Rice University, 03 Feb. 2009. Web. 05 Apr. 2013.

27 Yen, Brian KH. Microfluidic reactors for the synthesis of nanocrystals. Diss. Massachusetts Institute of Technology, 2007.

28 Agrawal, Anshul. "Chapter 22 Precipitation and Crystallization Kinetics." Scribd. Scribd, n.d. Web. 06 July 2014. <http://www.scribd.com/doc/94118306/Chapter-22-Precipitation-and-CrystallizationKinetics>. 3

29 Sattler, Klaus. "The energy gap of cluster nanoparticles, and quantum dots." Handbook of Thin Films Materials, Nanomaterials and Magnetic Thin Films 5 (2002): 61-97. 
30 Peng, X.; Wickham, J.; Alivisatos, A. P. J. Am. Chem. Soc. 1998, 120, 5343-5344.

31 Dickerson, Bryan Douglas. Organometallic synthesis kinetics of CdSe quantum dots. Diss. Virginia Polytechnic Institute and State University, 2005.

32 Schaffer, James P. The Science and Design of Engineering Materials. 2nd ed. Boston: WCB McGrawHill, 1999. 292-305. Print. 294

33 Mersmann, Alfons, ed. Crystallization technology handbook. CRC Press, 2001.

34 Askeland, Donald R., and Pradeep P. Fulay. The Science and Engineering of Materials. Southbank, Victoria, Australia: Thomson, 2006. Print.

35 Yen, Brian KH. Microfluidic reactors for the synthesis of nanocrystals. Diss. Massachusetts Institute of Technology, 2007. 46. Print.

36 "1-OCTADECENE." ChemicalBook---Chemical Search Engine. N.p., 2010. Web. 09 August. 2014.

37 “CES EduPack software, Granata Design Limited, Cambridge,UK,2009.”

38 "SU-8 2000: permanent epoxy negative photoresist," MicroChem, 26 Apr. 2012. [Online]. Available: www.microchem.com.

39 Gonsalves, Peter R. THE DESIGN AND FABRICATION OF A MICROFLUIDIC REACTOR FOR SYNTHESIS OF CADMIUM SELENIDE QUANTUM DOTS USING SILICON AND GLASS SUBSTRATES. Cal Poly Digital Commons. California Polytechnic State University - San Luis Obispo, Feb. 2012. (20-23)

40 "1-OCTADECENE." ChemicalBook---Chemical Search Engine. N.p., 2010. Web. 09 Jan. 2013.

41 "FIA Series Fluorescence Flow Cells - Ocean Optics." Ocean Optics. N.p., n.d. Web. 15 May 2013. $<$ http://oceanoptics.com/product/fia-series-fluorescence-flow-cells/>.

42 "Ultem ${ }^{\circledR}$." IDEX Health \& Science. IDEX Corporation, n.d. Web. 09 Jan. 2013.

43 Sparks, Laura C. DEVELOPMENT AND CHARACTERIZATION OF PHOSPHOLIPID ENCAPSULATED QUANTUM DOT CONSTRUCTS FOR BIOLOGIC APPLICATIONS. Cal Poly Digital Commons. California Polytechnic State University San Luis Obispo, June 2012. Web. 2 June 2013.

44 SpectraSuite. Program documentation. OceanOptics. Vers. 000-20000-300-02- 201110. Halma Group Company, n.d. Web.

45 Dabbousi, B. O., et al. "(Cd-Se) ZnS core-shell quantum dots: synthesis and characterization of a size series of highly luminescent nanocrystallites." The Journal of Physical Chemistry B 101.46 (1997): 9463-9475.

46 Lichtner, Aaron. Process Development and Characterization of Cadmium Selenide Quantum Dots Synthesis through Nanoparticle Size Optimization. California Polytechnic State University, 5 Jan. 2009. Print.

47 Schaffer, James P. The Science and Design of Engineering Materials. 2nd ed. Boston: WCB McGrawHill, 1999. 292-305. Print. 
48 Kalloniatis, Michael, and Charles Luu. "Color Perception." 2011-03-30)[2012-09-01]. http://webvision. meal. utah. edu/book/part-viii-gabac-receptors/color-perception (2011).

49 Choi, Sang-Hyun, et al. "Synthesis of size-controlled Cd-Se quantum dots and characterization of CdSe-conjugated polymer blends for hybrid solar cells." Journal of Photochemistry and Photobiology A: Chemistry 179.1 (2006): 135-141.

50 Dickerson, Bryan Douglas. Organometallic synthesis kinetics of CdSe quantum dots. Diss. Virginia Polytechnic Institute and State University, 2005. 43

51 Agrawal, Anshul. "Chapter 22 Precipitation and Crystallization Kinetics." Scribd. Scribd, n.d. Web. 06 July 2014. <http://www.scribd.com/doc/94118306/Chapter-22-Precipitation-and-CrystallizationKinetics>. 10

52 Yen, Brian KH. Microfluidic reactors for the synthesis of nanocrystals. Diss. Massachusetts Institute of Technology, 2007. 25. Print.

53 De Yoreo, James J., and Peter G. Vekilov. "Principles of crystal nucleation and growth." Reviews in mineralogy and geochemistry 54.1 (2003): 64.

54 De Yoreo, James J., and Peter G. Vekilov. "Principles of crystal nucleation and growth." Reviews in mineralogy and geochemistry 54.1 (2003): 65.

55 "Properties of Molecule: 1-Octadecene." Properties of Molecule: 1-Octadecene. 2003-2013 Wiley Information Services, n.d. Web. 25 July 2014.

$<$ http://www.infotherm.com/static/molpages/02/65/mol26572.html>.

56 Chan, Emory M.; Alivisatos, A. Paul; \& Mathies, Richard A.(2005). High-Temperature Microfluidic Synthesis of CdSe Nanocrystals in Nanoliter Droplets. Lawrence Berkeley National Laboratory: Lawrence Berkeley National Laboratory. Retrieved from: http://www.escholarship.org/uc/item/6cd301zm

57 Yen, Brian KH. Microfluidic reactors for the synthesis of nanocrystals. Diss. Massachusetts Institute of Technology, 2007. 41. Print.

58 Cunther, Axel, and Michiel T. Kreutzer. "Multiphase Flow." Micro Process Engineering: A Comprehensive Handbook 1 (2009). 


\section{APPENDICES}

\section{Appendix A Microfluidic Quantum Dot Synthesis Required Supplies}

\section{Chemicals:}

65 mg Cadmium Oxide Powder

33 mg Selenium Powder

$3 \mathrm{ml}$ Oleic Acid (tech grade 90\%)

$60 \mathrm{ml}$ Octadecene (tech grade $90 \%$ )

$0.4 \mathrm{ml}$ Trioctylphosphine (tech grade $90 \%$ )

Acetone for cleanup

\section{Equipment:}

1 - 50ml 14/20 2-neck Round Bottom Flask

1 - Small Stir Bar (For Selenium Precursor)

1 - 100ml 14/20 3-neck Round Bottom Flask

1 - Medium Stir Bar (For Cadmium Precursor)

$6-14 / 20$ Rubber Septa

1 - Large Rubber Septa

1-1000ml Pyrex Beaker

1 - 10cc Luer Lock Tip Syringe (Octadecene)

1- 1ml Disposable Plastic Syringe (Trioctylphosphine)

1 - 3ml Disposable Plastic Syringe (Oleic Acid)

2 - Veterinary Tip, 18 gauge, 3-inch SS Needle 
1 - 50cc Micro-Mate Luer Lock Tip Glass Syringe

1 - Large Stir Bar (Chilled Water Bath)

1 - Glass Thermometer

Borosilicate Vials of Desired Size (sample collection)

Hot/Stir Plate with RTD Probe Crystallization Dish

400ml - High Temperature Silicone Oil

$1-100$ X 500 Pyrex ${ }^{\circledR}$ Dish

$1-50 \mathrm{ml}$ Pyrex ${ }^{\circledR}$ Erlenmeyer Flask

1- Barnstead Thermolyne CIMAREC ${ }^{\circledR}$ Hotplate

1- EXTECH Instruments Thermocouple

8' Zeus PTFE ID: 1/32-inch OD: 1/16-inch Tubing for reactor and plumbing

2’ Zeus PTFE ID: 1/16-inch Tubing for coupling tubing

30-inch 316 Stainless Steel Tubing 1/32-inch OD: 1/16-inch for reactor

New Era NE-300 Syringe Pump

Thor Labs ${ }^{\circledR}$ LED-UV $385 \mathrm{~nm}$

Ocean Optics ${ }^{\circledR}$ USB4000 Spectrometer

2- Ocean Optics ${ }^{\circledR}$ Fiber Optic Cable

Ocean Optics ${ }^{\circledR}$ SpectraSuite ${ }^{\circledR}$ operating software

Ocean Optics ${ }^{\circledR}$ SMA Flow Cell

Kimwipes

Nitrile Gloves

Safety glasses 


\section{Appendix B Quantum Dot Standard Operating Procedure (SOP)}

\section{First Steps}

1) Turn on the UV LED light source by turning the power controller nob (a below) all the way to full. Then allow the LED to come to equilibrium (b below).

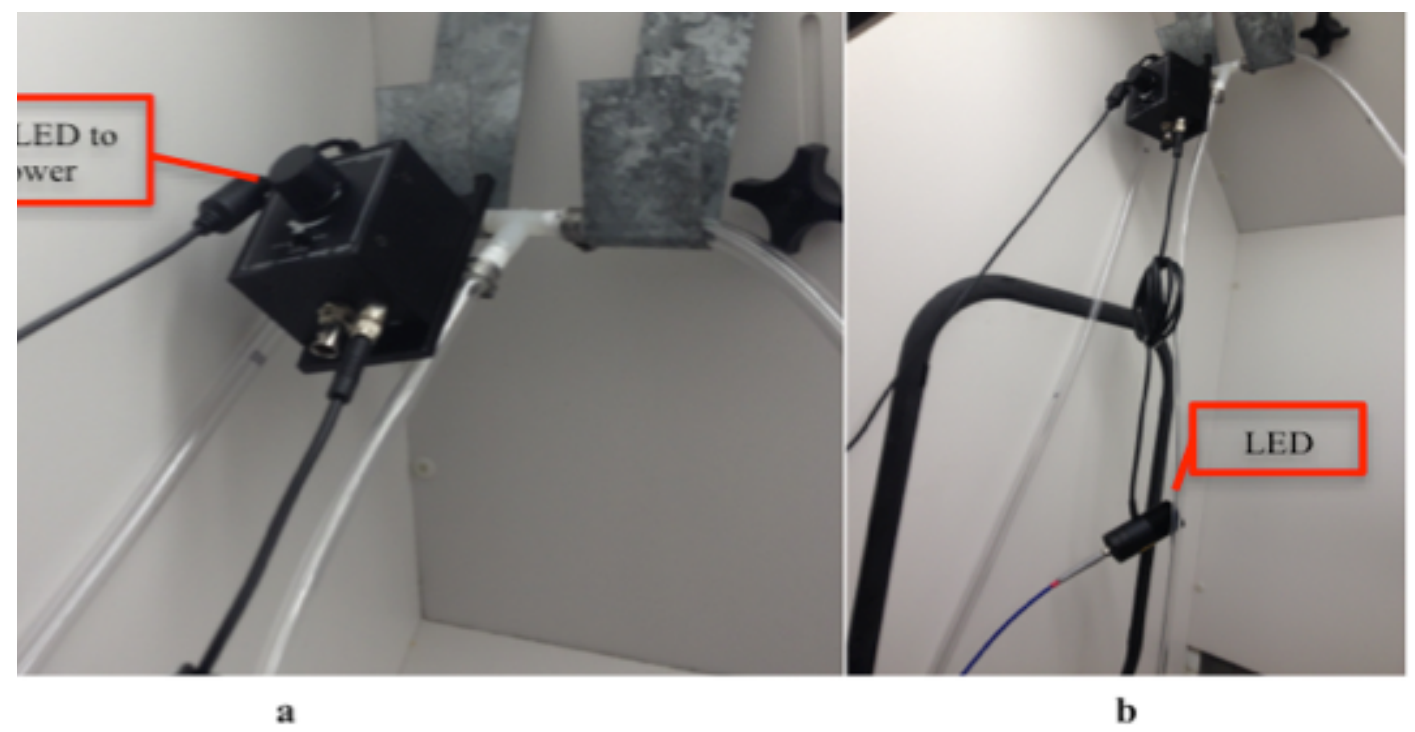

2) Make sure vent line for nitrogen system (below) feeds into a beaker filled with water to prevent oxygen backfill.

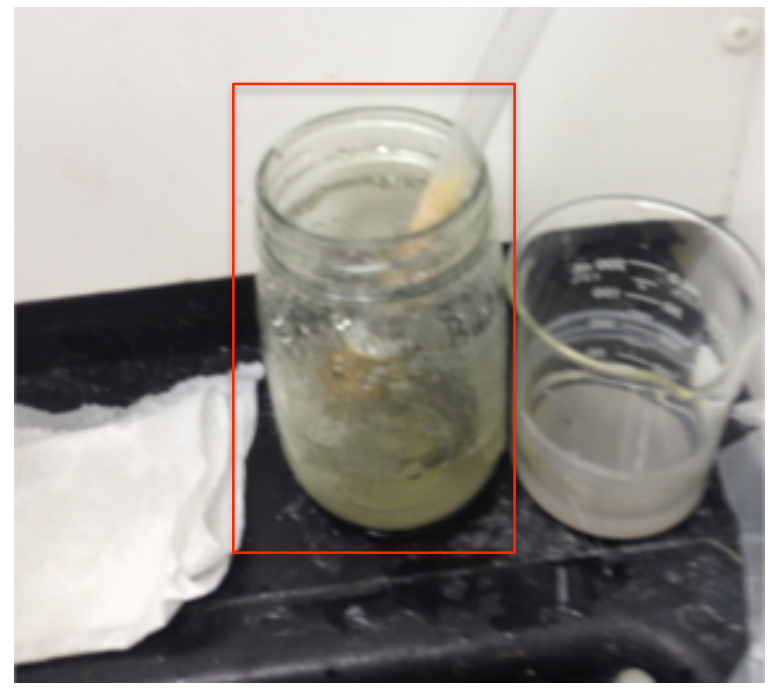




\section{Syringing process}

A process to transfer reactant chemicals without inducing oxygen contamination was required to ensure repeatability of reaction conditions. The procedure is outlined in the list below.

1) Insert nitrogen line needle and vent needle into rubber septum and flush out oxygen in both the original chemical container and receptacle for 10 minutes (a below), (b below).

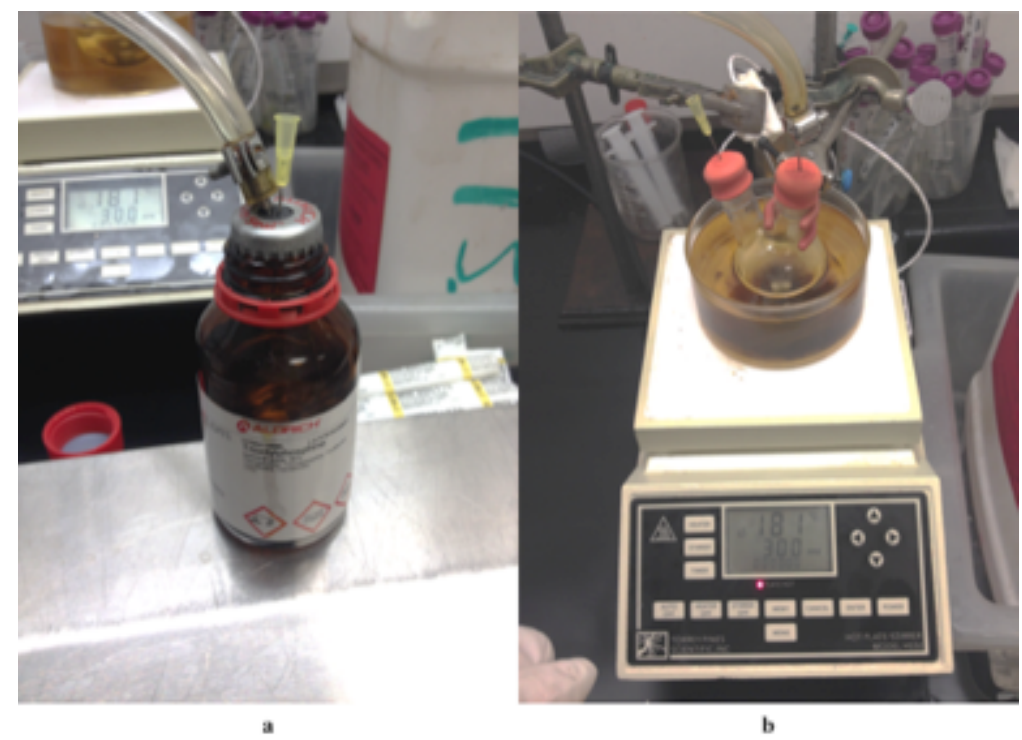

2) Obtain syringe of desired volume and attach needle to the leur lock tip (below).

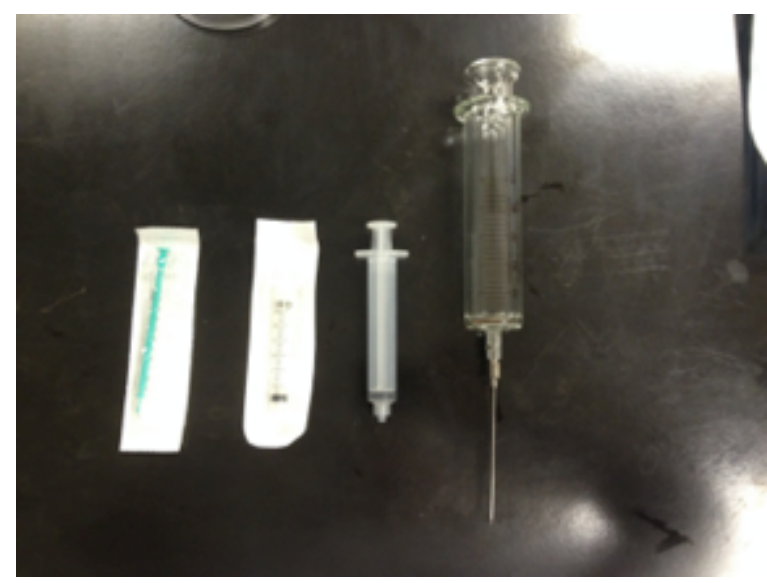


3) If using a glass syringe the syringe piston must be thoroughly lubricated with octadecene to prevent oxygen from slipping through the glass on glass gap.

4) Remove vent needle from the initial container to create positive pressure.

5) Insert the syringe needle through the rubber septum into the nitrogen on the other side.

6) Draw nitrogen from the container into the syringe and expel it into atmosphere three times to purge any oxygen trapped inside.

7) Draw the chemical/solution out of the initial container and transfer it to the purged receptacle container.

\section{Precursor Synthesis Procedure}

1. Obtain one $100 \mathrm{ml}$ three-neck flask and one two-neck $50 \mathrm{ml}$ flask

2. Thoroughly clean both flasks with acetone and dry them

3. Add a stir bar to each flask.

4. Measure $33 \mathrm{mg}$ selenium powder using a measuring stick (a below) into a scoopula resting on a scale (b below). Make sure to transfer powder close to the tip of the scoopula to ensure easy transfer to flasks ( $d$ below). 


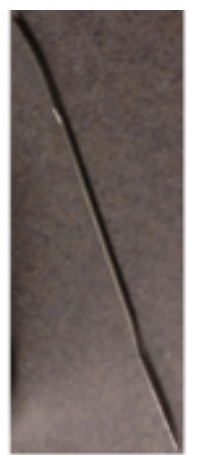

a

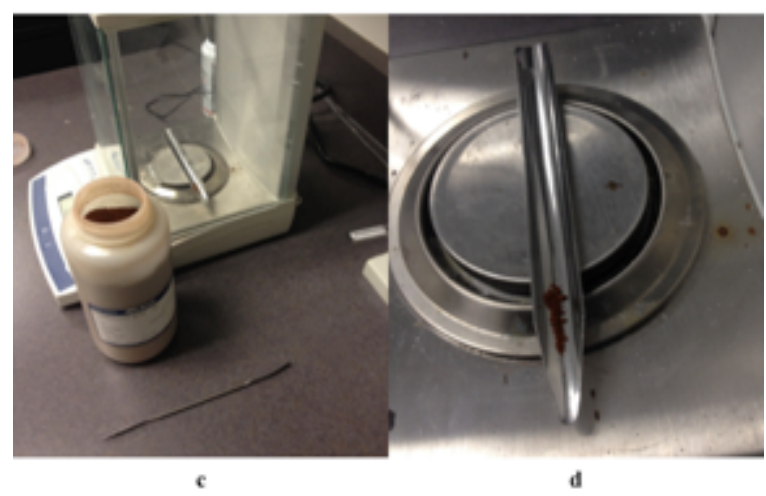

5. Transfer the powder into the $50 \mathrm{ml}$ two-neck flask and cap both necks with rubber septum.

6. Measure $65 \mathrm{mg}$ cadmium oxide using the same process (c above), (d above) and transfer it to the $100 \mathrm{ml}$ three-neck flask.

7. Keep the flasks upright using small dishes to ensure all powder precursors remains at the bottom of the flask. This ensures a complete reaction.

8. Suspend the selenium precursor in the $50 \mathrm{ml}$ two-neck flask over a high temperature silicon oil reaction bath heated to $150^{\circ} \mathrm{C}$ with a stir speed of $500 \mathrm{rpm}$.

9. Purge the flask of oxygen using a nitrogen needle and vent needle for 10 minutes.

10. Transfer the octadecene (a below) to container (b below) and purge for $10 \mathrm{~min}$. 

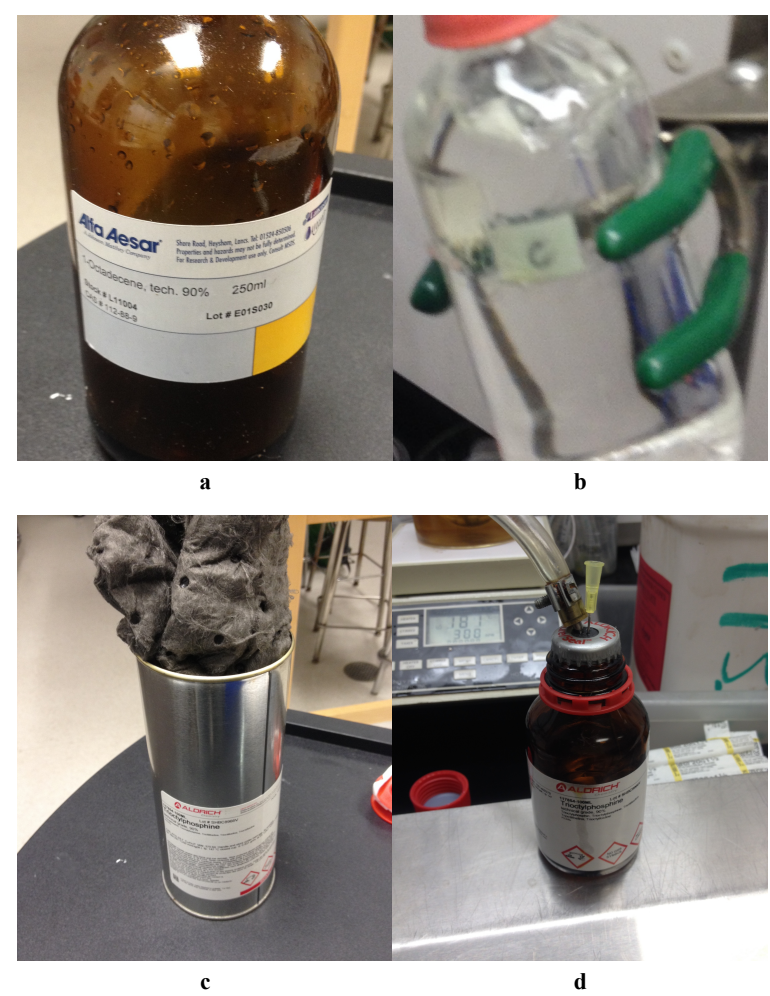

11. Remove trioctylphosphine from the protective cylinder (c above)

12. Purge trioctylphosphine ( $\mathrm{d}$ above) for $10 \mathrm{~min}$

13. Draw $10 \mathrm{ml}$ octadecene using a $10 \mathrm{ml}$ syringe using the syringing process.

14. Transfer $5 \mathrm{ml}$ of the $10 \mathrm{ml}$ octadecene drawn into the syringe into the selenium twoneck reaction flask.

15. The remaining $5 \mathrm{ml}$ will be used later to lubricate the $50 \mathrm{ml}$ glass syringe and to purge the microfluidic line of oxygen before synthesis.

16. Suspend the selenium precursor flask in the high temperature oil bath at $150^{\circ} \mathrm{C}$.

17. Once the octadecene has come to reaction temperature (about $5 \mathrm{~min}$ ) add $0.4 \mathrm{ml}$ trioctylphosphine (a below) to the selenium two-neck reaction flask (b below) using the syringing process. 


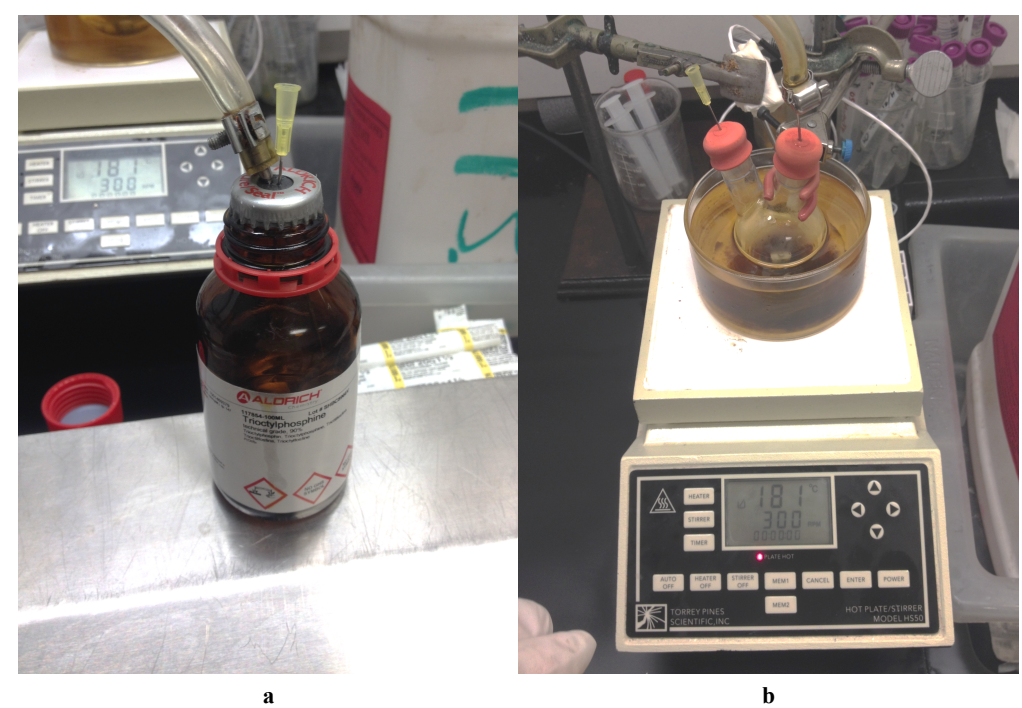

18. Continually purge the selenium two-neck reaction flask with nitrogen for the duration of the synthesis process ( $b$ above).

19. Leave this solution for 10-20 minutes until the black selenium powder is fully dissolved.

20. Remove selenium precursor from the heat, clean the bottom of the flask with paper towel, and place upright in a small dish to cool. Make sure to remove the vent needle but leave the nitrogen needle in to prevent any oxygen from contaminating the solution. Cadmium (a below) and selenium (b below) precursors shown below.

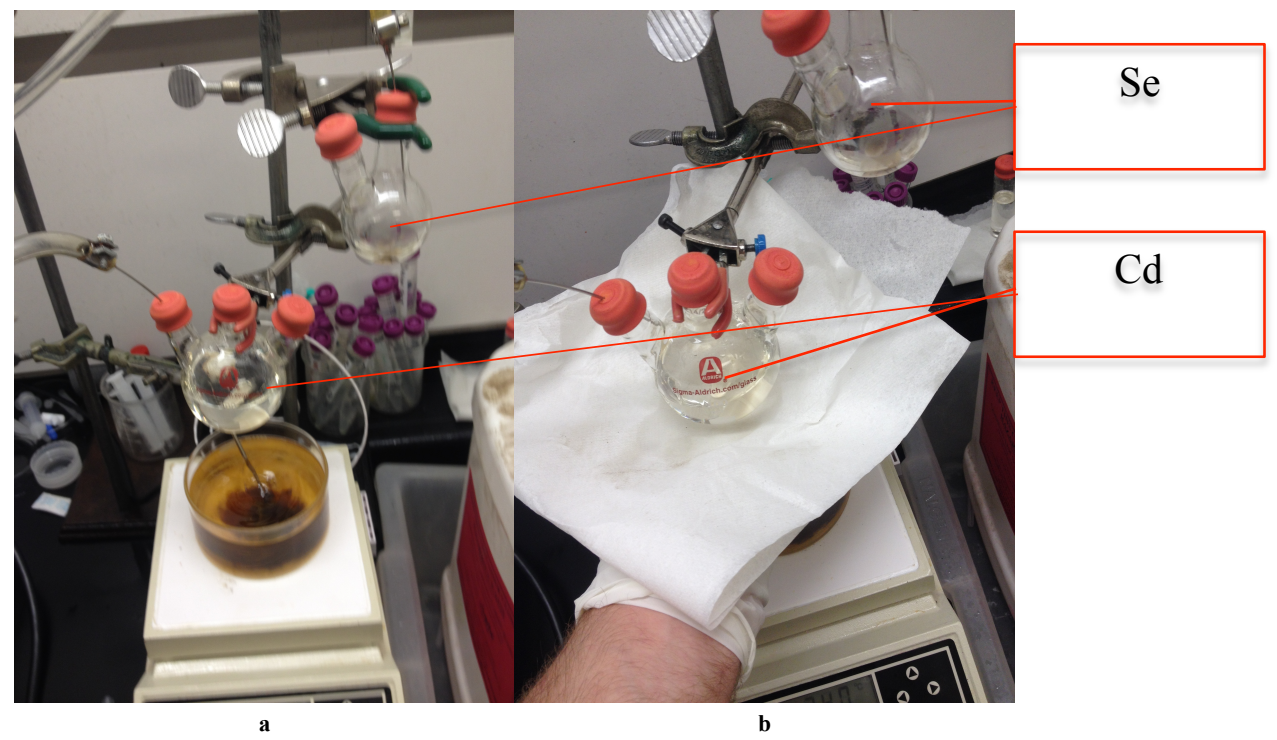




\section{Cadmium Precursor Synthesis}

1. Raise the high temperature silicon oil bath to $265^{\circ} \mathrm{C}$ with a stir speed of 500rpm.

2. Suspend the cadmium oxide three-neck reaction flask in the reaction bath and purge for 10 min using the same process as the selenium precursor solution.

3. Once purged transfer $50 \mathrm{ml}$ of purged octadecene into the cadmium oxide three-neck reaction flask using the syringing process.

4. After the reaction flask reaches temperature (about $15 \mathrm{~min}$ ), add $3 \mathrm{ml}$ of purged oleic acid using the syringing process.

5. Purge the flask for the duration of the reaction to remove oxygen as the cadmium oxide reacts.

6. After the addition of the oleic acid the cadmium precursor solution changes color from a dull red to clear (about 30 minutes).

7. Remove the cadmium from the silicon oil bath, clean the bottom of the flask with paper towel, and place upright in a small dish to cool (a below).

8. Remove the vent needle for the duration of cooling to create positive pressure in the cadmium precursor flask (b below). 


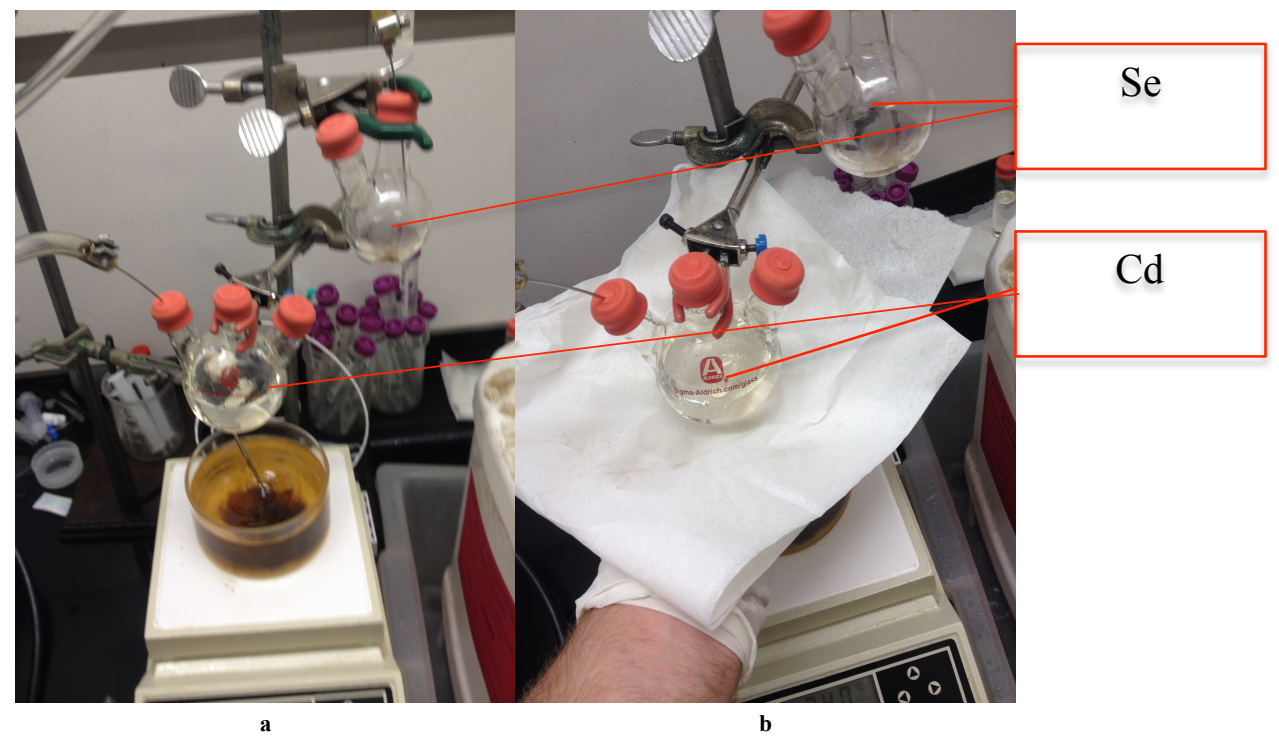

9. Remove cadmium precursor from the heat, clean the bottom of the flask with paper towel, and place upright in a small dish to cool. Make sure to remove the vent needle but leave the nitrogen needle in. This prevents any oxygen contamination (a above) and selenium ( $b$ above). 


\section{Precursor Solution Mixing Procedure}

1. Fill a large beaker with chilled water at $14^{\circ} \mathrm{C}$ and add a stir bar for mixing.

2. Place the beaker on a cool hotplate (a below) with the stir function enabled at $500 \mathrm{rpm}$.

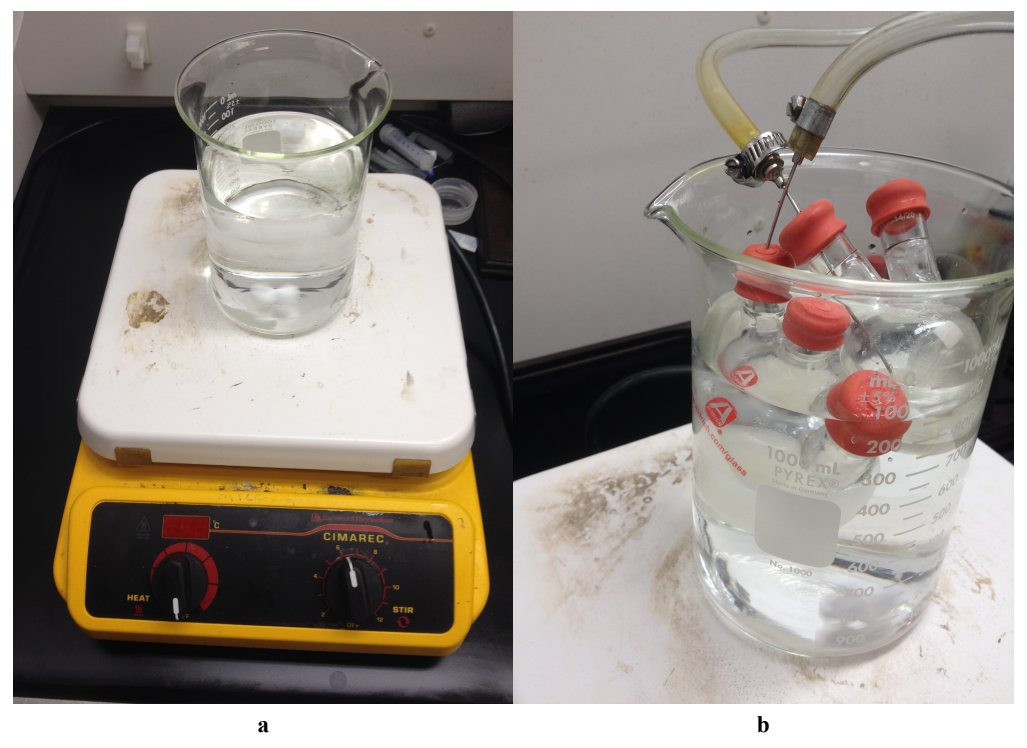

3. Place both precursor flasks in the chilled water bath and adjust them so the cadmium precursor stir bar mixes the cadmium precursor solution. The selenium precursor does not require mixing ( $b$ above). 
4. While waiting for the precursors to chill prepare a $50 \mathrm{ml}$ syringe by attaching a twoway leur lock valve and a veterinary needle in line with the syringe (a).
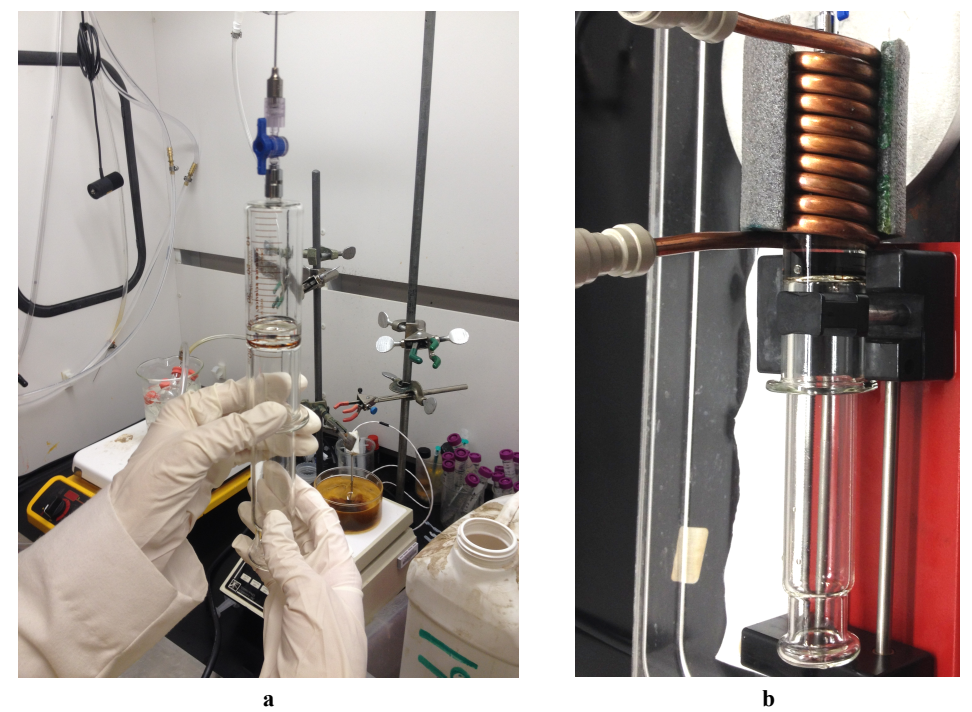

5. Lubricate the syringe piston with a small amount of octadecene left over in the $10 \mathrm{ml}$ syringe.

6. Chill the syringe using the copper coil chiller attachment.

7. When the solution becomes sufficiently chilled the cadmium precursor solution becomes slightly slushy and needs to be removed from the chilled water bath (a).

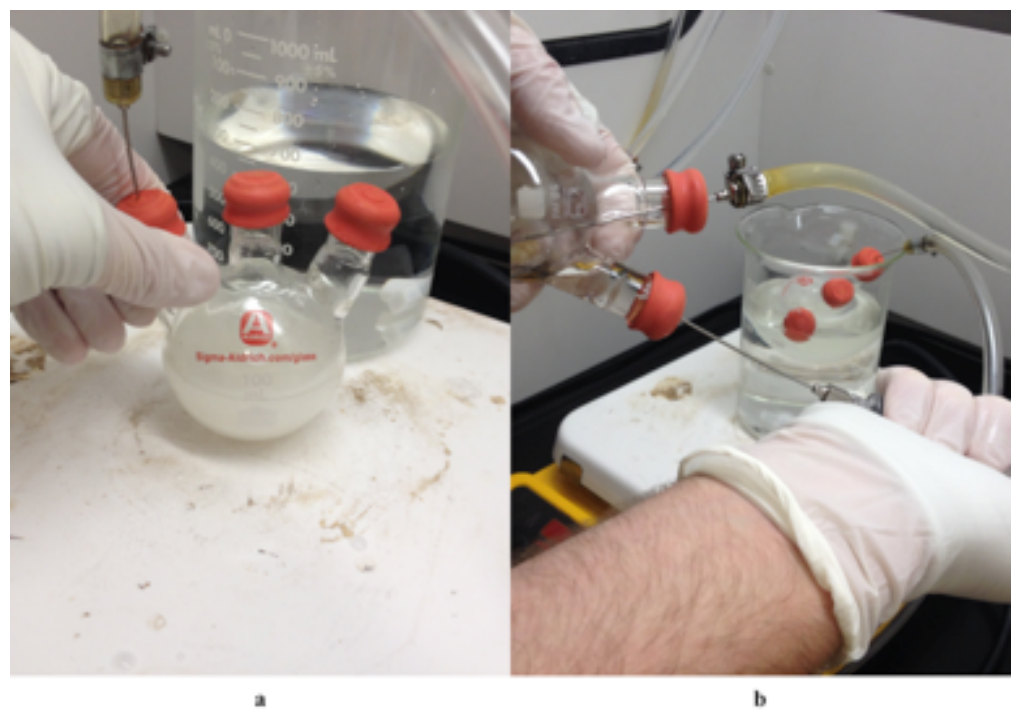


8. Add the selenium precursor solution to the cadmium precursor solution using the syringing process ( $\mathrm{b}$ above).

9. Mix the combined precursor solutions for two minutes by holding the 3-neck flask above the stir enabled hot plate ( $b$ above).

10. Remove the chilled 50ml syringe from the copper-chilling coil (a below) and transfer the chilled solution using the syringing process to the chilled $50 \mathrm{ml}$ glass syringe $(\mathrm{b}$ below).

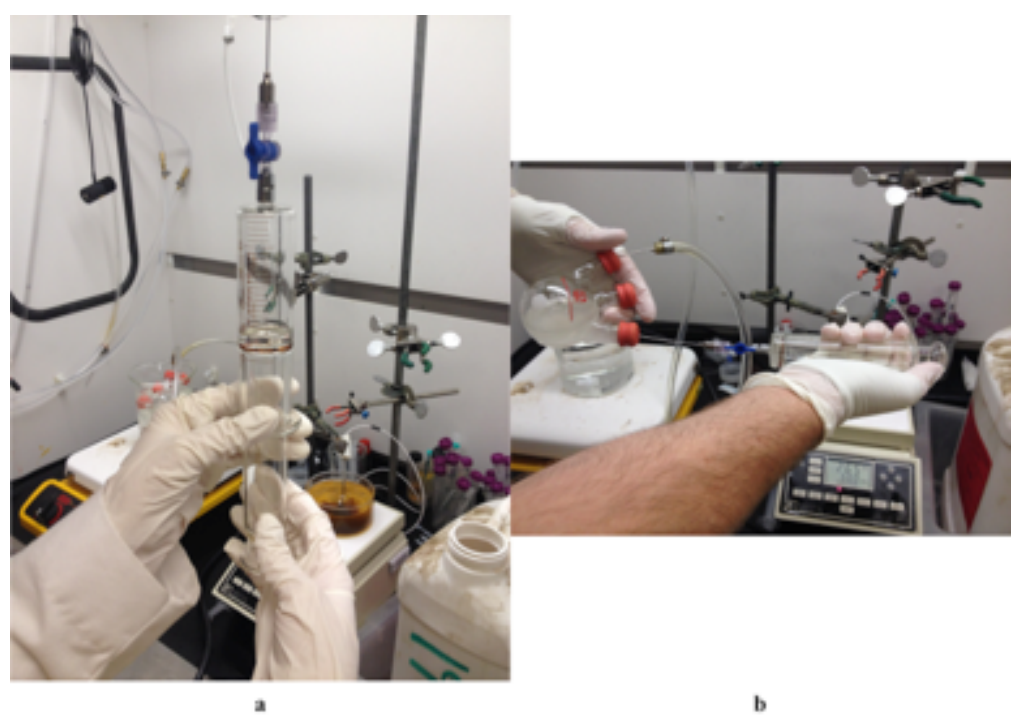


11. Replace the copper coil and insulation (below)

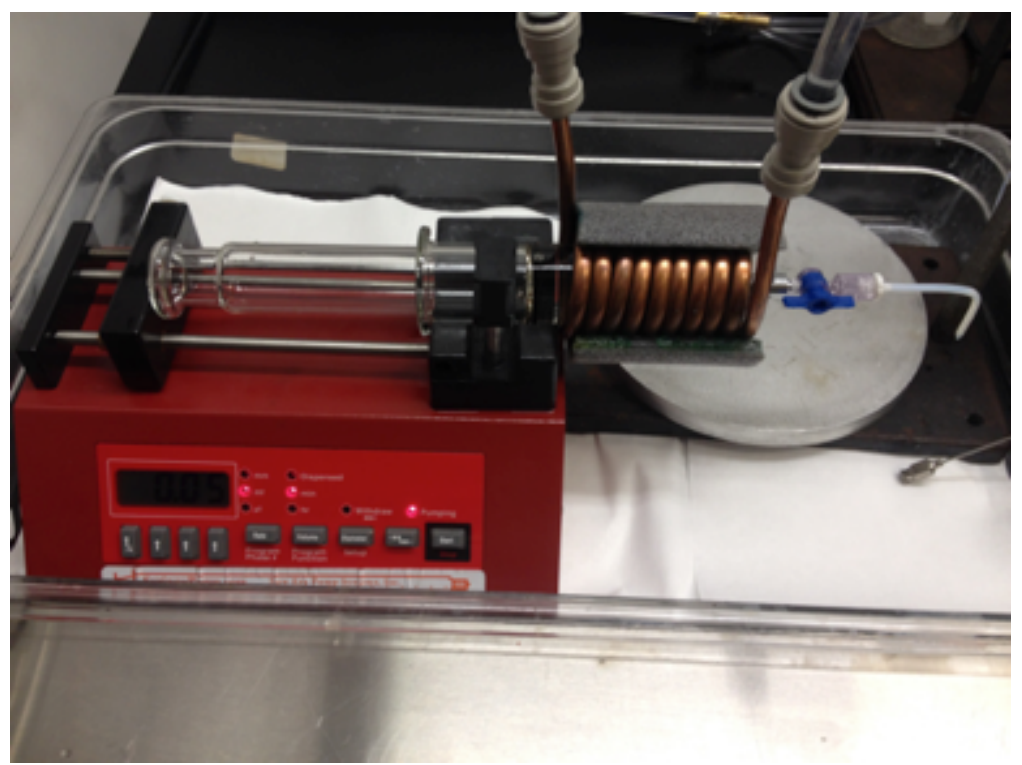

12. Load the $50 \mathrm{ml}$ syringe into the syringe pump (above).

13. Dispense the remaining octadecene in the $10 \mathrm{ml}$ syringe (used earlier to lubricate the $50 \mathrm{ml}$ syringe) into the reaction coil using hand pressure. This will purge oxygen from the tubing (a).

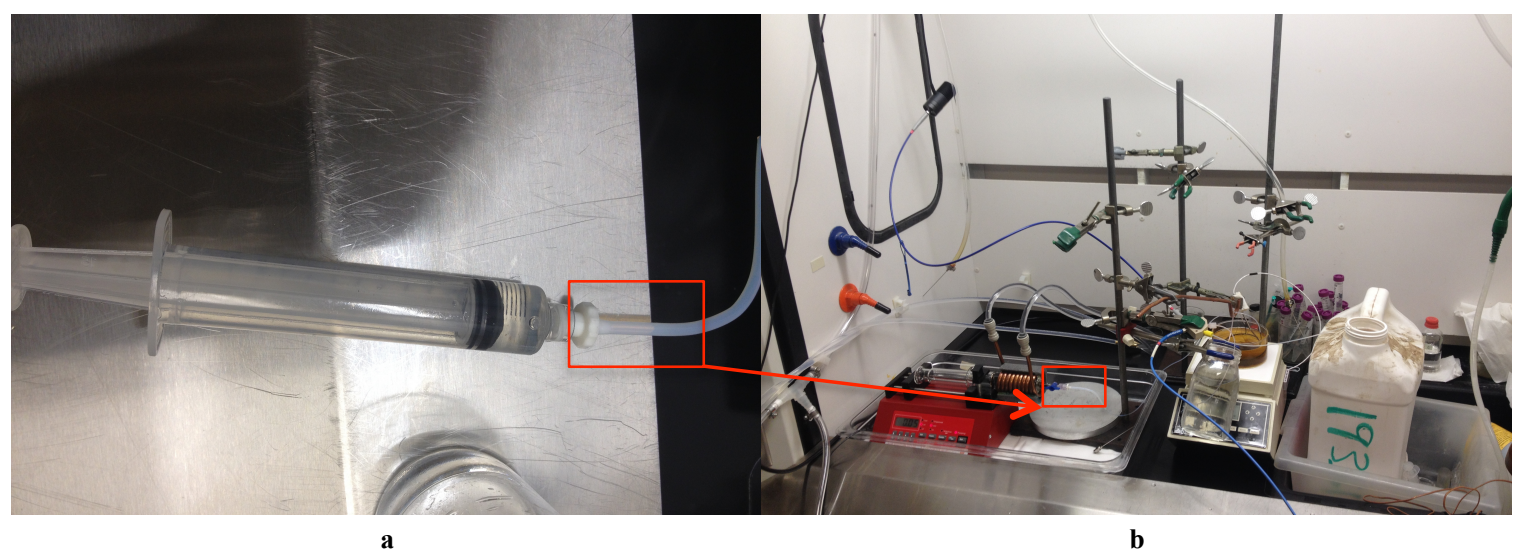

14. Attach the $50 \mathrm{ml}$ syringe to the reactor coil following purging (b above).

15. Add room temperature water to the chilling bath.

16. Test the flow cell and SpectraSuite by obtaining readings from the spectrometer. 
17. Establish a flow rate of $1 \mathrm{ml} / \mathrm{min}$ using the syringe pump to and dispense for $2 \mathrm{~min}$ to completely flush the reactor of pure octadecene.

18. Attach all fiber optic cables to their respective ports on the flow cell and attach the USB fiber optic spectrometer to the computer (below).

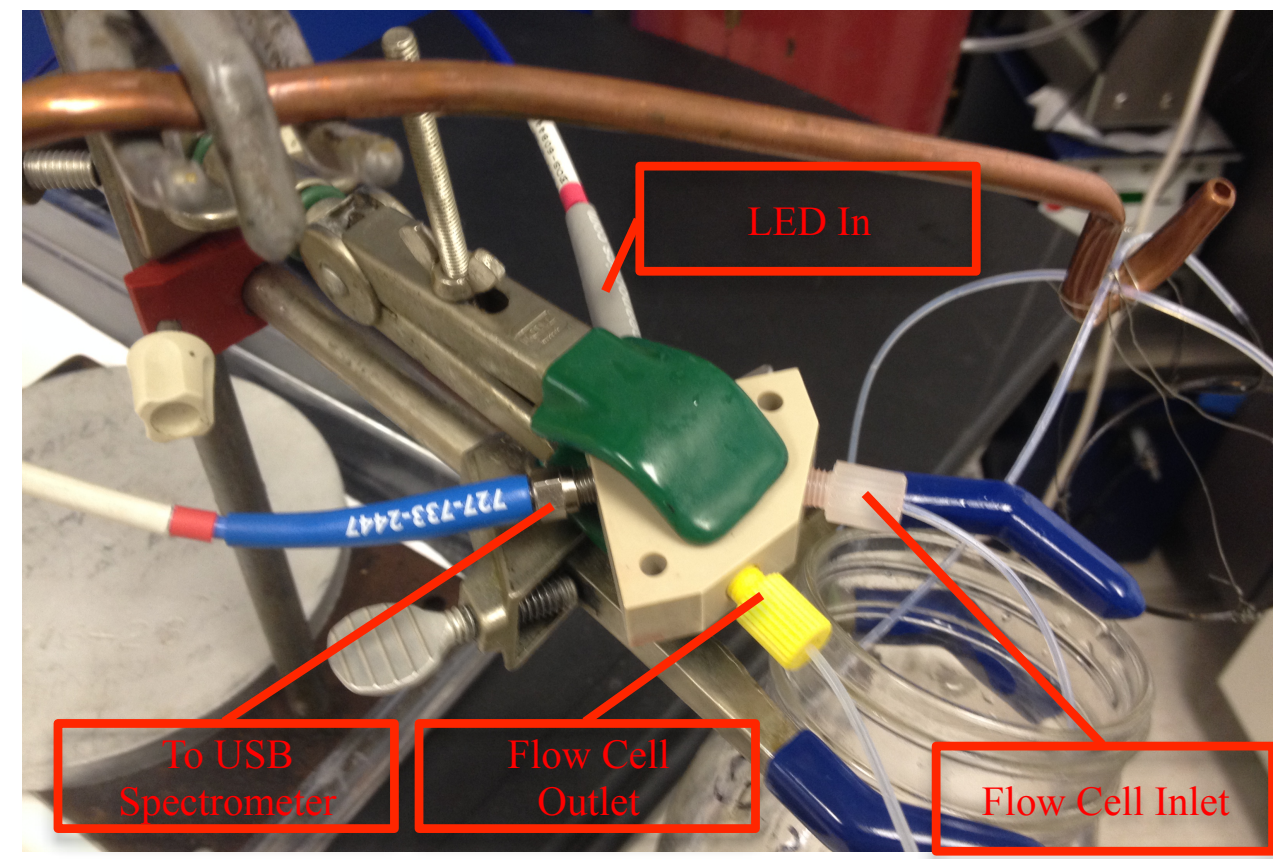

19. Take light spectrum measurement with SpectraSuite

20. Briefly turn off the UV LED and take a dark spectrum measurement with SpectraSuite

21. Start synthesis of quantum dots. 


\section{Appendix C MATLAB Code}

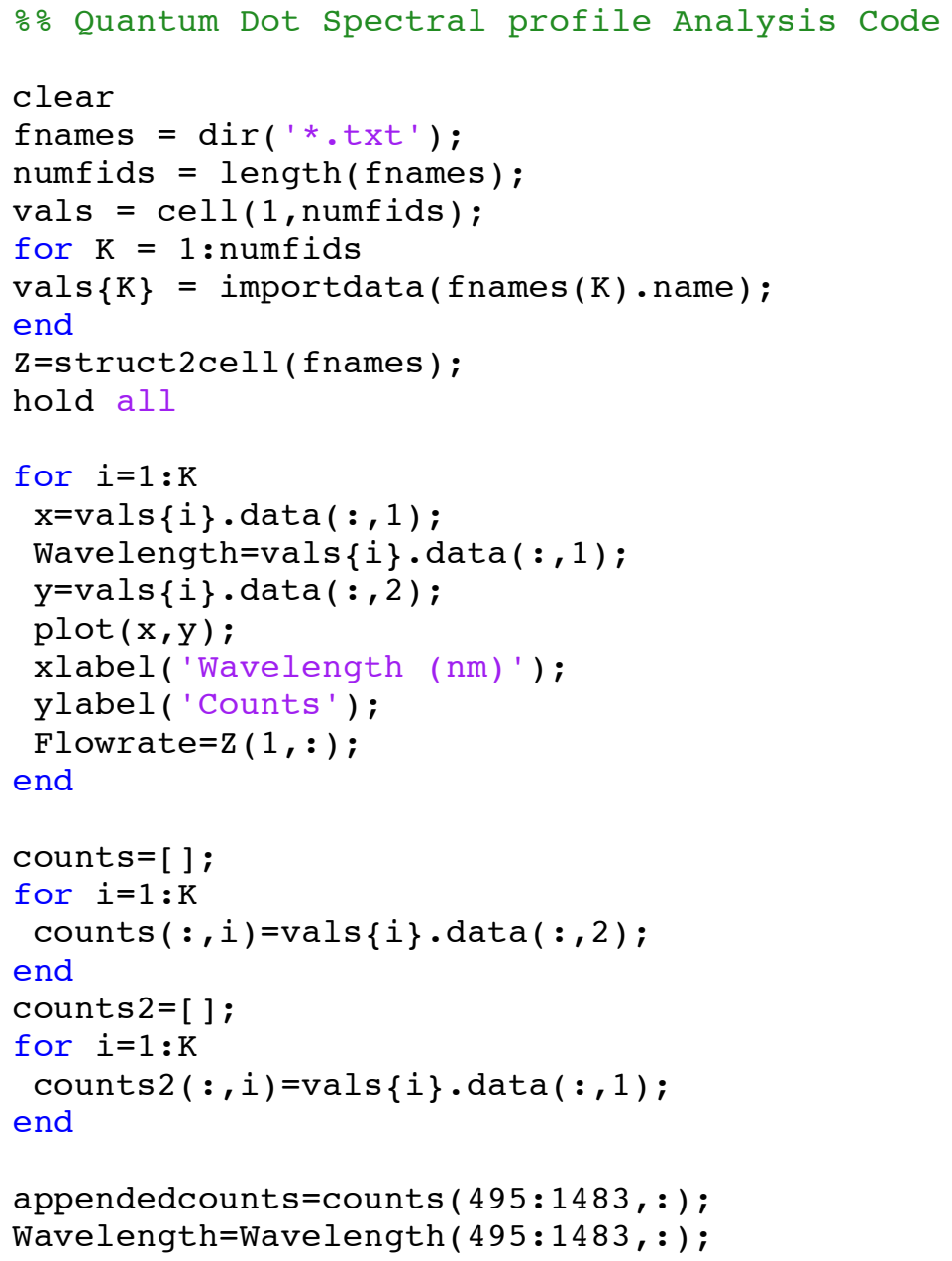




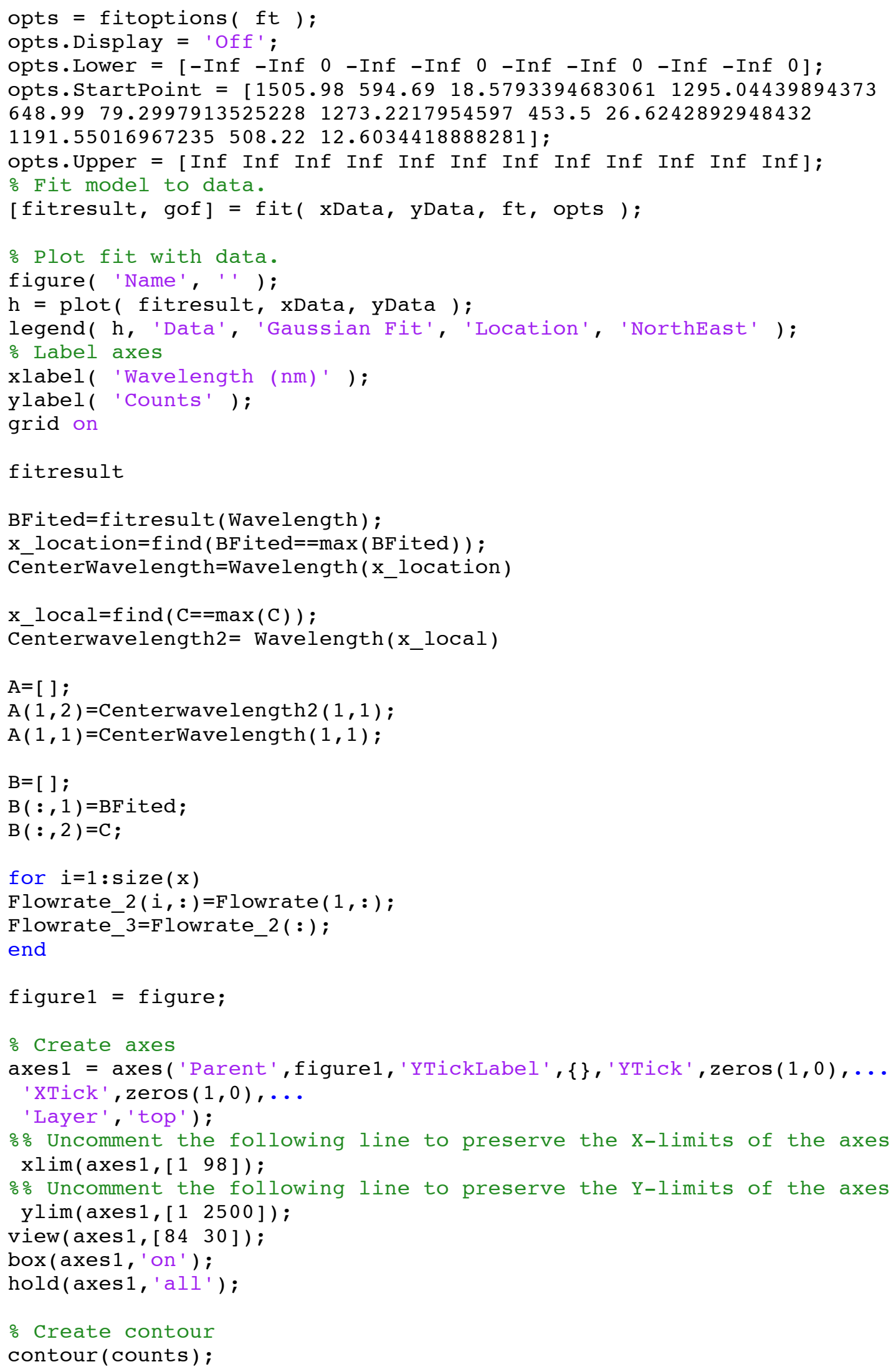




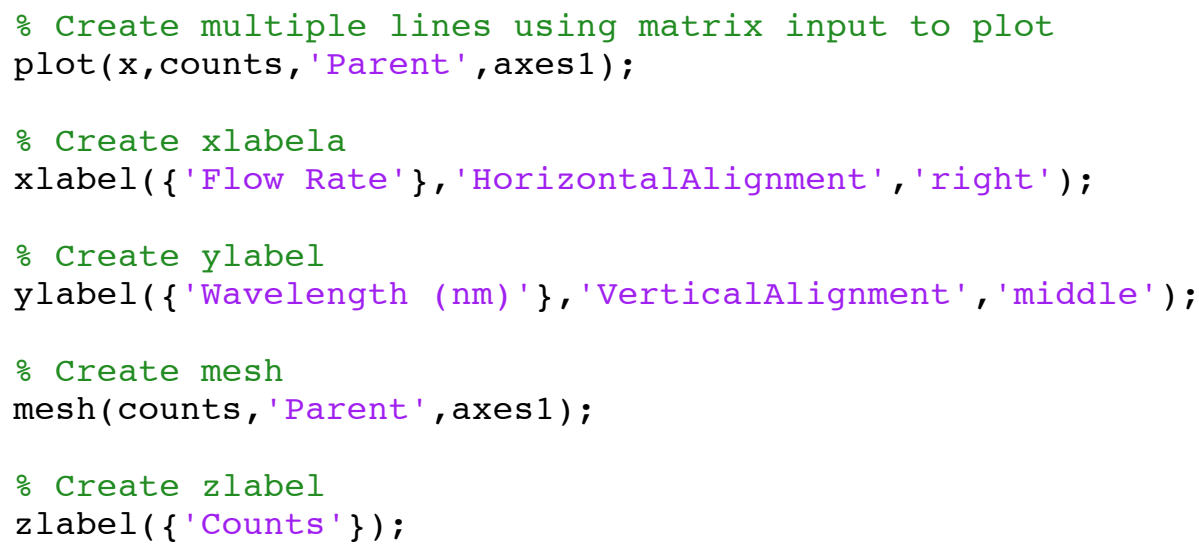


Appendix D 316 Stainless Steel Reactor Two-Dimensional Spectral Profiles By Trial

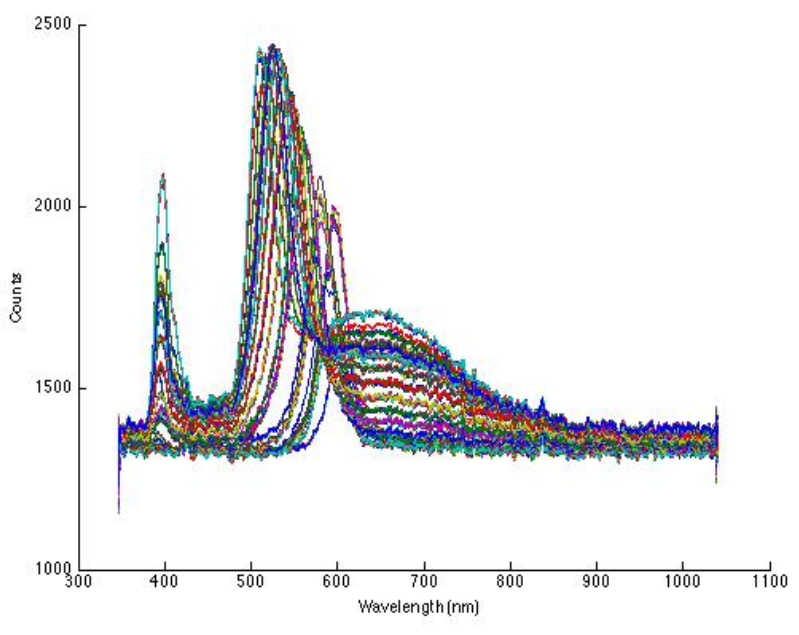

Trial 1 Stainless Steel 4_7_14

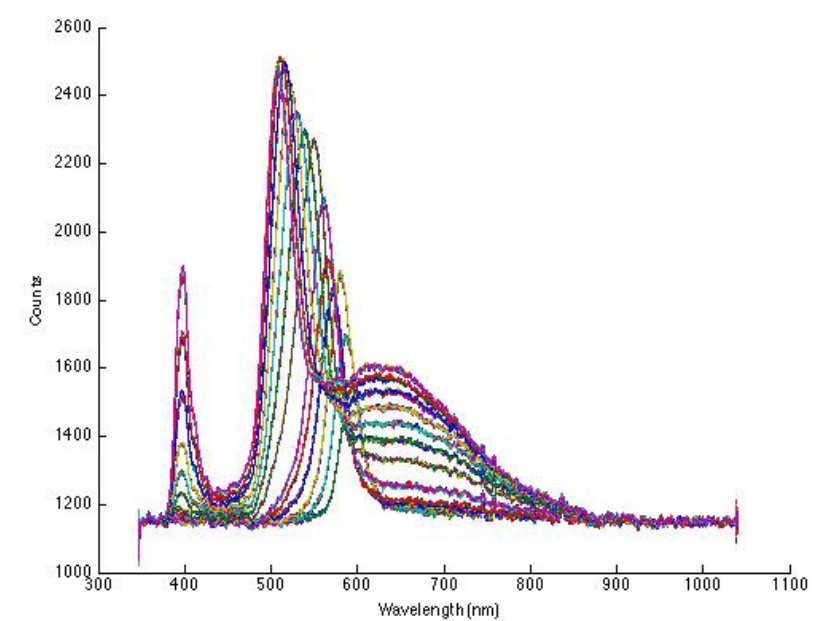

Trial 3 Stainless Steel 4_19_14

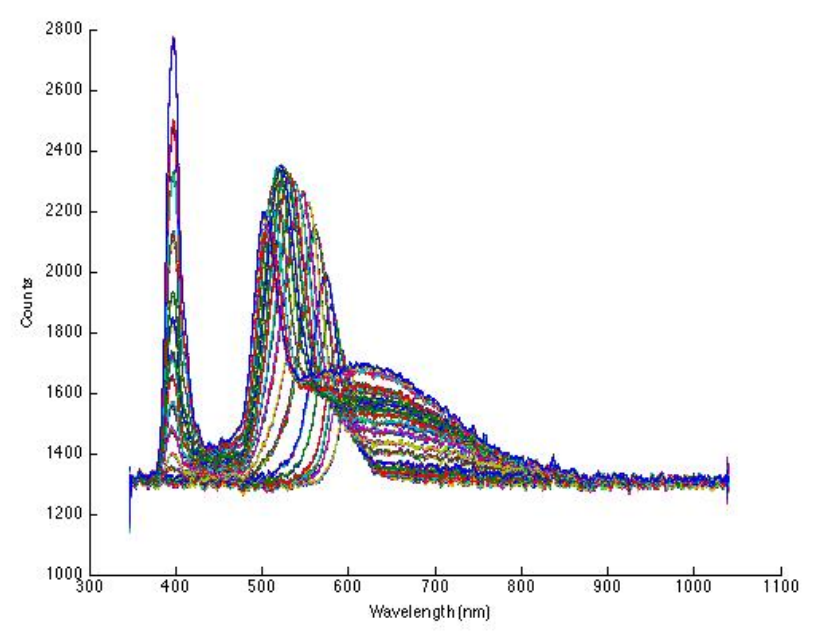

Trial 2 Stainless Steel 4_15_14

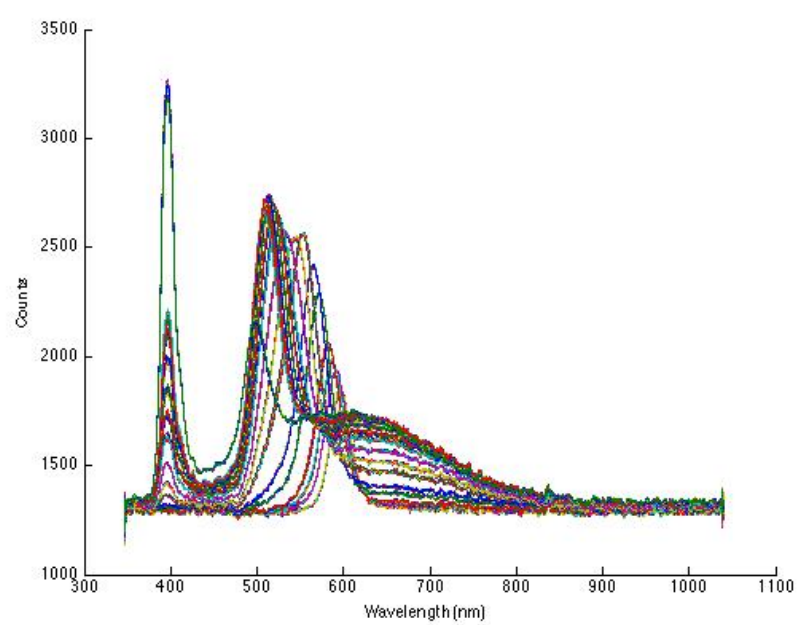

Trial 4 Stainless Steel 4_25_14 


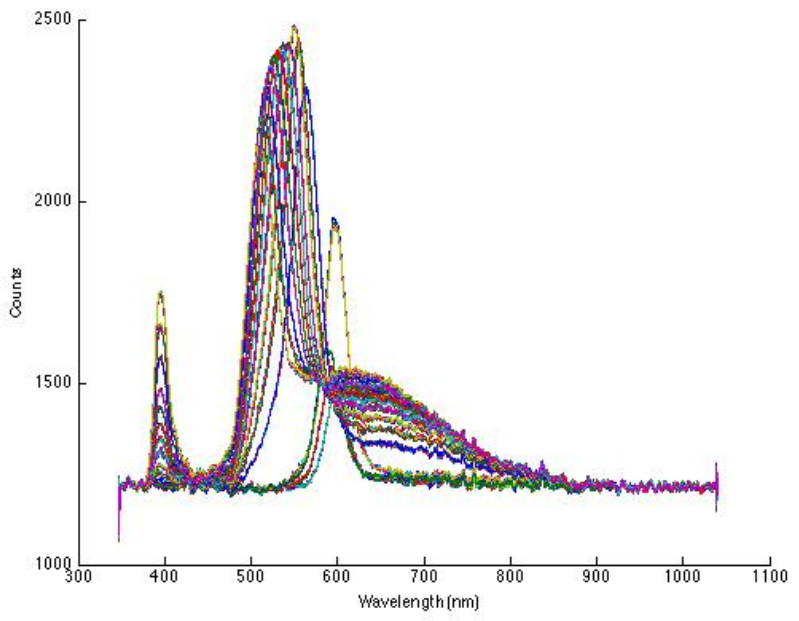

Trial 5 Stainless Steel 4_30_14

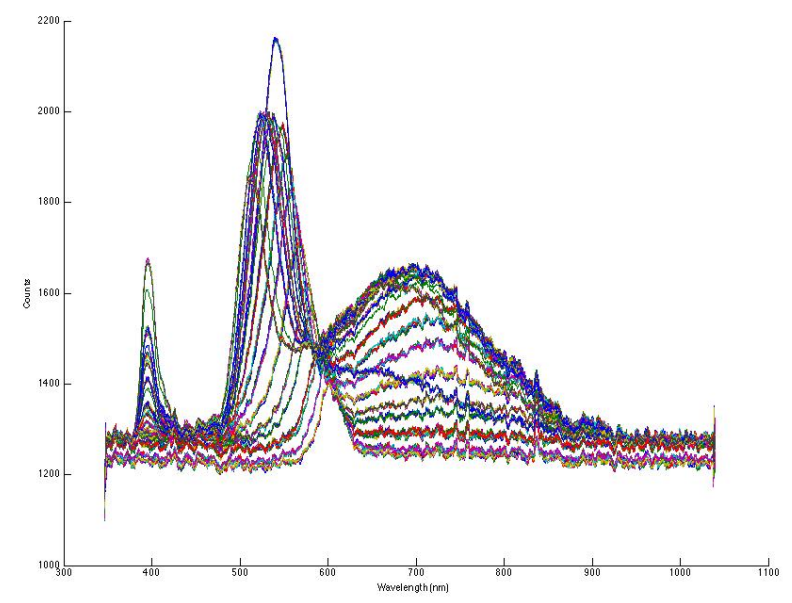

Trial 7 Stainless Steel 5_6_14

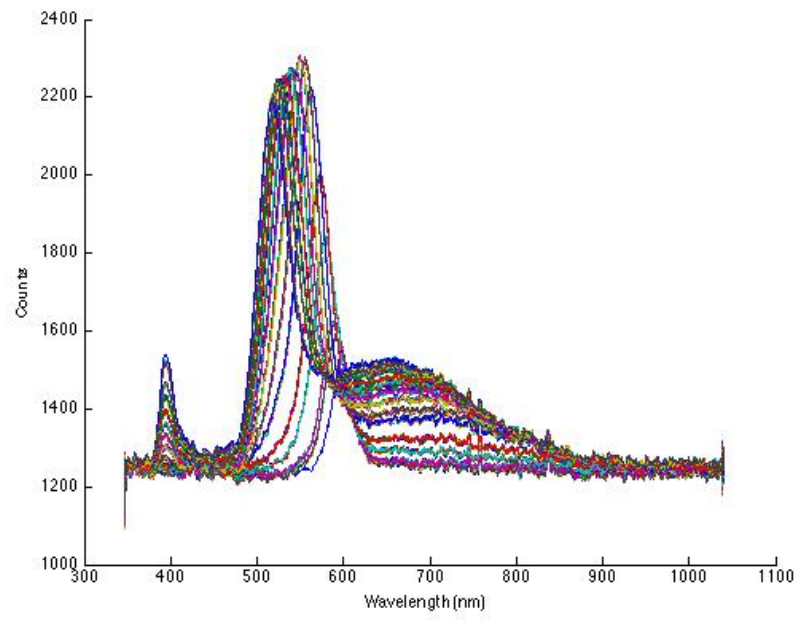

Trial 6 Stainless Steel 5_5_14

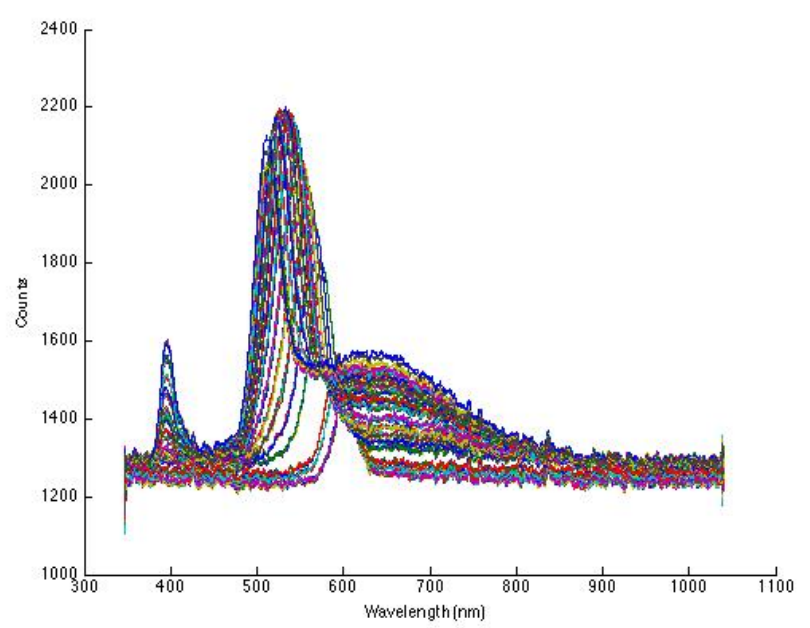

Trial 8 Stainless Steel 5_9_14 
Appendix E 316 Stainless Steel Reactor Three-Dimensional Topographic Spectral Profiles By Trial

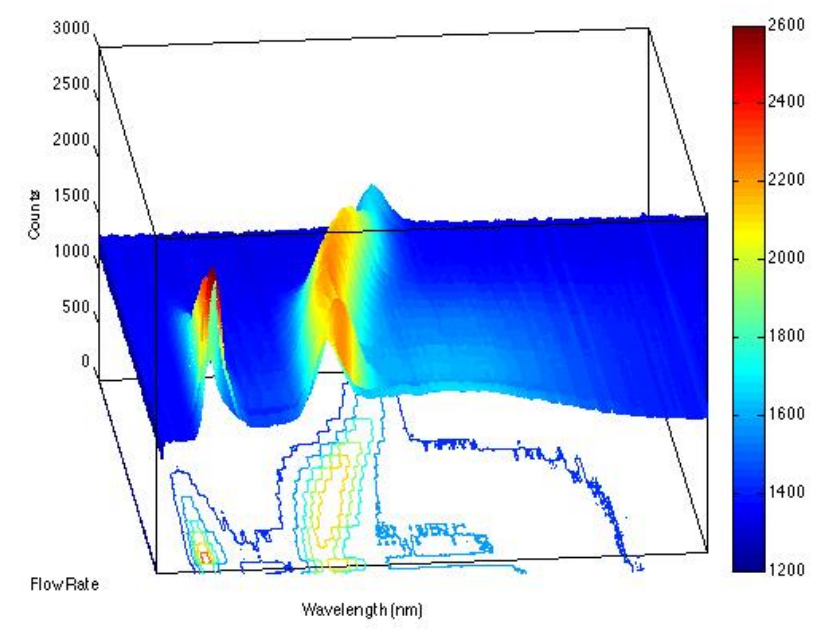

Trial 1 Stainless Steel 4_15_14

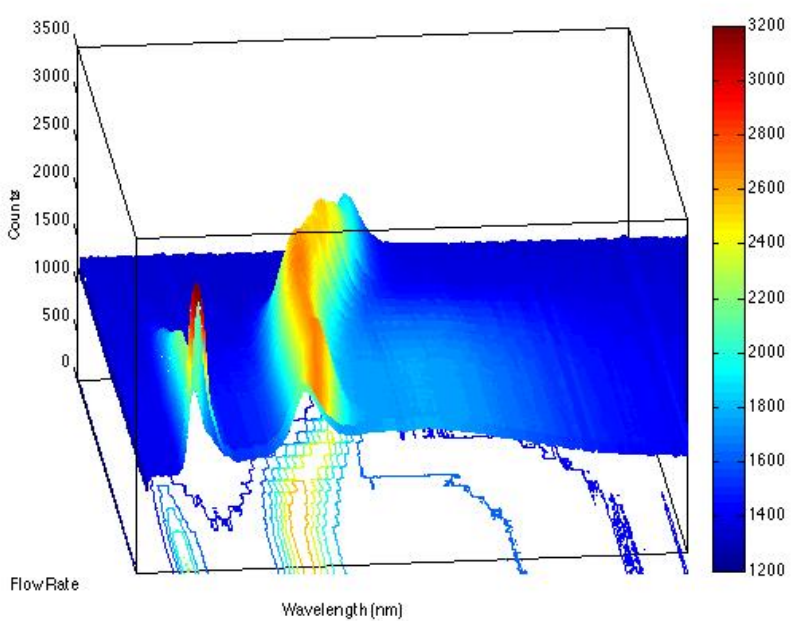

Trial 3 Stainless Steel 4_25_14

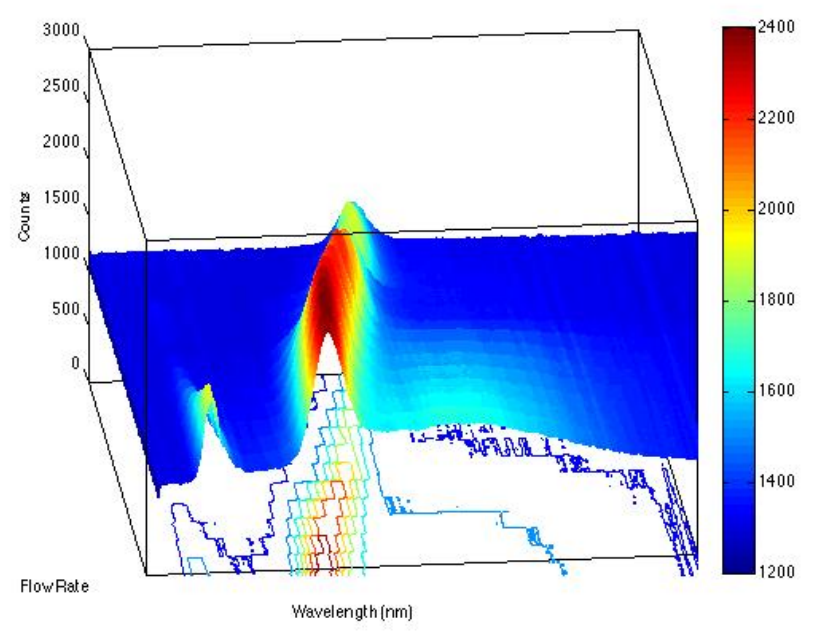

Trial 2 Stainless Steel 4_19_14

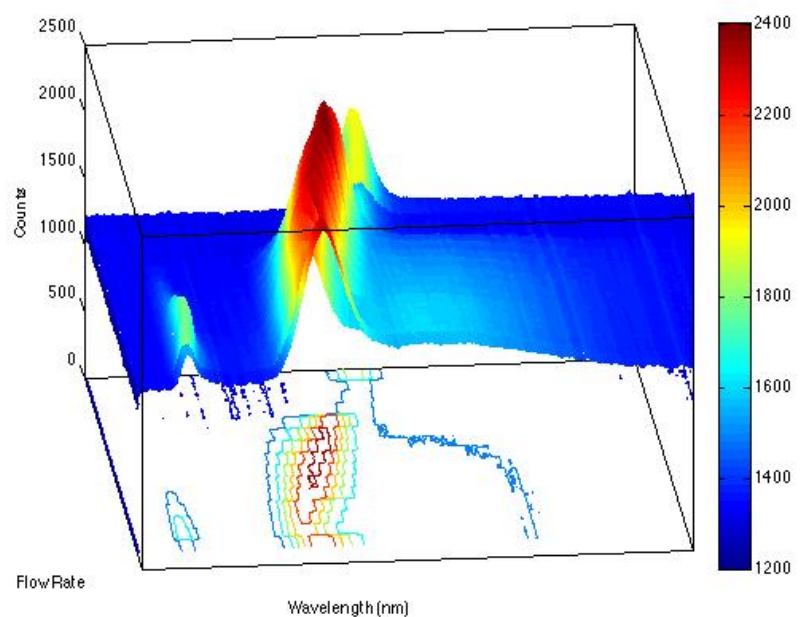

Trial 4 Stainless Steel 4_30_14 


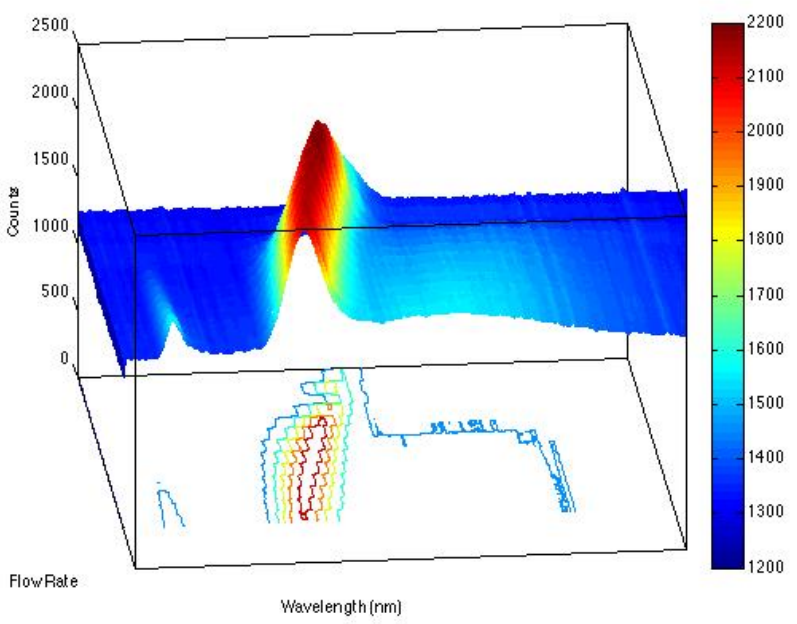

Trial 5 Stainless Steel 5_5_14

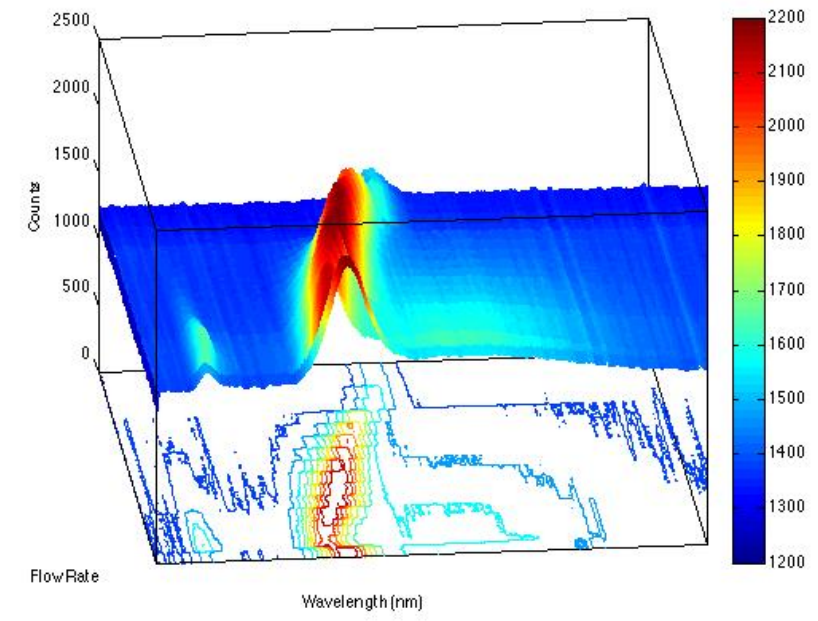

Trial 7 Stainless Steel 5_9_14

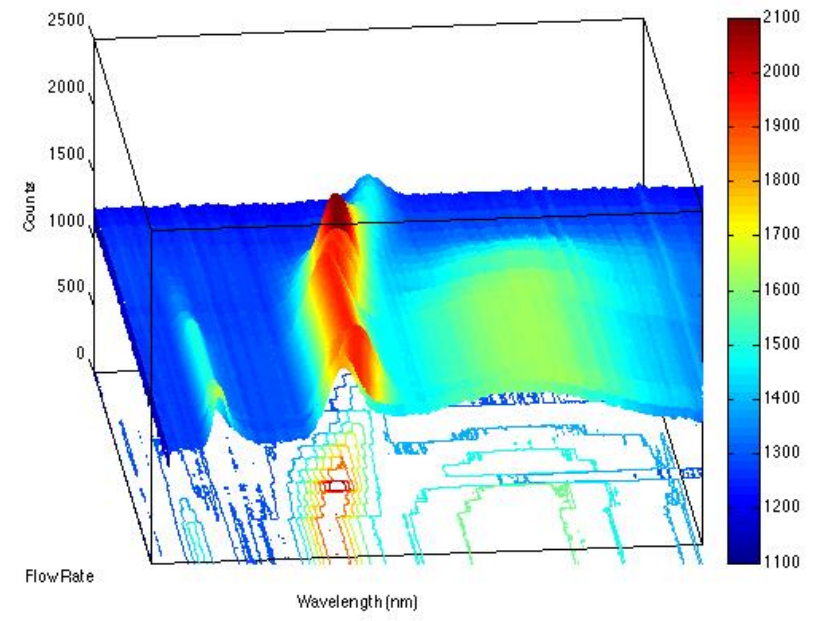

Trial 6 Stainless Steel 5_6_14 
Appendix F $\quad$ TEFLON $^{\circledR}$ Reactor Two-Dimensional Spectral Profiles By Trial

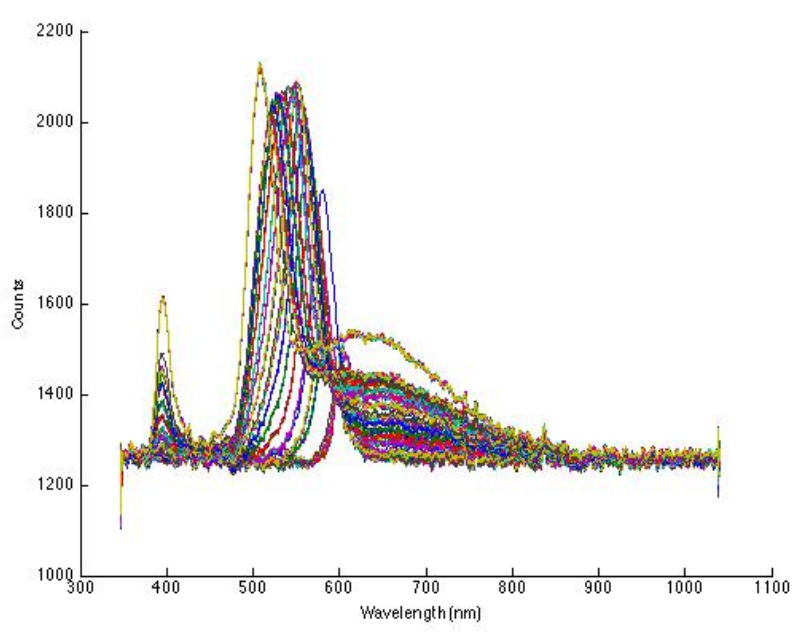

Trial 1 Teflon 5_28_14

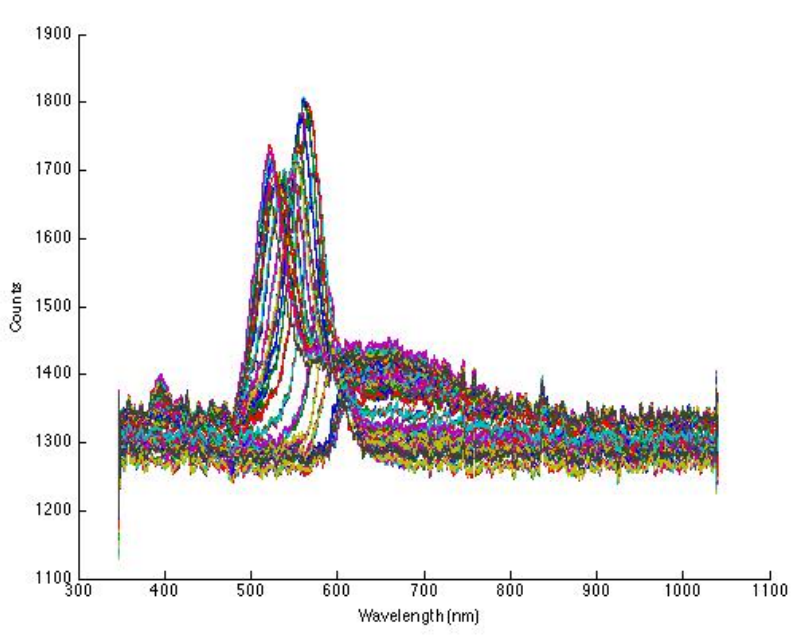

Trial 3 Teflon 6_23_14

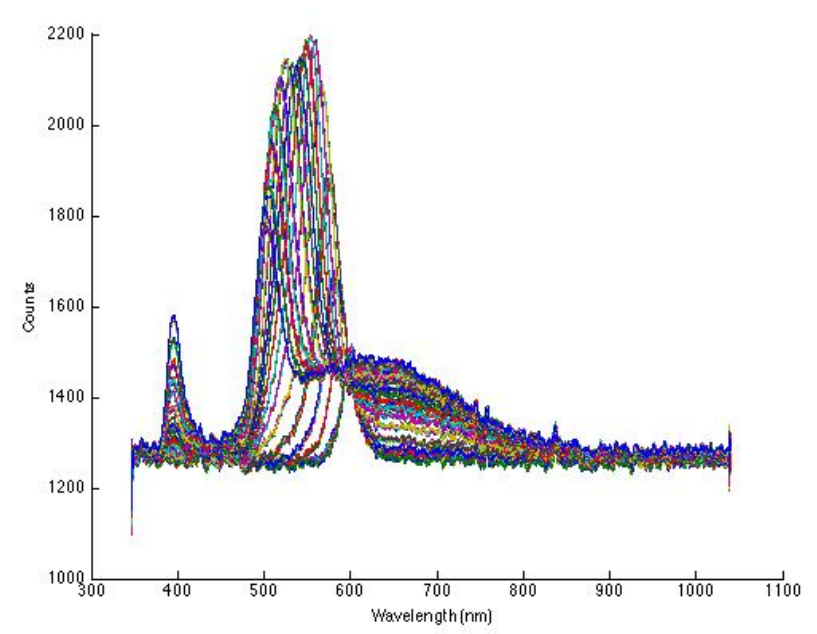

Trial 2 Teflon 6_21_14

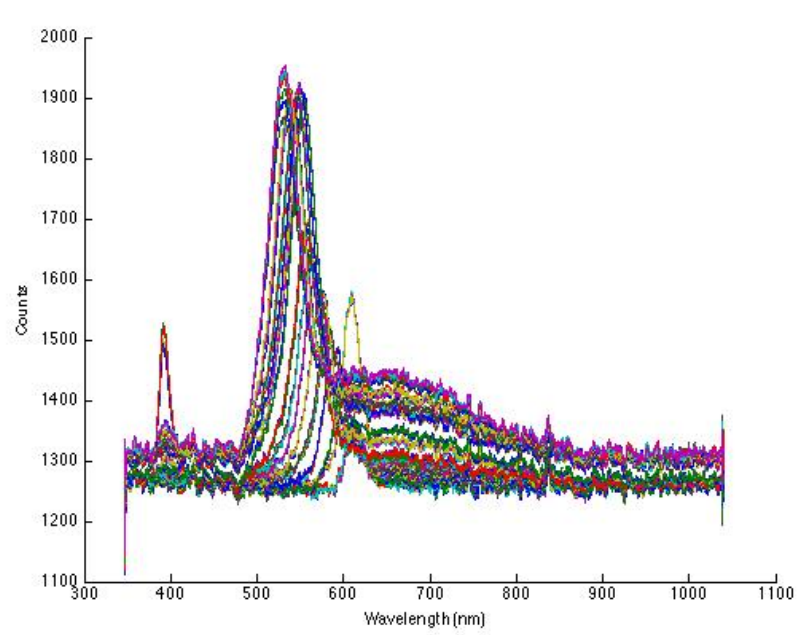

Trial 4 Teflon 6_24_14 
Appendix G TEFLON ${ }^{\circledR}$ Reactor Three-Dimensional Spectral Profiles By Trial

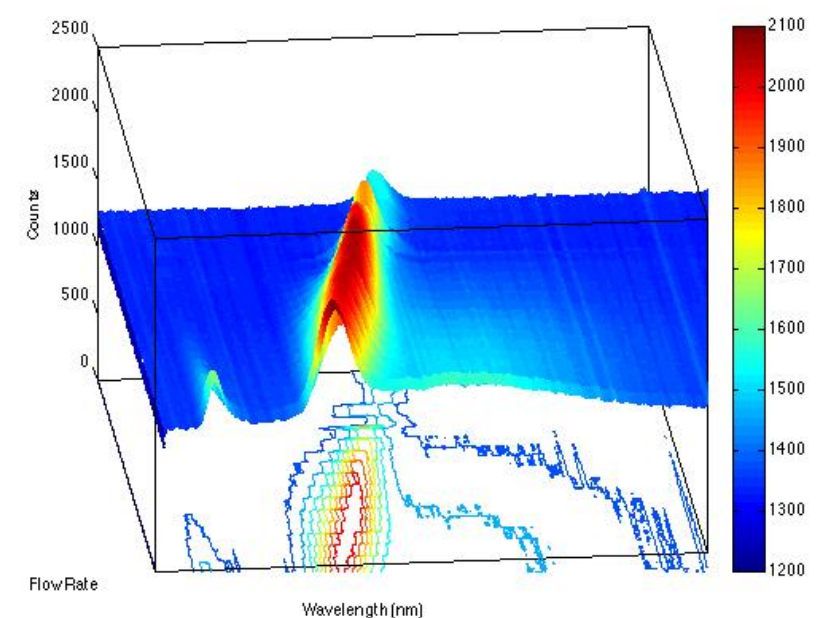

Trial 1 Teflon 5_28_14

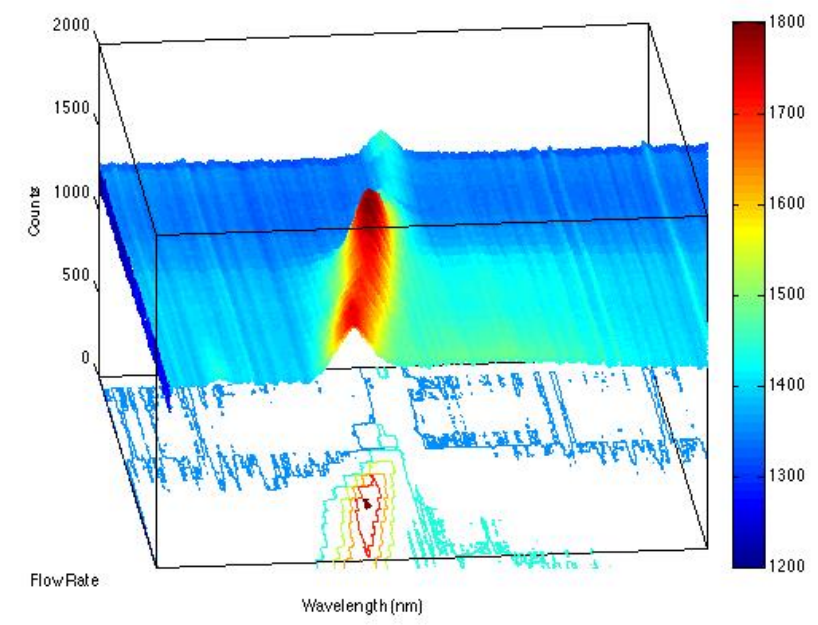

Trial 3 Teflon 6_23_14

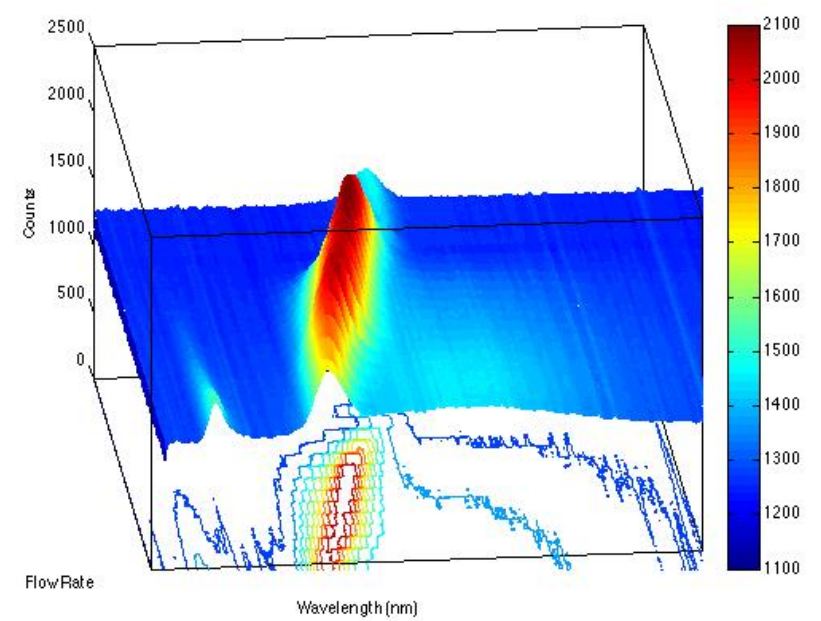

Trial 2 Teflon 6_21_14

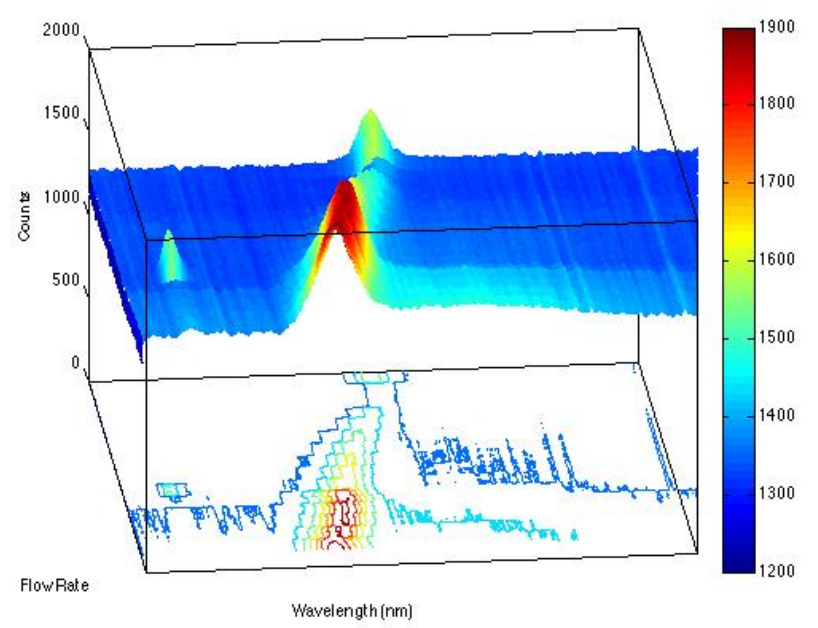

Trial 4 Teflon 6_24_14 


\section{Appendix H SEM Analysis Procedures}

\section{Sputtering Procedure}

Gold was sputtered on the SEM samples using a Denton IV desktop sputtering system. First the argon canister valve was opened then the samples were loaded in the vacuum chamber and covered by the shutter. The lid was replaced and the roughing pump was turned on pulling a vacuum at $50 \mathrm{mT}$ Trr. At this point the gas valve was opened flooding the chamber with argon gas. Pressure was allowed to equalize at $100 \mathrm{mTorr}$. The Manual sputter option was selected with a set point of 30 . After 90 seconds the sputter power and roughing pump were turned off and the samples were removed from the vacuum chamber. The samples were then transferred to the Jeol SEM for analysis.

\section{SEM Procedure}

The two tubing samples attached to a $12 \mathrm{~mm}$ stud were locked into the aluminum chuck using a setscrew and a piece of conductive copper tape was attached, bridging the sample surface and the outer edge of the aluminum chuck. The sample height (3mm) was measured from the top of the aluminum chuck. The sample stage was set to a height of $13 \mathrm{~mm}$ to obtain a working distance of $10 \mathrm{~mm}$. The chamber was then vented after turning off the pressure gauge. The aluminum chuck was loaded into the SEM and the door was closed carefully ensuring the sample cleared the column. The chamber was evacuated for 15 minutes to ensure a high mean free path for the electron beam to increase resolution. For the stainless steel sample the beam voltage was set to $20 \mathrm{kV}$ with a spot size of 75 . The beam voltage was set to $2.5 \mathrm{kV}$ to minimize charging on the nonconductive 
TEFLON $^{\circledR}$ tubing and a spot size of 75 was selected. For both samples three images were taken at $95 \mathrm{X}, 450 \mathrm{X}$ and $2000 \mathrm{X}$.

\section{Appendix I Gwyddion Surface Roughness Test Parameters}

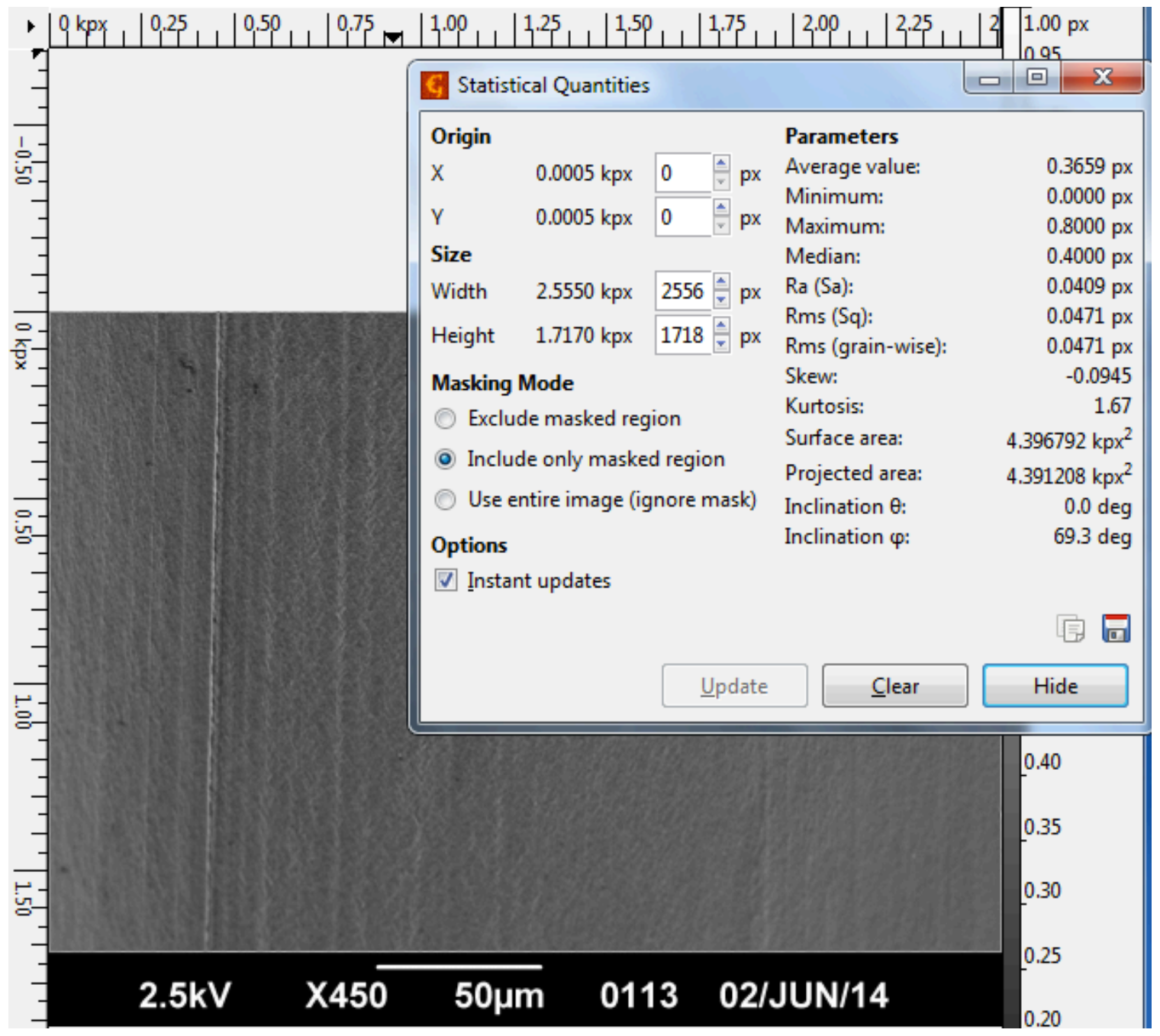

Surface roughness test using Gwyddion. Note the sample size remained the same for all images. RMS $(\mathrm{Sq})$ is the surface roughness parameter that was compared. 


\section{Appendix J AFM Experimental Methods}

Octadecene exhibits extremely low evaporation rates and leaves behind a film making it a less than ideal solvent to transfer quantum dots to a substrate. To remedy this the quantum dots were extracted from the octadecene and transferred to a toluene suspension. The procedure for this is explained below.

\section{Precipitation of QDs}

Octadecene suspended QDs were precipitated using 200 proof ethanol (EtOH). EtOH with a density of $0.789 \mathrm{~g} / \mathrm{mL}$ sinks below octadecene $0.77 \mathrm{~g} / \mathrm{mL}$ when mixed in solution. The solid Cd-Se quantum dots have a higher density than both solvents. When centrifuged, the QDs were pulled by centrifugal force from the octadecene solution into the denser ETOH solution and come to rest in a pellet at the bottom of the sample container. The procedure used in this separation was taken from previous thesis.

\section{Centrifuge Procedure}

The centrifuge used in this procedure had a six test tube capacity and required a symmetric distribution of both position and weight to minimize vibration and damage to the equipment. The initial QD samples were split into two equal volumes and placed in two test tubes directly opposite from each other. Two parts by volume EtOH was added to each one-part QD solution (1 ml octadecene QD solution mixed with $2 \mathrm{ml} \mathrm{EtOH})$. The octadecene and $\mathrm{EtOH}$ mixture initially resembled an oil and water mixture where the oil forms spherical droplets. The mixtures were shaken to increase the surface area of the phase intersections to allow for more efficient transfer of Cd-Se dots to ETOH. The test 
tubes were subjected to a centrifuge cycle at $4000 \mathrm{rpm}$ for 10 minutes. Following this cycle the clear supernatant was removed leaving the colored octadecene QD solution and a pellet and more EtOH was added. The centrifuge cycle, removal of supernatant and addition of EtOH was repeated until a viscous pellet of QDs had formed at the base of the test tube. The majority of EtOH and octadecene was then removed and the remaining pellet was left in a fume hood for $48 \mathrm{hrs}$ to allow the remaining EtOH to evaporate.

\section{Re-Suspension}

Toluene was selected to re-suspend the QDs because it evaporates quickly at room temperature and possesses strong nonpolar characteristics that prevent coalescence of QDs. 3ml toluene was added to the containers and the containers were sonicated in an ultrasonic bath to re-suspend the ODs.

\section{Dilution}

Initially the toluene solution was used as is, however, it appeared that significant coalescence occurred after dispensing the solution on the substrate. To remedy this a small sample of the remaining solution was diluted between 5000 and 10000 times with toluene to prevent coalescence. 


\section{Sample Substrate}

A smooth substrate was required to measure quantum dots between $2 \mathrm{~nm}$ and $10 \mathrm{~nm}$ in height. Any surface roughness above the nanometer scale would obscure measurements. The substrate chosen was a 1-inch (111) polished silicon wafer with surface roughness of less than $1 \mathrm{~nm}$ RMS. The diluted quantum dots were deposited on this wafer with a single spray from a hobby airbrush. The airbrush acts as an atomizer depositing microdroplets of QD solution on the sample surface. The solvent then evaporates and the particles adhere to the surface. The wafer was cleaned both initially and after each scan. The sample surface was cleaned by immersion in $2500 \mathrm{ml}$ Piranha (9:1 ratio $98 \% \mathrm{H} 2 \mathrm{So} 4$ to $30 \% \mathrm{H} 2 \mathrm{O} 2$ ) at $70^{\circ} \mathrm{C}$ for 10 minutes, rinsed in deionized water by dipping 4 times and

dipping in a buffered oxide etchant. For each scan new quantum dots were applied via the atomizer.

\section{AFM Parameters}

Scans were performed using an Asylum AFM. The AFM was loaded with an Olympus AC 160 tip in non-contact mode. A scan area of $5 \mu \mathrm{m}$ was analyzed. A scan rate of $1 \mathrm{~Hz}$ was used with an adaptive PI feedback loop. Before scanning a baseline surface roughness of 46pm RMS height deviation was established for a 1-inch (111) polished silicon wafer. This low surface roughness reduces noise allowing for more accurate measurements of QDs. 